TOWSON UNIVERSITY

OFFICE OF GRADUATE STUDIES

\title{
ACCESSIBLE COLLABORATIVE WRITING FOR \\ PERSONS WHO ARE BLIND
}

\author{
By \\ John G. Schoeberlein \\ A Dissertation \\ Presented to the faculty of \\ Towson University \\ In partial fulfillment \\ Of the requirements for the degree \\ Doctor of Science in Information Technology \\ Department of Computer and Information Sciences
}

Towson University

Towson, Maryland 21252

December 2013 


\section{TOWSON UNIVERSTTY \\ OFFICE OF GRADUATESTUDIES}

\section{DISSERTATION APPROVAL PAGE}

This is to eertity that the dissertation prepared by John Sehwetwritein entitted, "Accessible Collaborative Writing for persons who are lbind," has becn approved ty the disserbtion commitce as satistitctory conplcting the dissertation requircments for the

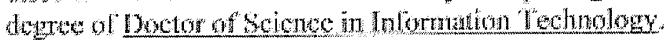

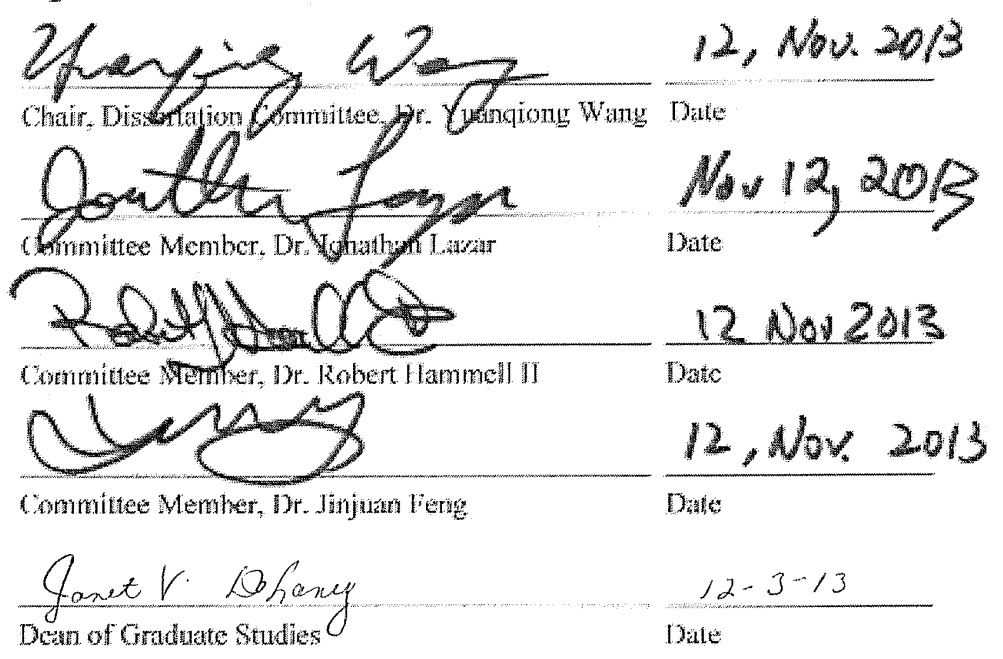


(C) 2013 by John G. Schoeberlein All Rights Reserved 


\section{ACKNOWLEDGEMENTS}

I would like to acknowledge Drs. Jinjuan Feng, Robert Hammell II, Jonathan

Lazar, and Yuanqiong Wang for their guidance, recommendations and insights, and the Members of the National Federation of the Blind for their support and participation in this research. I would like to thank my wife, Jackie Lynn Schoeberlein, for her continued support and understanding while I pursued this adventure. 


\section{ABSTRACT \\ ACCESSIBLE COLLABORATIVE WRITING FOR PERSONS WHO ARE BLIND}

\section{JOHN G. SCHOEBERLEIN}

In order to establish the current state of the accessibility and the usability of collaborative technologies, a literature review, several focus group studies and field studies were conducted. The collaborative technologies examined were email, Chat, group calendars, and collaborative writing. As a result of these examinations, collaborative technologies were determined to be inaccessible and unusable, often leading to end-user frustration and discontinued use of the technology. The participants of the focus group studies and field studies reported that collaborative writing was necessary on a daily basis, but was inaccessible and unusable.

Therefore, as a consequence of the accessibility and usability issues found with collaborative writing applications, a proposed accessible and usable Microsoft Word Add-In prototype was developed for Microsoft Word. The interface design included such features as a context-sensitive menu and hot-keys for easy access and use. Utilizing an iterative design approach, the prototype was examined in two rounds of usability study 
with participants' suggested improvements included in-between each round. The resulting prototype was considered easy to learn and use, clear and understandable, improved the participants' performance, and would be utilized by the participants on a regular basis to do similar tasks. 


\section{TABLE OF CONTENTS}

ACKNOWLEDGEMENTS ......................................................................................... iv

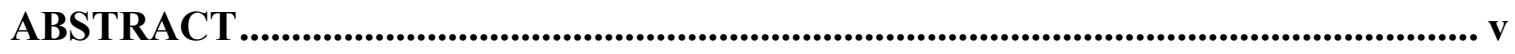

TABLE OF CONTENTS ...................................................................................... vii

LIST OF TABLES .......................................................................................................... xiii

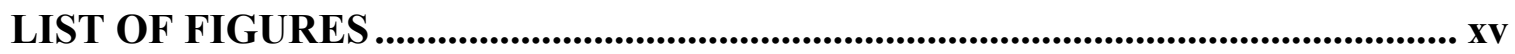

1. INTRODUCTION ........................................................................................................ 1

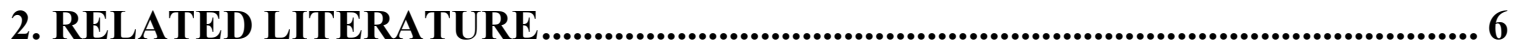

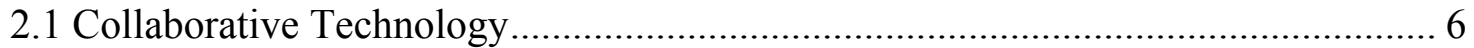

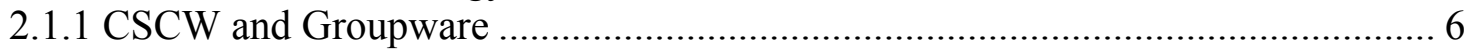

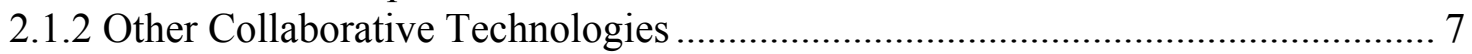

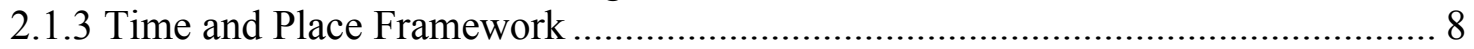

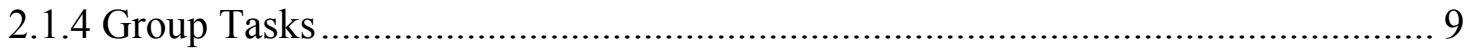

2.1.5 The Impact of Collaborative Technologies ................................................... 12

2.1.6 The Uniqueness of Collaborative Technologies ............................................... 13

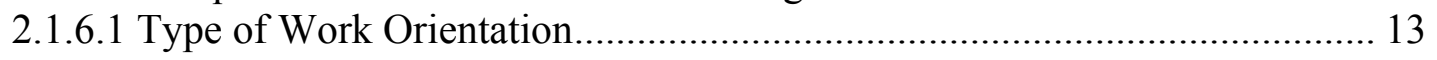

2.1.6.2 Number of Users Supported .................................................................. 14

2.1.6.3 Network Support................................................................................ 15

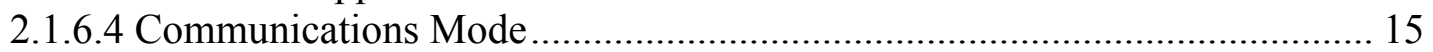

2.1.6.5 Floor Control, Concurrency Control........................................................ 15

2.2. Accessibility and Usability ......................................................................... 16

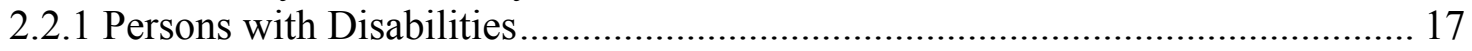

2.2.2. Adaptive Technologies Available to Persons with Disabilities ........................... 18

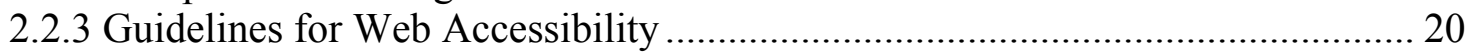

2.2.4 Guidelines for Windows Applications............................................................... 22

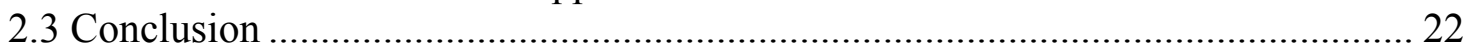

3. TECHNOLOGY EVALUATION AND APPLICATION COMPARISION ........ 23

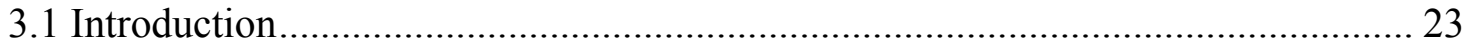

3.2 Collaborative Technology Accessibility Evaluation Framework ........................... 23

3.3 Applications for Evaluation Selection ................................................................. 26

3.4 Automatic Accessibility Evaluations................................................................ 28

3.4.1 Web-based Applications' Evaluation Criteria............................................... 28

3.4.2 Web-based Applications' Evaluation Procedure …………………………....... 30

3.4.3 Web-based Collaborative Technology Evaluations........................................... 30

3.4.3.1 Fujitsu's Web Accessibility Inspector - WCAG 1.0 …................................ 30

3.4.3.2 Adaptive Technology Resource Center (ATRC) Web Accessibility Checker

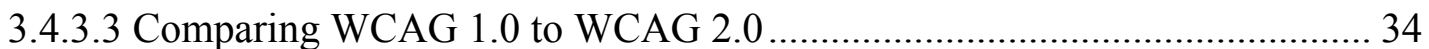

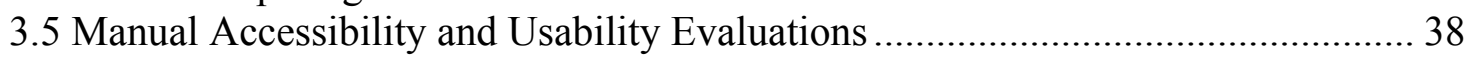

3.5.1 Windows, Console and Web Applications' Evaluation Criteria ..................... 39 
3.5.2 Windows, Console and Web Applications' Evaluation Procedure ................. 40

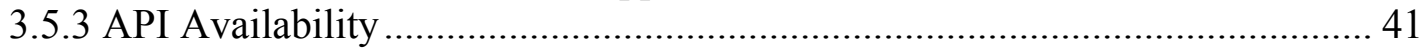

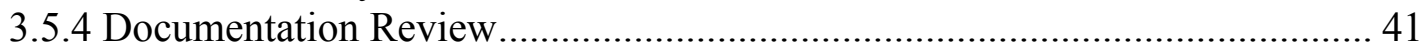

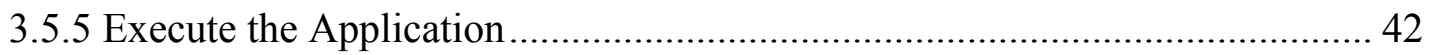

3.5.5.1 Microsoft Outlook Evaluation ..................................................................... 43

3.5.5.2 America Online Instant Messenger (AIM $\left.{ }^{\circledR}\right)$ Evaluation ................................ 46

3.5.5.3 Twitter Evaluation .................................................................................... 47

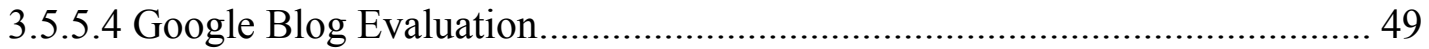

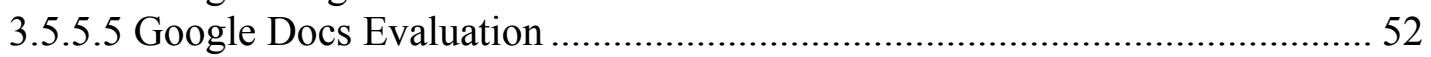

3.5.5.6 Group Systems' Think Tank Evaluation .................................................... 53

3.5.5.7 Microsoft SharePoint Evaluation............................................................... 56

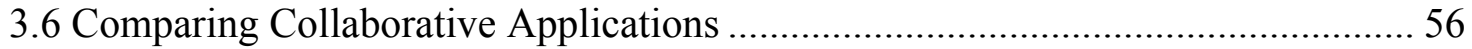

3.6.1 Accessible Collaborative Interface Prototypes ............................................... 56

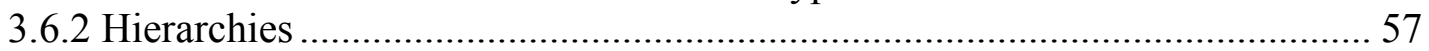

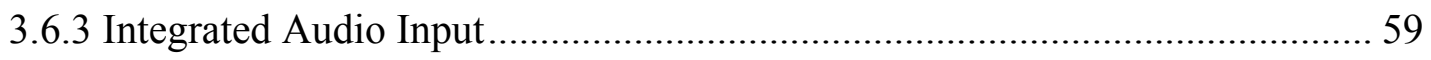

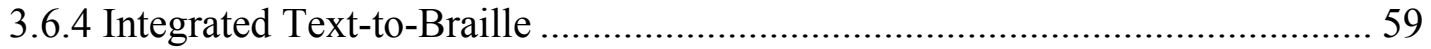

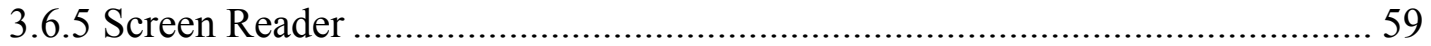

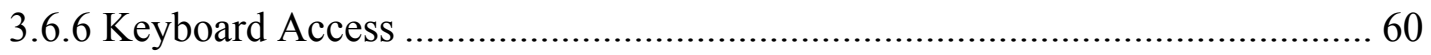

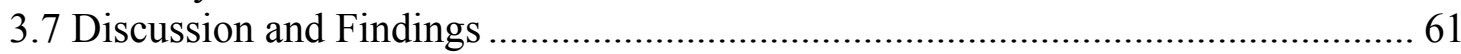

3.8 Potential Approaches for Developing Accessible Prototypes ................................ 63

3.8.1 Application Programming Interface (API) for Prototype Development .......... 63

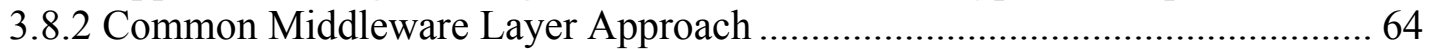

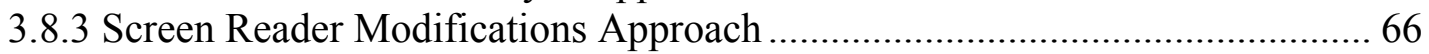

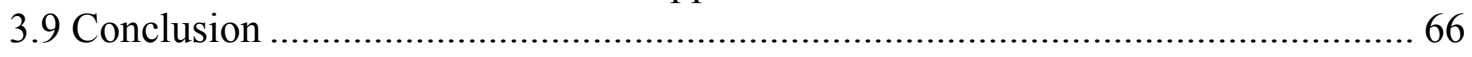

4. INITIAL FIELD VISITS TO THE NATIONAL FEDERATION OF THE BLIND

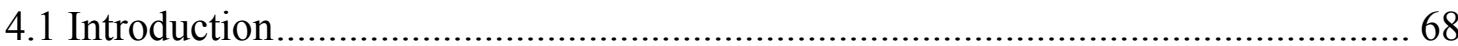

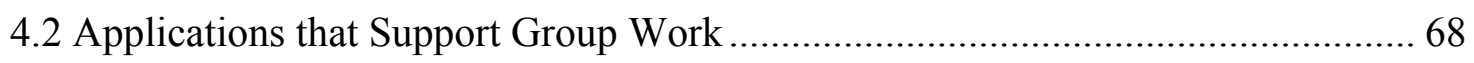

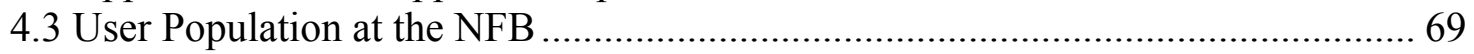

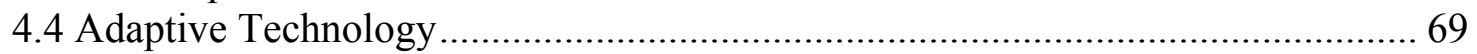

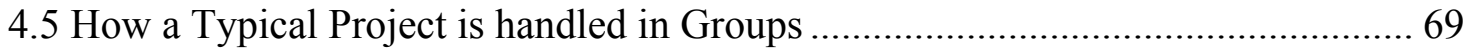

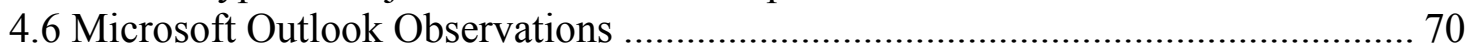

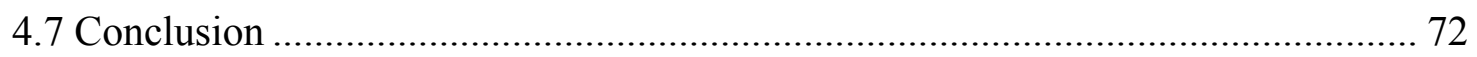

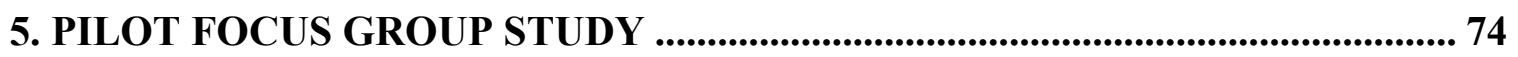

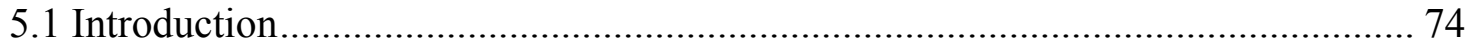

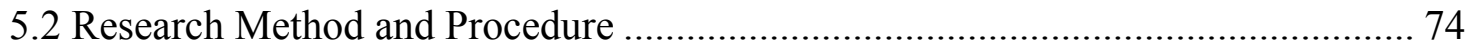

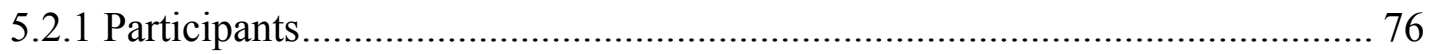

5.3 Data Analysis Method - The Grounded Theory Criteria for Evaluation ............... 77

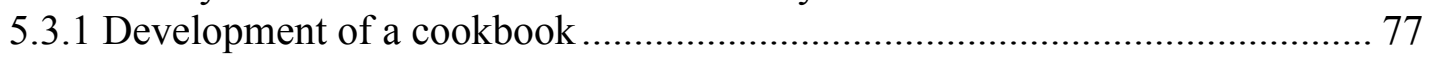

5.3.2 Establishing Inter-coder Reliability ………………...................................... 78

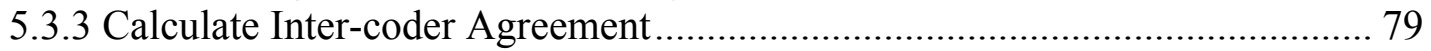

5.4 Results from the Pilot Focus Group Study ………….............................................. 80

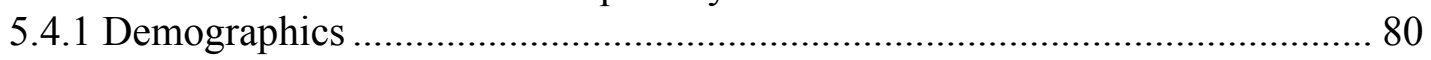

5.4.2 Content Analysis of the Focus Group Study Recording ................................. 81

5.4.2.1 Participants Identification of Collaborative Software Utilized..................... 83

5.4.2.2 The Tasks/Steps Necessary to Complete a Group Project.............................. 84 
5.4.2.3 Accessibility and Usability Issues Experienced ........................................... 84

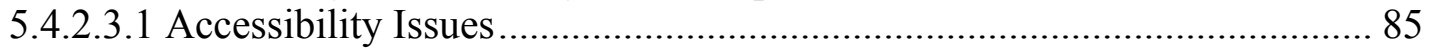

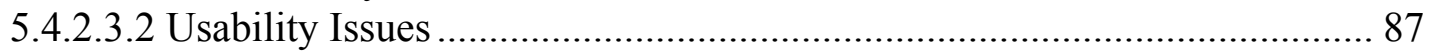

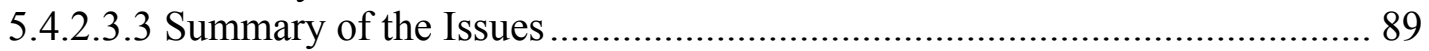

5.4.2.4 Collaborative Applications Utilized, but Discontinued ................................. 89

5.4.2.5 Other Tools Utilized to Support Group Work .............................................. 90

5.4.2.6 Accessibility Design Considerations ............................................................ 91

5.4.2.7 Suggestions on Accessibility Documentation and Support .......................... 91

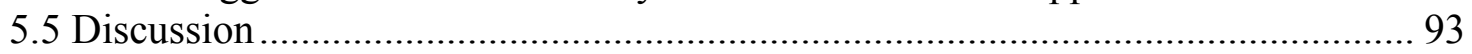

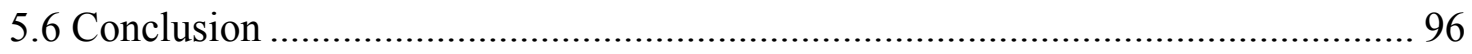

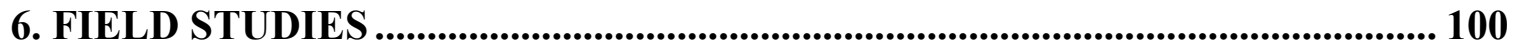

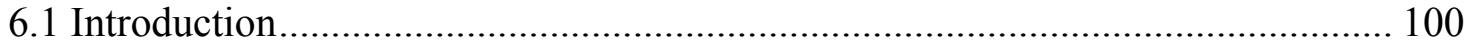

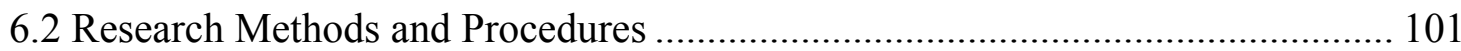

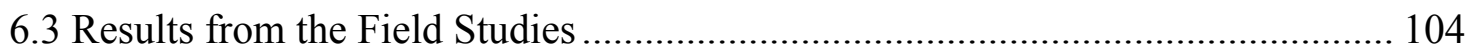

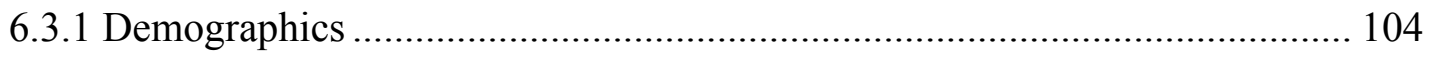

6.3.2 Content Analysis of the Field Studies' Recordings ....................................... 104

6.3.2.1 Participants identification of collaborative software utilized ...................... 106

6.3.2.2 The Tasks/Steps Necessary to Complete a Group Project........................... 108

6.3.2.3 Accessibility and Usability Issues Observed/Discussed.............................. 109

6.3.2.3.1 Microsoft Outlook Issues...................................................................... 110

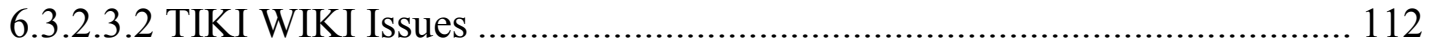

6.3.2.3.3 Screen Reader Interactions' Issues ........................................................ 112

6.3.2.4 Group interaction techniques ................................................................. 114

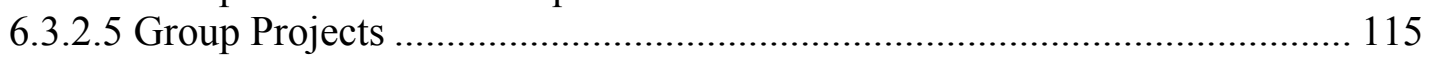

6.3.2.6 Accessibility design considerations ........................................................ 115

6.3.2.7 Suggestions on accessibility documentation and support............................ 118

6.3.2.8 Collaborative Applications' Benefits..................................................... 119

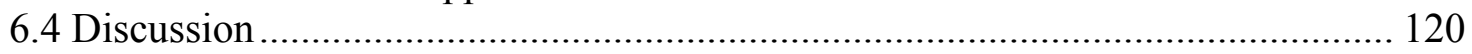

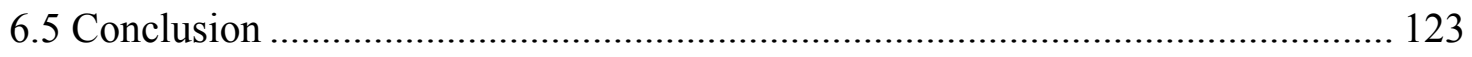

7. ADDITIONAL FOCUS GROUP STUDIES ......................................................... 127

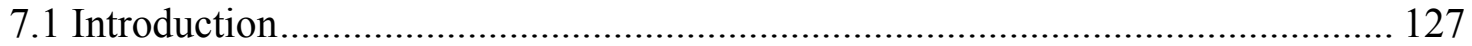

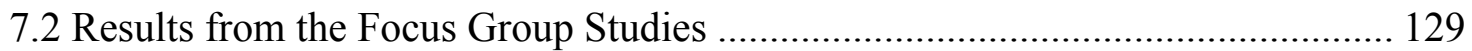

7.2.1 Demographics and Experience ................................................................ 129

7.2.2 Data Analysis of the Focus Group Study Recording ..................................... 131

7.2.2.1 Participants identification of collaborative software utilized ...................... 131

7.2.2.2 The Tasks/Steps Necessary to Complete a Group Project........................... 133

7.2.2.3 Accessibility and usability issues experienced ........................................... 134

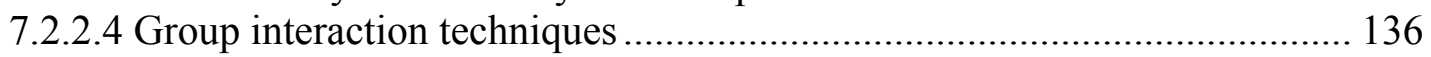

7.2.2.5 Accessibility design considerations ........................................................... 137

7.2.2.6 Suggestions on accessibility documentation and support........................... 137

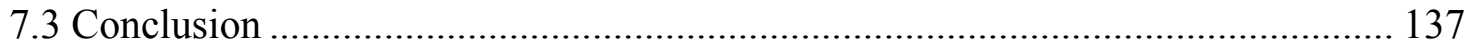

8. IDENTIFYING RESEARCH OPPORTUNITIES ................................................ 141

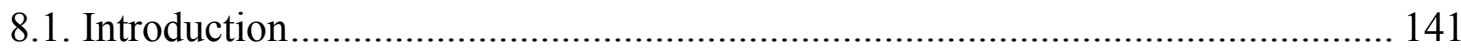

8.2 Collaborative Writing Research....................................................................... 144

8.3 Manual Examination of Google Docs and Microsoft Word.................................. 147 


\section{COLLABORATIVE WRITING RESEARCH DESIGN AND METHODOLOGY}

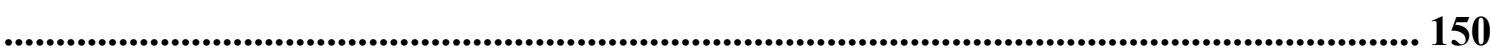

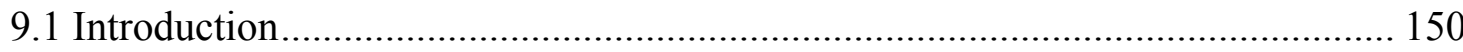

9.2 Research Method .................................................................................... 152

9.2.1 Phase One - Baseline Usability Studies .................................................. 152

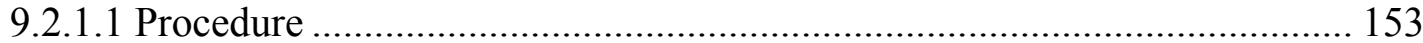

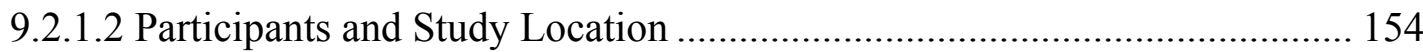

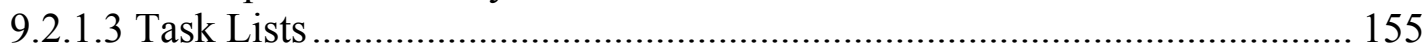

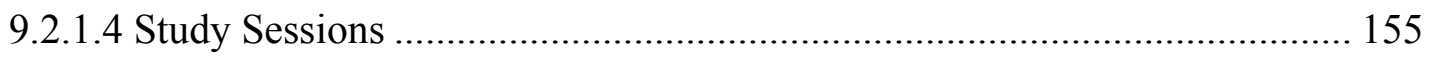

9.2.2 Phase Two - Development of an accessible and usable collaborative writing

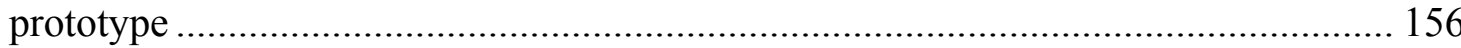

9.2.3 Phase Three - Pilot Usability Study ........................................................ 157

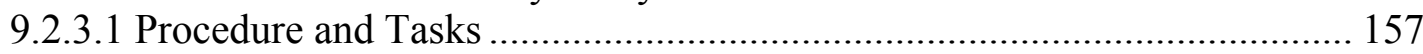

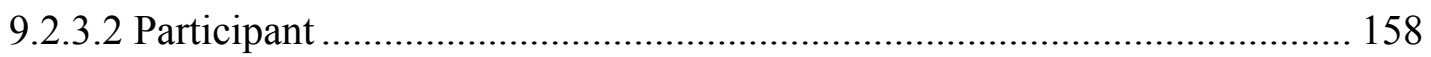

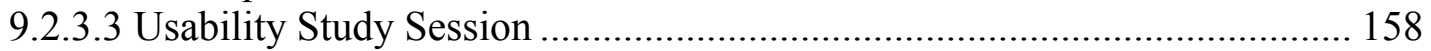

9.2.4 Phase Four - Enhancement of the collaborative writing prototype................. 158

9.2.5 Phase Five - Final Usability Study ............................................................. 159

9.3 Data Collection Methods ................................................................................. 159

9.4 Qualitative Data Analysis Method - The Grounded Theory .............................. 160

9.5 Quantitative Data Analysis Method.............................................................. 160

10. APPLICATION DESIGN AND USABILITY EVALUATIONS ..................... 161

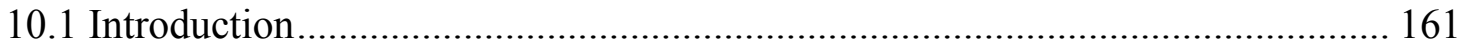

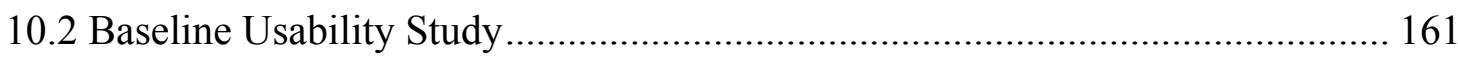

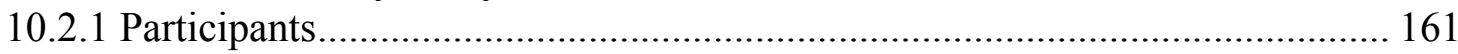

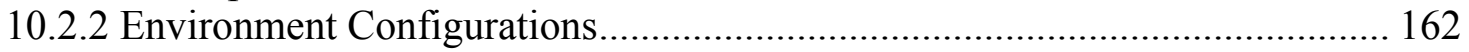

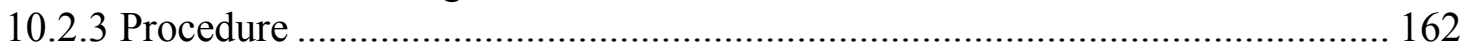

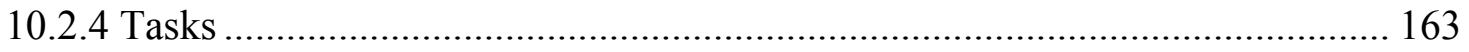

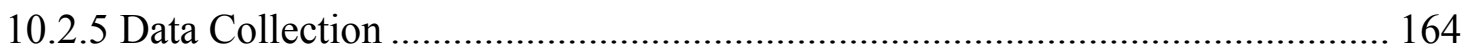

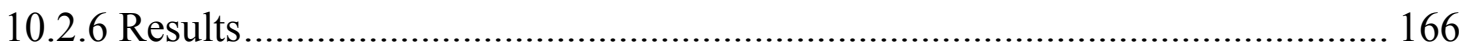

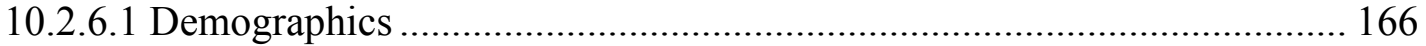

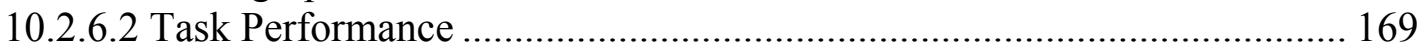

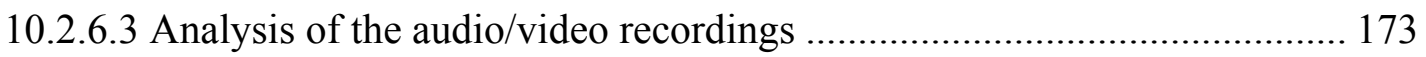

10.2.6.3.1 Visually able participants............................................................... 175

10.2.6.3.2 Participants Who Are Blind ............................................................. 176

10.2.6.4 The Collaborative Writing Experience .................................................. 177

10.2.6.5 Perception of the Collaborative Writing Experiences ............................. 184

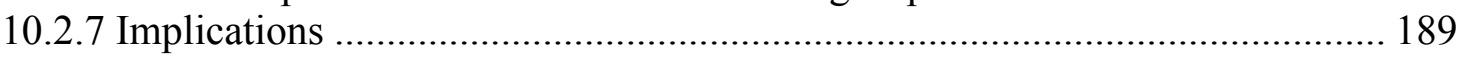

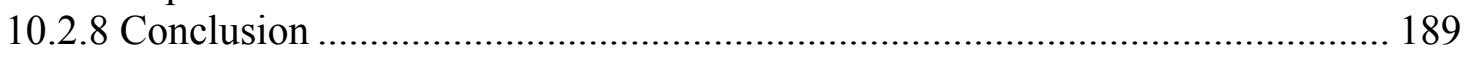

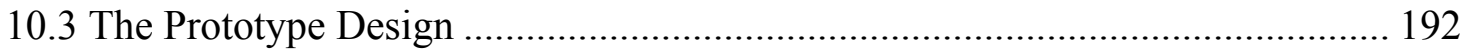

10.3.1 Microsoft Word Application Add-In ....................................................... 192

10.3.2 Microsoft Word Add-In Features Designed/Implemented ........................... 193

10.3.2.1 Comprehensive View.......................................................................... 194

10.3.2.2 Present the context (paragraph or sentence) of each comment and revision

10.3.2.3 Identify each Revision as an Addition, a Deletion, or a Change .............. 195 
10.3.2.4 Present each comment separately ........................................................... 195

10.3.2.5 Select/highlight each revision/comment in a series................................. 195

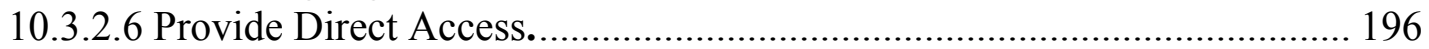

10.3.2.7 Revision View versus Final View. ......................................................... 197

10.3.2.8 JAWS and Built-In Audio.................................................................... 198

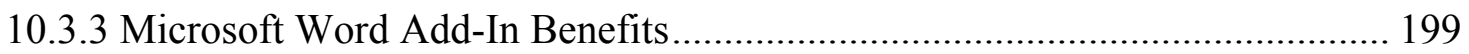

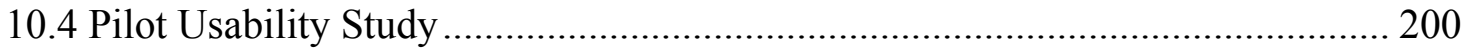

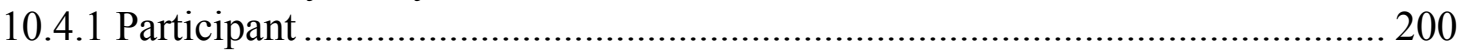

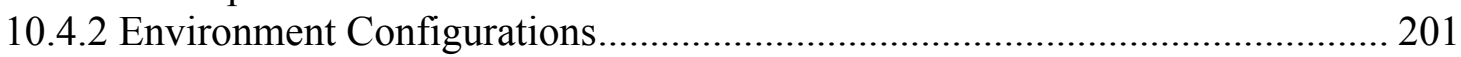

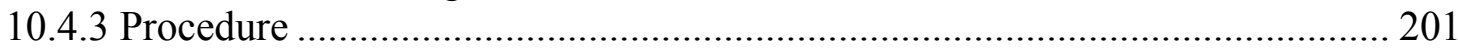

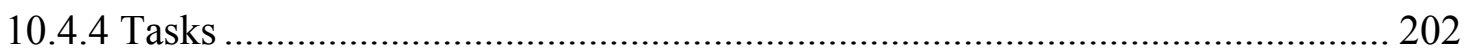

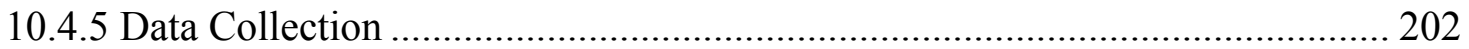

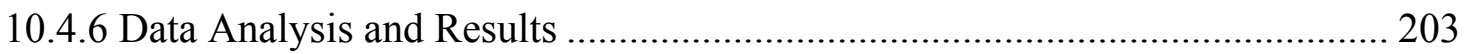

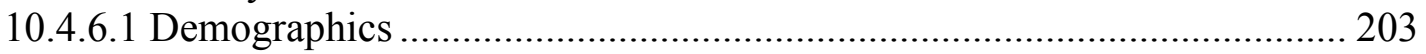

10.4.6.2 Task Completion Percent and Mean ........................................................ 203

10.4.6.3 Analysis of the audio/video recordings .................................................. 204

10.4.6.4 The Collaborative Writing Experience ………....................................... 205

10.4.6.5 Perceptions/Feedback Collected on Collaborative Writing Experiences . 206

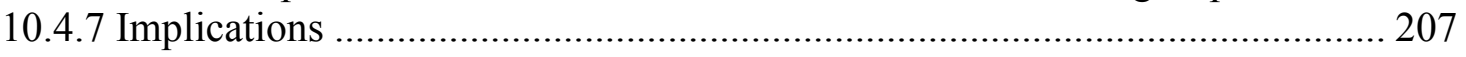

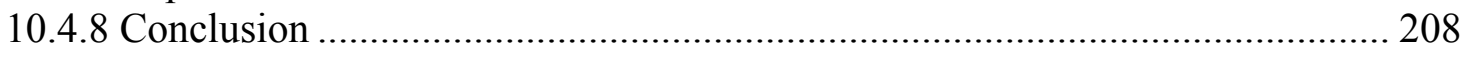

10.5 Microsoft Word Add-In Prototype Pilot Enhancements...................................... 209

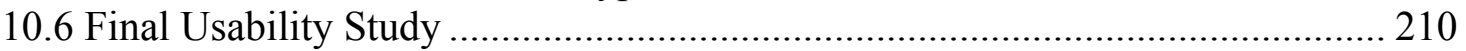

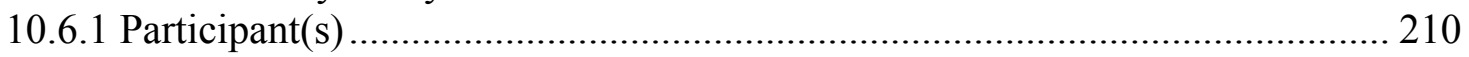

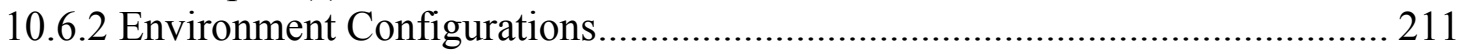

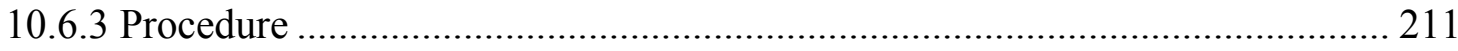

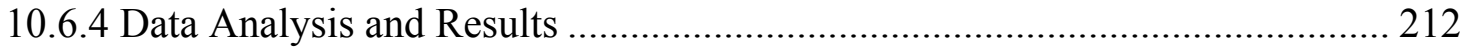

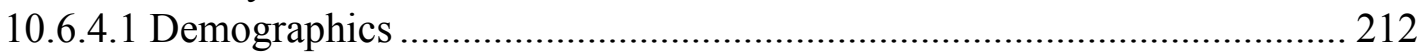

10.6.4.2 Prior Experiences with the Microsoft Word Interface.............................. 215

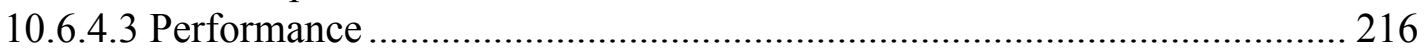

10.6.4.4 Analysis of the audio/video recordings ................................................... 218

10.6.4.5 Microsoft Word Add-In Prototype $-1^{\text {st }}$ Round Enhancements................ 220

10.6.4.6 Perception of Collaborative Writing Add-In Prototype.............................. 224

10.6.4.7 More Feedback Collected on Collaborative Writing Experiences ........... 227

10.6.4.8 Microsoft Word Add-In Prototype - ${ }^{\text {nd }}$ Round Enhancements. ............... 228

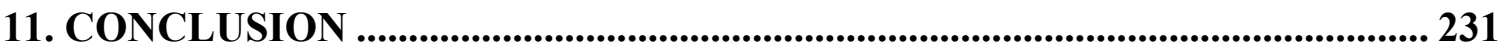

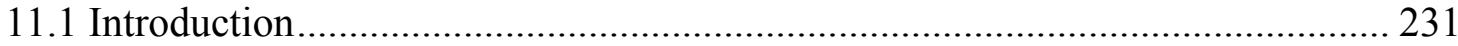

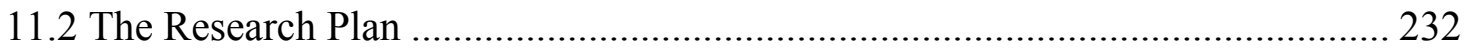

11.2.1 Preliminary Research Steps .................................................................... 232

11.2.2 Collaborative Writing and the Research Questions .................................... 233

11.2.3 The Baseline, Pilot and Additional Usability Studies.................................. 233

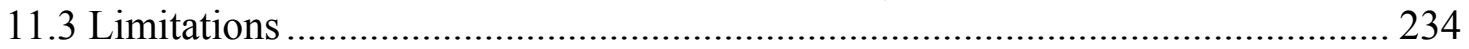

11.3.1 Preliminary Research Limitations ............................................................ 234

11.3.2 The Baseline, Pilot and Additional Usability Studies Limitations .............. 235

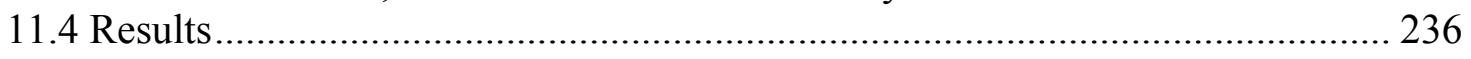

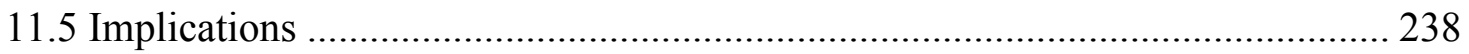

11.5.1 Preliminary Research .......................................................................... 238 
11.5.2 The Baseline, Pilot and Additional Usability Studies............................... 240

11.6 Future Research Directions............................................................................... 241

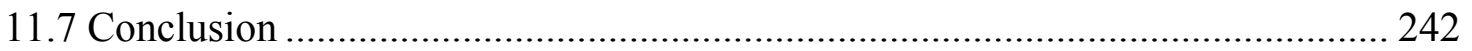

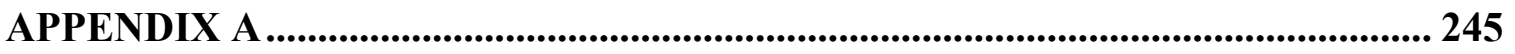

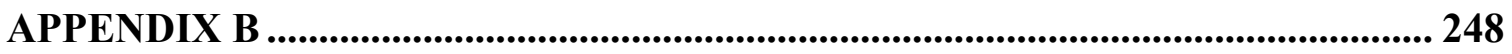

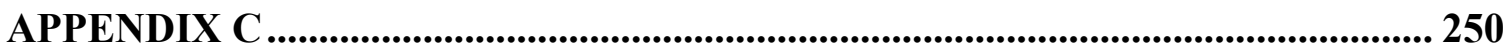

APPENDIX D .................................................................................................................... 256

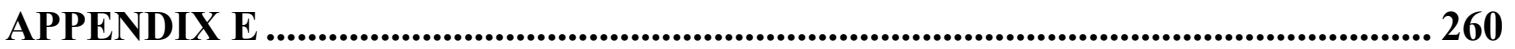

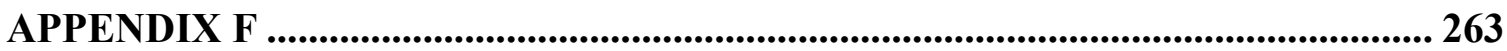

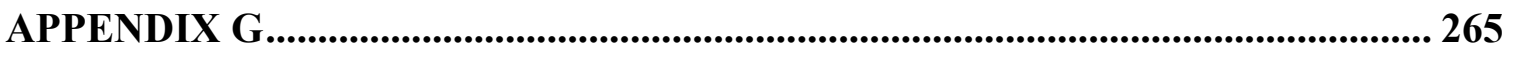

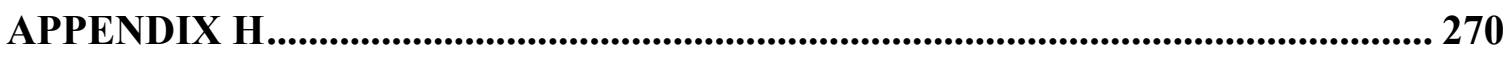

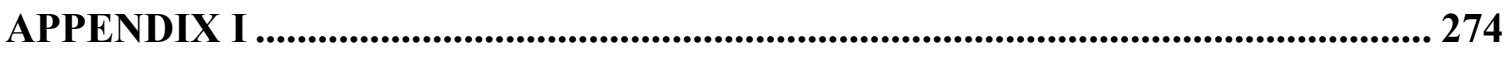

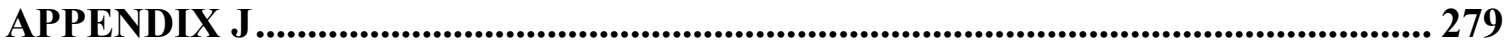

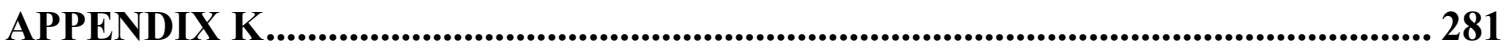

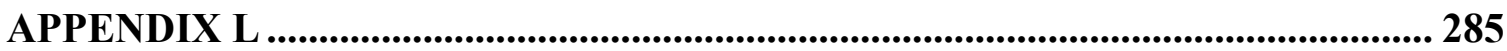

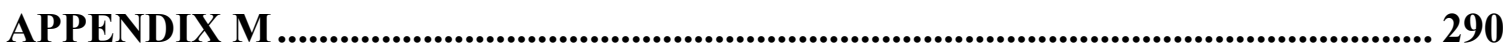

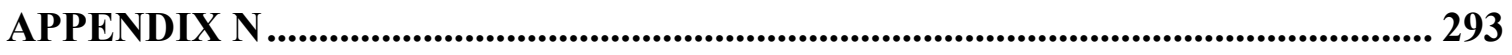

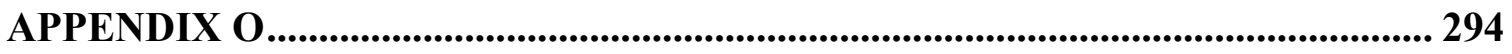

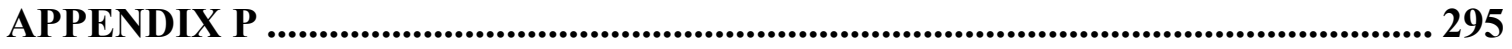

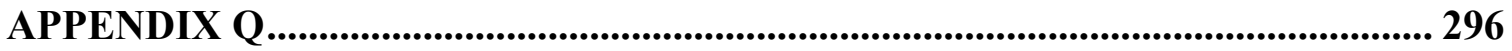

LIST OF REFERENCES ......................................................................... 297

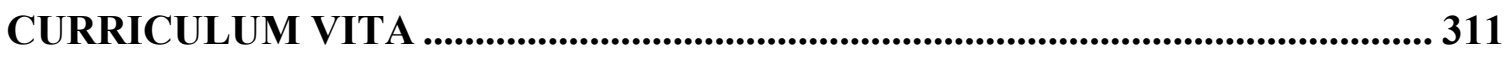




\section{LIST OF TABLES}

Table 1. Time and Place Matrix Categorization of Collaborative Technologies (Bishup et

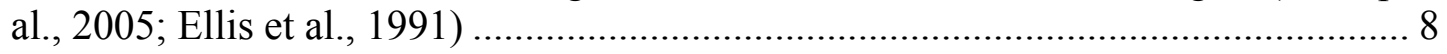

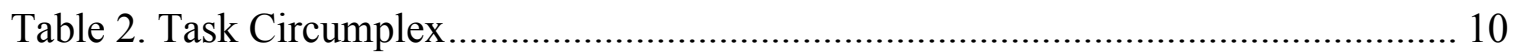

Table 3. Collaborative Technology Verses Other Types of Applications..................... 13

Table 4. Examples of Adaptive Technology for Persons with Disabilities................... 19

Table 5. Applications evaluated based on the Time and Place Matrix ........................... 27

Table 6. Fujitsu's Web Accessibility Inspector Evaluation Results .............................. 31

Table 7. (ATRC) Web Accessibility Checker Evaluation Results .................................. 33

Table 8. Web-based Accessibility Evaluations Summary ........................................... 38

Table 9. Collaborative Applications Comparison (shared criteria comparison) ............ 61

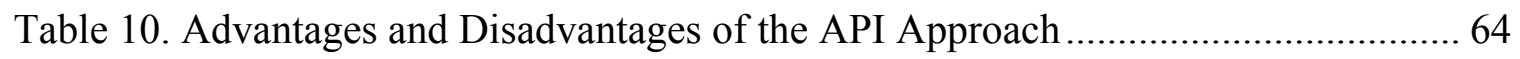

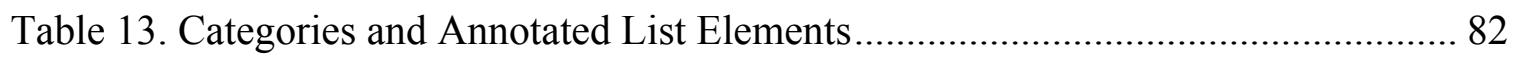

Table 14. Categories and Annotated List Elements................................................... 105

Table 15. Category and Annotated List Element .................................................. 128

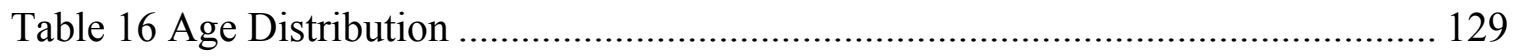

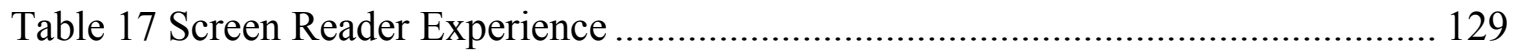

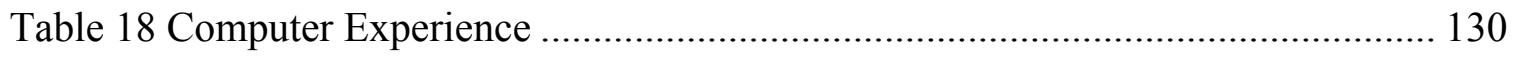

Table 19. Time and Place Categorization of Collaborative Technologies (Bishup et al.,

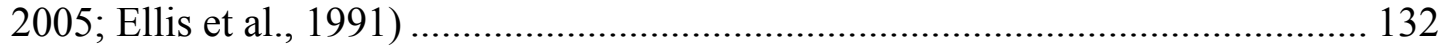

Table 20. Collaborative Purpose, Research Concerns and Questions ......................... 141

Table 21. Usability Study - Tasks Included by Application ..................................... 163

Table 22. Data Variables, Sources, Types, and Measurements .................................. 165

Table 23. Demographical Data - Visually Able Participants and Participants who are

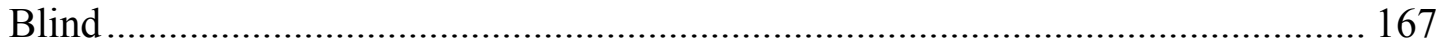

Table 24. Baseline Usability Study - Task Performance for Visaually Able Participants 170

Table 25. Baseline Usability Study - Task Performance for Participants Who Are Blind 171

Table 26. Category and Annotated List Element........................................................ 174

Table 27. Microsoft Word - Post-Test Questions/Statements by Visually able participants 178

Table 28. Microsoft Word - Post-Test Questions/Statements by Participants Who Are Blind.....

Table 29. Google Docs - Post-Test Questions/Statements by Visually able participants 182

Table 30. Feedback from Visually able participants regarding Microsoft Word ......... 184 
Table 31. Feedback from Participants Who Are Blind regarding Microsoft Word ...... 185

Table 32. Feedback from Visually able participants regarding Google Docs ................ 187

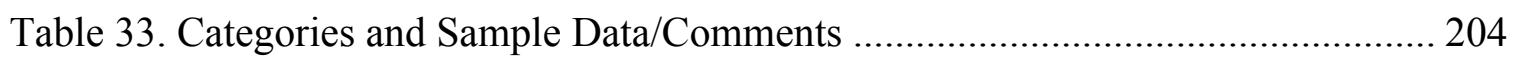

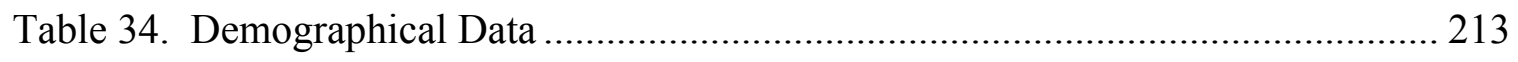

Table 35. Category and Element Examples ( $1^{\text {st }}$ round) ............................................. 219

Table 36. Perceptions of the Microsoft Word Add-In Prototype (1st round) ................. 227

Table 37. Perceptions of the Microsoft Word Add-In Prototype (2nd round) ............... 228

Table 38. Research Focus and Contributions …………............................................ 260

Table 39. Collaborative Accessibility Research Summary ………………………....... 263

Table 40. Collaborative Research Timeline and Published Works ................................. 290 


\section{LIST OF FIGURES}

Figure 1 Collaborative Technology Accessibility Evaluation Framework ...................... 25

Figure 2 WCAG 2.0 and its relationship to the 'A' rating scheme ("WCAG 2.0 @ the Pickards," 2009) 35

Figure 3 Microsoft Outlook Login Profile................................................................... 44

Figure 4 Microsoft Outlook's Folders and Panels ........................................................... 45

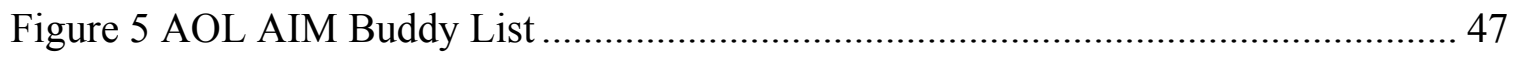

Figure 6 Twitter Interface and Menu (Home, Profile, Find People, Settings, Sign-out and

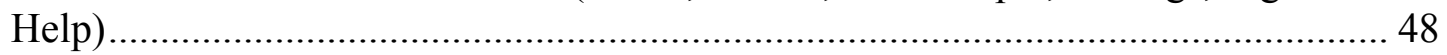

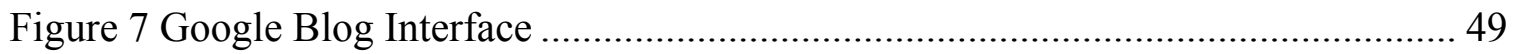

Figure 8 Google Mac Blog selected ...................................................................... 50

Figure 9 Google Mac Blog displayed .................................................................. 50

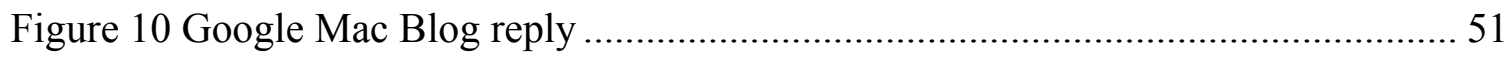

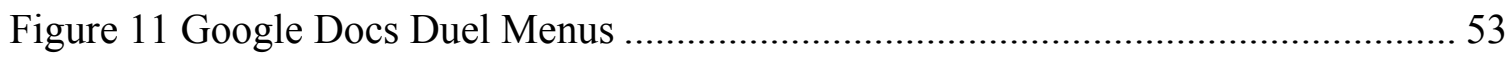

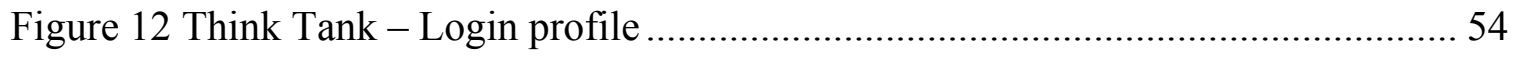

Figure 13 Think Tanks - Agenda, Categories, Ideas, and Comments............................. 55

Figure 15 Screen Reader Middleware Layer using WAI-ARIA ………............................ 65

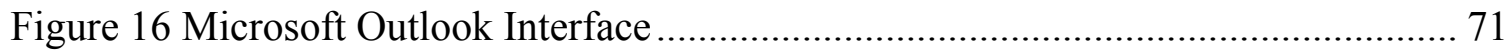

Figure 17 Microsoft Outlook with navigation and reading panes removed ..................... 72

Figure 18 Task Completion Times in Seconds ...................................................... 172

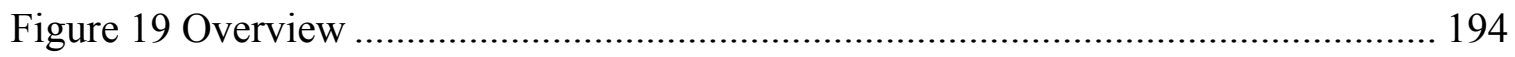

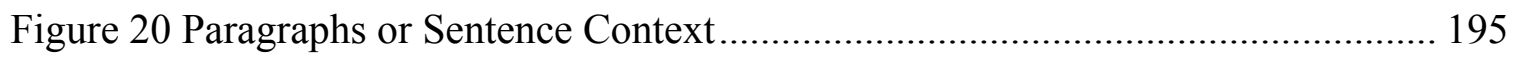

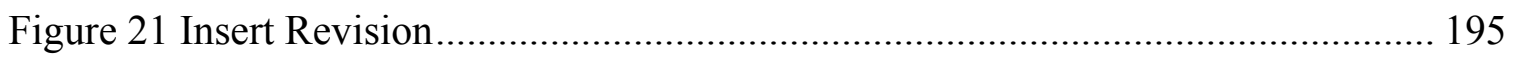

Figure 22 Revision View with selected revision (highlight in blue) ............................. 196

Figure 23 Microsoft Word Command Bar................................................................ 197

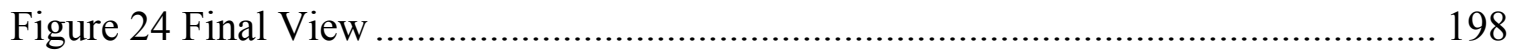

Figure 25 Overview of the document ........................................................................ 209

Figure 26 "Set View to Final" and "Set View to Revisions" Command Bar Options ... 209

Figure 27 Microsoft Word Add-In Prototype - Usability Study - Task Completion Mean

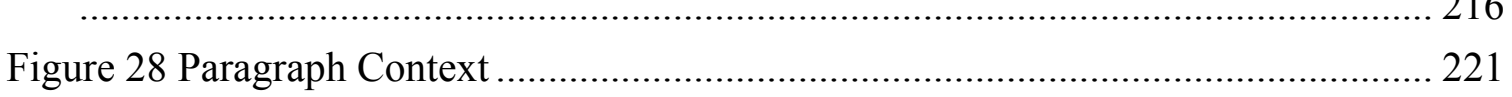

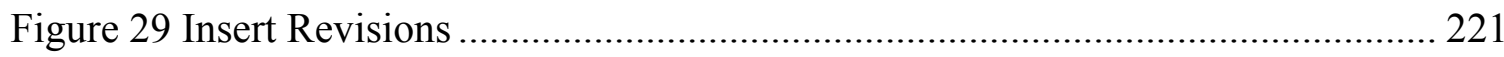

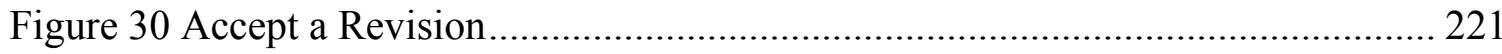




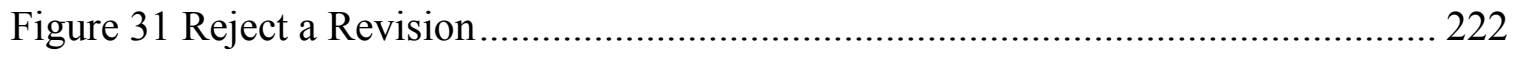

Figure 32 Repeat a Revision ................................................................................ 222

Figure 33 Analysis of Post-Study Questionnaire ( $1^{\text {st }}$ and $2^{\text {nd }}$ rounds) ......................... 224

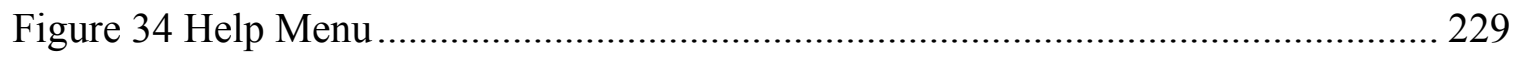

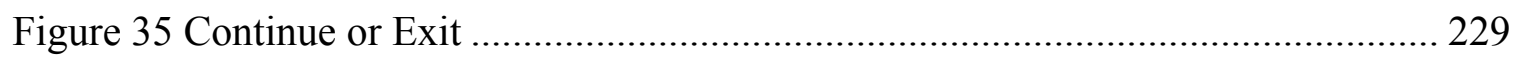

Figure 36 Microsoft Word Command Bar Hot Key Accesses .................................... 230 


\section{INTRODUCTION}

According to World Health Organization (WHO, 2013), about 285 million people are visually impaired worldwide, among them 39 million are blind. "Visual impairment or vision impairment is vision loss of a person to such a degree as to qualify as an additional support need through a significant limitation of vision capability resulting from either disease, trauma or congenital or degenerative conditions that cannot be corrected by conventional means, such as refractive correction or medication (Arditi \& Rosenthal, 1998)." On the other hand, "Total blindness is the complete lack of form and visual light perception and is clinically recorded as no light perception ("International Council of Ophthalmology," 2002)."

In today's global economy, collaboration is very important in business. With the trend of globalization today, it is more likely that a person in one organization will collaborate, communicate and coordinate with other persons in other locations, utilizing collaborative technologies to support this group collaboration (Shneiderman \& Plaisant, 2005). For example, people are dependent on a daily basis on email, newsgroups, discussion boards, teleconferences, blogs, wikis, Chat, and online communities. These are a few examples and are not intended as an exhaustive list.

Collaborative technologies (Shneiderman \& Plaisant, 2005) can be further classified as either groupware or other collaborative technologies that support group work. Groupware applications provide support to groups for an entire set of collaborative and communications tasks, and are self-contained and specific in purpose (Shneiderman \& Plaisant, 2005). For example, idea generation into a list, ranking or ordering the list 
and selecting the best idea from the list would be the tasks of a collaborative groupware decision support application.

Other collaborative technologies unlike full-fledged groupware applications focus on specific tasks for a general purpose (Shneiderman \& Plaisant, 2005). For example email, text messaging, document sharing, and calendar sharing as generalized collaborative services for the group to utilize.

Considering that the unemployment rate for working age persons who are blind in the United States is 70\% to 75\% ("U.S. Census Bureau," 2011a) and the prevalence of collaborative technologies (Shneiderman \& Plaisant, 2005), making these technologies accessible and usable becomes crucial.

The next few paragraphs present an overview of an approach to understand the access and use problems associated with collaborative technologies and a proposed solution for accessible collaborative writing for persons who are blind.

In order to establish a background and an understanding of the accessibility and usability issues persons who are blind encounter while collaborating, a literature review on collaborative technology, accessibility and usability (Schoeberlein \& Wang, 2009a), accessibility evaluations of web-based and Windows-based collaborative applications (Schoeberlein \& Wang, 2009b) and focus group studies were conducted (Schoeberlein \& Wang, 2011a, 2011b). At the conclusion of the literature review, accessibility evaluations, focus group studies and the field studies, accessibility and usability issues were identified in collaborative writing, email, Chat and group scheduling applications (Schoeberlein \& Wang, 2009a, 2009b, 2011a, 2011b). 
Collaborative writing was chosen as the research focus over email, Chat and scheduling collaborative technologies, since there had been little if any significant research conducted on the former technology versus the latter technologies.

The next step was to develop a formal plan to attack the accessibility and usability issues related to collaborative writing. The research design consisted of five distinct steps which included a baseline study of Microsoft Word and Google Docs, the development of an accessible collaborative writing prototype for Microsoft Word, a pilot usability study to examine the prototype and validate the study procedure and measurements, additional enhancements to the prototype and the study procedure and measurements, and a final set of usability studies to examine and improve the prototype for accessible collaborative writing. More details of the chapters to follow are presented in the next few paragraphs.

In order to establish a foundation of collaborative technologies and the skills and needs of persons who are blind, Chapter 2 begins with frameworks for categorizing types of collaborative technologies, followed by a review on various types of disabilities and the adaptive technologies utilized. The impact and uniqueness of collaborative technologies are discussed, to provide a reason for studying the access and use of such technologies in the work and school environments. Accessibility and usability are defined as they relate to persons with disabilities and the related adaptive technologies that facilitate accessibility and usability. This chapter ends with a discussion of the guidelines for Web accessibility.

The present state of the accessibility of collaborative applications is presented in Chapter 3, by evaluating several collaborative applications including Think Tank (“Group Systems, Inc.,” 2008), AOL AIM, Google Docs, Google Blog (2008), Microsoft 
Outlook (2008), Twitter (2009) and Microsoft SharePoint (2007). The evaluations utilized the Collaborative Technology Accessibility Evaluation Framework developed by the author of this dissertation.

Chapters 4 through 7 present the results from several field visits, field studies and focus group studies (Schoeberlein \& Wang, 2011) conducted at the National Federation of the Blind (NFB) ("National Federation of the Blind," 2008). The opinions of participants who are blind and the accessibility and usability issues they encounter while collaborating are presented.

Chapter 8 presents the list of possible research opportunities that were identified during the focus groups studies and the field studies. The participants of the focus group studies and the field studies reported collaborative writing technologies as being inaccessible and unusable. The participants deemed the tasks associated with collaborative writing essential to their daily work and therefore of great importance. Based on these outcomes, it was determined that collaborative writing would be the focus of this research.

The research methodology is presented in Chapter 9. The research focus was on collaborative writing. For this research, several usability studies were conducted in order to focus on identifying the accessibility and usability of Microsoft Word and Google Docs. The results of these usability studies were utilized to propose an interface design solution for collaborative writing.

Chapter 10 discusses the proposed design of the collaborative writing solution and the usability studies to evaluate this solution. Chapter 10 begins with the Baseline Usability Study, which presents the issues that people who are blind encounter while 
engaging in collaborative writing. The issues identified and the participants' feedback was utilized to develop an accessible and usable collaborative writing interface for Microsoft Word. Microsoft Word was selected as the collaborative writing application to improve, since it was identified as the primary collaborative writing application utilized by the participants of this research, and that Microsoft Word is a leading word processing application in the marketplace with over 90\% market share ("Microsoft Word Market share," 2013).

Based on the results of the baseline usability study, a Microsoft Word Add-In (2013) prototype was proposed to improve the accessibility and usability of track changes and comments features of Microsoft Word. A pilot usability study was conducted to examine the compatibility of the Microsoft Word Add-In prototype with the JAWS Screen Reader, and to make adjustments to the Microsoft Word Add-In prototype.

Once the Microsoft Word Add-In prototype was determined to be compatible with the JAWS Screen Reader, the final usability study was utilized to determine if the prototype improves the collaborative writing experience of persons who are blind.

The final chapter, Chapter 11, presents the implications and conclusion of this research. 


\section{RELATED LITERATURE}

\subsection{Collaborative Technology}

In order to establish a general background on collaborative concepts, this chapter begins with the definitions of collaborative terminology, followed by the Time/Place Framework (Table 1) (Bishup \& Roma, 2005; Ellis, Gibbs \& Reim, 1991) and the Task Circumplex (Table 2) (McGrath, 1984) for classifying collaborative technologies. The Time/Place Framework is utilized to determine whether the collaborators are at the same or different times and whether they are in the same physical location or in different locations. The Task Circumplex describes the types of tasks collaborators can participate in. For each type of task and time/place, the technologies applied for each case will be different to support the collaborations. Therefore, it is important to understand the type of tasks and time/place characteristics in order to properly support the collaborations between participants. The impact and uniqueness of collaborative technologies are discussed, to provide a reason for studying the need and use of such technologies in the work and school environments. Accessibility and usability are defined as they relate to persons with disabilities and the related adaptive technologies that facilitate accessibility and usability. This chapter ends with a discussion of the guidelines for Web accessibility.

\subsubsection{CSCW and Groupware}

Computer Supported Cooperative Work (CSCW) is the study of computer applications in support of communicating, coordinating, collaborating and competing tasks with persons working together in a shared time and space (Shneiderman \& Plaisant, 2005). 
Groupware applications are the technical applications providing CSCW solutions, to assist persons to communicate, coordinate, collaborate and compete (Shneiderman \& Plaisant, 2005), and allow persons in different locations to work together by collecting and sharing information (Umar, 1997, 373-380). Software applications that support telecommunications, multimedia document management, workflow management, and scheduling are just some examples (Umar, 1997, 373-380). For instance, a groupware application can support initial design, design approval and step-by-step assembly in a manufacturing process. A brain storming session, that involves idea generation, idea discussion, idea ranking, and final voting, can also be supported by a groupware application.

\subsubsection{Other Collaborative Technologies}

Unlike full-fledged groupware applications that support activities in a complete end-to-end group process, many applications are providing support by including collaborative tools that are often regarded as features in groupware applications (Shneiderman \& Plaisant, 2005). Email, text messaging, data or document sharing, and group calendars are some of the tools being integrated into software applications that are normally not considered groupware, but instead collaborative technologies (Shneiderman \& Plaisant, 2005). For instance, Microsoft Word provides document sharing, commenting and track changes that allows people to co-write articles; Microsoft Outlook has calendar, task, and appointment features to support group scheduling in addition to the support of email exchange. 


\subsubsection{Time and Place Framework}

There are many ways to categorize collaborative technologies in the literature. The most commonly cited framework is the time and place matrix (Bishup et al., 2005; Ellis et al., 1991) that distinguishes collaborative technologies (Shneiderman \& Plaisant, 2005) along two dimensions: whether the system supports groups working together at the same time (synchronous) or at different times (asynchronous); whether the system supports groups working together face-to-face or distributed. For example, a face-to-face group meeting in an electronic meeting facility using an Electronic Meeting System (EMS) while virtual teams with members distributed in different locations that have to work asynchronously will require the support from asynchronous collaborative technologies such as email, newsgroups, mailing lists, work flow automation systems, group calendar, and collaborative writing tools. Although started as desktop applications utilizing network connectivity, many collaborative systems are web-based implementations nowadays. Table 1 shows the categorizations of collaborative technologies using the time/place matrix.

Table 1. Time and Place Matrix Categorization of Collaborative Technologies (Bishup et al., 2005; Ellis et al., 1991)

\begin{tabular}{|l|l|l|}
\hline & Same Time (synchronous) & \multicolumn{1}{|c|}{$\begin{array}{c}\text { Different Times } \\
\text { (asynchronous) }\end{array}$} \\
\hline Same Place (local) & $\begin{array}{l}\text { Control rooms, } \\
\text { Wall projectors, } \\
\text { Using Electronic Meeting } \\
\text { Systems (EMS) in meeting } \\
\text { rooms }\end{array}$ & $\begin{array}{l}\text { Logs, } \\
\text { Team scheduling, } \\
\text { Group calendars }\end{array}$ \\
\hline $\begin{array}{l}\text { Different Places } \\
\text { (distributed) }\end{array}$ & $\begin{array}{l}\text { Chat, } \\
\text { Text messaging, } \\
\text { Video / audio conferencing }\end{array}$ & $\begin{array}{l}\text { E-mail, newsgroups, } \\
\text { discussion boards, } \\
\text { conferences, Blogs, Wikis, } \\
\text { Online communities }\end{array}$ \\
\hline
\end{tabular}




\subsubsection{Group Tasks}

Given that collaborative technologies support a variety of group tasks, it is important to understand the types of tasks typical in a group environment.

One approach to identify different types of tasks is to utilize the Task Circumplex, developed by McGrath (1984). Depending on the type of the task, different levels of communication and task support may be requested by the group. The Task Circumplex can be utilized as a framework to identify the type of task and the application support needed for the identified task. Table 2 presents the Task Circumplex by presenting one sample task with possible collaborative technology support for each quadrant. Group Systems' Think Tank (2008) is a decision support application that collaborators can utilize to develop and prioritize ideas into a workable plan. Think Tank is utilized as an example for the various task types in the Task Circumplex Table (Table 2). There are other available applications that provide decision support. Think Tank is one example and not considered an exhaustive list. 
Table 2. Task Circumplex

\begin{tabular}{|c|l|l|}
\hline \multicolumn{1}{|c|}{ Task Type } & \multicolumn{1}{|c|}{ Task } & \multicolumn{1}{c|}{ Groupware Example } \\
\hline Generate & $\begin{array}{l}\text { Planning task to generate } \\
\text { plans; and, creativity tasks to } \\
\text { generate ideas. }\end{array}$ & $\begin{array}{l}\text { Group Systems' Think } \\
\text { Tank's lists for category } \\
\text { generation, idea generation, } \\
\text { and comments are available } \\
\text { to assist in generating and } \\
\text { voting on ideas. }\end{array}$ \\
\hline Choose & $\begin{array}{l}\text { Intellectual use for problem } \\
\text { solving for a correct answer } \\
\text { and decision-making tasks for } \\
\text { find a preferred answer. }\end{array}$ & $\begin{array}{l}\text { Group Systems' Think } \\
\text { Tank's list of choices can be } \\
\text { utilized for sorting choices } \\
\text { by priority based on voting } \\
\text { results. }\end{array}$ \\
\hline Negotiate & $\begin{array}{l}\text { Cognitive conflict for } \\
\text { resolving conflicts; and, } \\
\text { mixed-motor tasks to resolve } \\
\text { conflicts of motive-interest. }\end{array}$ & $\begin{array}{l}\text { Group Systems' Think } \\
\text { Tank's lists of categories and } \\
\text { ideas can be utilized to } \\
\text { negotiate between parties. }\end{array}$ \\
\hline Execute & $\begin{array}{l}\text { Contest battles for conflicts of } \\
\text { power and competitions; and, } \\
\text { performances compared } \\
\text { against objective standards. }\end{array}$ & $\begin{array}{l}\text { Microsoft Project's tasks, } \\
\text { milestones, PERT Charts, } \\
\text { and GANTT Charts can be } \\
\text { utilized for planning and } \\
\text { performance evaluations. }\end{array}$ \\
\hline
\end{tabular}

The "generate" quadrant refers to tasks associated with plan development and idea generation (McGrath, 1984). Plan development may involve developing an outline of all of the tasks in the plan; adding details to each task in the plan; arranging the tasks based on order or precedence; and, assigning persons to each task in the plan, etc. On the other hand, generating ideas includes listing, categorizing, organizing/sorting, and prioritizing the ideas to solve an issue or problem. For example, Group System's Think Tank (2008) provides group members the capabilities to categorize, organize and prioritize ideas that are generated within the group. 
The "choose" quadrant pertains to tasks for choosing a correct or best answer by listing all possible answers to a particular problem being addressed, organizing the answers from best to worst, voting on the best or correct answer, and choosing the correct or best answer based on the voting results (Grounded Theory, 2009). Supporting these tasks includes email and Chat to communicate answers, and decision support for voting and selecting. Group Systems' Think Tank (2008) can be utilized to provide support to the group members for developing a list of choices that can be sorted by priority based on voting results.

For the "negotiate" quadrant, tasks focus on resolving conflicts through negotiations (Grounded Theory, 2009). Identifying items under negotiation, categorizing the items under negotiation as satisfied or not satisfied, discussing items remaining to be satisfied, and proposing an agreeable solution are some of the tasks associated with negotiating. Email and Chat collaborative technologies may be used to communicate satisfied and unsatisfied items to negotiating parties.

The "execute" quadrant includes tasks associated with competitions and performances (Grounded Theory, 2009). Setting performance goals, identifying performance milestones to achieve goals, comparing results to goals, and reporting status are the types of tasks associated with competitions and performances. Project management tools are best suited for these types of tasks. Microsoft Project can be utilized by group members to track performance of tasks against expected completion times.

The Task Circumplex (Table 2) was designed so that the tasks are mutually exclusive between categories, collectively exhaustive, logically related, and useful for 
comparing similarities and differences of various tasks used for group research. However, it does not provide a means to distinguish the tasks between quadrants. Therefore, the identification of the quadrant a task belongs may not always be a clear-cut answer. Instead, tasks that seem to have covered multiple quadrants are often present while working in groups. Researchers need to be cautious when determining the level of support needed for those tasks.

\subsubsection{The Impact of Collaborative Technologies}

Collaborative Technologies impact how groups of persons communicate, coordinate, collaborate, and compete (Shneiderman \& Plaisant, 2005). For example, Chat enables people to have conversations regardless of the physical location of the persons involved in the conversation (Shneiderman \& Plaisant, 2005). Email allows electronic mail delivery anywhere and anytime throughout the world almost instantaneously (Shneiderman \& Plaisant, 2005). Scheduling applications, that support group calendars, provide meeting participants the ability to coordinate their activities with other participants (Shneiderman \& Plaisant, 2005). Decision-support applications such as Group Systems' Think Tank (2008) support anonymous group decision-making in a facilitated electronic meeting environment from any location (Shneiderman \& Plaisant, 2005). Essentially, collaborative technologies have removed the barriers of distance, location and time, when communicating, coordinating, collaborating and competing (Shneiderman \& Plaisant, 2005). 


\subsubsection{The Uniqueness of Collaborative Technologies}

Compared to other types of applications, collaborative technologies are unique based on certain aspects of the application including: type of work orientation; number of users supported; network support; communication mode; floor control; and, concurrency control (Table 3) (Shneiderman \& Plaisant, 2005). This is not an exhaustive list, but the items considered for comparison purposes.

Table 3. Collaborative Technology Verses Other Types of Applications.

\begin{tabular}{|l|l|l|l|}
\hline \multicolumn{1}{|c|}{ Aspect } & \multicolumn{1}{|c|}{$\begin{array}{c}\text { Collaborative } \\
\text { Technology }\end{array}$} & \multicolumn{1}{|c|}{$\begin{array}{c}\text { Stand-Alone } \\
\text { Windows } \\
\text { Application }\end{array}$} & \multicolumn{1}{|c|}{$\begin{array}{c}\text { Traditional Web } \\
\text { Page }\end{array}$} \\
\hline $\begin{array}{l}\text { Type of Work } \\
\text { Orientation }\end{array}$ & $\begin{array}{l}\text { Shared set of } \\
\text { tasks of an end- } \\
\text { to-end process }\end{array}$ & Single user tasks & Single user tasks \\
\hline $\begin{array}{l}\text { Number of Users } \\
\text { Supported }\end{array}$ & Multi-user & Single-user & Single-user \\
\hline Network Support & Networked & Stand-alone Desktop & Networked \\
\hline $\begin{array}{l}\text { Communication } \\
\text { Mode }\end{array}$ & $\begin{array}{l}\text { Collaborative, } \\
\text { asynchronous and } \\
\text { synchronous }\end{array}$ & $\begin{array}{l}\text { Single-user, stand- } \\
\text { alone }\end{array}$ & $\begin{array}{l}\text { Single-user, stand- } \\
\text { alone }\end{array}$ \\
\hline Floor Control & Necessary & Not necessary & Not necessary \\
\hline $\begin{array}{l}\text { Concurrency } \\
\text { Control }\end{array}$ & Necessary & Not necessary & Not necessary \\
\hline
\end{tabular}

\subsubsection{Type of Work Orientation}

Collaborative technologies that are considered groupware provide support for complete end-to-end processes such as brainstorming, negotiating, and consensus building (Shneiderman \& Plaisant, 2005). For example, in a brainstorming process the tasks might include selecting a topic to discuss, adding content to the topic, prioritizing the content of the topic and summarizing the content to formulate a conclusion. The specialized nature of a group task, like brainstorming, makes groupware applications 
unique in regard to application development. The application must follow a workflow in order for the tasks of a brainstorming session to make sense to the participants and to reach completion. Before a final solution is found, there may be multiple rounds of idea generation, categorization, organization and elimination.

Other collaborative technologies such as email, Chat and collaborative writing provide support for a shared group task that is not constrained by a specific process (Shneiderman \& Plaisant, 2005). Microsoft Word's co-editing track changes feature could be considered an application that supports group work and is not associated with a specific process.

Stand-alone windows applications such as Microsoft Office and Microsoft Access support single-user tasks. However, these applications may have co-editing or sharing features that can be utilized by groups, so these applications can be utilized to support group work if a group shares the application.

\subsubsection{Number of Users Supported}

Collaborative Technologies are unique in regard to the number of users the application can concurrently support (Shneiderman \& Plaisant, 2005). For these applications, multiple users can interact with each other using the same application concurrently through a communications channel established by the application; whereas, stand-alone Windows-based applications do not support multiple user interactions. 


\subsubsection{Network Support}

Collaborative technologies are set apart from other types of applications by requiring some network architecture in order to provide group collaborations and communications (Shneiderman \& Plaisant, 2005). Stand-alone Windows-based applications do not require network connectivity, so they tend to be less complex. Web pages do require network connectivity to search various web sites and to provide the communication channels to various web sites. The communications necessary to search the Internet are not as complex as the communications necessary to support group activities associated with collaborative applications. To search the Internet, single-user requests are sent and responses are returned. For group activities associated with collaborative applications, multiple concurrent communication channels are established so multiple users can communicate, collaborate, coordinate and compete.

\subsubsection{Communications Mode}

Collaborative technologies support both synchronous and asynchronous communications between multiple users and the application (Shneiderman \& Plaisant, 2005); whereas, stand-alone Windows-based applications and traditional Web pages generally provide single communications between an end user and the application.

\subsubsection{Floor Control, Concurrency Control}

Floor control is concerned with which party in the communication is actively communicating a message or which party's turn is active. For example, using America Online Instant Messenger (AOL IM) (“AOL Company Overview,” 2009) Chat in a 
conversation, each participant in the conversation has a current cursor that they can type text messages into and send the text message to the recipient. The cursor then is positioned for a reply from the recipient. This interleaving of messages is one technique of floor control utilized in synchronous communications.

Concurrency control is concerned with shared documents in which two or more persons are attempting to access the same document content at the same time. Google Docs (2009) supports co-editing of a shared document between two or more persons and provides concurrency control by locking the entire document while it is being edited for changes. While one person is editing the document, the other persons are temporarily locked-out from editing the document. After the document changes are saved, the document is unlocked and available for editing by any person.

\subsection{Accessibility and Usability}

Accessibility describes the degree to which a product, device, service, or environment is accessible by as many people as possible (“Accessibility," 2013). It is often used to focus on people with disabilities and their right of access to entities, often through the use of assistive technology (“Adaptive Technology” 2008). In order to understand the output from computer systems, persons who are blind are forced to use either tactile displays or sound for output. A screen reader such as Freedom Scientifics' JAWS (2008) is a piece of software that runs in the background to read the web page or screen code and send any text it finds there to a speech synthesizer (Hampel, Keil-Slawik, Claassen, Plomnann \& Reimann, 1999). However, the screen reader cannot interpret graphical information on the screen, if it is not labeled properly. Java-based technologies such as Chat rooms are often inaccessible to persons who cannot use a mouse. Poorly 
labeled forms and the use of frames can also make the program inaccessible to persons who are blind. Moreover, with technology advancement, more and more applications are incorporating dynamically generated content, which presents accessibility challenges to persons who are blind when not marked up properly.

On the other hand, usability refers to the extent a product (e.g. device or service) can be utilized by specific users to achieve specific goals with effectiveness, efficiency and satisfaction in a specific context of use ("Usability," 2013).

Adaptive technologies (“Adaptive Technology," 2008) provide support for typing assistance, hearing assistance, speech assistance, etc., for persons with disabilities. The World Wide Web - Web Content Accessibility Guidelines (W3C-WCAG) establishes accessibility guidelines for Web-based applications (“W3C HTML 1.0,” 2008; "W3C HTML 2.0,” 2009). However, Windows-based accessibility guidelines are not fully developed, but there are some attempts at providing interface features in the form of accessible controls (i.e. properties such as: accessible description, accessible name, and accessible role), developed by Microsoft Corporation ("Microsoft Corporation: Properties on Windows Forms Controls That Supports Accessibility Guidelines," 2011). In order to support accessibility and usability, a fundamental awareness of the adaptive technologies and the accessibility needs of persons with disabilities are necessary.

\subsubsection{Persons with Disabilities}

Many types of disabilities have been discussed in literature which includes persons who are blind or visually impaired, persons who are deaf or hearing impaired, persons with speech impairments, persons with coordination, dexterity or mobility impairments, and persons with cognitive disabilities (“Adaptive Technology,” 2008). 
Persons who are blind with no residual vision or are visually impaired with vision loss to such a degree as to qualify for additional support (Arditi \& Rosenthal, 1998) are not able to see the user interface. Those who are deaf or hearing impaired cannot hear the audio signals of the user interface's speakers. Persons with speech impairments are not able to interact with speech recognition technologies found with user interfaces. Persons with coordination, dexterity or mobility impairments are not able to interact with some forms of input devices such as the keyboard or the mouse. Those with cognitive disabilities may have trouble reading and understanding content. Therefore, some persons with disabilities need additional support, in the form of adaptive technologies, in order to interact with computer applications.

\subsubsection{Adaptive Technologies Available to Persons with Disabilities}

Computer users and persons with disabilities may have difficulties in the following three general areas that have to be overcome in order to interact effectively with computer applications in general and collaborative technologies specifically (Schoeberlein \& Wang, 2011a). The three areas are: providing input, perceiving output, and understanding how to use the computer (Schoeberlein \& Wang, 2011a). These three areas can be seen as a hierarchy of skills needed to interact with and use a computer effectively.

Various adaptive technologies (Table 4) are available to assist persons with disabilities provide input and output support. These technologies have been developed to provide access support in the form of vision assistance, speech assistance, reading assistance and typing assistance for persons with disabilities. 
Table 4. Examples of Adaptive Technology for Persons with Disabilities.

\begin{tabular}{|c|c|c|}
\hline Disability & $\begin{array}{c}\text { Adaptive Technology Input } \\
\text { Support }\end{array}$ & $\begin{array}{c}\text { Adaptive Technology Output } \\
\text { Support }\end{array}$ \\
\hline Blind & $\begin{array}{l}\text { Speech recognition software - } \\
\text { typing assistance (“Speech } \\
\text { Recognition," 2008). } \\
\text { Braille writing devices - typing } \\
\text { assistance. }\end{array}$ & $\begin{array}{l}\text { Screen readers and Braille } \\
\text { displays - reading assistance. }\end{array}$ \\
\hline $\begin{array}{l}\text { Visually } \\
\text { impaired - } \\
\text { low vision }\end{array}$ & $\begin{array}{l}\text { Speech recognition software - } \\
\text { typing assistance (“Speech } \\
\text { Recognition,” 2008). } \\
\text { Braille writing devices - typing } \\
\text { assistance. }\end{array}$ & $\begin{array}{l}\text { Screen readers - reading } \\
\text { assistance. } \\
\text { Braille displays, Enlarged images } \\
\text { - reading and vision assistance. } \\
\text { Enlarged text - output reading } \\
\text { and vision assistance. }\end{array}$ \\
\hline $\begin{array}{l}\text { Deaf and } \\
\text { hearing } \\
\text { impaired }\end{array}$ & $\begin{array}{l}\text { Telephone typewriter or } \\
\text { teletypewriter devices (TTY/TTD) } \\
\text { - typing assistance } \\
\text { ("Telecommunications Devices," } \\
\text { 2008). }\end{array}$ & $\begin{array}{l}\text { Closed captions - reading } \\
\text { assistance ("Closed Caption," } \\
\text { 2008). } \\
\text { American Sign Language } \\
\text { Interpretation. }\end{array}$ \\
\hline $\begin{array}{l}\text { Speech } \\
\text { impairments }\end{array}$ & & $\begin{array}{l}\text { Augmentative and Alternative } \\
\text { Communication (AAC) } \\
\text { ("Augmentative and Alternative } \\
\text { Communication," 2013). } \\
\text { Speech synthesizers - speech } \\
\text { assistance ("Speech Synthesis," } \\
\text { 2008). }\end{array}$ \\
\hline $\begin{array}{l}\text { Coordination, } \\
\text { dexterity and } \\
\text { mobility } \\
\text { impairments }\end{array}$ & $\begin{array}{l}\text { Speech recognition software - } \\
\text { typing assistance ("Speech } \\
\text { Recognition," 2008). }\end{array}$ & \\
\hline $\begin{array}{l}\text { Cognitive or } \\
\text { learning } \\
\text { disabilities, } \\
\text { like dyslexia }\end{array}$ & $\begin{array}{l}\text { Speech recognition software - } \\
\text { typing assistance ("Speech } \\
\text { Recognition," 2008). } \\
\text { Touch Typing Tutors (“Assistive } \\
\text { Technologies for Persons with } \\
\text { Dyslexia,” 2011). }\end{array}$ & $\begin{array}{l}\text { Speech synthesizers - speech } \\
\text { assistance ("Speech } \\
\text { Recognition," 2008). } \\
\text { Scanning pens ("Assistive } \\
\text { Technologies for Persons with } \\
\text { Dyslexia," 2011). }\end{array}$ \\
\hline
\end{tabular}


Persons with disabilities perceive the current state of components and events presented by interacting with adaptive technologies. For example, the current state of a component (checkbox) on the interface may be determined by using a screen reader to read the components attributes. Events may be more difficult to perceive, since many events (message boxes, Chat messages) are often missed when using adaptive technologies such as screen readers (Mikovec, Vystrcil, \& Slavik, 2009; Buzzi, Buzzi, Leporini \& Senette, 2008). In addition to adaptive technologies, Web Accessibility Initiative - Accessible Rich Internet Applications (WAI-ARIA) Metadata ("W3C," 2006) may be needed to assist persons with disabilities perceive events when interacting with active components on Web pages. Unfortunately, WAI-ARIA Metadata is not available for event management of Windows applications.

\subsubsection{Guidelines for Web Accessibility}

Guidelines for Web accessibility have been developed in regard to accessibility for persons with any number of disabilities: visually impaired; cognitively impaired (less focus in the guidelines); motor difficulty; deafness; seizures, etc. 
The following Web accessibility guidelines are proposed and adopted today:

- U.S. Government's Section 508, of the Rehabilitation Act ("Rehabilitation Act, Section 508,"1998).

- World Wide Web - Web Content Accessibility Guidelines, W3C WCAG 1.0 (“W3C," 2000), and W3C WCAG 2.0 (“W3C HTML,” 2009).

- The United Kingdom's Special Education Needs and Disabilities Act, SENDA, requires "reasonable adjustments" ("Special Education Needs and Disabilities Act," 2008).

- Web Accessibility Initiative - Accessible Rich Internet Applications, WAI-ARIA, for Web page live regions (“W3C,” 2006).

- Authoring Tool Accessibility Guidelines 2.0 (ATAG) (“Authoring Tool Accessibility Guidelines," 2013) for the development of accessible web authoring tools provide accessible pre-authoring content, accessible templates for web authoring, and validation for WCAG 2.0 accessibility of created content. Provides repair suggestion for content failing WCAG 2.0 Guidelines.

- The National Federation of the Blind's guidelines for web application developers (Chong, 2008; "National Federation of the Blind," 2008). 


\subsubsection{Guidelines for Windows Applications}

Collaborative technologies may be developed as Web applications or traditional console applications (i.e. VT-100 text-based terminals) or Windows applications (i.e. Microsoft Windows). Accessibility guidelines are available for traditional console applications and Windows applications too ("Section 508," 2013).

\subsection{Conclusion}

Adaptive technologies provide support for persons with disabilities to gain access and utilize computer applications. Accessibility guidelines have been developed to help develop and evaluate applications for accessibility. Therefore, it is important to have an understanding of both adaptive technologies and accessibility guidelines in order to design and implement accessible and usable collaborative applications. 


\section{TECHNOLOGY EVALUATION AND APPLICATION COMPARISION}

\subsection{Introduction}

In order to gain a better understanding of the present state of collaborative technology accessibility, an evaluation of several collaborative applications was conducted. The recent literature presented general Web Content Accessibility Guidelines ("WCAG 2.0 @ the Pickards," 2009), WAI-ARIA and U.S. Section 508 of the Rehabilitation Act for evaluating accessibility. Additionally, accessibility evaluation criteria were developed for Web, console and Windows applications, for the purpose of evaluating collaborative applications for accessibility. The next sections present the evaluations for accessibility of the following applications: Think Tank ("Group Systems, Inc.," 2008); AOL AIM; Google Docs; Google Blog (2008); Microsoft Outlook (2008); Twitter (2009); and, Microsoft SharePoint (2007), utilizing the Web, console and Windows accessibility evaluation criteria presented in this chapter.

\subsection{Collaborative Technology Accessibility Evaluation Framework}

The Collaborative Technology Accessibility Evaluation Framework (Figure 1), developed by the author, presents the hierarchy of the accessibility evaluation process. First, Web-based collaborative applications are automatically evaluated against WCAG 2.0 (“WCAG $2.0 @$ the Pickards,” 2009), using the Adaptive Technology Resource Center (ATRC) Web Accessibility Checker tool (“ATRC Web Accessibility Checker,” 2009). Second, these applications are evaluated for usability by manually working through specific tasks utilizing just the keyboard interface and assessing whether the 
application is accessible and usable. Additionally, the application is evaluated to determine if the application contains integrated (built-in) characteristics (text-to-Braille, speech recognition) and API interfaces that could be utilized to build accessible interface prototypes. Finally, if persons with disabilities are available have them perform common tasks using each collaborative application to determine accessibility and usability. 


\begin{tabular}{|c|c|c|}
\hline $\begin{array}{l}\text { 1. Web-Based } \\
\text { Collaborative } \\
\text { Technology } \\
\text { Evaluation }\end{array}$ & $\begin{array}{l}\text { Utilize Web } \\
\text { Accessibility Tool } \\
\text { (ATRC Web } \\
\text { Accessibility } \\
\text { Checker) }\end{array}$ & $\begin{array}{l}\text { Does the } \\
\text { Collaborative } \\
\text { Application meet } \\
\text { WCAG 2.0? }\end{array}$ \\
\hline $\begin{array}{l}\text { 2. Web-based, } \\
\text { Console-based } \\
\text { (VT-100), or } \\
\text { Window-based } \\
\text { (Microsoft } \\
\text { Windows) } \\
\text { accessibility } \\
\text { and usability } \\
\text { Collaborative } \\
\text { Technology } \\
\text { Evaluation }\end{array}$ & $\begin{array}{l}\text { Evaluate for } \\
\text { integrated speech } \\
\text { recognition, text-to- } \\
\text { Braille, keyboard } \\
\text { access, and API } \\
\text { availability } \\
\text { Perform keyboard- } \\
\text { only accessibility } \\
\text { and usability checks } \\
\text { by performing } \\
\text { common tasks } \\
\text { supported by the } \\
\text { Collaborative } \\
\text { Annlication }\end{array}$ & $\begin{array}{l}\text { Is the } \\
\text { Collaborative } \\
\text { Application } \\
\text { accessible and } \\
\text { usable? } \\
\text { Is an API Library } \\
\text { available to create } \\
\text { an accessible } \\
\text { collaborative } \\
\text { interface prototype } \\
\text { that can be } \\
\text { evaluated in an } \\
\text { accessibility and } \\
\text { usahilitv studv? }\end{array}$ \\
\hline $\begin{array}{l}\text { 3. Field } \\
\text { Collaborative } \\
\text { Tech Access } \\
\text { and Use } \\
\text { Evaluation by } \\
\text { persons with } \\
\text { disabilities }\end{array}$ & $\begin{array}{l}\text { Perform } \\
\text { accessibility and } \\
\text { usability checks by } \\
\text { performing common } \\
\text { tasks supported by } \\
\text { the Collaborative } \\
\text { Application }\end{array}$ & $\begin{array}{l}\text { Is the } \\
\text { Collaborative } \\
\text { Application } \\
\text { accessible and } \\
\text { usable based on a } \\
\text { target user group } \\
\text { evaluation? }\end{array}$ \\
\hline
\end{tabular}

Figure 1 Collaborative Technology Accessibility Evaluation Framework 
3.3 Applications for Evaluation Selection

Because of the wide variety of collaborative applications available, it is not feasible to exhaust. Therefore, the following factors were considered when selecting applications for this study:

- Category that the application falls into: at least one collaborative application in each time/place category to be evaluated. However, since most of the applications that supports collaboration from different locations also can be utilized for colocation collaboration, emphasis was given to applications that support distributed group collaboration.

- Type of interface: Although most of the applications are now on the Web, there are still numerous console and Windows applications. Comparing the accessibility of Web and Windows applications will help identify current problems associated with interface design. Therefore, Web, Windows, and console applications were identified for this study.

- Popularity of the application: investigate the application that has a large number of users for the application can be regarded as representative of that type of application.

- Availability of the tools: There are some commercial collaborative applications that claim to have a wide range of features. Unfortunately, an evaluation copy was not available to determine their accessibility. 
As representatives of a broader range of collaborative applications, Microsoft Outlook, Microsoft SharePoint, America Online Instant Messenger (AIM), Google Blog, Twitter, Google Docs, and Group Systems' Think Tank were chosen (Table 5). These tools were selected since they represent a mix of features that support group work, such as email, scheduling, Chat, blog, document sharing, and group decision-making. All of the selected applications are considered distributed based on the time-place matrix (Table 1), since they are primarily accessed from different locations. However, the selected applications can be accessed locally too. Distributed applications allow a focus on issues related to persons working in isolation and on asynchronous and synchronous communications (i.e. floor control; and, concurrency control) from different locations.

Distributed applications that support synchronous communications include AOL AIM; and, Group Systems' Think Tank. While distributed applications that support asynchronous communications include Microsoft Outlook, Microsoft SharePoint, Google Docs, Google Blog and Twitter.

Table 5. Applications evaluated based on the Time and Place Matrix

\begin{tabular}{|l|l|l|}
\hline & $\begin{array}{c}\text { Same Time } \\
\text { (synchronous) }\end{array}$ & $\begin{array}{c}\text { Different } \\
\text { Times } \\
\text { (asynchronous) }\end{array}$ \\
\hline $\begin{array}{l}\text { Same Place } \\
\text { (local) }\end{array}$ & & \\
\hline $\begin{array}{l}\text { Different } \\
\text { Places } \\
\text { (distributed) }\end{array}$ & $\begin{array}{l}\text { AOL AIM, } \\
\text { Group Systems' } \\
\text { Think Tank, } \\
\text { Google Docs, } \\
\text { Microsoft } \\
\text { SharePoint }\end{array}$ & $\begin{array}{l}\text { Microsoft } \\
\text { Outlook email, } \\
\text { Google Blog, } \\
\text { Twitter, Google } \\
\text { Docs, Microsoft } \\
\text { SharePoint }\end{array}$ \\
\hline
\end{tabular}


After the applications for evaluation were identified, they were assessed in respect to their interface type (Web, Windows, or console), using the Collaborative Technology Accessibility Evaluation Framework (Figure 1).

The next sections will present evaluations of Web applications using automated tools for WCAG compliance. Following these automated evaluations, Web, Windows and Console applications will be evaluated by a usability evaluation.

\subsection{Automatic Accessibility Evaluations}

The next sub-sections will discuss the criteria used to evaluate Web applications that support group work; the procedure used to evaluate these applications; the evaluations using Fujitsu's Accessibility Inspector (2008) and ATRC Web Accessibility Checker (2009). Fujitsu's Accessibility Inspector (2008) was selected due to its availability and ease of use. ATRC Web Accessibility Checker (2009) was selected, since it was one of the few accessibility checkers available at the time of the evaluation to evaluate WCAG 2.0 and was easy to use. However, no automatic accessibility tool is completely accurate and may produce false positives. Therefore, it is essential to manually check the results.

\subsubsection{Web-based Applications' Evaluation Criteria.}

For Web applications, Web Content Accessibility Guidelines (WCAG) ("WCAG $2.0 @$ the Pickards,”2009) and the Web Accessibility Initiative's Accessible Rich Internet Applications (WAI-ARIA) guidelines ("W3C," 2006) were identified as necessary for developing and evaluating accessible web-based collaborative applications. These guidelines include requirements regarding web-page presentation of content and 
are the foundations for evaluating web-accessibility using an accessibility tool, like ATRC Web Accessibility Checker (2009) for WCAG 2.0 compliance. Compliance of WCAG Guidelines can be met by following the guidelines for either version 1.0 or 2.0; however, as new Web pages are developed or updated, the WCAG 2.0 Guidelines should be implemented ("W3C HTML," 2009). Comparisons between WCAG 1.0 and WCAG 2.0 ("W3C Comparison of WCAG 1.0 Checkpoints to WCAG 2.0," 2009) are available. Time will be needed to transition for WCAG 1.0 to WCAG 2.0, since many web sites may need to be rewritten to comply with WCAG 2.0. Additionally, Web accessibility evaluation tools like ATRC Web Accessibility Checker (2009) are slow to be developed. Web-based applications need to address the following accessibility guidelines and apply an evaluation tool to the target Web pages:

- W3C Web Content Accessibility Guideline (WCAG 1.0) (“W3C," 2000) and (WCAG 2.0) (“WCAG 2.0 @ the Pickards,” 2009) compliance;

- Web Accessibility Initiative's Accessible Rich Internet Applications (WAIARIA) (W3C, 2006) compliance; and,

- Using the two guidelines (WCAG and WAI-ARIA) apply an accessibility tool ("W3C Complete List of Web Accessibility Evaluation Tools," 2009), like Fujitsu's Accessibility Inspector (2008) and ATRC Web Accessibility Checker tool (2009), to the web pages. 


\subsubsection{Web-based Applications' Evaluation Procedure}

Any applications identified as having a Web interface were evaluated against the Web-based Application Criteria, using the following procedure:

- Evaluate each Web application's HTML content and CSS content, for WCAG 1.0 and WCAG 2.0 compliance, using Fujitsu's Accessibility Inspector (2008) and the ATRC Web Accessibility Checker (2009);

- Visually check each item presented by the Fujitsu Accessibility Inspector and the ATRC Web Accessibility Checker (2009); and,

- Report findings.

\subsubsection{Web-based Collaborative Technology Evaluations}

Google Blog, Google Docs, Twitter, Microsoft SharePoint, and Group Systems' Think Tank were identified as representatives of web applications. Each application was evaluated for compliance to Web Content Accessibility Guidelines (WCAG) 1.0 ("W3C," 2000) and 2.0 ("W3C HTML," 2009). Even though WCAG 1.0 has been replaced by WCAG 2.0 Guidelines in 2008, both Guidelines were utilized for a complete analysis of accessibility. Table 6 specifies the priorities of WCAG 1.0, which are renamed levels of WCAG 2.0 in Table 7.

\subsubsection{Fujitsu's Web Accessibility Inspector - WCAG 1.0}

WCAG Guidelines are separated into three priority levels, which indicate the level of severity of the accessibility issue. The web content developer must implement solutions for the priority level 1 issues in order to provide accessibility to persons with 
disabilities ("W3C," 2000). The priority level 2 accessibility issues should be corrected by the web content developer, but are not required, in order to provide accessibility to persons with disabilities (“W3C," 2000). The web content developer may choose to or not to implement priority level 3 accessibility issues, to provide accessibility for persons with disabilities (“W3C," 2000). Fujitsu's Web Accessibility Inspector was used to evaluate Google Blog, Think Tank, Twitter, Google Docs and Microsoft SharePoint for WCAG 1.0 compliance (Table 6).

Table 6. Fujitsu's Web Accessibility Inspector Evaluation Results

\begin{tabular}{|c|c|c|c|}
\hline $\begin{array}{c}\text { Collaborative } \\
\text { Application }\end{array}$ & Priority 1 & Priority 2 & Priority 3 \\
\hline Google Blog & $\begin{array}{l}\text { Ten (10) - use of } \\
\text { special technology } \\
\text { or plug, embed all } \\
\text { information to be } \\
\text { keyboard accessible }\end{array}$ & $\begin{array}{l}\text { Eight (8) - small } \\
\text { text with small gaps } \\
\text { between lines, } \\
\text { describe the link } \\
\text { with text. }\end{array}$ & $\begin{array}{l}\text { One }(1)-\text { no } \\
\text { language is } \\
\text { specified. }\end{array}$ \\
\hline Google Docs & Zero (0) & $\begin{array}{l}\text { Two (2) - } \\
\text { DOCTYPE should } \\
\text { be specified for } \\
\text { HTML pages, } \\
\text { describe the link. }\end{array}$ & $\begin{array}{l}\text { One }(1)-\text { the } \\
\text { language attribute } \\
\text { is missing. }\end{array}$ \\
\hline $\begin{array}{l}\text { Microsoft } \\
\text { SharePoint }\end{array}$ & $\begin{array}{l}\text { Five (5) - no script } \\
\text { alternative to the } \\
\text { script parameter. }\end{array}$ & $\begin{array}{l}\text { Eight (8) - } \\
\text { DOCTYPE should } \\
\text { be specified for } \\
\text { HTML pages. }\end{array}$ & $\begin{array}{l}\text { Fifty-three (53) - } \\
\text { language attribute } \\
\text { is missing, tables } \\
\text { without a caption } \\
\text { for identification. }\end{array}$ \\
\hline Think Tank & $\begin{array}{l}\text { Three (3) - frameset } \\
\text { specified without } \\
\text { specifying "no } \\
\text { frameset." }\end{array}$ & $\begin{array}{l}\text { Three (3) - the } \\
\text { DOCTYPE should } \\
\text { be specified for } \\
\text { HTML pages. }\end{array}$ & $\begin{array}{l}\text { Two (2) - } \\
\text { language attribute } \\
\text { missing. }\end{array}$ \\
\hline Twitter & $\begin{array}{l}\text { Two (2) - no script } \\
\text { alternative. }\end{array}$ & $\begin{array}{l}\text { Six (6) - text size is } \\
\text { fixed by font size, } \\
\text { line spacing set to } \\
\text { fixed line height. }\end{array}$ & $\begin{array}{l}\text { Three (3) - } \\
\text { insufficient } \\
\text { contrast. }\end{array}$ \\
\hline
\end{tabular}




\subsubsection{Adaptive Technology Resource Center (ATRC) Web Accessibility Checker}

Since new accessibility guidelines (WCAG 2.0) were developed in December 2008, it was necessary to evaluate all web-based collaborative applications using this newest guideline. The primary benefits to using WCAG 2.0 Guidelines over the prior WCAG 1.0 Guidelines are: the descriptive nature of the identified accessibility issue makes it easy to understand and correct; the precise step-by-step test procedure is easy to follow and implement to identify accessibility issues; and, the precise solution (success criterion) for solving the identified accessibility issue.

The University of Toronto's Adaptive Technology Resource Center (ATRC) Web Accessibility Checker (2009) was the tool selected, due to its availability and ease of use, to evaluate Web Sites for WCAG 2.0 compliance. ATRC Web Accessibility Checker follows the success criterion procedures (a series of steps to verify accessibility), to determine if the HTML script and Style Sheets (CSS) meet the four criteria (perceivable, operable, understandable and robust) for accessible web pages. A success criterion (i.e. alternative text for images) is met when the success criterion procedure is verified against the actual web page. TRC Web Accessibility Checker was used to evaluate Google Blog, Think Tank, Twitter, Google Docs and Microsoft SharePoint for WCAG 2.0 compliance (Table 7). 
Table 7. (ATRC) Web Accessibility Checker Evaluation Results

\begin{tabular}{|l|l|l|l|}
\hline \multicolumn{1}{|c|}{ Application } & \multicolumn{1}{|c|}{ Level 1 } & \multicolumn{1}{c|}{ Level 2 } & \multicolumn{1}{c|}{ Level 3 } \\
\hline Google Blog & $\begin{array}{l}\text { Twenty-two (22) - } \\
\text { anchor contains no } \\
\text { text, document } \\
\text { language not } \\
\text { identified. }\end{array}$ & $\begin{array}{l}\text { One (1) - image } \\
\text { alternative text may } \\
\text { be too long. }\end{array}$ & $\begin{array}{l}\text { Five hundred and } \\
\text { forty (540) - } \\
\text { change of content } \\
\text { may occur without } \\
\text { user activation } \\
\text { image may } \\
\text { contain text with } \\
\text { poor contrast. }\end{array}$ \\
\hline Google Docs & $\begin{array}{l}\text { Two (2) - document } \\
\text { language is not } \\
\text { identified. }\end{array}$ & Zero (0) & $\begin{array}{l}\text { Sixteen (16) - } \\
\text { groups of links } \\
\text { with related } \\
\text { purpose is not } \\
\text { marked. }\end{array}$ \\
\hline Microsoft SharePoint & $\begin{array}{l}\text { Seventy-two (72) - } \\
\text { input element has } \\
\text { alt attribute, input } \\
\text { element type text } \\
\text { missing associated } \\
\text { label. }\end{array}$ & $\begin{array}{l}\text { Two (2) issues - } \\
\text { select element's } \\
\text { label is not } \\
\text { positioned close to } \\
\text { control. }\end{array}$ & $\begin{array}{l}\text { Three hundred } \\
\text { ninety-six (396) - } \\
\text { input element } \\
\text { label, type of } \\
\text { "text," not } \\
\text { positioned to close } \\
\text { to the control. }\end{array}$ \\
\hline Think Tank & $\begin{array}{l}\text { Four (4) - frame } \\
\text { missing title } \\
\text { attribute. }\end{array}$ & $\begin{array}{l}\text { Zero (0) } \\
\text { Five (5) - input } \\
\text { index. }\end{array}$ & $\begin{array}{l}\text { Ten (10) - } \\
\text { repeated blocks of } \\
\text { content may not } \\
\text { appear in the same } \\
\text { frame within the } \\
\text { same frame set. }\end{array}$ \\
\hline Twitter & Zero (0) & $\begin{array}{l}\text { Eighty (80) - tab } \\
\text { order may not } \\
\text { follow logical } \\
\text { order, form } \\
\text { submission errors. }\end{array}$ \\
\hline
\end{tabular}




\subsubsection{Comparing WCAG 1.0 to WCAG 2.0}

WCAG has implemented a rating system for determining the quality of the Web pages' accessibility. WCAG utilizes a rating system of 'A', 'AA', or 'AAA.' WCAG 1.0 utilizes priorities from 1 to 3 to identify ratings, while WCAG 2.0 utilizes levels from 1 to 3 to identify ratings ("WCAG 2.0 @ the Pickards," 2009). The fundamental reason for the change from priority to level was to clarify that all accessibility issues were important and that no one issue should be a priority over any other accessibility issue ("WCAG 2.0 @ the Pickards," 2009).

For WCAG 1.0, implementing all of the priority 1 checkpoints qualifies the Web site as a rating of ' $A$ '. Implementing all of priority 1 and 2 checkpoints qualifies the Web site as a rating of 'AA.' Finally, implementing all of the priority 1 and 2 and 3 checkpoints qualifies the Web site as a rating of 'AAA.'

For WCAG 2.0, implementing all of the level 1-success criterions qualifies the Web site as a rating of ' $A$ '. Implementing all of the level 1 and 2 success criterions qualifies the Web site as a rating of 'AA.' Finally, implementing all of the level 1 and 2 and 3 success criterions qualifies the Web site as a rating of 'AAA.' Figure 2 provides a breakdown of the WCAG 2.0 Guideline and each guideline's relationship to the 'A' rating scheme. The four principles of perceivable, operable, understandable and robust are used to group the WCAG 2.0 Guidelines. Grouping the WCAG 2.0 Guidelines into general principles provides understandability for the persons implementing them. Each WCAG 2.0 Guideline has a set of sub-guidelines (i.e. 3.1.2 - rating 'AA') and if implemented for a web site aids in determining the rating. 


\begin{tabular}{|c|c|c|c|c|}
\hline Principles & Guidelines & Level A & Level AA & Level AAA \\
\hline \multirow[t]{4}{*}{ 1. Perceivable } & 1.1 Text Alternatives & 1.1 .1 & & \\
\hline & 1.2 Time-based Media & $1.2 .1-1.2 .3$ & $1.2 .4-1.2 .5$ & $1.2 .6-1.2 .9$ \\
\hline & 1.3 Adaptable & $1.3 .1-1.3 .3$ & & \\
\hline & 1.4 Distinguishable & $1.4 .1-1.4 .2$ & $1.4 .3-1.4 .5$ & $1.4 .6-1.4 .9$ \\
\hline \multirow[t]{4}{*}{ 2. Operable } & 2.1 Keyboard Accessible & $2.1 .1-2.1 .2$ & & 2.1 .3 \\
\hline & 2.2 Enough Time & $2.2 .1-2.2 .2$ & & $2.2 .3-2.2 .5$ \\
\hline & 2.3 Seizures & 2.3 .1 & & 2.3 .2 \\
\hline & 2.4 Navigable & $2.4 .1-2.4 .4$ & $2.4 .5-2.4 .7$ & $2.4 .8-2.4 .10$ \\
\hline \multirow[t]{3}{*}{ 3. Understandable } & 3.1 Readable & 3.1 .1 & 3.1 .2 & $3.1 .3-3.1 .6$ \\
\hline & 3.2 Predictable & $3.2 .1-3.2 .2$ & $3.2 .3-3.2 .4$ & 3.2 .5 \\
\hline & 3.3 Input Assistance & 3.3.1-3.3.2 & 3.3.3-3.3.4 & 3.3.5-3.3.6 \\
\hline 4. Robust & 4.1 Compatible & $4.1 .1-4.1 .2$ & & \\
\hline
\end{tabular}

Figure 2 WCAG 2.0 and its relationship to the "A' rating scheme ("WCAG 2.0 @ the Pickards," 2009)

Comparing WCAG 1.0 priorities to WCAG 2.0 levels is not practical, since the priorities and levels are not the same. Many of the WCAG 1.0 priorities have moved to different levels and in some cases have split from one priority to many levels. The best comparison at this point is in comparing details about each of the WCAG 1.0

Checkpoints against the WCAG 2.0 Success Criterion and the number of issues identified.

The issues presented by the WCAG 1.0 evaluation seemed minor in comparison to the issues presented by the WCAG 2.0 evaluation. The issues presented by the WCAG 1.0 evaluation included scripting issues, but the concern was in providing alternatives to the scripts. Additionally, the detail of the scripting issues is not as specific as the scripting issues identified under WCAG 2.0. The focus with the WCAG 1.0 evaluation seemed to focus more on structure. For example, frames without frame names, missing titles and 
missing alternative text. The Fujitsu's Web Accessibility Inspector (2008) WCAG 1.0 evaluation identified the following common issues among all of the applications evaluated:

- Special technology (JavaScript) and mouse events causing keyboard access issues;

- Poor contrast and small text causing low-vision access issues;

- Lack of table captions causing access and search issues by blind persons; and,

- Missing language parameters causing enunciation issues for screen readers.

The issues presented by the WCAG 2.0 evaluation seemed to impact the entire interface (forms, controls, text contrast) and unhandled events (delete without recovery, loading a new web page may cause a new window to open, document may require breadcrumb trail) which may indicate the need to include WAI-ARIA metadata to handle the events and make the interface accessible. Scripts and events related to scripts seem to be a major area of concern for WCAG 2.0 evaluations, since rich internet applications such as collaborative applications are including components (text boxes, trees, command buttons, etc.) and dynamic content (events). The focus with the WCAG 2.0 evaluation focused on goals like perceivable, operable, understandable and robustness. 
The ATRC Web Accessibility Checker (2009) WCAG 2.0 evaluations identified the following common issues among all of the applications evaluated:

- Issues related to interface control and input;

- Issues related to clustering;

- Issues related to form interaction; and,

- Issues related to objects and text contrasts.

The WCAG 1.0 and 2.0 evaluations (Table 8), using both Fujitsu's Web Accessibility Inspector (2008) and ATRC Web Accessibility Checker (2009), reported all applications verified for accessibility contained accessibility issues rendering all inaccessible to persons with disabilities. The number of accessibility issues discovered during the WCAG 2.0 evaluation was greater than the number of accessibility issues discovered during the WCAG 1.0 evaluation. Again, it should be stated that there might be many false positives that really do not prevent access to the web content. Only through detailed user review can the actual accessibility be determined. This difference can be attributes to two possibilities: the web pages have not been upgraded to comply with WCAG 2.0; or, the criteria for evaluating WCAG 2.0 have become more detailed. 
Table 8. Web-based Accessibility Evaluations Summary

\begin{tabular}{|c|c|c|}
\hline Collaborative Application & $\begin{array}{l}\text { WCAG 1.0- } \\
\text { Fujitsu's Web } \\
\text { Accessibility } \\
\text { Checker }\end{array}$ & $\begin{array}{l}\text { WCAG 2.0- } \\
\text { ATRC Web } \\
\text { Accessibility } \\
\text { Checker }\end{array}$ \\
\hline $\begin{array}{l}\text { Google Blog } \\
\text { http://googleblog.blogspot.com }\end{array}$ & $\begin{array}{l}19 \text { issues }-10 \\
\text { priority } 1 ; 8 \text { priority } \\
2 ; \text { and, } 1 \text { priority } 3 .\end{array}$ & $\begin{array}{l}563 \text { issues }-22 \\
\text { level } 1 ; 1 \text { level } 2 \text {; } \\
\text { and, } 540 \text { level } 3 .\end{array}$ \\
\hline Google Docs http://docs.google.com & $\begin{array}{l}3 \text { issues }-0 \text { priority } \\
1 ; 2 \text { priority } 2 ; \text { and, } \\
1 \text { priority } 3 .\end{array}$ & $\begin{array}{l}18 \text { issues }-2 \text { level } \\
1 ; 0 \text { level } 2 \text {; and, } \\
16 \text { level } 3 \text {. }\end{array}$ \\
\hline $\begin{array}{l}\text { Microsoft SharePoint } \\
\text { http://www.microsoft.com/Sharepoint/defa } \\
\text { ult.mspx }\end{array}$ & $\begin{array}{l}66 \text { issues }-5 \\
\text { priority } 1 ; 8 \text { priority } \\
2 ; \text { and, } 53 \text { priority } \\
3 .\end{array}$ & $\begin{array}{l}470 \text { issues }-72 \\
\text { level } 1 ; 2 \text { level } 2 \text {; } \\
\text { and, } 396 \text { level } 3 \text {. }\end{array}$ \\
\hline $\begin{array}{l}\text { Group Systems Think Tank } \\
\text { http://www.groupsystems.com/thinktank }\end{array}$ & $\begin{array}{l}8 \text { issues }-3 \text { priority } \\
1 ; 3 \text { priority } 2 ; \text { and, } \\
2 \text { priority } 3 \text {. }\end{array}$ & $\begin{array}{l}85 \text { issues }-5 \text { level } \\
2 ; 0 \text { level } 2 \text {; and, } \\
80 \text { level } 3 .\end{array}$ \\
\hline $\begin{array}{l}\text { Twitter } \\
\text { http://twitter.com } \\
\end{array}$ & $\begin{array}{l}11 \text { issues }-2 \\
\text { priority } 1 ; 6 \text { priority } \\
2 \text {; and } 3 \text { priority } 3 .\end{array}$ & $\begin{array}{l}14 \text { issues }-4 \\
\text { known; } 0 \text { likely; } \\
\text { and, } 10 \text { potential. }\end{array}$ \\
\hline
\end{tabular}

3.5 Manual Accessibility and Usability Evaluations

The next sub-sections will discuss the criteria used to evaluate Windows, console, and Web applications; the procedure used to evaluate Microsoft Windows, console (VT100, text-based terminal), and Web applications; and, the evaluations of Microsoft Outlook, Microsoft SharePoint, America Online Instant Messenger (AIM), Google Blog, Twitter, Google Docs, and Group Systems' Think Tank. 


\subsubsection{Windows, Console and Web Applications' Evaluation Criteria}

In addition to the Section 508 (2013), 1194-21 Software Applications and Operating Systems Guideline, Schoeberlein and Wang (2009b) identified factors or criteria that should be considered when evaluating the accessibility of these types of applications:

- Support of accessible interface prototype usage (Hampel et al., 1999; Takagi et al., 2000; Watanabe et al., 2007; Winberg et al., 2004).

- Navigating hierarchies (Watanabe et al., 2007; Waters et al., 2005).

- Single lists (Watanabe et al., 2007; Waters et al., 2005) of choices.

- Integrated auditory input.

- Text-to-Braille (integrated) (Hampel et al., 1999).

- Screen reader (Watters et al., 2005) support. 


\subsubsection{Windows, Console and Web Applications' Evaluation Procedure}

The applications Microsoft Outlook, Microsoft SharePoint, America Online Instant Messenger (AIM), Google Blog, Twitter, Google Docs, and Group Systems' Think Tank were evaluated against the Windows, Console and Web Application's Evaluation Criteria (Schoeberlein \& Wang, 2009b), using the following procedure:

1. Search the Internet, to determine the availability of an API for the evaluated application, to determine if accessible interface prototypes may be built. This would allow the expansion of the interface to add accessibility features (text-toBraille, speech recognition) to the applications, or correct the interaction between the screen reader and a specific control on the interface;

2. Review the application's documentation for the support of integrated audio input support, screen reader support, and integrated text-to-Braille support, to determine if any built-in accessibility feature are shipped with the application;

3. Execute the application, using only the keyboard interface and the JAWS Screen Reader, and determine the accessibility and usability of the interface by tabbing from control-to-control and parsing (tabbing with the keyboard) any hierarchies or lists. Specific tasks normally accomplished with the application are followed, using only the keyboard to simulate the interface activity of persons with disabilities; and,

4. Report findings. 


\subsubsection{API Availability}

Seven applications including Microsoft Outlook, Microsoft SharePoint, America Online Instant Messenger (AIM), Google Blog, Twitter, Google Docs, and Group Systems' Think Tank were evaluated for API availability. All of the applications, except Group Systems' Think Tank, provide an API to allow Developers to build custom interfaces to each application. The availability of an API is required to the development of accessible interface prototypes that can be utilized in test environments to solve accessibility and usability issues.

\subsubsection{Documentation Review}

A review of the applications' documentation for integrated audio input support, screen reader support, and integrated text-to-Braille support was conducted, to determine if any built-in accessibility features are shipped with the applications.

Limited audio input is available only with AOL AIM and Microsoft Outlook. For AOL AIM, audio input is available in the form of voice Chat. Voice Chat has to be setup on the person's computer and then they can invite others to a voice Chat session. For Microsoft Outlook, audio input is available in the form of dictation. Spoken text is converted to electronic text, similar to typing on the keyboard. Setup of both voice Chat and dictation may be difficult for some persons with disabilities.

If a Web solution is implemented for an application, then several levels have to interact in unison (server application, web browser and screen reader). The server application has to implement WAI-ARIA metadata to communicate to screen readers the interface's components and events. The web browser has to recognize the WAI-ARIA 
metadata, so it is syntactically correct and not ignored or rejected. The screen reader has to recognize the WAI-ARIA metadata in order to identify components and respond to events. Simply having a screen reader interact with a rich Internet application will not work due to missed events and the inability to access some components on the interface (Mikovec et al., 2009; Buzzi et al., 2008). It is important to review each application to determine if the screen reader can provide accessibility to the application's features. If the screen reader does not handle components or events properly, WAI-ARIA metadata may need to be implemented

Text-to-Braille built into applications may provide an alternative output medium that persons who are blind can access and read anytime. Text-to-Braille is not available with the shipment of any of the applications under review.

\subsubsection{Execute the Application}

Each application underwent expert review/inspection in order to determine if a collaborative application was accessible and usable. The tests were conducted using only the keyboard interface and the JAWS Screen Reader with the computer turned on. The use of a mouse device was eliminated, to simulate the environment of persons with some disabilities (i.e. persons who are blind, or visually impaired) who do not use the mouse to interact with the computer. 
The evaluation procedure of the computer interactions included the following steps:

1. Evaluate the application by turning on the computer and running the application. This step is utilized to determine general access and use of the application. Since persons who are blind do not utilize a mouse for input, only the keyboard was utilized. Using only the keyboard and the JAWS Screen Reader inspect the application's interface, and determine the accessibility and usability of the interface by:

- Tabbing from control-to-control and parsing (tabbing with the keyboard) any hierarchies or lists; and,

- Execute tasks normally accomplished with the application. For example, with a Word Document, typical tasks are evaluated such as opening, editing, saving and closing a document.

2. Report the findings of the review/examination.

\subsubsection{Microsoft Outlook Evaluation}

Microsoft Outlook (2008) is a part of the Microsoft Office Suite. Although, it is most often used as an email application, it also includes support for other group activities, such as: Calendar, Task Manager and Contact Manager. Outlook can be used either as a stand-alone application, or operates in conjunction with Microsoft Exchange Server and Microsoft Office SharePoint server, to provide enhanced functions for group members, such as group calendars for meeting scheduling, task monitoring, and public folders exchanges, etc. 
On reviewing Microsoft Outlook for accessibility the following accessibility issues were identified:

- Profile popup was not recognized by the JAWS Screen Reader;

- Deep hierarchy navigation, using the keyboard interface was difficult; and,

- No text-to-Braille support as an alternative output mode.

On loading Microsoft Outlook a user profile (Figure 3) displays pop-up message box requesting the user name and password to gain access to the email interface. Notification of popup message boxes should be presented in an auditory form, or the JAWS Screen Reader should recognize this event and present an audio output message to allow responses by the end users. In this case the JAWS Screen Reader did not recognize the login message box.

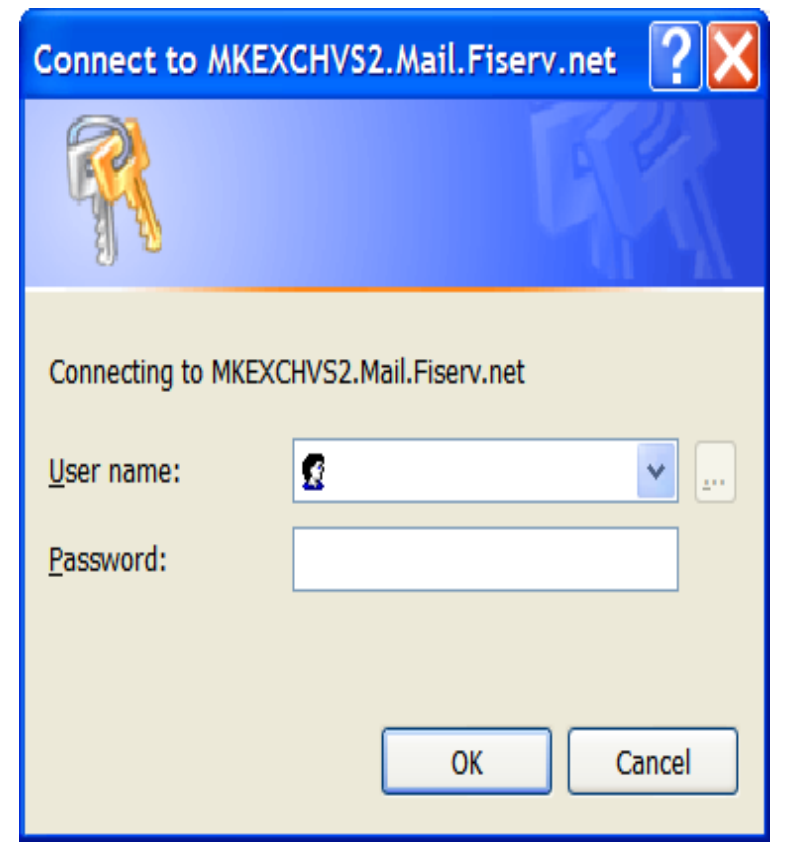

Figure 3 Microsoft Outlook Login Profile 
Microsoft Outlook's interface typically consists of three main panels for listing and viewing email messages (Figure 4). The left panel consists of a hierarchy of folders providing structure and organization to the email messages. The center panel consists of the email message subjects for the selected folder in the left panel. The right panel contains the detail of the email message for the selected message subject in the center panel.

The hierarchy folders and panels of Microsoft Outlook are difficult to transverse with just keyboard navigation. When moving through different levels of a hierarchy, orientation and place are compromised. Deep (depth) hierarchies are difficult to navigate, due to their complexity.

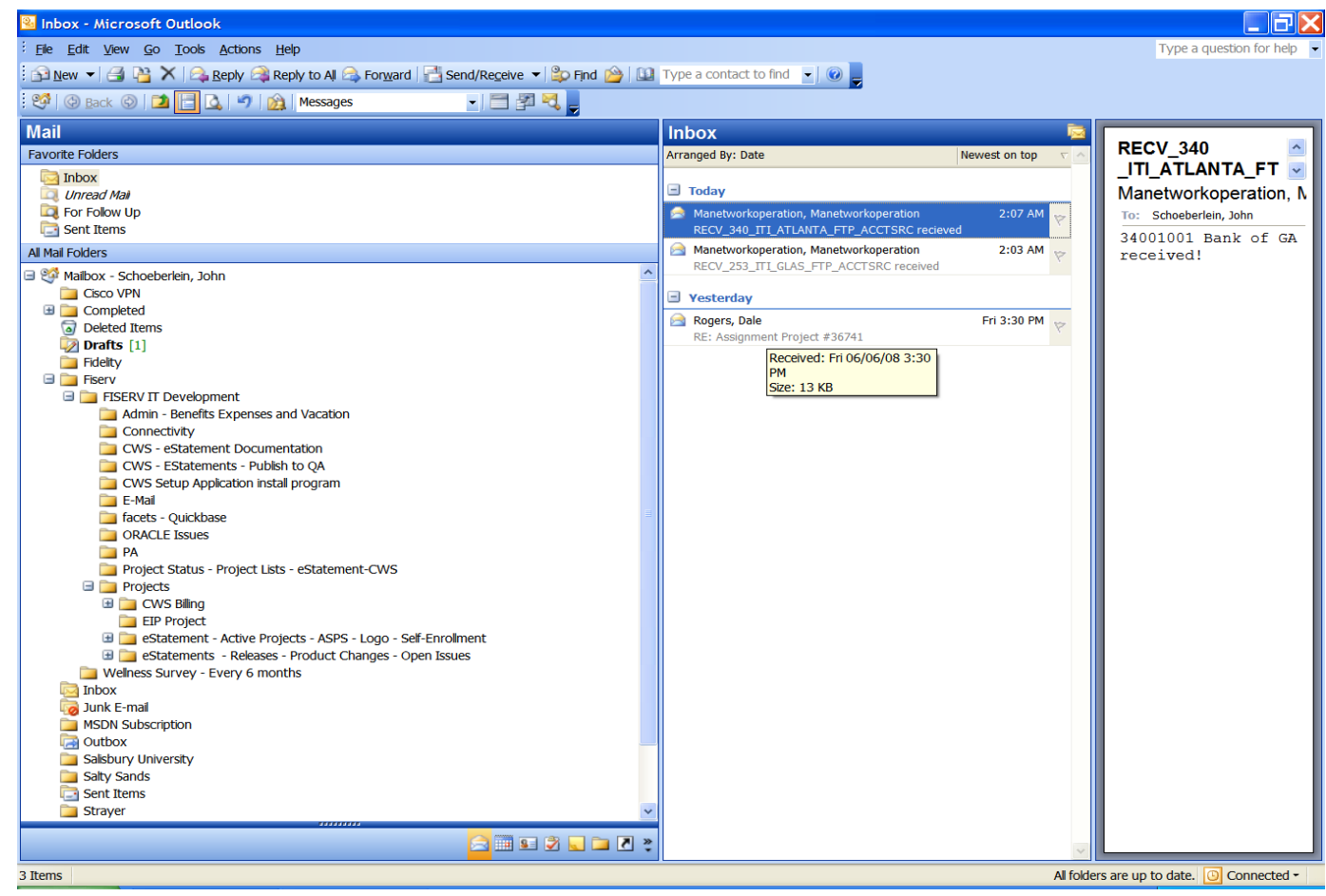

Figure 4 Microsoft Outlook's Folders and Panels 


\subsubsection{America Online Instant Messenger (AIM®) Evaluation}

With about 89 million users worldwide (“AOL Company Overview," 2009), AOL provides several versions of instant messaging software that support different platforms. AIM (AIM, 2008) is a Windows collaborative application for presenting, creating and reading Chat messages. Through its buddy list window, users can see whether their buddies are online or available for Chat.

Another version of AIM ${ }^{\circledR}$, AIM Express, now provides a Web interface that allows its members to Chat in a browser without having to download the application. On reviewing AIM for accessibility the following accessibility issues were identified:

- Hierarchy navigation, using the keyboard interface was difficult; and,

- No text-to-Braille optional output support.

The hierarchy nature (Buddies List) of AIM is difficult to transverse. The depth of the Buddies List is not an issue, but navigating with just the keyboard interface was difficult. The right-arrow key press opens the folder and exposes child folders in the hierarchy or child folders containing a list of buddies (Figure 5). The left-arrow key press closes the folder hiding the child folders in the hierarchy. Navigation up and down the hierarchy is accomplished by pressing the up-arrow and the down-arrow. 


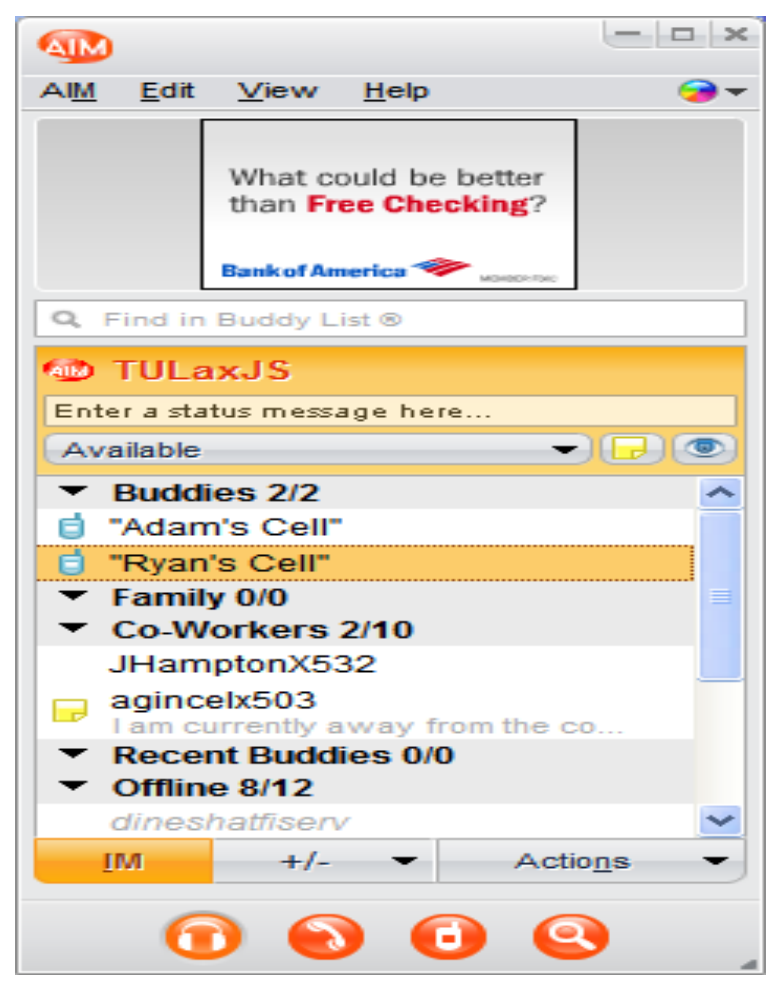

Figure 5 AOL AIM Buddy List

\subsubsection{Twitter Evaluation}

Twitter (2009) is a Web collaborative application for mini-blogging, which prompts the question: "What are you doing?" Persons utilize this collaborative application to keep in-touch with organizations, such as The New York Times, with celebrities, with group members in their Company, and with friends and family.

On reviewing Twitter for accessibility the following accessibility issues were identified:

- No integrated auditory input;

- No integrated text-to-Braille support; and,

- Inability to access the Twitter Menu using the keyboard. 
The lack of auditory input prevents some persons with disabilities no alternative for data input. Integrated text-to-Braille, similar to Takagi (2000) for text-to-speech and Hampel (1999) for text-to-Braille, could be included to allow for alternative delivery channels for persons with disabilities. Additionally, the Twitter Menu options (Figure 6) (Home, Profile, Find People, Settings, Help, and Sign-out) are inaccessible with the keyboard. Tabbing with the keyboard's tab key, from control-to-control, moves the cursor from the Web browser's menu into the body of the Twitter content. Unfortunately, the Twitter Menu, located within the body of the Twitter content, is not accessible. Basically, the Twitter Menu becomes part of the body of the interface and is accessible only with the mouse, which is not utilized by some persons with disabilities.

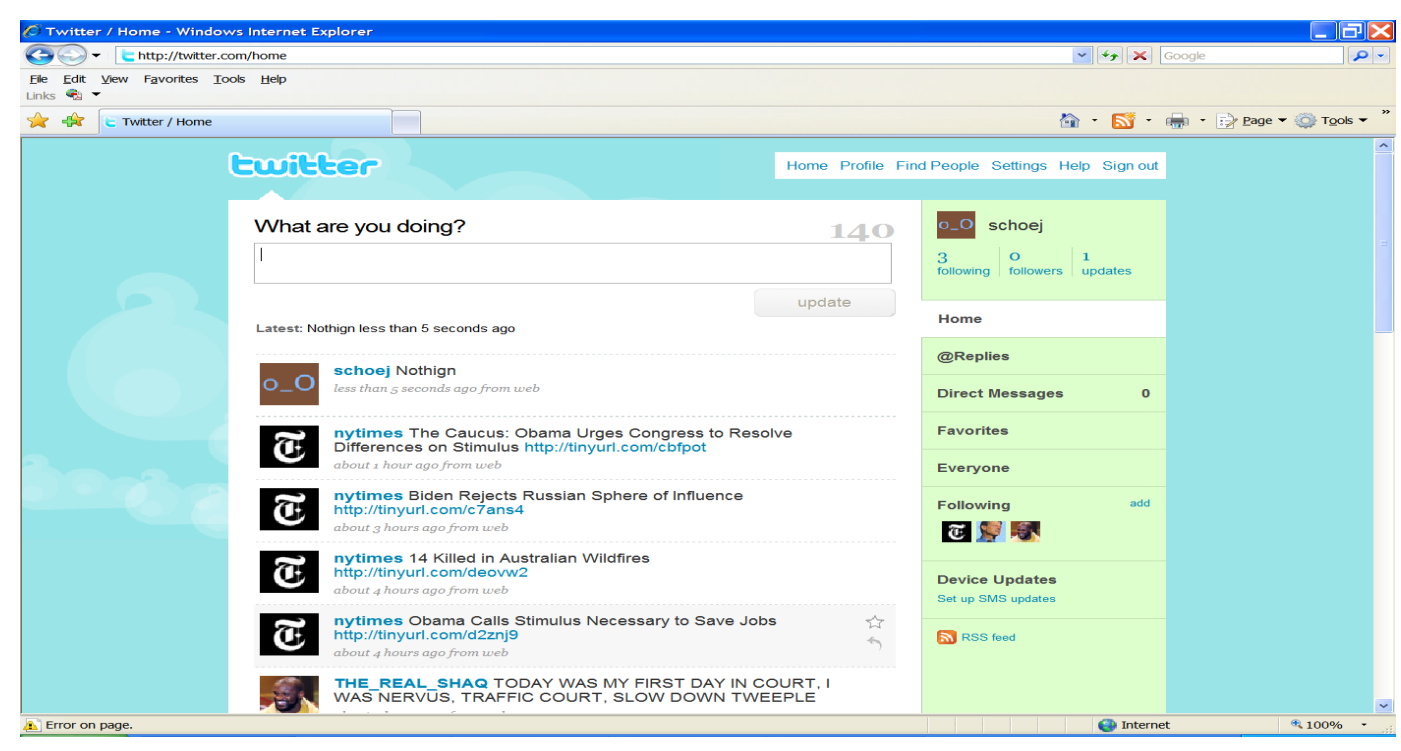

Figure 6 Twitter Interface and Menu (Home, Profile, Find People, Settings, Sign-out and Help) 


\subsubsection{Google Blog Evaluation}

Google Blog (2008) is a Web collaborative application that allows persons to share their thoughts about current events or anything they'd like to discuss. The shared thoughts are called blogs (web logs). Owners of a blog, bloggers, are able to form or develop a community with people with similar interest, to receive feedback from their readers. This application also allows group blogging, which enables a team of bloggers to contribute to a single blog, thus enables more close collaboration among members. The following accessibility issues were identified the following accessibility issues were identified:

- Blog list was difficult to navigate with the keyboard;

- No integrated auditory input (alternative input mode); and,

- No integrated text-to-Braille (alternative output mode).

Google Blog's interface consists of a main panel and a right panel containing content on a topic and a blog anchor to access the blog (Figure 7).

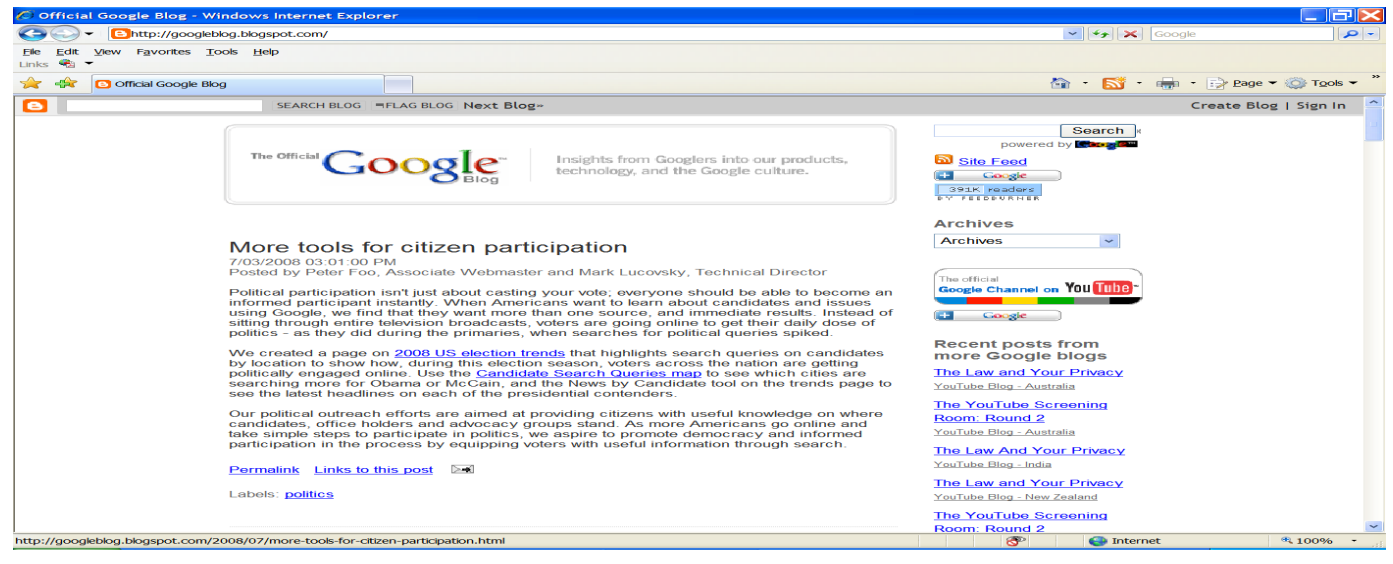

Figure 7 Google Blog Interface 
Navigation up and down the content to access the various blogs is accomplished by pressing the tab key or the alt-tab keys. The list of Google Blogs is difficult and tedious to transverse. To view the detail of a blog topic, select a specific anchor (Google Mac Blog, Figure 8, and Figure 9).

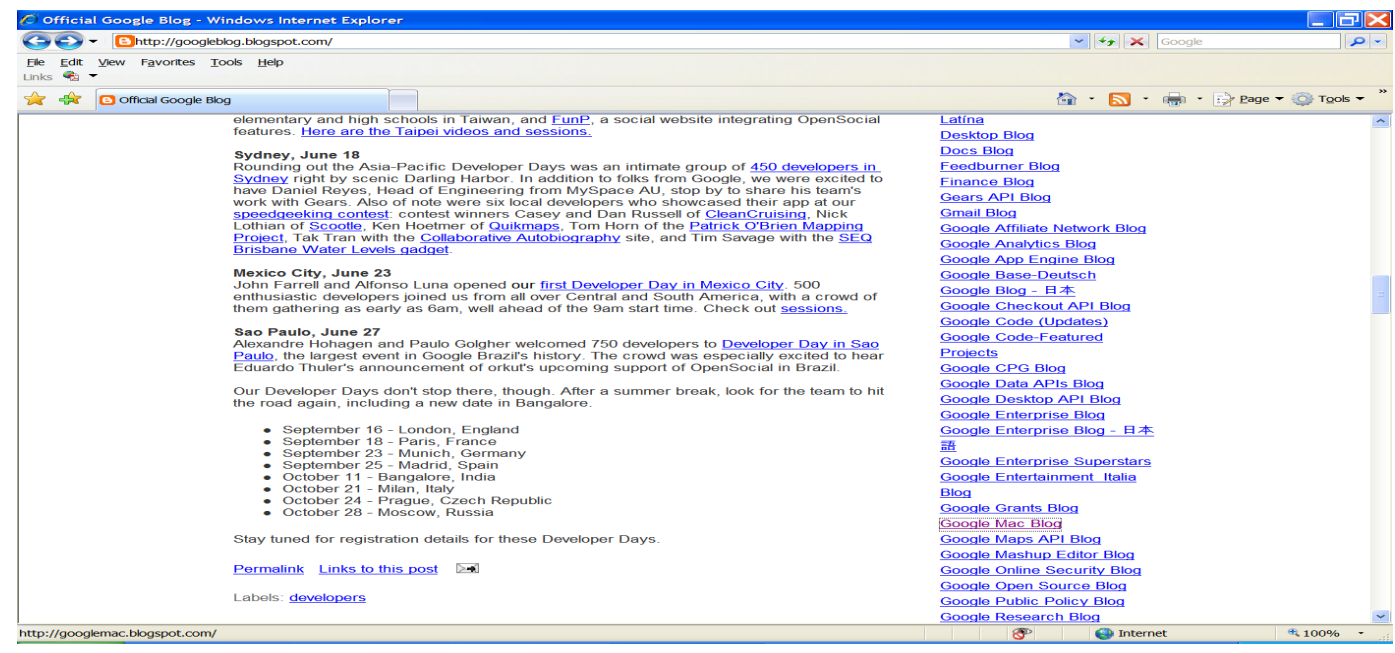

Figure 8 Google Mac Blog selected

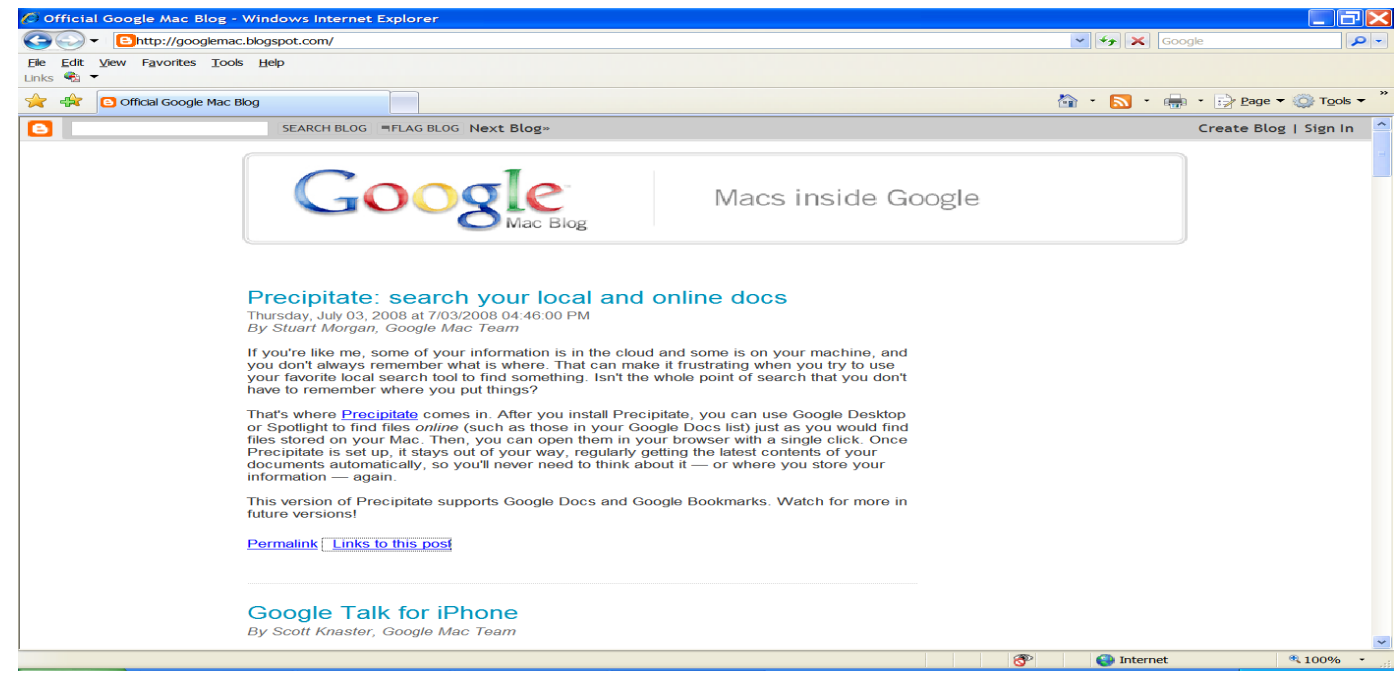

Figure 9 Google Mac Blog displayed 
Once the blog topic is selected, the specific blogs within the blog topic can be transverse by pressing the tab key and the alt-tab keys. A specific blog, "Mac inside Google" (Figure 10), can be blogged by selecting the "reply" anchor.

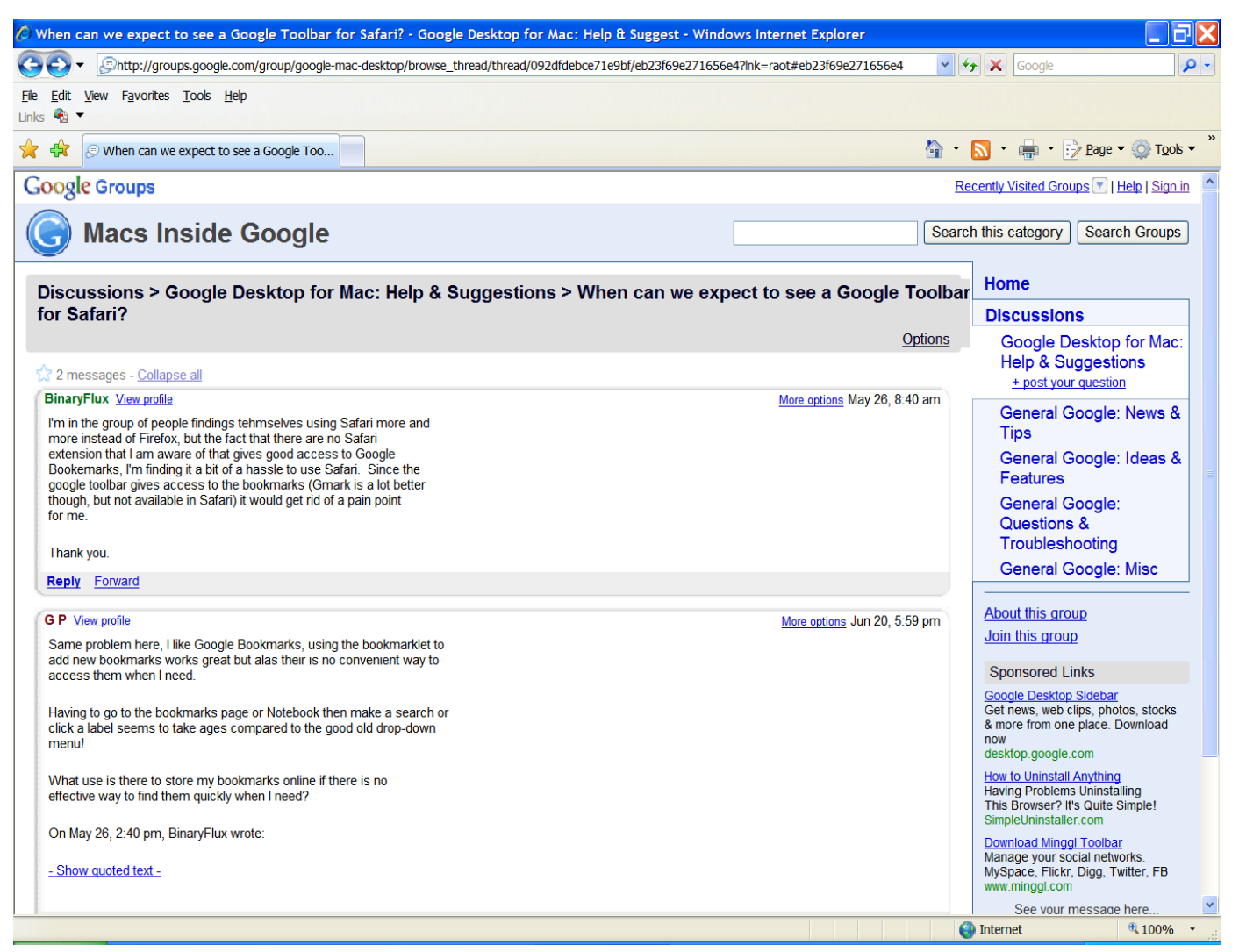

Figure 10 Google Mac Blog reply

Returning back to the Google Blog Interface (Figure 7), from a specific blog, was difficult. Some sort of technique should be included to go "back-to-top," to allow access to the Google Blog Interface (Figure 7). The lack of integrated auditory input prevents some persons with disabilities the ability to interact with the Google Blog interface. 


\subsubsection{Google Docs Evaluation}

Google Docs (2009) is a Web collaborative application that allows collaborators shared access to their documents, spreadsheets and presentations online. Google Docs allows document sharing by attaching email addresses to the document. This concept is the reverse of sending a document to many email addresses. In the Google Docs example, only one copy of the document exists for group collaboration. In the later example, multiple copies of a document exist at each email address, thus complicating group collaboration.

The following accessibility issues were identified:

- Inability to navigate to the document editing menu using the keyboard;

- No integrated auditory input (alternative input mode); and,

- No integrated text-to-Braille support (alternative output mode).

Google Docs contains two sets of menus (Figure 11), one set is the browser menu (File, Edit, View, etc.), and the second set is the document editing menus (File, Edit, View, Insert, etc.). 


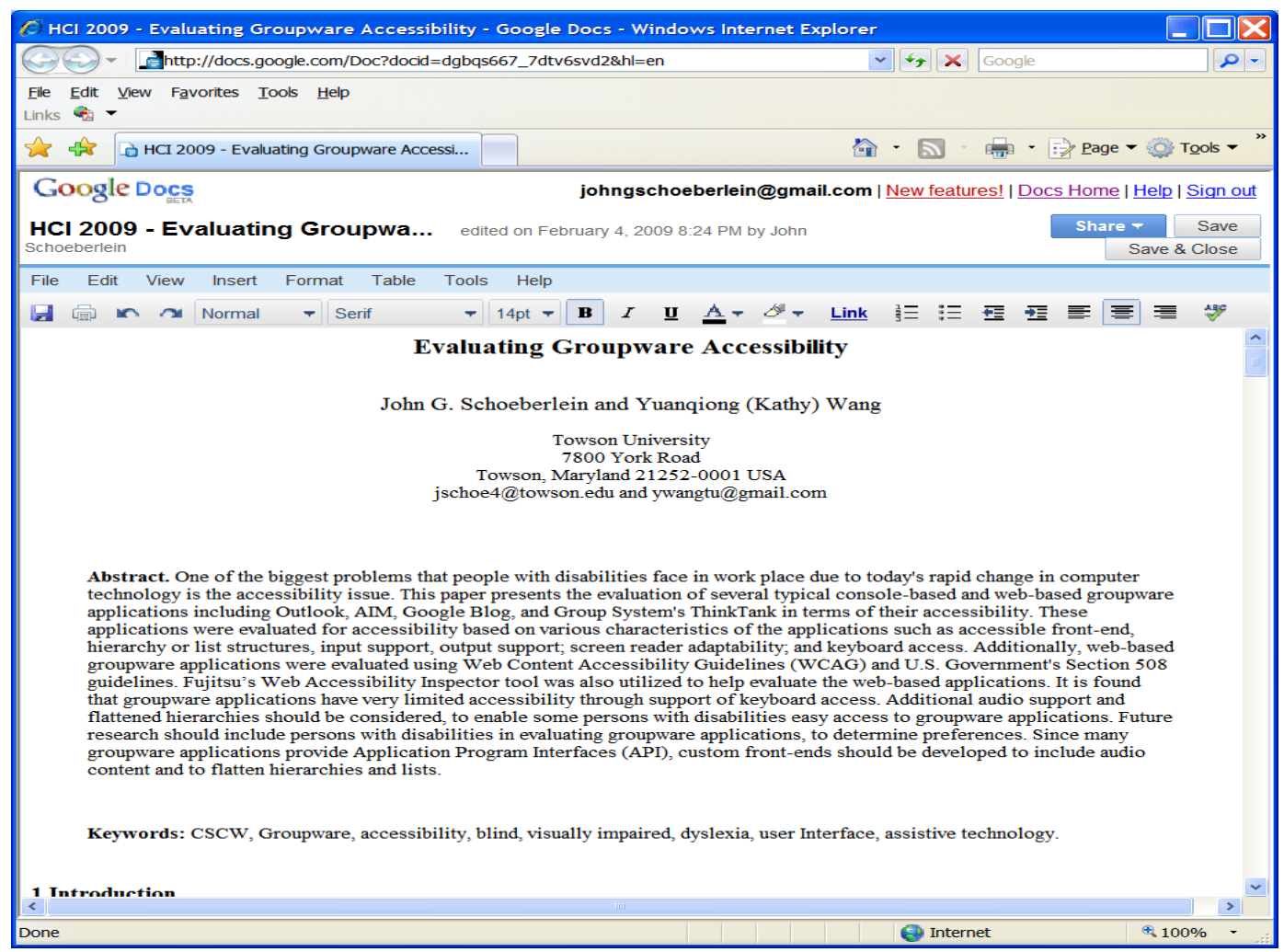

Figure 11 Google Docs Duel Menus

Keyboard access is limited to the browser menus; the document menus are not accessible using the keyboard. Since the keyboard access to the editing menus is not available, Google Docs is not accessible for persons with disabilities. The lack of integrated (built-in) auditory input prevents some persons with disabilities no alternative for data input.

\subsubsection{Group Systems' Think Tank Evaluation}

Group Systems' Think Tank (2008) is a Web-based groupware application for brainstorming, group decision-making and collaboration. It provides distributed or colocated groups with functions such as brainstorming, organizing ideas, prioritizing, voting, consensus building and documenting group knowledge. 
The following accessibility issues were identified:

- Profile popup was not recognized by the integrated DeskBot Genie ("Bell Craft DeskBot," 2008);

- Hierarchy navigation was difficult using the keyboard interface;

- No integrated auditory input (alternative input mode);

- No text-to-Braille support as an alternative output mode; and,

- Out-of-sync text-to-speech audio output by the integrated Genie.

On loading Think Tank, a user profile (Figure 12) displays requesting the user name and password to gain access to the group decision-making interface. The DeskBot Genie did not recognize the Pop-up message box.

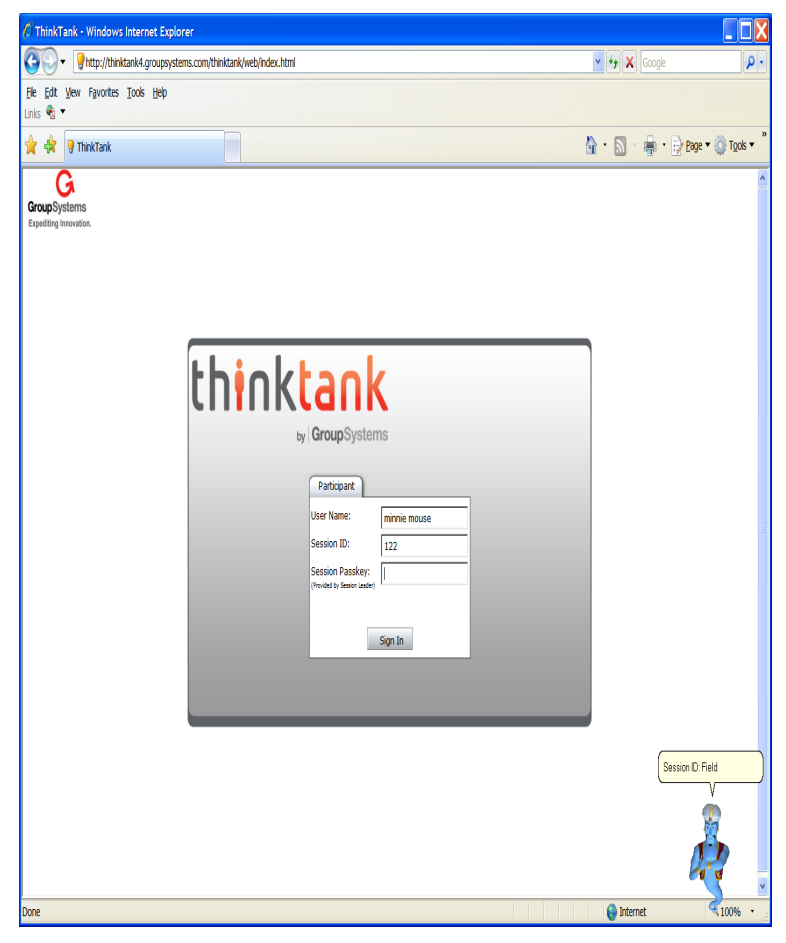

Figure 12 Think Tank - Login profile 
Navigating the hierarchy folder structure (Figure 13 - Agenda, Categories, Ideas, and Comments) is usually accomplished by using a mouse to select an idea category folder with the left-mouse button click. The keyboard can be used to navigate the hierarchy by pressing the up-arrow and the down-arrow. The enter key is pressed to open the category folder and exposes a list of ideas in a central panel. This multiple tiered hierarchy was difficult to navigate due to the complexity of orientation of such a structure. There are several controls on the web page (i.e. command buttons for tasks, including: brainstorm, organize, edit, delete, etc.) that have to be navigated in order to set the focus on the desired control. This navigation further complicates the interface.

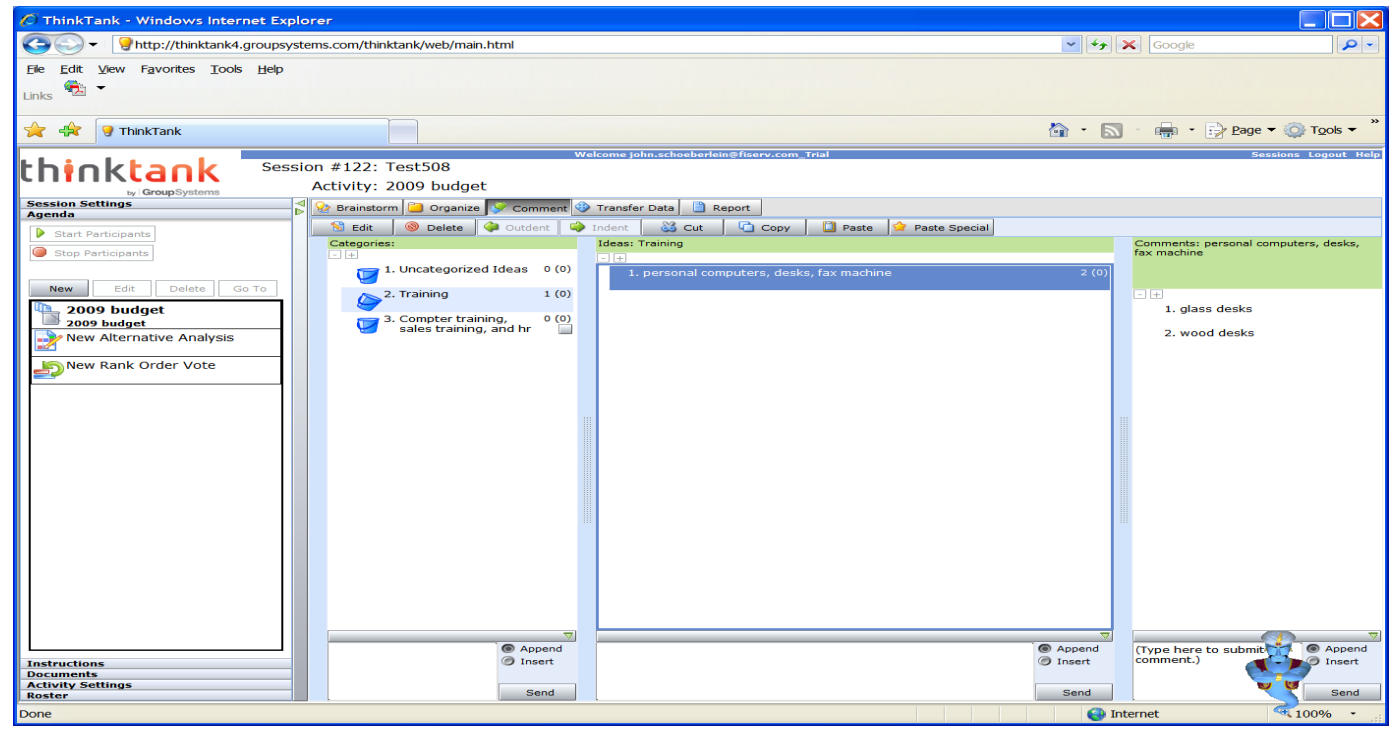

Figure 13 Think Tanks - Agenda, Categories, Ideas, and Comments

The screen reading program adapted by Think Tank, DeskBot Genie (Figure 13 Bottom right) ("Bell Craft DeskBot," 2008), can become out-of-sync in terms of audio output with the currently selected item, causing disorientation. Often, the DeskBot Genie will repeat the same content over-and-over again, which may cause confusion and disorientation. The DeskBot Genie is a stand-alone application downloaded to the client's 
machine that reads and converts to speech any text place on the Microsoft Clipboard Object. The developers of Think Tank rely on the DeskBot Genie to provide text-tospeech capabilities, to avoid the use of a screen reader, like JAWS.

\subsubsection{Microsoft SharePoint Evaluation}

Microsoft SharePoint (2009) is a Web collaborative server application that allows collaborators shared access to their documents and spreadsheets online. Microsoft SharePoint has additional features such as; Wiki, group calendar, group newsgroup, and blogs. The document sharing enlists Microsoft Word and Microsoft Excel to display and edit the contents of a document or spreadsheet. The document or spreadsheet is exclusively locked by the editor and is released for other collaborators to display or edit once the document is saved to the Microsoft SharePoint Server.

The following accessibility issues were identified:

- No integrated auditory input (alternative input mode); and,

- No integrated text-to-Braille support (alternative output mode).

\subsection{Comparing Collaborative Applications}

The selected collaborative applications were then compared against each other, to determine if any accessibility and usability patterns existed.

\subsubsection{Accessible Collaborative Interface Prototypes}

The Microsoft Outlook Object Library (2008); the AIM Object Library (2008); the Microsoft SharePoint Services Software Development Kit ("Microsoft SharePoint Services 3.0," 2009); the Google Blogger Data API (2008); the Twitter Object Library ("Twitter API," 2009); and, the Google Documents List Data API (2009) can be 
downloaded. Google Documents List Data API allows documents to be uploaded to the Google Documents database for collaboration. However, the Google Docs List Data API does not support collaborative editing of the document; you have to use the Google Docs Web Site for collaboration. Think Tank does not provide an API for creating an accessible collaborative interface prototype to encapsulate the complexity of the graphical interface.

\subsubsection{Hierarchies}

All applications investigated utilize hierarchies as their major approach when it comes to organizing information. Microsoft Outlook consists of a hierarchy of folders and sub-folders containing email messages; Microsoft SharePoint consists of a hierarchy of shared folders or document libraries containing shared documents. AOL AIM consists of a hierarchy of buddy lists containing buddies from various groups you can engage in a Chat session; Google Blog consists of a hierarchy of blog lists containing blog posts you can access to engage in a discussion; Google Docs consists of a hierarchy of documents you can edit; Think Tank's hierarchy consists of categories, ideas and comments, and Twitter is not hierarchical, but consists of a list of recent messages or mini blogs you can access to reply to the messages or continue the mini blog.

Search capabilities when included with hierarchy structure can make the collaborative application easier to navigate and access specific content. Searching for specific content may be a good design criterion to add when accessing hierarchies. Instead of utilizing a screen reader with Think Tank for text-to-speech capabilities, the DeskBot Genie is integrated with Think Tank to provide text-to-speech capabilities. The DeskBot Genie is a third-party application, which provides text-to-speech capabilities by 
reading any text content placed on the Windows' Clipboard Object. While navigating Think Tank's hierarchy, all text content is copied by the Think Tank application to the Windows' Clipboard Object and vocalized by the DeskBot Genie.

In order to evaluate Think Tank and the DeskBot Genie interfaces, a review of Think Tank was conducted. During the review, the hierarchy structure in Think Tank was difficult to follow when accessed with only the keyboard. The Think Tank hierarchy was navigated by pressing the tab, alt-tab, up-arrow and the down-arrow keys. Navigating from the categories to the ideas or from the ideas to the categories is accomplished by pressing the tab and alt-tab keys. Unfortunately, the tab sequence must follow the order of the controls on the web page. Tabbing from the ideas to the category can require the tabbing past command buttons and other controls before the focus for input reaches the category panel (Figure 14). Additionally, the DeskBot Genie's ("Bell Craft DeskBot," 2008) audio comments lag behind the tab press, so persons with disabilities would have to wait to determine focus of a control. The DeskBot Genie is seen in the bottom righthand corner of Figure 14.

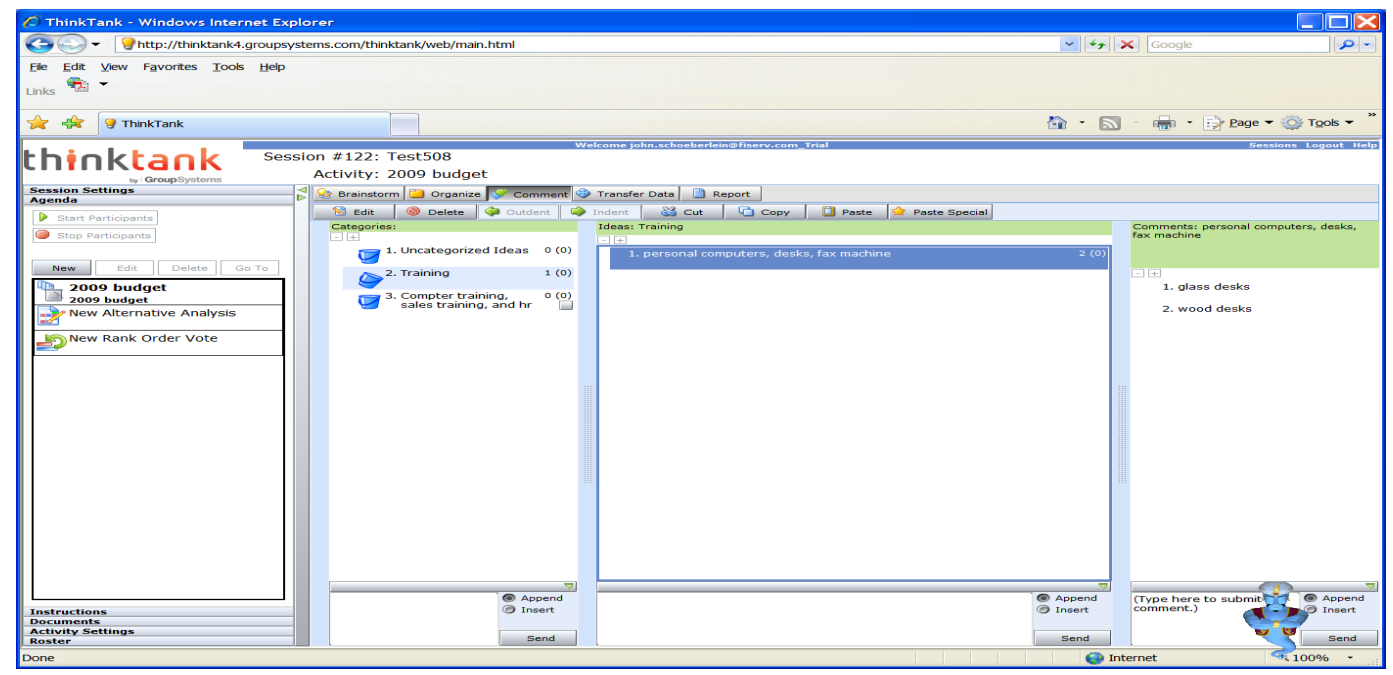

Figure 14 Think Tank Interface with the DeskBot Genie 


\subsubsection{Integrated Audio Input}

Integrated, built-in, audio input is available with Microsoft Outlook dictation and AOL AIM Voice Chat Room, but has to be setup by following an installation wizard and requires a microphone or a USB phone. The complexity of these setups may be difficult to follow and complete.

Integrated audio input is not available with Google Blog, Twitter, Google Docs, Microsoft SharePoint or Think Tank, but should be considered as an alternative input method for persons with disabilities.

\subsubsection{Integrated Text-to-Braille}

Integrated text-to-Braille is not available with any of the collaborative applications we studied. Text-to-Braille would provide a hard copy for the visually impaired to read at a later time, if necessary. Having a hard copy would be a valuable alternative to speech from a screen reader or a speech synthesizer. Therefore, text-toBraille should be considered to provide alternatives for displaying messages for persons with disabilities. Integrated text-to-Braille could be added via an API.

\subsubsection{Screen Reader}

The JAWS Screen Reader was utilized with Microsoft Outlook, Microsoft SharePoint, AIM, Google Blog, Google Docs, Twitter and Think Tank. Unfortunately, there were issues in two general areas: User Interface (UI) components; and, event handling (Mikovec et al., 2009). Often screen readers miss these events and the present state of components, such as a check box being checked (Mikovec et al., 2009). 
Accessible collaborative interface prototypes may be utilized in accessibility and usability studies to test out various solutions to inaccessible collaborative interfaces. The resulting design enhancement developed with the prototype can be delivered to the collaborative application developers and the screen reader developers for implementation.

\subsubsection{Keyboard Access}

The keyboard was utilized in conjunction with the JAWS Screen Reader. Since persons who are blind do not utilized the mouse for input, only the keyboard interface was utilized. Some level of keyboard access is supported by most of the collaborative applications studied in the form of short-cut keys and special keys (left-arrow, rightarrow, tab, and alt-tab) for navigation. The tab and alt-tab key presses can be used to transverse forward and backward. However, since the order of controls is pre-defined by the application, it may be difficult for persons who are blind to break the predefined order and go directly from point A to point B. Google Docs, Twitter and Think Tank present accessibility issues for persons with disabilities. For Google Docs, the keyboard cannot access the document-editing menu. For Think Tank, the keyboard key press is often outof-sync with the audio comments presented by the DeskBot Genie. For Twitter, the Twitter Menu is not accessible with the keyboard.

A summary of each collaborative application is provided in Table 9. Since these collaborative applications have different goals, they are not completely comparable. The goal for Table 9 is to present the features as they relate to each collaborative application and is not intended as a comparison between the collaborative applications. 
Table 9. Collaborative Applications Comparison (shared criteria comparison)

\begin{tabular}{|l|l|l|l|l|l|l|l|}
\hline Application & AIM & Outlook & SharePoint & $\begin{array}{l}\text { Google } \\
\text { Blog }\end{array}$ & $\begin{array}{l}\text { Google } \\
\text { Docs }\end{array}$ & Twitter & $\begin{array}{l}\text { Think } \\
\text { Tank }\end{array}$ \\
\hline Platform & Windows & Windows & Web & Web & Web & Web & Web \\
\hline API & API & API & API & API & API & API & None \\
\hline $\begin{array}{l}\text { Hierarchy / } \\
\text { List }\end{array}$ & Yes & Yes & Yes & Yes & $\begin{array}{l}\text { Yes, easy } \\
\text { to } \\
\text { navigate } \\
\text { using } \\
\text { search }\end{array}$ & $\begin{array}{l}\text { Yes, list } \\
\text { structure }\end{array}$ & Yes \\
\hline $\begin{array}{l}\text { Audio Input } \\
\text { Yes, }\end{array}$ & $\begin{array}{l}\text { Yes, } \\
\text { Chat }\end{array}$ & No & No & No & No & No \\
\hline $\begin{array}{l}\text { Rext-to- } \\
\text { Support }\end{array}$ & No & No & No & No & No & No & No \\
\hline $\begin{array}{l}\text { Screen } \\
\text { Adaptability }\end{array}$ & Yes & Yes & Yes & Yes & Yes & Yes & Yes \\
\hline Yeyboard & Yes & Yes & Yes & Yes & $\begin{array}{l}\text { Yes, } \\
\text { partial } \\
\text { access }\end{array}$ & $\begin{array}{l}\text { Yes, } \\
\text { access }\end{array}$ & Yes \\
\hline
\end{tabular}

\subsection{Discussion and Findings}

Although these applications provide access through the keyboard, many viable alternative methods are limited or not available. Text-to-Braille is not included with any of the applications reviewed. It is assumed that persons who are blind will use the keyboard in conjunction with a screen reader, like JAWS ("Freedom Scientific JAWS," 2008); to interact with the application's interfaced. 
Popup message boxes can be disorienting for persons with disabilities. Auditory signals or messages should be included with popup message boxes, to alert persons with disabilities of the popup.

Hierarchy organization of information is the structure most often used in these applications, and has been found in all the applications reviewed. However, hierarchies of multiple tiers (deep structures) beyond three levels will be difficult for persons with disabilities to navigate and there is a good chance of the users becoming lost or disoriented (Kiger, 1984). The benefits of hierarchies are: they help to categorize items into common lists; and, they can be utilized to speed up search time. An example of a hierarchy would be to categorize cars based on manufacturer, such as Honda, BMW, Toyota, and Ford. An advantage to hierarchies is that a hierarchy enables direct access to specific content. For example, selecting the "Ford" hierarchy item could directly link to all Ford Automobiles. The drawback to hierarchies is in building a hierarchy that is too deep with too many levels. The deeper the level, the more confusing the location may seem to the person searching the hierarchy. The key to well-developed hierarchies is to keep the depth at about three levels, to avoid confusion (Kiger, 1984). 
3.8 Potential Approaches for Developing Accessible Prototypes

How can the accessibility and usability issues presented in this chapter be resolved? The following sub-sections offer three approaches for implementing accessible and usable collaborative applications.

\subsubsection{Application Programming Interface (API) for Prototype Development}

Most of the applications examined, including Microsoft Outlook ("Microsoft Outlook Object Library,” 2008), AIM (“AIM Object Library,” 2008), Twitter (“Twitter API,” 2009), Microsoft SharePoint (“Microsoft SharePoint Services 3.0,” 2009) and Google Blog (“Google Blogger Data API,” 2008), supply an Application Programming Interface (API), which can be invoked by accessible collaborative interface prototypes for usability testing purposes. Accessible collaborative interface prototypes could include support for event handling, navigation, text-to-Braille, and flattened hierarchies, to improve accessibility and usability. The intention of developing a prototype utilizing the API is to expand upon the capabilities of the original interface and to provide accessible and usable interfaces. Table 10 provides some suggested advantages and disadvantages of the API approach. 
Table 10. Advantages and Disadvantages of the API Approach

\begin{tabular}{|l|l|l|}
\hline Technique & \multicolumn{1}{|c|}{ Advantages } & \multicolumn{1}{c|}{ Disadvantages } \\
\hline API & $\begin{array}{l}\text { 1. } \begin{array}{l}\text { Single application layer } \\
\text { supporting navigation and } \\
\text { events }\end{array} \\
\text { 2. Easy to develop }\end{array}$ & $\begin{array}{l}\text { 1. Requires API } \\
\text { 2. Developed on a per } \\
\text { application basis }\end{array}$ \\
& $\begin{array}{l}\text { 3. Supports Web, console and } \\
\text { Windows applications } \\
\text { 4. Application-level solution } \\
\text { purposes. }\end{array}$ & \\
& $\begin{array}{l}\text { 5esign can be delivered to } \\
\text { JAWS and collaborative } \\
\text { Applications' Developers, } \\
\text { to implement }\end{array}$ & \\
\hline
\end{tabular}

\subsubsection{Common Middleware Layer Approach}

A common middleware layer is added in-between the screen reader/browser layer and the Web-application layer, using WAI-ARIA metadata (Figure 15). The screen reader application and the browser have to be compatible with WAI-ARIA metadata. JAWS Version 10, using Microsoft Internet Explorer version 8 or Mozilla Fire Fox version 3 Browsers and more recent versions, recognize WAI-ARIA metadata ("The Paciella Group Blog,” 2009). The WAI-ARIA metadata describes components (i.e. Command buttons, trees, check boxes, etc.) and events (i.e. notify or ignore), to provide a connection between collaborative applications and the screen reader/browser. 
Screen Reader (JAWS) and Web Browser (Internet
Explorer or Mozilla Fire Fox)

WAI-ARIA metadata

Web Application

Figure 15 Screen Reader Middleware Layer using WAI-ARIA

Table 11 provides some suggested advantages and disadvantages of the

Middleware Layer approach.

Table 11 Advantages and Disadvantages of the Middleware Layer Approach

\begin{tabular}{|c|c|c|}
\hline Technique & Advantages & Disadvantages \\
\hline $\begin{array}{l}\text { Middleware Layer (WAI- } \\
\text { ARIA) }\end{array}$ & $\begin{array}{l}\text { 1. Recognized by W3C } \\
\text { as a standard } \\
\text { 2. Operating system- } \\
\text { level solution } \\
\text { 3. May be applied } \\
\text { universally }\end{array}$ & $\begin{array}{l}\text { 1. Web-based } \\
\text { solution only } \\
\text { 2. Difficult to } \\
\text { change, since there } \\
\text { are three-levels } \\
\text { involved in } \\
\text { changes } \\
\text { 3. All three layers } \\
\text { have to work in a } \\
\text { coordinated effort } \\
\text { to provide event } \\
\text { handling and } \\
\text { navigation support } \\
\text { Not accessible yet } \\
\text { (The Paciella } \\
\text { Group Blog, 2009) } \\
\text { May be difficult to } \\
\text { understand the } \\
\text { logic by } \\
\text { developers, since } \\
\text { there are three } \\
\text { levels interacting } \\
\text { to provide a } \\
\text { solution }\end{array}$ \\
\hline
\end{tabular}




\subsubsection{Screen Reader Modifications Approach}

Another approach is to utilize the Non-visual Desktop Access (NVDA) ("NonVisual Desktop Access," 2009) Screen Reader for the Windows Operating System. The NVDA Screen Reader is an open source free-of-charge screen reader that provides speech and Braille output. This screen reader runs entirely from the USB Drive, so there is no need to install the NVDA Screen Reader onto the computer's hard drive. Additionally, since NVDA Screen Reader is open source, the screen reader could be developed to interface a collaborative application's object library to provide collaboration features.

\subsection{Conclusion}

The "Collaborative Technology Accessibility Evaluation Framework," was utilized to map out an approach for evaluating collaborative applications' accessibility and usability. Once the collaborative applications were selected for evaluation, each application was evaluated using either Web-based evaluation criteria or Windows-based evaluation criteria or both. The results of these evaluations were that each application evaluated was not fully accessible. Navigation and random events (i.e. popup boxes and dialog boxes) were issues that prevent access and use by persons with disabilities.

The accessibility issues of collaborative applications prevent persons who are blind and other persons with disabilities access to these highly graphical interfaces. Although these applications met the accessibility requirement by enabling keyboard access and screen reader access, most applications provide only minimum access. From this evaluation, it appears that these applications are not usable, even though basic accessibility requirements were considered in the application design. 
Three approaches including accessible collaborative interface prototypes, middlelayer approach such as WAI-ARIA, and a modified version of NVDA Screen Reader were proposed suggestions for providing accessibility and usability to collaborative applications. An accessible collaborative interface prototype was the approach utilized for the development of a solution for this dissertation.

Accessibility can be improved by including a list of choices, flattened hierarchies, text-to-Braille, event handling, navigation, and speech recognition. Much work remains to make collaborative applications accessible and usable. Additionally, involvement from persons with disabilities is needed in future studies. 


\section{INITIAL FIELD VISITS TO THE NATIONAL FEDERATION OF THE BLIND}

\subsection{Introduction}

Several visits were conducted at the National Federation of the Blind (NFB) ("National Federation of the Blind," 2008) in order to tour the technology lab and to identify the logistics for a proposed pilot focus group study.

During one visit, a member from the National Federation of the Blind provided a tour, an interview session and an opportunity to observe them utilizing Microsoft Outlook 2003 with the JAWS screen reader. For the purposes of this discussion, we will identify this person as Participant-1. The findings based on the discussion and observation of Participant-1 interacting with Microsoft Outlook is summarized in the following sections. At the conclusion of this visit, a plan was developed to recruit participants with the assistance of the NFB for a pilot focus group study.

\subsection{Applications that Support Group Work}

When discussing the applications utilized by the teams at NFB to support group work, MS Outlook and MS Word were identified as the most frequently used applications. In addition to emailing messages, Microsoft Outlook is utilized for scheduling meetings. Microsoft Word is utilized for document co-editing and sharing. At the time of our visit, applications such as AIM, WIKI and blog were mostly utilized for personal use. To fulfill the needs of documenting and idea sharing, plans are underway to utilize WIKI technology. However, none of the traditional full-fledged groupware was mentioned during discussion. 


\subsection{User Population at the NFB}

The typical end-users of these applications at the NFB are mainly young employees who are pushing for new technology. Some older employees may not be as advanced with utilizing technology. All members of the Access Technology are capable users of screen readers and applications utilized to support group work (e.g. Microsoft Outlook, Microsoft Word, etc.).

\subsection{Adaptive Technology}

GW Micro’s Braille Sense (2008) and Freedom Scientifics Pac Mate (2008) are the Braille keyboard technologies used by Participant-1 for typing notes and reading output. Touching the protruding pins of the Braille keyboard, Participant-1 is able to read the output of the device. In addition to the Braille keyboard, Participant-1 utilizes the JAWS screen reader to receive audio output. Both of these adaptive technologies are necessary for Participant-1 to interact with computer applications.

\subsection{How a Typical Project is handled in Groups}

The typical work group at the NFB includes members who are blind and sighted. Although most groups are co-located, there are instances of virtual groups as well. For both groups, synchronous and asynchronous communications are utilized. The project process is the same whether the participants are blind or sighted, just the technologies (i.e. screen readers, note takers, etc.) utilized may vary.

A project leader generally manages all group work conducted at the NFB. The project manager initiates the project by inviting group members to a face-to-face kickoff meeting. This kickoff meeting is conducted in a conference room, with one member of the group taking notes. After the meeting, additional meetings are scheduled and notes 
and documentation are exchanged through email messaging. Documentation is co-edited with comments and track changes, using Microsoft Word.

The following accessibility and usability issues related to Microsoft Word's track changes' and comments' features were discussed:

1. Searching the Microsoft Word Ribbon Menu for track changes are time consuming and are difficult to find;

2. Comments are difficult to differentiate from the text of a sentence or paragraph;

3. Multiple track changes in the same sentence or paragraph are difficult to differentiate; and,

4. Accepting track changes are difficult without the context of the track change.

Generally, the NFB Accessibility Team Members schedule meetings and communicate all project requirements via email using Microsoft Outlook, and document their plans using Microsoft Word.

\subsection{Microsoft Outlook Observations}

In order to gain an understanding of the accessibility and usability issues encountered, the researcher observed Participant-1 interacting with Microsoft Outlook and as time allowed questions were asked to clarify the events observed. Questions such as "How do you access the calendar for scheduling, the inbox or other folders to review email messages and navigate the hierarchy of folders in the Microsoft Outlook interface?" were discussed.

During the observations of Participant-1 using Microsoft Outlook, the following items were observed: the inbox was the focal point of the interface, with the navigation and reading panes removed from the interface; and, the JAWS Screen Reader and the 
keyboard were the devices used for accessing the emails. Participant-1 mentioned that the original interface of Microsoft Outlook (Figure 16) was too cluttered to properly navigate, because the screen reader always reads all of the text on the interface from upper left to bottom right without regard to the logical connection between the texts.

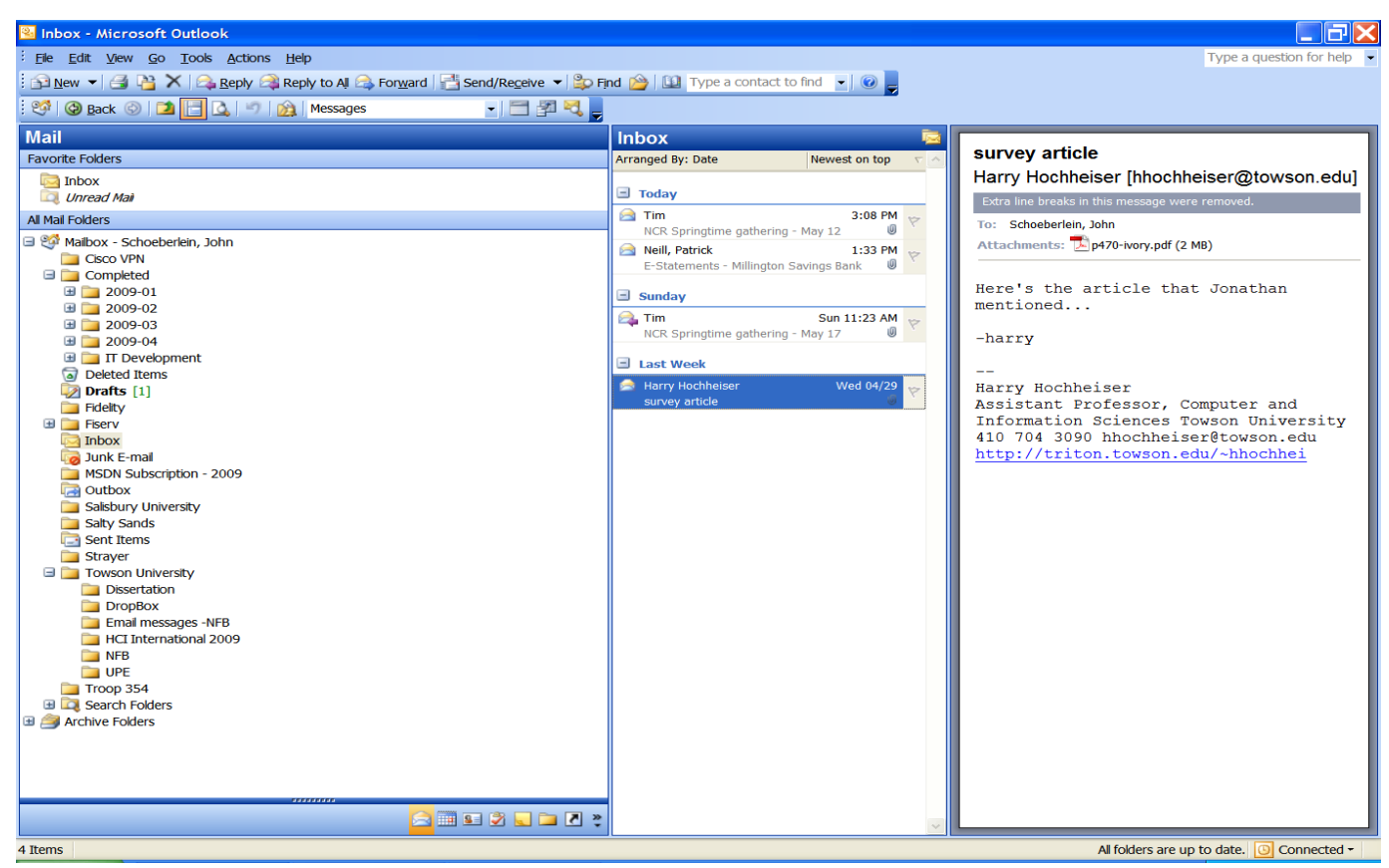

Figure 16 Microsoft Outlook Interface

Consistent with the discussion on the principle of designing accessible interfaces (Luk, et al., 2000), Participant-1 preferred simple interfaces that only presented the most important information. Therefore, when there was too much functionality/information provided on one interface (i.e. Microsoft Outlook), Participant-1 tried to find a way to simplify the interface so that it was easier to navigate. Participant- 1 was able to achieve this by removing complex folder structures and panels (i.e. the reading pane and navigation pane) found with Microsoft Outlook (Figure 17), so the content of the interface pertained to a specific function (i.e. email messages, or contacts, etc.). 


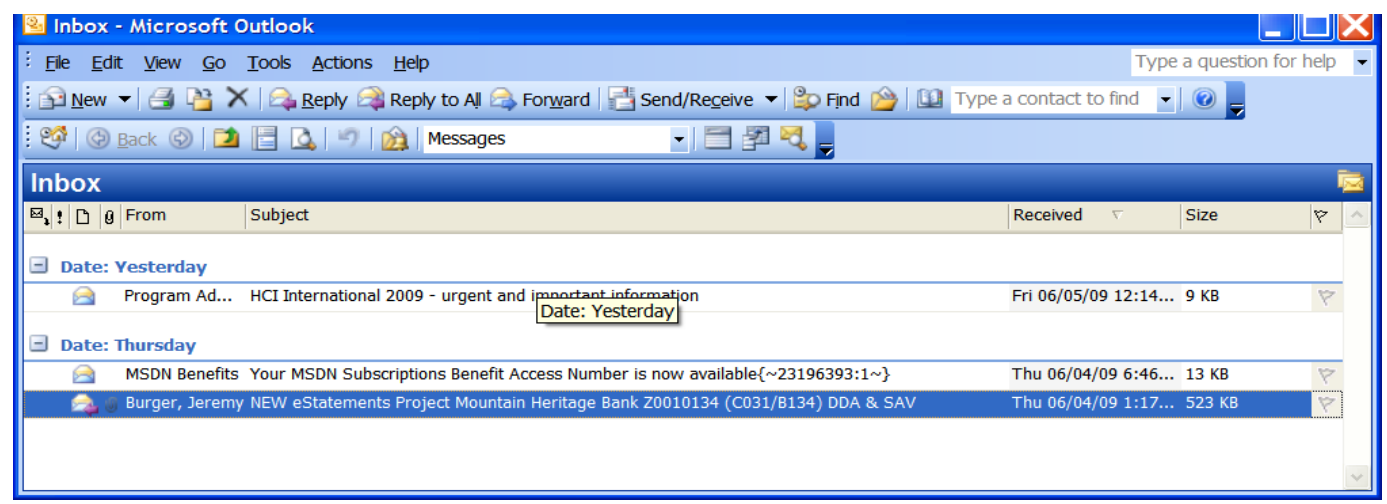

Figure 17 Microsoft Outlook with navigation and reading panes removed

Participant-1 was observed using the JAWS Screen Reader to sequentially read each email message's subject line without the clutter of the folders and details of the messages. To navigate the folder hierarchy and to select a different folder (i.e. Output, Inbox, etc.), the control-y keys are pressed simultaneously. Hiding and showing the hierarchy of folders enabled greater accessibility and usability, since the interface was less cluttered and the screen reader interacted with less content.

Accessibility and usability were improved by reducing the complexity of the interface by removing complex hierarchies; simplifying the interface by separating each function (i.e. read email messages, schedule appointments, assign tasks, etc.) on a separate interface; providing keyboard access; and, providing some form of text-tospeech output via a screen reader.

\subsection{Conclusion}

The visits to the NFB shed some light on how persons who are blind perform group work and the support they require. However, these visits were just preliminary. Therefore, additional field studies and focus group studies were conducted in order to fully understand how persons who are blind use collaborative applications, what kind of 
accessibility and usability issues they experience with these applications, and, to explore a solution to help solve the accessibility and usability issues identified. 


\section{PILOT FOCUS GROUP STUDY}

\subsection{Introduction}

In an initial effort to understand how persons who are blind work in groups, the collaborative applications utilized and accessibility and usability issues encountered, a pilot focus group study was conducted to answer these questions with the assistance from the National Federation of the Blind (NFB), in Baltimore, Maryland, in the winter of 2009.

The following research questions were addressed during this study:

- RQ1: How do persons who are blind work in groups including different scenarios?

- RQ2: What is the accessibility and usability challenges persons who are blind experience with software applications (specifically collaborative systems)?

- RQ3: What kind of support do persons who are blind require so that they can perform group work?

\subsection{Research Method and Procedure}

Focus groups are group interviews (Courage \& Baxter, 2005). Generally, a focus group is utilized for marketing research purposes while it has been used in social and design research since the 1930's (Courage \& Baxter, 2005). Focus groups are very useful for the generation of ideas and for discovering problems, challenges, frustrations, likes, and dislikes among participants especially when the researchers do not have enough information to design a survey. The results from a focus group study can provide information to prepare for other studies such as field studies and usability studies. Moreover, focus groups allow for the access of multiple points of view in a short time 
period (e.g. a single meeting) (Courage \& Baxter, 2005). The size of the group can vary from 4 to 6 as a mini-group to more than 10 as a full group (Greenbaum, 1998).

Considering the explorative nature of this particular study, a focus group is an appropriate approach to gather data regarding collaborative applications from persons who are blind and work in groups. Focus groups allow data collection from multiple sources at a single meeting, observation of the group dynamic as they interact, gained agreement among several group members, and validation of the group process and other data with the entire group.

The focus group study session, contained the following steps:

1. Recruit the participants;

2. Prepare demographic, prior-experience questionnaire and meeting guideline;

3. Conduct the focus group sessions;

4. Perform content analysis of the audio transcript and the researcher notes, to identify content categories;

5. Validate agreement of the coded content, using Cohen's Kappa (Cohen's Kappa, 2010); and,

6. Report findings.

Prior to the pilot focus group study, a total of five participants filled out a short questionnaire, regarding demographic information and prior-experiences with collaborative applications and screen readers. A meeting guideline was designed, before the start of the meeting, containing a list of possible questions for discussion.

In order to be easily accessible for the participants, the focus group session was conducted in a private conference room at the NFB. The author served the moderator and 
note taker, while an assistant served as a duplicate note-taker during the session.

Additionally, the focus group session was audio recorded. The focus group started with a brief introduction from the moderator on the purpose of the meeting, a round of brief selfintroductions, and followed by a discussion on several topics prepared before the meeting. Follow-up, probing questions were asked to ensure the understanding of the discussion. The focus group session's duration was one hour and thirty minutes.

Following the focus group study, the notes and the audio recording of the group discussion were transcribed with the permission from the participants. The audio transcript and detailed notes from the group discussion were coded based on the themes that emerge from the participants' comments.

\subsubsection{Participants}

As Geobert and Rosenthal (2002) noted, the "primary consideration is who will provide the most insightful information" related to the topic when conducting a focus group or field study. Considering the objective of this research project, the criteria for selecting participants are defined as follows:

- Persons who are blind, with no residual vision;

- Persons who work in group settings;

- Persons who are familiar with screen readers;

- Persons who utilize computers for their work; and,

- Persons who have tried to use collaborative applications and other tools to support his/her collaboration with others. 
The Researcher solicited help from the NFB, in order to recruit persons who are blind to participate.

\subsection{Data Analysis Method - The Grounded Theory Criteria for Evaluation}

The Grounded Theory (2009) was utilized to help interpret the audio data recorded during the study sessions. The Grounded Theory method follows this general path: code key points discussed, group the key points into similar themes and categories, and create theories from the categories to explain the data (Grounded Theory, 2009). In order to code key points and derive categories, thematic analysis of free-flowing text data method (Grounded Theory, 2009) was applied to the transcript data from the focus group studies and field studies. The main steps of the thematic analysis of free-flowing text data are as follows: development of a cookbook; establish Inter-coder reliability (Kurasaki, 2000); and, apply the cookbook systematically to the data (Grounded Theory, 2009).

\subsubsection{Development of a cookbook}

Development of the cookbook consists of the following steps: annotate the text of the transcript; sort the annotated list; label the thematic categories; and, assign a numeric code to the categories (Grounded Theory, 2009).

The first step was to develop an annotated list of the margin notes taken while analyzing the transcript from the Focus Group Studies and field studies. The annotated list was sorted into common themes or categories. Finally, the Categories were identified from the grouping of the annotated list elements. 


\subsubsection{Establishing Inter-coder Reliability}

Establishing inter-coder reliability consists of the following steps: train and calibrate coders; code data; calculate inter-coder agreement (Kurasaki, 2000). Two research assistants were asked to work as coders. Prior to coding the transcripts, each coder was trained in order to understand the meaning of the codes and the method of applying the codes to the transcript. The sample contained various discussions randomly extracted from several pages of the transcript, and served as the training materials for the coders.

During the training, the coders were instructed to highlight the information related to the categories. A category number was added above each individual piece of text highlighted in the transcript from the focus group study. For example, if the coder highlighted the text "Outlook", they would mark a "1" above the highlighted text in the transcript. If the coder was not sure which category the highlighted text belonged to, they would write down the category number they thought the highlighted text matched the closest, and then they would write down all the category numbers they thought the highlighted text may belong to.

After explaining how to do the coding, each coder coded the training material individually. The codes were then compared. When a disagreement was identified between the two sets of codes, the coders meet and discussed how they determine their code until a consensus was reached. This process continued until the coders felt confident that they understood the meaning of each code and how to properly identify lines that represented the codes in the transcript sample. After the training exercise was complete, to the satisfaction of the two coders, the actual transcript was coded. 


\subsubsection{Calculate Inter-coder Agreement}

Agreement among Coders for each category was calculated by using a ratio of agreements versus disagreements. The higher the ratio, the more similar Coders identified the text as being in the same category and a greater reliability between Coders. All lines of the coded text were selected from the transcripts for statistical analysis. Statistical analysis on the agreement between the Coders was calculated using SSPS ("SSPS Inc.," 2010), using Cohen’s Kappa (2010). 
5.4 Results from the Pilot Focus Group Study

\subsubsection{Demographics}

The participants of the focus group study included five members of the NFB. The Access Technology and Education Team was an actual group of the NFB, responsible for evaluating applications for accessibility and usability and providing technical and education support in the use of the applications utilized by the NFB.

The five participants consisted of three females and two males. All members are between 20 and 39 years old. All of the participants utilized the computer daily, for business use, and have utilized the computer between 11 and 20 years. All participants utilize the JAWS Screen Reader ("Freedom Scientific JAWS," 2008) with two participants having 6 to 10 years' experience and two participants having 11 to 20 years' experience. The fifth participant did not respond to the number of years' experience with the JAWS Screen Reader. None of the participants reported that they ever used any traditional full-fledged groupware applications, so the researcher focused on collaborative applications that include some groupware features such as: shared calendar, email, track changes, and Chat. The participants were asked to identify all the collaborative applications they have used for group work. As the result, Microsoft Outlook (application version 2008) (“Microsoft Outlook," 2008) was identified as the primary collaborative application utilized most frequently in completing group projects at the NFB. One participant had 1 to 5 years' experience; three participants had 6 to 10 years' experience and one participant had 11 to 20 years' experience using Microsoft Outlook. All 
participants used Microsoft Outlook daily. When interacting with Microsoft Outlook's interface, the participants utilized the JAWS Screen Reader exclusively.

Collectively, all participants of the focus group study had experience with the Windows Operating System, the JAWS Screen Reader, and interacting with Microsoft Outlook when conducting group projects.

\subsubsection{Content Analysis of the Focus Group Study Recording}

Content analysis was performed on the audio transcript and researchers' notes from the group discussion collected from the focus group study, identifying seven distinct categories as a result. The common themes or categories with their associated annotated list elements are shown in Table 13. 
Table 13. Categories and Annotated List Elements

\begin{tabular}{|c|c|}
\hline Category & Annotated List Element \\
\hline $\begin{array}{l}\text { 1. Participant's identification of } \\
\text { collaborative software utilized }\end{array}$ & $\begin{array}{l}\text { a. Features of collaborative applications } \\
\text { utilized; and, } \\
\text { b. Collaborative applications utilized } \\
\text { when conducting group projects. }\end{array}$ \\
\hline $\begin{array}{l}\text { 2. Tasks/steps necessary to complete group } \\
\text { projects }\end{array}$ & $\begin{array}{l}\text { a. Frequency of group meetings; } \\
\text { b. Group message passing techniques; } \\
\text { c. Tasks necessary to complete a group } \\
\text { project; } \\
\text { d. Frequency of the tasks of a group } \\
\text { project; and, } \\
\text { e. Determining project priorities. }\end{array}$ \\
\hline 3. Accessibility and usability issues & $\begin{array}{l}\text { a. Accessibility issues encountered } \\
\text { when using collaborative applications; } \\
\text { b. Issues encountered when trying to } \\
\text { utilize new applications; } \\
\text { c. Issues encountered when using the } \\
\text { JAWS Screen Reader; and, } \\
\text { d. Most severe accessibility issues } \\
\text { reported; }\end{array}$ \\
\hline $\begin{array}{l}\text { 4. Collaborative applications utilized but } \\
\text { discontinued }\end{array}$ & $\begin{array}{l}\text { a. Applications that group members } \\
\text { stopped using; } \\
\text { b. Collaborative applications not } \\
\text { pursued; and, } \\
\text { c. Reasons for not attempting new } \\
\text { applications. }\end{array}$ \\
\hline $\begin{array}{l}\text { 5. Applications/Tools utilized to support } \\
\text { group work }\end{array}$ & Other applications utilized. \\
\hline 6. Accessibility design considerations & $\begin{array}{l}\text { a. Proposed solutions for issues } \\
\text { encountered; and, } \\
\text { b. Suggested improvements for screen } \\
\text { access software. }\end{array}$ \\
\hline 7. Accessibility documentation and support & $\begin{array}{l}\text { a. Collaboration support needed; and, } \\
\text { b. Support needed for accessibility } \\
\text { features of applications. }\end{array}$ \\
\hline
\end{tabular}


The Cohen's Kappa measurement of agreement sought was $70 \%$ or greater, to provide evidence of acceptable agreement and reliability between the Coders ("Cohen's Kappa," 2010). Among 86 cases identified, the Coders agreed on $75.7 \%$ of those cases providing evidence of acceptable agreement and reliability between the Coders. The following sections will present each of the seven categories identified with discussions.

\subsubsection{Participants Identification of Collaborative Software Utilized}

During the focus group study at the NFB, it was discovered that the participants did not utilize any traditionally identified groupware (e.g. Lotus Notes); therefore, our discussion was focused on collaborative applications that may contain some groupware features. Unsurprisingly, similar to the answer obtained from the short background questionnaire, the primary collaborative application reported by the focus group participants was Microsoft Outlook for various activities including: email, journal, calendar, contacts, and tasks. While the participants reported using the calendar to schedule for group meetings and using the journal feature to track certain projects, most of the group work is being done by exchanging emails within the group. At this point, Microsoft Outlook was identified as having a major role in supporting group collaboration at the NFB. 


\subsubsection{The Tasks/Steps Necessary to Complete a Group Project}

In addition to understanding the collaborative applications utilized, it was important to identify the tasks or steps utilized when completing a group project. Understanding the group process steps helps to identify the tasks that need collaborative support. The participants reported that they start a group project with a face-to-face "brainstorming session" in a conference room. Conferencing calls are normally made to get the project started, in the situation when group members involved in the project are in another location. During the face-to-face meeting or conference call, a member of the group is normally responsible for note taking. Once the start-off meeting ends, the group's manager sends emails with documents containing the ideas discussed. A project schedule and task list is developed by the manager and sent through Microsoft Outlook, as either an email or calendar items. The group then exchanges emails to discuss further on the project. Weekly or monthly face-to-face meetings or conference calls are conducted throughout the project duration. Based on this group process, support is needed for call conferencing, exchanging emails, note taking, and document editing and exchange.

\subsubsection{Accessibility and Usability Issues Experienced}

Since the focus group participants identified Microsoft Outlook as a major application utilized to support group work, Microsoft Outlook's accessibility and usability issues are presented. Based on this initial research, it appears that Microsoft Outlook used in conjunction with the JAWS Screen Reader has both accessibility issues 
and usability issues. The next paragraphs discuss the accessibility issues and usability issues discovered during the focus group study.

\subsection{Accessibility Issues}

The participants reported accessibility issues with several of the features provided by Microsoft Outlook. Most of the participants agreed that the web-based version of Microsoft Outlook is inaccessible and instead the group utilizes the application version of Microsoft Outlook. Therefore, all the comments are focused on the Windows-based application version of Microsoft Outlook.

The accessibility issues identified for Microsoft Outlook include: inconsistency issues between Microsoft Outlook and the JAWS Screen Reader; reading the wrong message by the JAWS Screen Reader; and, accessibility issues with the Journal, calendar, messages, login screen, and contacts. The participants reported that the most significant accessibility issues are in regard to the monthly-view of the calendar control within Microsoft Outlook's Appointment task.

The first challenge the participants face is the inconsistency of the accessibility of the various features of Microsoft Outlook, as well as the interactions between the screen reader and the application. For example, the participants commented, "Some days it (screen reader) works, some days it does not work. Whether it works, depends on the individual machine." After further discussion, it was realized that the problem may be attributed to the version of the operating system, the version of Microsoft Outlook, or the version of the JAWS screen reader installed on each individual machine. If each machine is running the same version of the operating system, Microsoft Outlook, and the JAWS 
Screen Reader, the inconsistency of the accessibility may be resolved. More research is needed to identify the root cause of the problem.

Another accessibility issue identified by the group is related to the software upgrade and version of the screen reader. For example, participants commented on the problem they experience when trying to switch views in "calendar" feature, "It worked with JAWS 10. It is not working with JAWS 11". It appears that upgrading the JAWS Screen Reader to a newer version may cause inconsistency issues with collaborative applications. These inconsistencies caused by upgrades to the JAWS Screen Reader may make it harder for persons who are blind to adapt to the changes in the interface. In this case, utilizing the daily-view of the calendar was a work-around for the inaccessibility of the monthly-view of the calendar. In some inaccessibility cases, often the solution may be to approach the interface from another direction, such as, utilizing a different view (dailyview versus month-view) to access information.

Since the first step to utilizing Microsoft Outlook is to log into the system, it is necessary that the login screen be accessible. If the login screen is not accessible, the whole system will not be accessible. A participant commented, “Occasionally, the Login Screen (Microsoft Outlook) will not read correctly (using the JAWS Screen Reader). I have to set Outlook to work offline and then login again." The participants proposed a work-around solution to the accessibility issue of the Login Screen, which is to uncheck the work-offline check box within Microsoft Outlook's interface. A permanent solution for the inaccessibility issues the Login Screen is needed. 
The participants ranked the "calendar" feature of Microsoft Outlook as being the most inaccessible feature of Microsoft Outlook. The participants expressed that they did not receive notifications of important events, such as meetings or appointments. As a result of not receiving any notification of a meeting or appointment, they may miss an important deadline. The JAWS Screen Reader may need to be enhanced to respond to events, such as the notifications of meetings or appointments. More research is needed, to discover possible approaches to event notifications between any application and the screen reader.

The participants also reported that the multiple panels utilized in Microsoft Outlook presents a big challenge for screen readers. The participants had to move from one panel to another panel, to complete certain tasks. One method to work-around the movement between multiple panels was to display on the interface one panel and to hot key directly to other panels. The adjustment of the interface to present one panel of the interface simplified the complexity of the interface and reduced the amount of information presented to the screen reader. The ability to view certain information and being able to hide that information and view other information is an important feature for accessible interfaces.

\subsection{Usability Issues}

The Participants identified usability problems of the applications they use during the discussion. Even though there is distinction between accessibility and usability, some of the usability problems can make the system inaccessible. For example, when discussing the problems encountered when they try to use the "Journal" feature of 
Microsoft Outlook, they commented, "(the problem is) in searching the time line.

Sometimes it is not an accessible experience."

The participants ranked the "calendar" feature of Microsoft Outlook as being the most unusable feature of Microsoft Outlook. The participants complained that they were not able to, "share the calendar." Additionally, they commented that appointments were not tracked correctly. The calendar is a group feature that must function properly, or meetings, appointments and project target dates may be missed.

When discussing the "email" feature of Microsoft Outlook, the participants noted that they may not receive a message, or the message may not read correctly. A Participant commented that the messages are not up to date, "sometimes I have to refresh. The new message is on the screen, but it is still reading the old message." The screen reader must properly and consistently read messages in the order they are delivered in order for any group, dependent on a screen reader, to respond and function as a team. Observing the participants' interaction with email messages may lead to the root cause of the issues while reading messages.

The "contact" feature of Microsoft Outlook presents another challenge for the group participants. They reported that, "it keeps reading the same contact (even though I know I have kept more contacts in the system).” The minimum requirement of the screen reader should be to identify an entire list and to be able to parse that entire list in any order. This feature may not function due to the presentation of the contacts on the interface that the screen reader is reading.

Additionally, the reading pane had to be removed from view in order for the JAWS Screen Reader to work in a manner that the participants could understand. 
Basically, the interface worked better with the JAWS Screen Reader, when the interface was less cluttered.

\subsection{Summary of the Issues}

There are a number of accessibility issues reported by the Access Technology and Education Team, but for the most part they appear to be related to how the screen access software interacts with the graphical interface. Some of the accessibility and usability issues seem to pertain to synchronization issues between the screen reader and the content. The accessibility and usability issues included: inconsistency issues between Microsoft Outlook and the JAWS Screen Reader; reading the wrong message by the JAWS Screen Reader; missing notifications of meetings; inability to login; sharing the calendar; and reading the same contact, to name a few examples. These examples present opportunities that can be corrected to provide and accessible and usable experience for persons who are blind.

\subsubsection{Collaborative Applications Utilized, but Discontinued}

The focus group identified collaborative applications attempted for group work and discontinued. The goal was to identify the reasons why applications were discontinued and, if there was a common set of reasons.

The Access Technology and Education Team have tried a number of collaborative applications, such as: WIKI, Survey Monkey, and Microsoft Access. However, they have identified several reasons for their discontinued use of these applications. The reasons include previous unsuccessful experience, lack of interest and additional learning curve. It appears that the focus group participants are apprehensive to use technologies that they 
have not tried, due to their previous unsuccessful experience with other software. For example, a participant commented that they are, "Easily intimidated by the other tools or they are not accessible. When you branch out, you may not get the best accessible tools." Unlike the widely used discussion forums, such as: group project discussion forums or class discussion forums, utilized among persons without visual impairment, the participants expressed a lack of interest in such applications, "it does not lend itself to the work environment." The extra learning curve was another reason noted for not attempting new applications, "When you learn to use the computer, you have to learn three things: the operating system, the application and the screen reader software. Sometimes it is just not worth it (to learn new software). Then, there is the extra learning curve." Anytime persons who are blind attempt to utilize a new collaborative application, they have to orient the screen reader to the interface of the application and learn the nuances of the application.

\subsubsection{Other Tools Utilized to Support Group Work}

In addition to Microsoft Outlook, the following tools are utilized occasionally by the Access Technology and Education Team: America Online (AOL) Instant Messaging (IM), for quick messaging; Microsoft Word, for document sharing; WIKI, for document storage and sharing, Twitter and Facebook, for social networking; and, Mobile Phones for, text messaging. As a Team, the Access Technology and Education Team utilize a small number of applications, when working in their group. The key to the limited use of other applications may be due to the accessibility and usability issues experienced, and the difficulty of learning new applications. 


\subsubsection{Accessibility Design Considerations}

During the focus group study session, the group offered suggestions on the design considerations or, support needed to make collaborative applications accessible. The participants emphasized the need that the screen access software needs to be consistent when interacting with the application's interface. On the other hand, the interface layout should be designed with screen readers in mind. Considering some of software manufactures developed interfaces that provided voice-over to their application to provide accessibility, a question regarding the participants' preference on voice-over interfaces versus using a screen reader was asked. The voice-over interface application choice was determined to be an unacceptable solution by the focus group participants. The participants commented, "Understanding how to use the self-voicing application in conjunction with the screen access software for other applications would be a hassle." Even though the screen reader is not perfect, the use of one screen reader for all applications was determined to be easier and the preferred approach for accessibility and usability.

\subsubsection{Suggestions on Accessibility Documentation and Support}

The documentation and support concerns identified and mentioned by the group include the low priority accessibility seems to receive by corporations, their developers, and their support staff, the accessibility documentation is often limited to only a few pages, and the support staff often does not understand the accessibility features of an application they are supporting or the support staff does not know the accessibility features even exist. 
The participants agree that even though most software vendors are aware of the Section 508 of the Rehabilitation Act, support for accessibility features is extremely limited compared to the support provided for other features. A participant noted, "Normally, to learn about accessibility features, you have to dig down pretty far to know whether an application supports accessibility." In most cases, there are few documents on accessibility features that, "They (accessibility features) are generally a small statement in the back of the documentation." These problems may be caused by the reluctance of the design team, "They really don't think about the user group they are serving. They do accessibility as a matter of course - as a requirement." Therefore, "there is a responsibility of the developers that they provide the same support for accessibility features."

The participants also suggested that the support staff of collaborative applications need to be trained on accessibility issues as well, "If I go home today and call Apple and say I need help with voiceover, 9-times-out-of-10, they (support staff) will not know what I am talking about. Are accessibility features not important enough to train their support staff on?" 


\subsection{Discussion}

Since the focus group participants are blind, this research provides a unique perspective on the group process conducted entirely with blind participants. It is important to note that the focus group participants are considered advanced users, and additional input from various experience levels is required.

The first research question "How do persons who are blind work in groups?" was answered during the pilot focus group study. The participants agreed that the normal group projects follow this general pattern: face-to-face/teleconference group discussion in a conference room setting; and, follow-up discussions and document exchanges utilizing email (e.g. MS Outlook). The most frequently utilized features include: the calendar for appointment tracking; email for information exchange, the journal for notes; the contacts for contact information; and, the tasks for tracking the progress of a project. Although the group process may appear similar to be a standard group process with meetings and exchanges of email, the difference is that persons who are blind require assistive technologies (e.g. screen readers and note takers) for exchanging ideas.

The second research question "What are the accessibility and usability challenges persons who are blind experience with software applications, specifically collaborative applications?" was answered during the pilot focus group study. As more and more applications have made their way to become web services, researchers have been focusing on the accessibility of web interfaces. For instance, Harrison (2005) had identified approaches to making web pages accessible but did not report approaches to improving the accessibility of windows-based applications. Unfortunately, windows 
applications are still utilized in many businesses as tools for group work. This research made the attempt to identify accessibility and usability issues in regard to windows-based applications, specifically Microsoft Outlook. Accessibility and usability issues occurred while reading text messages, or reading the wrong message, accessing items in the calendar, logging in, and accessing the journal and contacts.

The key usability issues were related to the steep learning curve and the inability to solve the accessibility issues encountered. These issues were identified as the main reasons why persons who are blind discontinued use or would not attempt to utilize new collaborative applications. Even though all of the participants of the focus group study were capable users of the operating system, the selected collaborative applications, and screen access software, they still expressed the apprehension of utilizing new collaborative applications. Given the participants' skill levels and their difficulties with inaccessible collaborative applications, it can be predicted that less skilled persons who are blind may experience more severe challenges when interacting with collaborative applications. This may be more serve of a challenge without accessibility support and working in a group with sighted persons.

The third and final research question "What kind of support do persons who are blind require so that they can perform group work?" was answered during the pilot focus group study. While conducting the group process, persons who are blind will utilize adaptive technologies such as: screen readers for reading documents and email messages; and, note taking devices to record minutes during meetings. Similarly, Woodfine (2008) identified the need for special support and assistive tools for persons with dyslexia to 
interact in synchronous e-learning environments. How can we better integrate these adaptive technologies into the group process for the support of group work?

Takagi's (2000) Notes Reader and Watanabe's (2007) Blog Reader were two examples of redesigning the web page interface and including voice-over as feedback. Given this prior research, a design suggestion of an accessible self-voicing, or voice-over, application was presented as an alternative to utilizing a screen reader. Even though Takagi's (2000) and Watanabe's (2007) voice-over solutions worked in their case, it was determined by this research to be a less-acceptable solution to the use of a screen reader as the interface to collaborative applications. Therefore, it is important to consider screen readers and their capabilities whenever developing applications that are accessible and usable, since screen reader support is critical for persons who are blind.

The focus group study participants expressed the need for consistency, when discussing design considerations. Similarly, Luk (2000) presented spatial clustering as a method to organize content and make the interface consistent. For the most part, the focus group participants agreed that the Microsoft Outlook interface was good, but the interaction between the screen reader and the application was the primary cause of their accessibility issues. As different versions of the screen reader software are developed, older versions of collaborative applications may no longer be accessible. Since consistency between versions of collaborative software and screen reader software are important to accessibility and usability, how can researchers and developers deliver consistent and integrated applications that function properly with screen reader software?

Unique to this research is the presentation of the concept of determining the appropriate level of accessibility support and documentation needed by persons who are 
blind. Often, the participants of the focus group want to use new features of collaborative applications or even new applications, but the support and the documentation on accessibility is limited or non-existent. This lack of support for accessibility causes frustration on the part of persons with disabilities and their discontinued use of the application. Fortunately, for the Access Technology and Education Team at the NFB, they are the support team devoted to accessibility and usability. However, in other environments this level of support may not be available.

\subsection{Conclusion}

The findings of this research included identifying the collaborative applications utilized by the participants, understanding the group process, identifying accessibility and usability issues when utilizing a screen reader in conjunction with collaborative applications, and the type of support needed to make collaborative applications accessible and usable.

The pilot focus group study participants identified Microsoft Outlook as their primary collaborative application for message exchanging, task tracking and appointment scheduling. However, the participants did not identify any groupware application used for collaboration. Further research with additional participants will be necessary to determine if groupware is utilized, or that they too utilize collaborative applications and not fullfledged groupware.

The participants reported that Microsoft Outlook has accessibility and usability issues. For example, while parsing a list of messages in the "In Box" of Microsoft Outlook, the JAWS screen reader read the wrong email message or did not read the email message at all. 
Consequently, the participants were concerned that they may miss important messages that need immediate response. How can end users be certain they are reading the selected or intended message properly? How can they determine the number of messages or range of message to make certain they have not overlooked an important message? Another concern presented by the participants, included the Microsoft Outlook login screen randomly displaying when the network connection was lost. Unfortunately, the JAWS screen reader did not recognize the random events. In other instances, the JAWS screen reader did not properly read the login screen content. Not recognizing random login events or not properly reading the login screen prevents persons who are blind access to Microsoft Outlook content causing interrupted service. Consequently, resulting in missed messages, meetings and lost time trying to regain access to Microsoft Outlook. How can random events such as displaying the login screen be recognized by the JAWS screen reader? Can the JAWS screen reader access some system-level service to determine if an application has lost its connection or other random failure has occurred?

As far as the Microsoft Outlook interface is concerned, the focus group study participants' reported that they modified Microsoft Outlook's interface by reducing the panels displayed. As a result of this adjustment to the Outlook panels the content of the interface was reduced in turn reducing the content read by the JAWS screen reader.

Consequently, reducing the content read by the JAWS screen reader helps avoid overwhelming the end user with too much content. How much text a screen reader can present before persons who are blind are overwhelmed? Is there a method for controlling the amount of text spoken by the JAWS screen reader? 
All of the findings point to the need to improve the operation between the applications' interfaces and the screen reader. Controlling the amount of content presented and recognizing random events are critical to successful interactions and end user understanding.

In addition to identifying findings and future research questions, the purpose of the pilot focus group study was to validate the research instruments utilized to make sure that the set of data collected was what was needed and to determine if there were any ambiguous questions to reword or new questions to add to the research guideline. The research instruments seemed appropriate for this descriptive focus group study. The online survey used to collect demographical data was appropriate for the participants since it was an accessible survey tool. The audio recording proved vital in recording exactly the questions and responses during the focus group study. The audio recording helped in reconciling the notes taken by the researchers. The grounded theory and the use of Cohen's Kappa proved ideal for coding and validating the data collected. The focus group study research guideline included key questions for the three general areas we were attempting to gather data. Questions in regard to demographics and computer experience were added to the research guideline, since the survey/questionnaire for this information was post-study in the main focus group studies and the participants may not respond.

Since the pilot focus group study uncovered the fact that modification of the interface was necessary in some cases to make the interface accessible and usable, a question was added to explore this concept with the main focus group studies to determine if this was the norm. 
The limitations of this preliminary focus group study are due to the small number of participants and the common backgrounds of the participants. Therefore, the results cannot be generalized.

At this point, Microsoft Outlook contains accessibility and usability issues, the group process has been documented, and the reasons for discontinued use are known. Additional field studies and focus group studies are necessary to gain a complete picture of the accessibility and usability issues involving collaborative technology.

The next step in this research is to gain more in-depth information about the problems identified and broaden the scope, by conducting field studies to observe how persons who are blind utilize the collaborative technology features. 


\section{FIELD STUDIES}

\subsection{Introduction}

Following the pilot focus group studies, two field studies were conducted at the National Federation of the Blind (NFB) (2008), in Baltimore, Maryland, in the summer of 2010. The field studies were undertaken as a method to verify the pilot focus group study results. The purpose of these studies was to understand how persons who are blind work in different types of groups (co-located or distributed, synchronous or asynchronous) (Table 1), the collaboration support they require in each scenario, the group process they follow, and any accessibility and usability issues they encounter. Additionally, the focus group studies were undertaken to examine the group processes and experiences with collaborative applications from a broadened group of participants.

During the previously reported pilot focus group study, the group project process and the accessibility and usability issues were identified and later utilized as a roadmap of the tasks to observe during the field studies. Several participants of pilot focus group study were recruited for participation in the field studies conducted at the NFB, so that continuity between the pilot focus group study and the field studies could be maintained and the content provided during the of the pilot focus group study could be validated. 
The following research questions were addressed during this study:

- RQ1: How do persons who are blind work in groups including different scenarios?

- RQ2: What is the accessibility and usability challenges persons who are blind experience with software applications (specifically collaborative systems)?

- RQ3: What kind of support do persons who are blind require so that they can perform group work?

\subsection{Research Methods and Procedures}

Field studies are observations of study participants as they interact in their environment (Burgess, 1995) and present many benefits. The environment and the participants can be observed in their actual settings or work environments, allowing researchers to study situations from the participants' point-of-view. Often, field studies are used to validate up-to-date data and information provided by surveys and focus group studies. Field studies can be utilized to identify and prioritize their needs, and provide clarity by revealing what you do not know from prior data and information and opportunity for new data and information ("Niesen Norman Group Seminar," 2011; "Field Research," 2011; Burgess, 1995). Field studies are well suited for validating focus group study data and information and to collect any gaps in the data or information.

Field studies also present some limitations. Often, the qualitative methods of field studies are considered to be soft, subjective and speculative and the participants' behavior may not be candid. The participants may provide only positive interactions or they may provide some but not all interactions normal to a process. Researcher's observations may be misinterpreted by small sample sizes that take time and are expensive. Even under the best circumstances, field studies may omit some activities or the activities may be 
overlooked completely by the observers ("Niesen Norman Group Seminar," 2011; "Field Research,” 2011; Burgess, 1995). These limitations should be considered and multiple observations by multiple observers should be undertaken in order to gain a full understanding of the participants' interactions.

The field study sessions, contained the following steps:

1. Recruit the participants;

2. Prepare demographic, prior-experience questionnaire and meeting guideline;

3. Acquire participant consent;

4. Conduct the field study session;

5. Perform content analysis of the audio transcript and the researcher notes, to identify content categories;

6. Validate agreement of the coded content, using Cohen's Kappa (2010); and,

7. Report findings.

The field studies' sessions started with a brief introduction from the researcher on the purpose of the studies, followed by observations of the participants for approximately one-hour as they performed their projects' tasks. 
The focus of the observations was based on the following situations and scenarios:

- What collaborative applications and other tools did the participants utilize during the group project process?

- Where (i.e. in the office, in a conference room, etc.) do the participants utilize collaborative applications and other tools?

- For what tasks (i.e. email, document sharing, etc.) do the participants utilize collaborative applications and other tools?

- What accessibility and usability issue do the participants encounter?

Various collaborations were observed that were based on the time-place

framework from Table 1. For instance, co-located group meeting in a conference room to discuss project plans, and exchanging email messages and sharing documents with virtual/co-located groups

After the observation period, follow-up probing questions were asked and audio recordings were captured, to ensure the understanding of the field studies' observations. The audio transcripts and detailed notes taken during the field studies were coded based on the themes that emerge from the participants' comments. 
6.3 Results from the Field Studies

\subsubsection{Demographics}

The participants of the field studies included members of the NFB. The participants consisted of one female and one male. The two participants are between 20 and 39 years old. All of the participants utilized the computer daily, for business use, and have used the computer between 11 and 20 years. All participants utilize the JAWS Screen Reader ("Freedom Scientific JAWS," 2008) with participants having 6 to 10 years' experience. Collectively, all participants of the field studies are experienced with the Windows Operating System, the JAWS Screen Reader, and interacting with Microsoft Outlook and other tools (TIKI WIKI, AOL IM, Microsoft Word, Microsoft Excel), when conducting group projects. The high-experience level of the field study participants can establish the best case examples when researching accessibility and usability, screen reader utilization, collaboration experiences and challenges, and identifying areas for improvements.

\subsubsection{Content Analysis of the Field Studies' Recordings}

Content analysis was performed on the audio transcriptions and notes collected during the field studies, the common themes or categories with their associated annotated list elements are shown in Table 14. 
Table 14. Categories and Annotated List Elements

\begin{tabular}{|l|l|}
\hline \multicolumn{1}{|c|}{ Category } & \multicolumn{1}{c|}{ Annotated List Element } \\
\hline $\begin{array}{l}\text { 1. Participant's identification of } \\
\text { collaborative software utilized }\end{array}$ & $\begin{array}{l}\text { a. Features of collaborative } \\
\text { applications utilized; } \\
\text { b. Other applications utilized; and, } \\
\text { c. Applications utilized when } \\
\text { conducting group projects. }\end{array}$ \\
\hline $\begin{array}{l}\text { 2. Tasks/steps necessary to complete group } \\
\text { projects }\end{array}$ & $\begin{array}{l}\text { a. Frequency of group meetings; and, } \\
\text { b. Tasks necessary to complete a } \\
\text { group project. }\end{array}$ \\
\hline 3. Accessibility and usability issues & $\begin{array}{l}\text { a. Accessibility issues encountered } \\
\text { when using collaborative } \\
\text { applications; }\end{array}$ \\
& $\begin{array}{l}\text { b. Issues encountered when trying to } \\
\text { utilize new applications; and, }\end{array}$ \\
& $\begin{array}{l}\text { c. Issues encountered when using the } \\
\text { JAWS Screen Reader. }\end{array}$ \\
\hline 4. Group interaction techniques & $\begin{array}{l}\text { Techniques used for group } \\
\text { interactions. }\end{array}$ \\
\hline 5. Group projects & Group project types \\
\hline 6. Accessibility design considerations & $\begin{array}{l}\text { a. Proposed solutions for issues } \\
\text { encountered; and, } \\
\text { b. Modification of the interface. }\end{array}$ \\
\hline 7. Accessibility documentation and support & Accessibility documentation. \\
\hline 8. Collaborative applications' benefits & $\begin{array}{l}\text { Benefits of utilizing collaborative } \\
\text { applications. }\end{array}$ \\
\hline
\end{tabular}


Eight categories were developed from the annotated list as follows:

1. Participants identification of collaborative software utilized;

2. The tasks/steps necessary to complete a group project;

3. Accessibility and usability issues;

4. Group interaction techniques;

5. Group projects;

6. Accessibility documentation and support;

7. Accessibility design considerations; and,

8. Collaborative applications' benefits.

The Cohen's Kappa measurement of agreement sought was $70 \%$ or greater, to provide evidence of acceptable agreement and reliability between the coders. As a result of combining the field studies' coding data there was a total of 127 cases identified and the agreement between the two coders for both field studies was $86.9 \%$. This agreement provided evidence of an acceptable agreement and reliability level between the coders. The following sub-sections will present each of the eight categories identified with discussions.

\subsubsection{Participants identification of collaborative software utilized}

Critical to this research was the identification of the collaborative applications and the adaptive technologies utilized by the field studies' participants, in order to determine if the applications are accessible and what tools are utilized to access these applications.

Collaborative applications observed being utilized by the participants included the following: Microsoft Outlook, for various email, journal, calendar, contacts, and tasks, 
activities which support distributed-asynchronous communications (Table 1); AOL Instant Messenger, for spontaneous messaging in an informal manner providing distributed-synchronous communications support (Table 1); Microsoft Word for shared document editing and commenting representative of distributed-asynchronous collaborations (Table 1); and, TIKI WIKI (2013) for document storing, sharing and exchanging over the Internet as distributed-asynchronous collaborations (Table 1), Braille Sense, for group meeting note taking; and, Microsoft Excel, for analytical operations. Features (email, track document changes, Chat, and shared data storage) of collaborative applications were observed; however, the participants did not utilize traditional groupware applications during the field studies. Therefore, the focus of this study was on collaborative applications, which included some features of groupware and not on traditional groupware applications.

The participants in their individual offices utilize the collaborative applications, such as, Microsoft Outlook, TIKI WIKI, and AOL IM. The participants of the field studies on a daily basis utilize Microsoft Outlook, Excel and Word and on an informal basis they utilize AOL IM. The WIKI, TIKI WIKI, is currently in a participation "buyin" phase, but there are some noticed benefits to the WIKI. A participant of the field studies commented, "We do a lot of training offsite, so we have a situation when only one person had access to all the information and she was unavailable and did not share the information. We could use the WIKI in the future and not have to rely on one person and try to track them down. The WIKI will cut down on redundancy and will be easier to share a common document. In the WIKI, you can track the history of a document. The 
documentation is on line and we had the WIKI software placed on our local server. The main problem now is to get buy-in from the other associates."

The TIKI WIKI application was a new application identified since the pilot focus group study occurred. The purpose of the TIKI WIKI is to facilitate a knowledge base of documentation and project plans that can be accessed via the Internet. The use of the TIKI WIKI can improve the productivity of the group members at the NFB, by reducing the number of copies of a document, providing remote access to a document from the Internet, providing a single place to store and retrieve documents, and providing document sharing. Additionally, Microsoft Word and Microsoft Excel were identified as the specific tools for document sharing and spreadsheet sharing within the group.

During the field studies, observing the participants as they interacted with these applications helped identify the collaborative applications and the adaptive technologies utilized. These types of observations become more valuable than focus group conversations, since they are true observable and validated events.

\subsubsection{The Tasks/Steps Necessary to Complete a Group Project}

The purpose in identifying the tasks or steps necessary to complete and group project is to provide a roadmap for observations during the field studies. In addition to understanding the collaborative applications utilized, it was important to observe and identify the tasks or steps utilized when completing a group project. The steps reported by the participants and observed by the researcher include the following: face-to-face meetings; note taking by someone during the meeting, using a Braille Sense note taking device; documentation of the meeting and plans are written; emails containing the 
meetings' content are exchanged; and, a work plan is developed. A participant of the field studies commented on the typical project flow, "Internally, we have a lot of face-to-face meetings. We do conference calls with external groups we work with. We start with faceto-face meetings and someone taking notes. Projects generally start with face-to-face meetings. Then, we write documents, shoot emails and a work plan is developed. Then, a meeting is arranged to go over the work plan"

Additional meetings are conducted to review the work plan. Once the work plan is emailed, several versions of the work plan can exist. According to a participant of a field study, "The work plan is generally emailed out to all participants. We have about 40 copies floating around. We have a shared network drive, but not everything ends up on the server. That is why we are trying to use the WIKI."

Observing the project steps, we have identified the steps for completing a group project, the technologies (Microsoft Outlook, Microsoft Word, and TIKI WIKI) that are necessary for the completion of a group project; and, the adaptive technologies (Braille Sense Note taker and JAWS Screen Reader) utilized to access these applications. At this point the tasks or steps to completing a group project are known, providing a roadmap for observations.

\subsubsection{Accessibility and Usability Issues Observed/Discussed}

A significant purpose for this field study was to determine what accessibility and usability challenges exist. During the field studies, the researcher observed actual accessibility and usability issues while the participants interacted with collaborative 
applications. These observations of the accessibility and usability issues provide opportunities for further research and the development of solutions for these issues.

\subsection{Microsoft Outlook Issues}

The participants of the field studies reported accessibility and usability issues with several of the features provided by Microsoft Outlook. A feature of Microsoft Outlook, that was considered to be inaccessible by the participants of the field studies, was the Outlook Calendar Month-view. The Outlook Calendar Month-view presents an entire month, in a typical two-dimensional monthly-calendar format. In regard to the accessibility of the Outlook Calendar Month-view, a participant commented, "I don't have a lot of problems with the Calendar. I still can't work with month-view. You are limited to day-view. The month-view looks more like a calendar and you don't have access to the time-slots like with the day-view."

The ability to transfer directly from a calendar month-view to a drilled-down detail view, such as the day-view, may make the calendar month-view more accessible and usable. A key press sequence may be one option to signal the transfer from monthview to day-view. Another participant commented, "I generally use control-g (go to) and type in a date, to access the calendar."

Another feature of Microsoft Outlook, that was considered to be inaccessible by the participants of the field studies, was the Outlook Login Message Box. The login message box can randomly display and causes the JAWS Screen Reader to stop speaking. The participant had to uncheck the working offline menu item option within Microsoft Outlook's Application, to receive a new login message box that the JAWS Screen Reader could read. The participant logged back into Microsoft Outlook. After this event 
occurred, the participant commented, "For me, it happens when the Outlook Session is open for a while and Outlook decides it has to re-authenticate. It keeps insisting I am offline, when I am not. I can't pick out a cause. Usually hitting the escape key on the keyboard and uncheck the work offline checkbox resets everything. Escape puts you in offline mode, then you can recheck the work offline checkbox and re-authenticate."

Notifications of certain events (offline, login, etc.) are necessary for end-users' awareness and to allow them to properly interact with the application. A method of notifications of certain events is a design consideration of an accessible and usable application interface. For persons who are blind, auditory notifications may be the best approach.

The participants of the field studies do not utilize the Outlook Journal, due to accessibility and usability issues. The participants discussed the inability of determining the cursor position within the Journal. A participant of the field studies commented, "Time-line view is not accessible. Not a lot of people use the journal. I do not understand where I am in the Journal. I do not understand the cursor position within the Journal."

Determining the cursor position may be a common issue in regard to any list of items, such as, the list of email messages, the list of journal entries, and the list of tasks. Providing auditory clues for cursor position to support orientation of the interface may be an important interface design consideration. The screen reader reading the interface components should provide the cursor position and the auditory clues, to make this a complete solution for orienting lists. 


\subsection{TIKI WIKI Issues}

The participants of the field studies reported accessibility and usability issues with features of TIKI WIKI. While discussing and observing TIKI WIKI, the several controls on the TIKI WIKI interface were considered to be inaccessible by the participants of the field studies. A participant commented, "Things (controls) don't have labels tied to them the way they should. So, check boxes usually have the label to the right of the check box; the TIKI WIKI has the label to the left. The checkbox is usually tied to the label." Improperly labeled controls of the interface such as the case with the TIKI WIKI checkbox controls cause accessibility and usability issues for persons who are blind. Specifically, the screen reader is dependent on properly labeled controls in order to access and read the underlying content. Consequently, the screen reader will not properly read the content of the interface and access to this content will be unavailable. Therefore, properly labeled controls (text boxes, radio buttons, and check boxes) are necessary for persons who are blind to interact with the interface while using a screen reader.

\subsection{Screen Reader Interactions' Issues}

The participants of the field studies reported accessibility and usability issues while interacting with their applications using the JAWS Screen Reader. When the screen reader is overloaded with too much textural information, auditory information overload can overwhelm persons relying on the screen reader for application interaction. When using Microsoft Word's editing features such as track changes, a participant of the field studies commented, "The JAWS Screen Reader gets cluttered with the changes. It doesn't present auditory as it does visually. There are ways around the track changes, where you 
can get a list of all the changes." If the JAWS Screen Reader is reading a track change, then it should not lose focus and move to a new track change until the end-user has heard the entire change, or has stopped the reading of a tracked change. Reviewing the track changes as a list at the end of a document may provide a mechanism to scroll through the list of changes. However, Microsoft Word has to control the presentation of the textural information, so not too many changes are presented to the screen reader at one time, causing the auditory overload.

After upgrading the JAWS Screen Reader Software, some applications are no longer accessible. A field study participant commented, "The issues are with the (Microsoft Excel) verbosity settings. You were able to set row headings, and save your settings. Sometimes it will save your headings and sometimes it won't. An Associate in our Accessibility and Education Team discovered a work-around, where you define your regions, and that has seemed to work." Whenever new software is developed, in this case a new JAWS Screen Reader, the interface should be validated and verified that it continues to properly interface any know applications, as expected and new issues are not introduced. This may be a tough item to fulfill, since there are a huge variety of applications that the screen reader has to interface. In this case, a coordinated effort between the screen reader developers and some organization, such as the NFB, that represents the target market of end-users may be necessary.

The observed accessibility and usability issues of Microsoft Outlook, TIKI WIKI, and Microsoft Word all provide future opportunities to correct any of these issues. At this point, there are accessibility and usability issues that require further investigation and solution development. 


\subsubsection{Group interaction techniques}

Group interaction techniques can provide how the group interacts with each other while communicating. The important thing to consider is do the team members utilize the best technologies (email, text messaging, document sharing) available or do they utilize less sophisticated techniques (telephone, hand-written notes), to communicate? And if they utilize the latter, is it due to accessibility and usability issues or the apprehension of using unfamiliar technologies?

The field studies' participants were observed communicating as a group by utilizing collaborative applications for document sharing, document change tracking, document retaining, messaging, calendaring, and project tracking. The field studies' participants reported and were observed communicating in face-to-face meetings and teleconferences. One participant commented, "We have a series of emails leading up to our face-to-face meeting. We schedule a meeting. Everything is calendared. But then, when we have our actual meeting, we have a lot of conference rooms and the meetings are face-to-face. If we do have external people, we have a conference line."

During the conference room meeting, a participant was observed using a note taker to record and document the meetings' content. Then, the meetings' content can be uploaded to a server for group sharing. The observed participant commented, "I usually take notes with my Braille Sense. I connect my Braille Sense to my computer and I can just copy the file." The Braille Sense may be the device to move content from meetings to electronic form for document sharing by any group.

During these conference room observations, it was discovered that even though there may be accessibility and usability issues with collaborative applications, the 
participants were able to work-around these issues. For example, while working with Microsoft Word, a participant could not follow the track changes, so the participant simply accepted all of the changes. Once all of the changes were accepted, the participant could review the entire document. This approach is not optimal, but is a temporary solution. What is needed is a better approach to presenting changes to document that persons who are blind can properly access and use.

\subsubsection{Group Projects}

The participants of the field studies reported that they participate in various projects. Some of the projects include: the Junior Science Academy; accessibility, training; and, education. The information retained in regard to these projects includes: student contacts, lesson plans, lesson time frames, and science projects for simple machines (pulleys, wedges, and levers). A participant commented, "We use the WIKI for programs like the Junior Science Academy. We have materials out there. So, we keep adding items to the materials list, the Parents' seminars and the kids' seminars."

\subsubsection{Accessibility design considerations}

The purpose of understanding the design considerations of a collaborative application's interface is to incorporate the necessary features into the interface so the application is accessible and usable. What interface requirements do persons who are blind need in order for the application to be accessible and usable? One of the interface requirements may be to be accessible and usable with a screen reader. Simplifying the interface by grouping common items and presenting small lists of available options are a 
few possibilities. Understanding these requirements is important to providing accessible and usable collaborative applications.

Customizing/modifying the applications' interface is one approach to enhancing accessibility and usability. During the field studies, several modifications to the Microsoft Outlook application's interface were observed. For example, the messages, calendar and contacts were all displayed as lists, with the removal of other interface panels and hierarchy trees.

A participant commented, "I just get annoyed by the extra stuff. When I tab, it goes through the extra stuff in the tree-view. I just like to get at what I need. I don't need that cluttering up the interface. Since there are other ways to get at what I need, I do not need the tree-view cluttering up the interface. Particularity with the Inbox, it cuts down on the amount of information read. With the tree-view removed from the interface, I get the subject and a shortened message. Since the interface cuts off the message in the expanded view, there is less message text to read." Even though the folders are not visible on the interface, access to the folders is obtained by a key-press combination (control-shift-v). Another participant commented, "There is actually a way to move the email message without actually bringing up the folders. It is a quick command. If I want to go to a folder I enter, control-shift-v, select the folder, and then enter will move the message to the folder and return you to the messages."

The ability to modify the interface to be less cluttered is a feature of applications that is necessary for improved accessibility and usability. The screen reader can preview the less cluttered interface with only the messages as a list without the additional clutter 
(hierarchy list of folders, and the message preview panel) on the interface normally available with Microsoft Outlook.

Another approach that helps make an interface easily accessible is to utilize access-key press combinations to navigate. Several examples of utilizing access key to navigate or directly search for content. A participant of the field studies commented, "I use the hot-keys. If I don't know where it is in the menus, I hot-key to the main menu Then, I search the list." Another participant commented, "I generally use control-g (go to) and type in a date, to access the (Microsoft Outlook) calendar."

Navigation of menus can be accomplished by using arrow-keys and alt-key press combinations. A participant commented, "I usually search the menus. I go across the top, using the arrow-keys. I usually forget the alt-key combinations, so I just alt-key then, I search the menus."

Many applications can be accessible to persons who are blind by providing keypress combinations for direct access. When accessing the menu of most applications, keypress combinations allow for direct manipulation of the menu and allow for direct access to features of the application.

A less cluttered, key-press accessible, and screen reader accessible interface is necessary for collaborative applications to be accessible and usable for persons who are blind. 


\subsubsection{Suggestions on accessibility documentation and support}

The field studies' participants identified the importance of having access to documentation of accessibility features of collaborative applications. Documentation on accessibility is necessary, so persons who are blind can make informed decisions in regard to what accessibility features they wish to use. For example, documentation for voice-over within an application, so that persons interacting with the application can utilize the voice-over feature or disable the voice-over feature and use a screen reader they may prefer. A participant of the field studies commented, "Part of the problem is you are not using the application to interact with the application; you are using the screen reader to interact with the application. Have the application developers document whatever accessibility features they have built into the application, so we are aware of the features."

Windows provides accessibility features can be utilized and there are free screen readers available. A participant commented, "Sometimes it is important to know accessibility features in Windows, when a parent tells me they cannot afford a screen reader for their child. Then, in that instance it is important to know other accessibility features. Alternatively, System Access to Go (SATOGO) is a program for a free reader. It works pretty well." Additionally, state employment agencies will often pay for screen readers.

Additionally, the participants have the support of the Access Technology and Education Team for issues in accessibility and usability. A participant commented, "I do not usually use the documentation with JAWS, because I can't find what I want. Usually, 
I will call the Access Technology Team. They are in the know - it is usually faster." Persons who are blind that do not have the support of an accessibility team are more likely to rely greatly on accessibility documentation as their only source of support. It can be concluded that accessibility documentation and support are important features that should be included with applications. The benefits of accessibility documentation are to enable awareness of accessibility features, so they may be utilized under the proper circumstances.

\subsubsection{Collaborative Applications' Benefits}

Understanding the benefits of collaborative applications may help to entice persons who are blind to utilize these applications when performing group work. The benefits of utilizing these applications can include: virtual meetings, with persons at multiple locations; message sharing, through the use of email applications; document sharing and co-editing, through the use of change tracking document applications; and, calendar sharing, through a calendar application. These are a few of the benefits of utilizing collaborative applications. A participant commented," "We have about 40 copies (of a document) floating around. We have a shared network drive, but not everything ends up on the server. That is why we are trying to use the WIKI. The WIKI will cut down on the redundancy and will be easier to share a document."

Even though there are benefits to utilizing collaborative applications, often these benefits are not realized when the document changes are not accessible or usable. A participant commented on the accessibility and usability issues with track changes features of some document sharing applications, "Sometimes we (persons who are blind) 
edit the documents on the server, and because the sighted people will do track changes, but there are issues because JAWS cannot read those. If they (sighted people) proof a document and say something is wrong, we (persons who are blind) can't approve those changes. We (persons who are blind) have a support team to help us with the track changes. So, if you have track changes, they are not accessible."

\subsection{Discussion}

The participants of the field studies are experienced in utilizing windows, the JAWS Screen Reader, and various collaborative applications. Additional studies are needed with persons with diverse backgrounds of experience.

The field studies uncovered a new application utilized at the NFB, to support group collaboration. The new application, TIKI WIKI, has promise in facilitating project tracking and document sharing over the Internet. The reduction of multiple copies of documents and the ability to access the WIKI from any location are the benefits of utilizing this technology.

The tasks necessary to complete a group project at the NFB were validated during the field studies. The note taking device, Braille Sense, was identified for taking notes at face-to-face meetings. After the face-to-face meetings, the notes recorded on the Braille Sense are imported into the Computer as formatted Microsoft Word documents. During the pilot focus group study, the note-taking device was not identified, so this bit of information helps present the entire group meeting process and the technology utilized.

The applications, Microsoft Outlook, TIKI WIKI, and Microsoft Word's track changes feature, are all determined to be inaccessible and unusable to a certain degree. Whenever, any application is modified or enhanced by new releases of the application, 
accessibility and usability should be considered when validating test results.

Unfortunately, it is suggested by the participants of the field studies that as new applications are developed, they are not tested for accessibility and usability.

The participants from the field studies participate on group projects of various types. Collaborative applications are an integral part of these group projects. Therefore, accessible and usable collaborative applications are necessary for persons who are blind to function as group members.

In regard to accessible design considerations, the field studies participants prefer utilizing a screen reader instead of utilizing an application that includes voice-over. The issues of conflicting audio content and the additional learning curve were reasons for their preference. The ability to modify the interface, by removing unnecessary content, such as: panels and folders, is an important feature for an accessible interface. According to the participants of the field studies, the availability of access-keys or short-cut keys is preferred to access menu items over the use of a ribbon-based menu structure.

The access to accessibility documentation for collaborative applications is needed for the purpose of being aware of the accessibility features, so they may be activated and deactivated as needed. It is important to know what accessibility features are available in order to avoid conflicts while utilizing screen access software, such as: screen readers. Unfortunately, according to the field studies participants, accessibility features within these applications are not documented, or are difficult to locate.

The participants of the field studies identified several benefits of utilizing accessible and usable collaborative applications. However, the field studies participants 
indicated that these benefits are not realized, if these applications are not accessible and usable. 


\subsection{Conclusion}

The field studies provided observable evidence supporting the data collected during the pilot focus group study with the Access Technology and Education Team. The findings of the field studies' research included findings similar to the pilot focus group study including identifying the collaborative applications and other applications utilized by the participants, identifying specific examples of the group process, identifying accessibility and usability issues when utilizing a screen reader in conjunction with collaborative applications, and identifying the type of support needed to make collaborative applications accessible and usable.

The participants of the field studies were observed interacting with Microsoft Outlook, TIKI WIKI, and Microsoft Word's track changes feature in conjunction with the JAWS screen reader. These observed applications supported the notion that the Access Technology and Education Team did not utilize full-fledged groupware, but collaborative applications were utilized instead. During the pilot focus group study and field studies, Microsoft Outlook was identified as the primary collaborative application for message exchange, task tracking and appointment scheduling. Microsoft Outlook, TIKI WIKI and Microsoft Word all presented some accessibility and usability challenges.

The group process was observed during the field studies to determine the tasks and the support utilized for each task. The types of tasks supported by collaborative technologies included message exchange, document co-editing and storage, remote data access and storage, note taking, etc.

Accessibility and usability issues were identified while utilizing Microsoft Outlook. The login message box randomly displayed whenever Outlook lost a 
connection. The JAWS screen reader did not recognize this random event. Consequently, access to the application is denied. How can a screen reader recognize random events? In many instances, random events are displayed within dialog boxes that are graphical. Is there another way other than displaying a dialog box to notify screen readers of random events? Alternatively, the applications could send a signal to the screen reader that a random event is about to occur. Whether a screen reader could interpret an event signal would have to be investigated. The journal timeline was difficult to access and utilize, since the timeline is presented as a horizontal list of dates that the screen reader reads consecutively. There is no real separation of dates to allow specific date selection. Consequently, access to the journal entries for a specific date is not possible. Therefore, the participants of this research do not utilize the journal. Similarly to the journal, the calendar month-view was not accessible. The participants could not correctly identify and select a specific date on the calendar interface either. Therefore, access to a specific date is not possible and the content would be unavailable. Unfortunately, missing the content of a specific date could impact the missing of important deadlines. Alternatively, the field studies' participants switched to the calendar's daily-view in order to access the content for a specific date. How can complex interfaces with multiple cells/dates in a grid pattern be presented so a screen reader can distinguish the cell content and its position in the grid? Alternatively, should grid structures be avoided when designing interfaces that are designed to be accessible and usable? What other interface structure could be utilized to represent a series of cells/dates?

Accessibility and usability issues were identified while utilizing TIKI WIKI. The controls on the interface were not properly labeled, causing the screen reader to ignore 
certain content. Consequently, participation is hindered by not being able to access improperly labeled content. Therefore, participant contributions would be omitted that may be of value.

The JAWS screen reader had some issues while reading text, which overwhelms the end user. This issue was identified when the interacting in a Chat session with a screen reader. As the screen reader reads the text received, there is not sufficient time for persons who are blind to respond before being overwhelmed by additional text being read. Consequently, participation in Chat sessions is not possible. How can the text presented during a Chat session be limited or controlled, so not to overwhelm the end user? How much text can a screen read present before the end user is overwhelmed by the content?

Tracked changes in a Microsoft Word document were difficult to understand by the field studies' participants. The screen reader read the tracked changes that seemed garbled to the participants. Consequently, the participants could not understand the changes made to the word document. As a result, the tracked changes were all accepted and then read by the screen reader, so the participants could review the changes. How can tracked changes in a Microsoft Word document be presented to a screen reader that will result in content that is understandable?

All of the findings from the pilot focus group study and the field studies point to the need to improve the interaction between the screen reader and collaborative and other applications. What messages or content are presented, how much content is presented, and how understandable is the content are key to accessibility and usability. Additionally, 
handling random events is crucial to successful interactions between any application and a screen reader.

Conducting additional focus group studies with additional participants should help collect more data representing the accessibility and usability issues persons who are blind experience. The NFB State Convention was such a venue where potential participants were available to participate in the additional focus group studies. 


\section{ADDITIONAL FOCUS GROUP STUDIES}

\subsection{Introduction}

Three additional focus group studies were conducted at the State Convention of the National Federation of the Blind (NFB) (2008), in Annapolis, Maryland, in the fall of 2010. The purpose of the focus group studies was to expand on the data and information collected from the pilot focus group study and the field studies presented earlier. Each focus group study included between 4 and 5 participants in order to provide all of the participant's ample time to contribute to the conversations. All of the participants of the pilot focus group study and the field studies are actual group members at the NFB in Baltimore while all of the participants of the focus group studies at the State Convention are from throughout Maryland and are not group members on the same team.

In order to be easily accessible for the participants, the focus group sessions were conducted in a private conference room at the NFB State Convention. The researcher worked as a facilitator in the focus group studies, which ran for approximately one hour in duration. A meeting guideline (Appendix B) from the pilot focus group study was utilized to present questions for discussion. The audio of the group discussion was recorded and later transcribed with the permission from the participants. Meanwhile, the moderator also took notes during the discussion. Both transcript and detailed notes on the group discussion were coded based on the themes that emerge from the participants' comments. 
The common themes or categories with their associated annotated list elements are shown in Table 15.

Table 15. Category and Annotated List Element

\begin{tabular}{|c|c|}
\hline Category & Annotated List Element \\
\hline $\begin{array}{l}\text { 1. Participant's identification of } \\
\text { collaborative software utilized }\end{array}$ & $\begin{array}{l}\text { a. Features of collaborative } \\
\text { applications utilized; } \\
\text { b. Other applications utilized; and, } \\
\text { c. Applications utilized when } \\
\text { conducting group projects. }\end{array}$ \\
\hline $\begin{array}{l}\text { 2. Tasks/steps necessary to complete group } \\
\text { projects }\end{array}$ & $\begin{array}{l}\text { a. Frequency of group meetings; and, } \\
\text { b. Tasks necessary to complete a } \\
\text { group project. }\end{array}$ \\
\hline 3. Accessibility and usability issues & $\begin{array}{l}\text { a. Accessibility issues encountered } \\
\text { when using collaborative } \\
\text { applications; } \\
\text { b. Issues encountered when trying to } \\
\text { utilize new applications; and, } \\
\text { c. Issues encountered when using the } \\
\text { JAWS Screen Reader. }\end{array}$ \\
\hline 4. Group interaction techniques & $\begin{array}{l}\text { Techniques used for group } \\
\text { interactions. }\end{array}$ \\
\hline 5. Accessibility design considerations & $\begin{array}{l}\text { a. Proposed solutions for issues } \\
\text { encountered; and, } \\
\text { b. Modification of the interface. }\end{array}$ \\
\hline 6. Accessibility documentation and support & Accessibility documentation. \\
\hline
\end{tabular}

Agreement among coders was calculated using SSPS (“SSPS Inc.," 2010), using Cohen's Kappa (2010). The Kappa measurement established for measuring agreement between coders was $70 \%$ or greater agreement. 94 cases were identified with the coders agreeing on $97 \%$ of these cases, providing evidence of acceptable agreement and reliability between the coders. 
7.2 Results from the Focus Group Studies

\subsubsection{Demographics and Experience}

The participants of the focus group studies included fourteen participants, consisting of nine females and five males, attending the NFB State Convention. Of the fourteen participants, nine participants completed the demographical and experience survey distributed online. Computer experience was discussed during focus group; therefore, the analysis for this factor was based on all fourteen participants All of the respondents utilized the computer daily and were adults with computer experience of at least 11 years. Tables 16, 17 and 18 show the distribution of age and their screen reader and computer experience.

Table 16 Age Distribution

\begin{tabular}{lll}
\hline \multicolumn{1}{c}{ Age } & Number of Participants & Percentage \\
\hline $40-49$ & 5 & $55.6 \%$ \\
$50-59$ & 2 & $22.2 \%$ \\
60 and over & 2 & $22.2 \%$ \\
\hline
\end{tabular}

Table 17 Screen Reader Experience

\begin{tabular}{lll}
\hline $\begin{array}{c}\text { Years of } \\
\text { Experience }\end{array}$ & Number of Participants & Percentage \\
\hline 1-5 Years & 1 & $11.1 \%$ \\
6-10 Years & 0 & $0 \%$ \\
11-20 Years & 3 & $33.3 \%$ \\
21-30 Years & 5 & $55.6 \%$ \\
\hline
\end{tabular}


Table 18 Computer Experience

\begin{tabular}{cll}
\hline $\begin{array}{c}\text { Years of } \\
\text { Experience }\end{array}$ & Number of Participants & Percentage \\
\hline $11-20$ Years & 5 & $35.7 \%$ \\
$21-30$ Years & 9 & $64.3 \%$ \\
\hline
\end{tabular}

Based on these responses, it appears that the participants have experience utilizing computers and screen readers. The specific screen reader software reported most frequently was the JAWS (five responses) screen reader, followed by the Windows Eyes (three responses) screen reader.

Applications utilized included the following: Novell GroupWise; Microsoft Outlook; Microsoft Access; Microsoft Word; Microsoft Excel, Microsoft PowerPoint; Microsoft SharePoint; Microsoft Internet Explorer; and, various blackboards. Microsoft Outlook and Microsoft Word were the top two mentioned applications, with five (5) and six (6) responses respectively. These applications were utilized by the participants in various scenarios such as: communicating through email with their virtual team members, accessing data through their WIKI database, preparing for class and participating in class discussions using asynchronous blackboards, sharing and creating co-edited documents using word processing applications; and, sharing group calendars using calendar applications. At this point, the researcher concluded that the focus group studies' participants utilized collaborative applications that included some groupware features. 


\subsubsection{Data Analysis of the Focus Group Study Recording}

The next sections will present each of the six categories identified with direct content selected from the focus group studies' recordings. The content selected will serve as an example for the analysis for each category. Content analysis was performed on the audio transcriptions and notes collected during the focus group studies, resulting in six distinct categories:

1. Participants identification of collaborative software utilized;

2. The tasks/steps necessary to complete a group project;

3. Accessibility and usability issues;

4. Group interaction techniques;

5. Accessibility documentation and support; and,

6. Accessibility design considerations.

\subsubsection{Participants identification of collaborative software utilized}

While working in groups, the participants reported that they use a screen reader to access the software application's interface. Software such as email for asynchronous communications, Chats for synchronous communication, and document sharing applications for asynchronous co-editing of documents. Additionally, a note-taking device for documenting meeting notes is utilized.

Microsoft Outlook, Microsoft Outlook Express, Novell GroupWise, Google Calendar, AOL Instant Messenger, Microsoft Office (Excel, Word, and PowerPoint), SharePoint, and Word Perfect were utilized to support group work. Outlook, Outlook 
Express, GroupWise, Instant Messenger and Google Calendar were used for their email, Chat, task tracking, and group calendars features, while Microsoft Office, SharePoint and Word Perfect were utilized for document sharing and exchanging. How these applications fit into the time-place matrix are presented in Table 19.

Table 19. Time and Place Categorization of Collaborative Technologies (Bishup et al., 2005; Ellis et al., 1991)

\begin{tabular}{|l|l|l|}
\hline & \multicolumn{1}{|c|}{$\begin{array}{c}\text { Same Time } \\
\text { (synchronous) }\end{array}$} & \multicolumn{1}{|c|}{$\begin{array}{c}\text { Different Times } \\
\text { (asynchronous) }\end{array}$} \\
\hline Same Place (local) & & \\
\hline Different Places & & - AOL IM Chat and text \\
(distributed) & messaging & - Microsoft Outlook, \\
& & $\begin{array}{l}\text { Express, Novell } \\
\text { GroupWise Email, }\end{array}$ \\
& & Google Calendar Team \\
& & scheduling and group \\
& & calendars. \\
& & - Microsoft Word, \\
& & SharePoint and Word \\
& & Perfect for document \\
& & co-editing and sharing \\
& & \\
\hline
\end{tabular}

The participants discussed adaptive technologies necessary for accessing collaborative applications. In order to interface these applications, the focus group participants utilize screen readers. The predominant screen readers utilized were the JAWS and the Window Eyes screen readers. Scripting languages are available to customize the screen reader to improve accessibility of applications the screen reader interfaces. Once it is determined that a specific application is not accessible, the screen reader's scripting language can be modified to incorporate features for accessibility. Utilizing the scripting language to improve an application's accessibility may be a possible area for further research. 
Additionally, the participants discussed the utilization of note taking devices utilized in face-to-face group meetings. The participants of the focus group studies utilize note takers, which are hardware-typing devices for documenting discussion content during group meetings. The participants mentioned using the PAC Mate and Braille Sense note takers most often.

\subsubsection{The Tasks/Steps Necessary to Complete a Group Project}

In addition to understanding the collaborative applications utilized, it was important to observe and identify the tasks or steps utilized when completing a group project. The general project workflow discussed by the participants matched prior research during the pilot focus group study which includes "identifying the purpose of the project, assigning a project leader, exchanging documents via email, followed by a combination of face-to-face meetings, conference calls and additional email exchanges."

Understanding the workflow can provide an understanding of the applications and hardware needed to support the group process. For the participants of the focus group studies, the applications and hardware mentioned prior are utilized throughout the group process. For the participants of the focus group study, there was no complete collaborative application mentioned, but a mix of many applications. A possible area for further research may be the development of a complete email, instant messaging, and document sharing integrated accessible collaborative application. Additional research is necessary to determine if this approach would be well received by persons who are blind. 


\subsubsection{Accessibility and usability issues experienced}

A recurring theme in the area of accessibility that was mentioned by the focus group participants was the inability of the screen reader to keep pace with the cursor position while reading messages in Microsoft Outlook. A participant commented, "When I arrow down through the messages, the arrow is moving down the messages, but the JAWS Screen Reader has stopped talking." One area of accessibility is the inability to access and use the track changes feature supported by Microsoft Word. Track changes corresponds with the generate task type of the Task Circumplex (Table 2), by generating new content into the document. The track changes are garbled with so many inserts and deletes that it is difficult to comprehend the changes to a document. A participant commented, "My track changes goes from the next comment to the next comment. It is hard to keep up with all of the information presented, and to determine the original and the change. I can turn on Window Eyes, to hear the changes, but I hardly ever turn that feature on, because it is too much to keep track of. Deleted two words and added a couple of words - it becomes impossible! Frequently, I just accept all of the changes. There is too much clutter with track changes."

A new approach to track changes may be another area for future research. Possibly have the original paragraph, followed by the changed paragraph in their entirety, with the ability to switch back-and-forth between views. Somehow, a complete representation of the changes may be easier to understand. Considering that the focus group participants simply accept the changes and then review. Presenting the changes in their entirety before they accept the changes may be an improved alternative approach. 
The inability to follow a conversation in a Chat session was a concern for the participants. Chat corresponds with the generate task type of the Task Circumplex (Table 2) (McGrath, 1984). A participant commented, “In a Chat Session figuring out who said what an issue is. You are no longer synchronous, when you have to look around the screen to figure out who said what. You are going to get behind the Chat pretty fast. Even if you have access, Chat is too difficult to keep up with the conversation."

Identifying the participants of a conversation is an important feature of a Chat application. In this instance, Chat would be utilized to identify and choose between several participants for conversation. This type of task would correspond to the choose task type of the Task Circumplex (Table 2) (McGrath, 1984). Future research can be utilized to focus on the issues associated with synchronous communications and solving the issues of identifying participants and keeping pace with the conversation.

As new software upgrades are made, to existing applications, the applications lose functionality and interface changes made to prior versions. A participant commented, " $I$ try to customize my interface but you lose your changes to the interface when upgrades occur. When you get a new version of Office, the new software comes out for sighted people, then the access technology people scramble to make the software function for persons who are blind." Another participant commented, "My office is using Google Calendar, which is not accessible. I now have a new assistant, who enters my schedule into Google Calendar. That type of stuff happens all the time. Some big global change takes place, and then someone thinks how will this impact a blind person, and then you are playing catch-up." 
In the same vein as upgrades to applications are cosmetic changes of new versions of these applications. Specifically, the ribbon menus added to new versions of Microsoft applications. A participant commented, "I use to be able to go to the menu bar. The ribbons are not accessible-you have the up-and-down ribbons, and you are supposed to memorize the ribbons. It is not very usable. I cannot get to the other ribbons, like the spell checker for example. The prior design with the menus and the alt keys was a better approach for accessibility and usability."

It is important that prior to upgrades or changes to existing applications that any interface changes be tested with persons with disabilities to prevent loss of service of these applications due to accessibility and usability issues. Often, persons who are blind receive upgraded applications, which either do not include their custom changes or the applications are inaccessible and unusable. Even though this rarely happens, developer awareness of the importance of retesting/reevaluating upgraded applications for accessibility and usability is critical.

\subsubsection{Group interaction techniques}

The focus group studies' participants reported that they communicate as a group by utilizing face-to-face meetings, conference calls, email, and instant messages. The approaches to group communications parallels the approaches to group communications of sighted persons. The real difference is that persons who are blind have to use additional adaptive tools such as screen readers and note takers. The screen reader provides access to applications via synthesized speech, and the note taker provides a method for documenting notes during face-to-face and conference call meetings. 


\subsubsection{Accessibility design considerations}

Modifying the collaborative applications' interface is an approach to enhancing the accessibility and usability of an interface. The ability to modify and possibly simplify the collaborative application's interface was seen as a requirement of the focus group participants. Often, a collaborative application interface component is removed from the interface to facilitate a screen reader. When the collaborative application's interface is less cluttered, persons who are blind can focus their screen reader on more precisely presented content. A participant commented, "I remove the preview pane from the interface in Outlook.”

\subsubsection{Suggestions on accessibility documentation and support}

The field studies' participants identified that it is important to have access to a support staff that can help persons who are blind with accessibility issues encountered. A participant commented, "I have IT Support. They use remote access, to login to my computer." Another participant commented, "I contact Plum Choice to remote access my computer who can look at my interface and we try to find a way a blind person can adjust the interface."

\subsection{Conclusion}

These additional focus group studies further confirmed the findings received from the pilot focus group study and the field studies. Since these participants' backgrounds were more diverse and they worked in entirely separate groups, new information in the form of accessibility and usability issues and collaborative applications were identified. 
The collaborative applications not previously identified included Microsoft Outlook Express, and Google Calendar, which are utilized for email and group calendars, Novell GroupWise and Microsoft SharePoint were solid examples of groupware, and additional word processing applications including Microsoft SharePoint and Word Perfect. Even though the variety of applications has increased, the basic tasks they support remain the same. The tasks supported are email, text messaging, task tracking, group calendar, co-editing documents, etc. Therefore, these tasks can be seen as essential to collaboration and some form of collaborative application is required to support these tasks.

Even though all the participants are mature adults with extensive experience utilizing computer and adaptive technologies while working in groups, they do encounter accessibility and usability issues while interacting with collaborative applications.

The participants of the focus group studies reported that the JAWS screen reader presented so much text that it seemed to overwhelm the end user. As a result of being overwhelmed, the end user became frustrated. What is the threshold of presented text by the JAWS screen reader that will cause the end user to be overwhelmed? Can the JAWS screen reader be adjusted to control the amount and speed of text spoken? Parsing a list of items or entries in a list by using the up-arrow and down-arrow was reported to become out-of-sync with the audio output of the JAWS screen reader, thus causing lag time and end user confusion due to reading the wrong text. Again the result of this confusion was irritation and frustration on the part of the end user. How can a list of items or entries be parsed so that skipped text can be abbreviated or not spoken? For example, as the end user parses past an item with the down-arrow or up-arrow, the screen reader should stop 
reading the item passed-over and move to the next text item. Similarly to the issue with lag time during text parsing, the JAWS screen reader did not keep pace during Chat sessions, causing participants to fall behind on the conversation, having difficulty figuring out who said what, and identifying participants of the Chat session. As a consequence of these issues, the participants terminated their use of Chat applications altogether. Can speech recognition applications be utilized to improve response time during Chat sessions, so participants can keep up with the conversation? For example, utilizing speech recognition software, the text entered for the Chat conversation can be spoken text, which may result in a faster response time. This assumes that persons can talk a response faster than they can type a response to a Chat message. Can the JAWS screen reader control when to read new messages and at what speed? How can the participants of a Chat session identify the other participants? How can we provide proper awareness to the end users when they are responding to messages as new discussions appear in synchronous environments?

Usability of tracked changes of documents was another area of major concern of the participants of the focus group studies. Tracked changes were reported to be difficult to interpret and understand by persons who are blind, since the changes read by the screen reader were garbled. The garbled changes result in persons who are blind not being able to understand the result of the changes. Often, leading them to accept the tracked change in order to read and understand the change. How can tracked changes be presented, so they are understandable to persons who are blind?

A new accessibility and usability area of concern is with Microsoft Windows' Ribbon Menus. The participants unanimously stated that ribbon menus are not accessible 
and usable. They reported that they prefer utilizing the prior drop-down menu structure. How can the ribbon menu be designed to be accessible and usable? Should the previous drop-down menu structure be optional to the ribbon menu?

All of the findings from the additional focus group studies point to the need to improve the operation between the screen reader and collaborative and other applications. Keeping pace, identifying participants, correctly navigating ribbon menus and understanding tracked changes are critical to improving accessibility and usability of collaborative applications.

The results from this study served as a guideline on what aspects of the applications need to be focused on when designing different collaborative applications/features. For example, when designing Chat features for groups of more than two people, how to present the awareness in terms of what topic is discussed by which participant is extremely important; when designing document co-editing features, how to present changes or comments made by each co-worker clearly while not clutter the whole document. 


\section{IDENTIFYING RESEARCH OPPORTUNITIES}

\subsection{Introduction}

The process of identifying the research opportunities begins with listing all of the unanswered research questions identified during the focus group studies and the field studies. The research questions are then grouped into research concerns, to logically group the unanswered research questions. The research concerns and research questions are next associated with a common collaborative purpose identified during the focus group and field studies, such as email, Chat and document co-editing (Table 20). Once the collaborative purpose is grouped to the research concerns and unanswered research questions, we can identify the support needed for each collaborative purpose.

Table 20. Collaborative Purpose, Research Concerns and Questions

\begin{tabular}{|l|l|l|}
\hline $\begin{array}{c}\text { Collaborative } \\
\text { Purpose }\end{array}$ & \multicolumn{1}{|c|}{ Research Concern } & \multicolumn{1}{c|}{ Research Questions } \\
\hline Email, Chat & Properly reading text & $\begin{array}{l}\text { How can end users be certain they are } \\
\text { reading the selected or intended message } \\
\text { properly? } \\
\text { How can they determine the number of } \\
\text { messages or range of message to make } \\
\text { certain they have not overlooked an } \\
\text { important message? } \\
\text { How can a list of items or entries be } \\
\text { parsed so that skipped text can be } \\
\text { abbreviated or not spoken? }\end{array}$ \\
\end{tabular}




\begin{tabular}{|c|c|c|}
\hline $\begin{array}{l}\text { Collaborative } \\
\text { Purpose }\end{array}$ & Research Concern & Research Questions \\
\hline Email, Chat & $\begin{array}{l}\text { Properly identifying } \\
\text { random events }\end{array}$ & $\begin{array}{l}\text { How can random events such as } \\
\text { displaying the login screen be } \\
\text { recognized by the JAWS screen reader? } \\
\text { How can the JAWS screen reader access } \\
\text { some system-level service to determine } \\
\text { if an application has lost its connection } \\
\text { or other random failure has occurred? } \\
\text { What other technique can be utilized to } \\
\text { notify a screen reader of random events? }\end{array}$ \\
\hline Chat & $\begin{array}{l}\text { Text presented } \\
\text { overwhelming the end } \\
\text { user }\end{array}$ & $\begin{array}{l}\text { How much text can be present before } \\
\text { persons who are blind are overwhelmed? } \\
\text { What is the threshold of presented text } \\
\text { by the JAWS screen reader that will } \\
\text { cause the end user to be overwhelmed? }\end{array}$ \\
\hline Chat & $\begin{array}{l}\text { Controlling the } \\
\text { conversation content and } \\
\text { speed to improve } \\
\text { understanding and } \\
\text { response time }\end{array}$ & $\begin{array}{l}\text { What method can be employed for } \\
\text { controlling the amount of text spoken by } \\
\text { the JAWS screen reader? } \\
\text { How can the text presented during a } \\
\text { Chat session be limited or controlled, so } \\
\text { not to overwhelm the end user? } \\
\text { How can speech recognition applications } \\
\text { be utilized to improve response time } \\
\text { during Chat sessions, so participants can } \\
\text { keep up with the conversation? } \\
\text { How can the JAWS screen reader } \\
\text { control when to read new messages and } \\
\text { at what speed? }\end{array}$ \\
\hline Group calendar & $\begin{array}{l}\text { Properly navigating and } \\
\text { accessing grids with a } \\
\text { screen reader }\end{array}$ & $\begin{array}{l}\text { How can complex interfaces with } \\
\text { multiple cells/dates in a grid pattern be } \\
\text { presented so a screen reader can } \\
\text { distinguish the cell content and its } \\
\text { position in the grid? } \\
\text { Alternatively, should grid structures be } \\
\text { avoided when designing interfaces that } \\
\text { are designed to be accessible and usable? } \\
\text { What other interface structure could be } \\
\text { utilized to represent a series of } \\
\text { cells/dates? }\end{array}$ \\
\hline
\end{tabular}




\begin{tabular}{|l|l|l|}
\hline $\begin{array}{l}\text { Collaborative } \\
\text { Purpose }\end{array}$ & \multicolumn{1}{|c|}{ Research Concern } & \multicolumn{1}{c|}{ Research Questions } \\
\hline $\begin{array}{l}\text { Document co- } \\
\text { editing }\end{array}$ & $\begin{array}{l}\text { Properly presenting } \\
\text { tracked changes }\end{array}$ & $\begin{array}{l}\text { How can tracked changes in a Microsoft } \\
\text { Word document be presented to a screen } \\
\text { reader that will result in content that is } \\
\text { understandable? } \\
\text { How can tracked changes be presented, } \\
\text { so they are understandable to persons } \\
\text { who are blind? }\end{array}$ \\
\hline Chat & Identifying participants & $\begin{array}{l}\text { How can the participants of a Chat } \\
\text { session identify the other participants? } \\
\text { How can we provide proper awareness } \\
\text { to the end users when they are } \\
\text { responding to messages as new } \\
\text { discussions appear in synchronous } \\
\text { environments? }\end{array}$ \\
\hline All applications & $\begin{array}{l}\text { Properly navigating } \\
\text { ribbon menus with a } \\
\text { screen reader }\end{array}$ & $\begin{array}{l}\text { How can the ribbon menu be designed to } \\
\text { be accessible and usable? }\end{array}$ \\
\hline
\end{tabular}

If we were to propose a collaborative application that included Chat, we would want to include support for properly handling random events, reading Chat text, identifying participants, avoiding overwhelming end users with too much presented text, and enabling control over the amount of text presented. For proposing a collaborative application that included email, we would want to include support for properly handling random events and reading email message text. If the collaborative purpose involved a group meeting utilizing a calendar, we would want to include support for properly navigating and accessing days of a calendar grid. Finally, if the collaboration involved co-editing documents, we would want to include support for properly tracking document changes. The changes could include inserts, deletes, changes, adding and removing comments, etc. For all of the previously mentioned collaborative purposes properly navigating a ribbon menu may be necessary. 
Most prior research (Hampel et al., 1999; Takagi et al., 2000; Thiessen et al., 2007; Woodfine et al., 2008) focused on solving accessibility and usability issues related to Chat and email collaborations, with less focus on the accessibility and usability of the collaborative writing tasks. Therefore, there appears to be a greater opportunity for valuable research in regard to collaborative writing and the access and use of track changes.

\subsection{Collaborative Writing Research}

Some recent research (Dorigo et al., 2011; Schoeberlein \& Wang, 2009b; Buzzi et al., 2010a) focused on the accessibility and usability of electronic documents in general, and not on the access and use of collaborative writing features of these documents such as track changes and revisions. Dorigo (2011) reported that 205 respondents to the survey wanted better access to electronic documents as well as an overview of the documents' structure and content. Schoeberlein and Wang (2009b) scanned the Google Docs web interface with an automated tool to examine its accessibility and discovered that the Google Docs web interface had accessibility issues. Automated tools can identify "false positives" in regard to accessibility issues. Therefore, it is important to manually verify the automated tool results against actual found issues. The automated tool should only be a starting point for examining an application for accessibility. Automated tools can only identify certain accessibility issues and will not identify usability issues or all accessibility issues. Buzzi (2010) conducted a usability study examining Google Docs with the use of a screen reader. Buzzi (2010) identified orientation to the interface as not efficient or satisfactory, accessing basic functions as not perceivable and operable, 
control elements (buttons, links, etc.) were unclear and not understandable, and working with the documents is nearly impossible via the keyboard.

Even though these studies are not focused specifically on collaborative writing, they identify the needs of persons who are blind when interacting with electronic documents, and present general accessibility and usability issues of collaborative writing environments. The National Federation of the Blind filed a civil rights lawsuit with the Department of Justice in regard to the adoption of Google Apps by Universities in New York and Oregon ("National Federation of the Blind Lawsuit," 2013). The claim was that Google Apps was not compatible with screen readers and prevented access to persons who are blind ("National Federation of the Blind Lawsuit," 2013).

Three approaches to developing a collaborative writing interface prototype as part of this dissertation were previously discussed in section, "3.8 Potential Approaches for Developing Accessible Prototypes." One of these approaches was WAI-ARIA ("W3C," 2006). Buzzi (2008) applied WAI-ARIA Metadata to improve the Login, document list, and text editing formatting features of Wikipedia. In later research, Buzzi applied WAIARIA Metadata to improve the formatting toolbar and text editing formatting features of Google Docs. Even though the application of WAI-ARIA Metadata is not specific to collaborative writing features such as track changes and revision histories, Buzzi did illustrate the viability of WAI-ARIA Metadata for improving accessibility for Web-based collaborative applications.

Specific research in regard to collaborative writing included Buzzi's (2011) work with Google Docs. After examining the Google Docs interface, Buzzi noted that the Google Docs interface had access and use issues when publishing a web page, uploading 
files, announcing the revision differences by the JAWS Screen Reader, and distinguishing the differences between a comment and text by the JAWS Screen Reader. This supports the claim by the NFB in their civil rights lawsuit in regard to access and use of Google Apps ("National Federation of the Blind Lawsuit," 2013). Buzzi suggested improving the collaborative writing environment; the application should operate via the keyboard, provide awareness of other collaborators, provide an understanding of where the other collaborators were editing the document, and provide alerts for messages and feedback. Even though Buzzi identified access and use issues found when interacting with the Google Docs interface, this research was limited to a total of four participants, with only one participant who was blind. More participants who are blind are needed to improve the validity of the findings from Buzzi. The other three participants shut off the monitor, to simulate blindness. Again, persons who are blind must participate in order for valid results to be justified. Therefore, additional studies are necessary with additional participants in order to validate and expand on Buzzi's findings.

Additional collaborative writing research should be conducted which builds on this prior research and provides a comparison of access and use between persons who are blind and persons who are not blind, examines the effects of co-editing the same text at the same time, and examines collaborative writing features such as adding, changing and deleting text and comments. 


\subsection{Manual Examination of Google Docs and Microsoft Word}

In order to gain a better understand of the potential issues related to collaborative writing, the researcher of this dissertation examined Microsoft Word's and Google Docs' collaborative writing features. Microsoft Word was chosen to represent asynchronous desktop collaborative writing applications and Google Docs was chosen to represent a web-based application that supports both asynchronous and synchronous collaborative writing tasks. Microsoft Word's approach to collaborative writing is to utilize a track changes feature that marks document changes. Whereas, Google Docs' approach to collaborative writing is to show the change instantly on the screen as the collaborators cowrite a document at the same time.

Examining Microsoft Word and Google Docs began by following the task lists (Appendix I - Microsoft Word, Appendix K - Google Docs) developed by the researcher of this dissertation. During these examinations, the researcher utilized the JAWS Screen Reader and the keyboard for interaction in order to simulate the interactions of persons who are blind.

While examining Microsoft Word, most tasks (Appendix I) were completed with the exceptions of searching, accepting and rejecting changes, since these features were not keyboard accessible. One alternative that would provide direct keyboard access to these features would be to use hot-keys. Hot-keys could be programmed in order to allow direct keyboard access, and provide access for persons who are dependent on keyboard access.

While examining Google Docs, most tasks (Appendix K) were completed with the exception of searching through the changes. Even though access to the revisions' 
history was facilitated, scrolling through and editing a selected document version was not possible. Access to the document was prevented due to the lack of keyboard focus moving from the revisions' history list to the selected document version. Google Docs allows for the acceptance or rejection of a specific document version, which may contain one or more modifications. However, it does not allow for the acceptance or rejection of one modification among others in one version. Therefore, if you chose to restore a prior version of the document in the revisions' history list, you could accidentally remove some changes you did not intend to remove.

Google Docs was also examined to determine how it functions during synchronous use of a shared document. Two collaborators were able to type content simultaneously and see the content as both collaborators typed. As the first participant was typing, the content typed by the second participant appeared in the first participant's line-of-sight, causing the first participant to pause and read the other content. Thus, the simultaneous editing of the document was distracting to the first participant. 


\subsection{Conclusion}

In this Chapter, the research opportunities were identified from the focus group and field studies. Based on the research opportunities identified, collaborative writing was identified as the research topic of this dissertation. As a starting point, recent literature was reviewed and the collaborative writing features of Microsoft Word and Google Docs were examined. These examinations provided a starting point for the possible accessibility and usability issues persons who are blind may experience while collaborating.

The next Chapter's focus is on the research design and methodology for determining the accessibility and usability issues of collaborative writing technologies, and identifying how a collaborative writing interface should be designed in order to be accessible and usable for persons who are blind. 


\section{COLLABORATIVE WRITING RESEARCH DESIGN AND METHODOLOGY}

\subsection{Introduction}

The previously reported focus group studies (Schoeberlein \& Wang, 2011) presented evidence that collaborative technologies (e.g. Chat, group calendars, email, and collaborative writing applications) are not accessible and usable. Accessibility and usability issues occurred during interactions between the JAWS Screen Reader and the application interface. During Chat sessions, the text read by the screen reader would overwhelm the end user until they could no longer participate. While interacting with group calendars, it was difficult to distinguish a particular date in the calendar. Searching and reviewing revisions and comments within a document, it became difficult to distinguish the original text from the inserted text and comments, leading to confusion and frustration. These accessibility and usability issues lead to the discontinued use of the application, which prevents persons who are blind from fully participating in collaborative work environments.

An iterative approach to designing an accessible collaborative writing interface was undertaken by gradually improving the interface. 
The following research questions were examined:

- RQ1: What accessibility and usability issues do collaborative writing technologies, such as Microsoft Word and Google Docs, present for persons who are blind?; And,

- RQ2: How should a collaborative writing interface is designed in order to be accessible and usable for persons who are blind?

This research included a baseline study, the design of a prototype, a pilot study, and enhancements to the prototype and a final usability study. The baseline usability study was utilized to identify the accessibility and usability issues associated with track change and commenting during collaborative writing. As part of the baseline usability study, Microsoft Word and Google Docs were evaluated. At the completion of the baseline usability study, a solution prototype was designed which solves the accessibility and usability issues identified. A pilot usability study was used to gather feedback to tweak the prototype, before the final usability study was undertaken. The data collected from all rounds of usability study were analyzed.

The purpose of this chapter is to present an overview of the five phases and the research methods utilized to understand the collaborative access and use needs of the participants and to propose an accessible and usable collaborative writing interface. The details and results of each of the five phases and the research methods are presented in Chapter 10. 


\subsection{Research Method}

\subsubsection{Phase One - Baseline Usability Studies}

The first phase was a baseline usability study examined Google Docs' and Microsoft Word's collaborative writing features. The purpose of the baseline usability study was to identify the baseline performance measurements, the key obstacles, and the preferences of the participants. The baseline performance measurements were utilized to compare against the performance measurements of the prototype, to determine if there was any performance improvement. Any key obstacles identified were resolved in order to continue with the next phases of this research. Preferences of the participants were utilized as requirements for the collaborative writing prototype. 


\subsubsection{Procedure}

The usability study procedure followed these steps:

1. Recruit the participants;

2. Select the test environment;

3. Present informed consent for signature (Appendix G);

4. Collect demographical and experience data via a pre-study survey (Appendix H);

5. Conduct the usability study session, using the task lists (Appendix I - MS Word, Appendix K - Google Docs), with “Amendments of the United States Constitution" (Appendix J);

6. Record task performance (including the successfulness of the task) directly next to each task on the task list;

7. Collect audio/video recordings and researcher notes;

8. Collect user satisfaction survey(s) (Appendix C);

9. Debrief participants;

10. Perform quantitative and qualitative data analysis; and, 11. Report findings. 


\subsubsection{Participants and Study Location}

The Researcher recruited the participants for the baseline usability studies from the Members of the National Federation of the Blind (NFB) and the students from local universities. Five (5) participants meeting the criteria listed were recruited:

- A person who is blind with no residual vision;

- A person who utilizes Microsoft Word or Google Docs two or more times per week;

- A person who utilizes the JAWS Screen Reader two or more times per week; and, - A person who utilizes their Computer two or more times per week. Seven (7) visually able participants were recruited. The criteria for selecting participants who are not blind were the same as the blind group except for the vision requirement and the use of the JAWS Screen Reader. The baseline usability studies were conducted at the National Federation of the Blind (NFB), Towson University and various other locations dependent on the participants' needs. 


\subsubsection{Task Lists}

The tasks of the baseline usability study included typical tasks associated with collaborative writing such as:

- Review changes (Parse the changes in the document);

- Add/insert changes / comments;

- Delete changes / comments;

- Accept changes / comments; and,

- Reject changes / comments.

\subsubsection{Study Sessions}

Prior to the study sessions, each participant completed a pre-study survey questionnaire that indicated the participants' prior experience with Microsoft Word and Google Docs. This study included seven visually able participants and five participants who are blind. The pre-study survey questionnaire was used to verify the participants' qualifications for this study. Since the participants who were blind did not utilize Google Docs on a regular basis, we did not ask them to perform interactions with Google Docs. The participants who were not blind examined both Microsoft Word and Google Docs. The usability study sessions were conducted using the original interfaces of Microsoft Word and Google Docs. A task list guideline (Appendix I - MS Word, Appendix K Google Docs) details the tasks completed during the usability studies' sessions. The participants were encouraged to focus on the timed tasks and to "talk aloud" only if they became frustrated or need assistance. Five minutes was allotted for each task, before the 
participants were asked to move on to the next task. A maximum time limit of 90 minutes was allotted for each participant to complete the usability study, the pre-survey and postsurvey. The participants' interactions with the interface were audio recorded. Both audio transcripts and detailed researchers' notes were collected during the baseline usability studies. The transcripts and notes were coded based on the themes that emerge from the studies' observed events. Task completion and the time spent were collected for each task in the study task list. User satisfaction was collected via a post study questionnaire (Appendix C) at the conclusion of the baseline usability studies.

9.2.2 Phase Two - Development of an accessible and usable collaborative writing prototype

The purpose of the second phase was to develop an accessible and usable collaborative writing interface prototype, based on the preferences of persons who are blind and the performance measurements of the baseline usability study.

The development environment utilized was Microsoft Visual Studio (2010, 2013), and the interface prototype was developed in Microsoft Visual Basic (2010, 2013). The interface was designed as a Microsoft Word Add-In (Word Add-Ins), which included a context-sensitive interface in the form of a Microsoft Word Command Bar ("MSDN Microsoft Word Command Bar," 2013). The focus of the prototype's interface was on presenting the revisions and comments in a format that was accessible and usable for persons who are blind. 
Based on the findings from the baseline usability study, interface design requirements were identified. The development life cycle followed these steps:

1. Identified the participants' requirements for the interface design;

2. Developed and tested the collaborative writing prototype; and,

3. Deployed the collaborative writing prototype to the usability studies' environment.

\subsubsection{Phase Three - Pilot Usability Study}

The purpose of the pilot usability study phase was to validate the initial prototype interface, in order to determine if the prototype was accessible/usable and compatible with the JAWS Screen Reader. No key obstacles were observed and the initial prototype was determined to be compatible with Microsoft Word and the JAWS Screen Reader. Preferences of the participant were utilized as requirements for improving the collaborative writing prototype. This phase was important in order to improve the prototype's interface before the additional two rounds of usability study.

\subsubsection{Procedure and Tasks}

The procedure followed the same steps as the Baseline Usability Study in section 9.2.1.1. The tasks of the usability study followed the collaborative writing tasks associated with searching, and accepting and rejecting changed text throughout the document. 


\subsubsection{Participant}

The participant, who was blind, was an experienced user of Microsoft Word and the JAWS Screen Reader. The choice of utilizing one experienced participant was to validate the basic operations of the prototype and not intended as a full-blown usability study. The pilot usability study was intended to prove the soundness of the prototype design and the usability study procedure and measurements.

\subsubsection{Usability Study Session}

The pilot usability study session was conducted using the developed collaborative writing prototype. Audio was recorded to generate a transcript for analysis, and detailed notes of the session were collected. The same qualitative and quantitative measurements as the baseline usability study were also collected.

\subsubsection{Phase Four - Enhancement of the collaborative writing prototype}

Based on the suggestions collected from the pilot usability study, the prototype was improved by clarifying some of the wording of the Microsoft Word Command Bar options and the Message Boxes. The same development environment utilized in phase two to develop the collaborative writing prototype was utilized to enhance the collaborative writing prototype. 


\subsubsection{Phase Five - Final Usability Study}

The fifth and final phase was a final usability study examining the improved collaborative writing prototype developed in phase four. The procedure and tasks followed the same steps as the Pilot Usability Study. Eleven participants who are blind were recruited for the additional rounds of usability study utilizing the same criteria utilized for the Baseline Usability Study. The final usability study sessions were conducted using the collaborative writing prototype enhanced during phase four of this research.

\subsection{Data Collection Methods}

Data collection from all usability study consisted of the five sources including questionnaire responses, task completion and times spent, video recordings; transcript of the audio recording from the usability studies; and, the Researcher's notes taken of observed events during the usability studies.

The questionnaires provide background, demographical and satisfaction data. The task completion and time spent provide quantitative data for each task. The video/audio recording and notes provide qualitative data to gather user behavior about collaborative writing of documents for persons who are blind.

Both pretest and posttest questionnaires were provided online, using Survey Gizmo (Survey Gizmo). Each participant was asked to complete the pre-study and poststudy questionnaire. The pretest questionnaire included questions for demographical and experience data (Appendix H). The posttest questionnaire included questions for user satisfaction (Appendix C/D). 
9.4 Qualitative Data Analysis Method - The Grounded Theory

The Grounded Theory (2009) was utilized to help interpret the audio data recorded during the usability study sessions. Agreement among coders for each category was calculated using SSPS (2010) and Cohen's Kappa (2010). An agreement of 70\% among coders is considered sufficient (Cohen's Kappa, 2010).

\subsection{Quantitative Data Analysis Method}

For all rounds of usability study, the set of quantitative data was used to compare the performance of the interface design. The task performance and time performance were compared between usability studies, to determine if an improvement has occurred for the prototype design. For the task performance, the mean and standard deviation were calculated for the number of correctly completed tasks. For the time performance, the mean and standard deviation were calculated for how long each task took to correctly complete. Additionally, the percentage of task completion was calculated, to determine the tasks that required additional examination. The findings cannot be generalized, but the comparisons were used to determine whether there was any improvement with the prototype design. 


\section{APPLICATION DESIGN AND USABILITY EVALUATIONS}

\subsection{Introduction}

This chapter begins with a baseline usability study, which is utilized to identify the collaborative writing needs of the participants who are blind. Following the baseline usability study, a design of an accessible and usable interface for track change and comments was implemented as "CollaborateWithWord." After the development of the prototype, a pilot usability study session was conducted to validate the prototype's compatibility with the JAWS Screen Reader and to improve the interface. The final usability study was conducted with additional participants to further examine the prototype. The final usability study consisted of one round of usability study, followed by additional enhancements to the prototype and a second round of usability study.

\subsection{Baseline Usability Study}

The baseline usability study consisted of two distinct sets of participants during several distinct usability study sessions. Multiple usability study sessions were carried out between March and August 2012, with participants who were visual users and blind users. The purpose of the baseline usability study was to identify accessibility and usability issues while engaged in collaborative writing.

\subsubsection{Participants}

The participants were recruited from the Members of the NFB and Students from local Colleges and Universities. Fifteen participants carried out the study sessions, in which seven participants were visually able and eight participants were blind. The 
specific selection criteria for participants who are blind can be found in section "9.2.1.2 Participants and Study Location."

\subsubsection{Environment Configurations}

The visually able and blind participants carried out the tasks on a Hewlett Packard Elite Book running Windows Vista, with access to Microsoft Word 2010, Google Docs and Internet Explorer 8 installed.

\subsubsection{Procedure}

The baseline usability study sessions began with a brief introduction of the purpose of the study, followed by the signing of an informed consent form (Appendix G). Each participant completed a pre-study questionnaire to collect background information about the participants (Appendix H). Following the completion of the pre-study questionnaire, each participant performed the tasks from the task lists (Appendix I - MS Word, Appendix K - Google Docs) in separate sessions. Each participant examined and edited the document (Appendix J), "Amendments of the United States Constitution." During the usability study session, the researcher recorded task performance (complete or incomplete), task completion time (time to complete) directly next to each task on the task list. Audio, video and researcher notes were collected. Following the usability study, a brief post-study questionnaire was collected on the task experience and opinions of likes and dislikes, and how to improve the interfaces of the system they interacted with during the study session. Each study session ran for about 90 minutes per participant. 


\subsubsection{Tasks}

The collaborative writing tasks (Table 21) included tasks associated with opening a document, initiating the track change feature, adding, deleting and changing text of a document, adding a comment, and searching, accepting and rejecting changed text throughout the document.

Since Google Docs keeps track of document changes based on time increments and several changes are accepted at the same time, there is no practical way to accept or reject individual changes. Therefore, accepting and rejecting changes was not conducted with Google Docs. Additionally, since Google Docs is a synchronous collaborative writing application, a collaborator was invited to the writing session.

Table 21. Usability Study - Tasks Included by Application

\begin{tabular}{|l|l|l|}
\hline \multicolumn{1}{|c|}{ Task } & \multicolumn{1}{|c|}{ Microsoft Word } & \multicolumn{1}{|c|}{ Google Docs } \\
\hline 1. Open the Document & 1. Included & 1. Included \\
2. Start "Track Changes" & 2. Included & 2. Not Included \\
3. Invite a collaborator & 3. Not Included & 3. Included \\
4. Add a change & 4. Included & 4. Included \\
5. Delete text & 5. Included & 5. Included \\
6. Change text & 6. Included & 6. Included \\
7. Add a comment & 7. Included & 7. Included \\
8. Search for all changes & 8. Included & 8. Included \\
9. Accept a change & 9. Included & 9. Not Included* \\
10. Reject a change & 10. Included & 10. Not Included* \\
11. Accept/reject changes & 11. Included & 11. Not Included* \\
\hline
\end{tabular}

* Accept/reject individual changes are not provided by Google Docs; therefore, these items are not included. 


\subsubsection{Data Collection}

Both qualitative and quantitative data were collected in each study session. The data sources from the baseline usability study included the responses to pre-study and post-study questionnaires, the task list completion times, the researcher's notes, and the audio and video recordings. Table 22 presents the data variable, the data source, the type and use of the data collected for analysis. The researcher and an assistant coded the qualitative data to identify categories for validation. The inter-coder reliability was calculated using Cohen's Kappa (2010). A Cohen's Kappa (2010) 70\% agreement among the coders is considered a reliable category interpretation of the data. The coders agreed $83.9 \%$ on 45 cases, providing evidence that the data interpretation was reliable. 
Table 22. Data Variables, Sources, Types, and Measurements

\begin{tabular}{|c|c|c|c|}
\hline Variable & Data Source & $\begin{array}{c}\text { Type of } \\
\text { Data } \\
\text { Collected }\end{array}$ & Measurement \\
\hline Demographic & $\begin{array}{l}\text { Pre-study and } \\
\text { post-study } \\
\text { questionnaires }\end{array}$ & Quantitative & $\begin{array}{l}\text { Averages and standard } \\
\text { deviations for ranges of } \\
\text { demographical questions }\end{array}$ \\
\hline \multirow[t]{2}{*}{ Perception/Satisfaction } & $\begin{array}{l}\text { Pre-study and } \\
\text { post-study } \\
\text { questionnaires/ }\end{array}$ & Quantitative & $\begin{array}{l}\text { Percentages of the } \\
\text { participants who agreed or } \\
\text { disagreed with a specific } \\
\text { statement }\end{array}$ \\
\hline & $\begin{array}{l}\text { Researcher's } \\
\text { Notes and } \\
\text { video } \\
\text { Recordings }\end{array}$ & Qualitative & 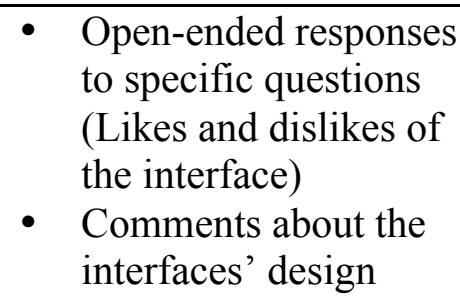 \\
\hline \multirow[t]{2}{*}{ Performance } & $\begin{array}{l}\text { Task list and } \\
\text { video } \\
\text { recordings }\end{array}$ & Quantitative & 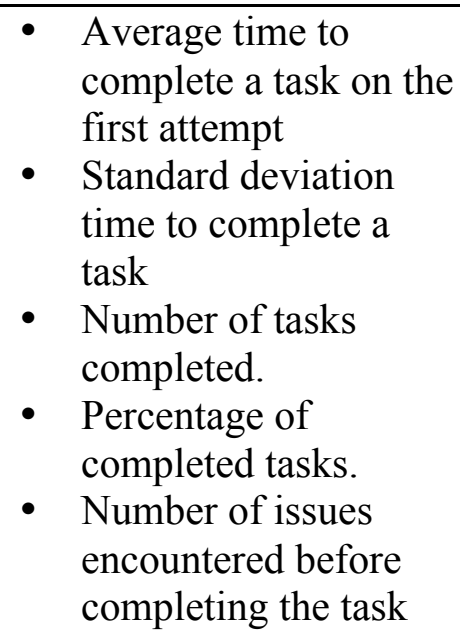 \\
\hline & $\begin{array}{l}\text { Researcher's } \\
\text { Notes and } \\
\text { Audio/Video } \\
\text { Recordings }\end{array}$ & Qualitative & $\begin{array}{l}\text { Data in regard to observed } \\
\text { events such as searching } \\
\text { techniques, accessibility } \\
\text { and usability issues }\end{array}$ \\
\hline
\end{tabular}




\subsubsection{Results}

The purpose of data analysis is to examine the data collected and identify central tendencies and the distribution in order to describe facts, identify patterns, and develop explanations ("Data Analysis," 2013).

\subsubsection{Demographics}

Demographical and prior experience data was collected from the responses collected from the pre-study questionnaire. Table 23 presents the data collected from visually able participants and participants who are blind. The visually able participants do not require a screen reader to access computer applications, so no data regarding screen reader use is reported. All of the participants who are blind reported that they did not utilize Google Docs due to access issues. Therefore, no data is reported for the participants who are blind in regard to Google Docs. 
Table 23. Demographical Data - Visually Able Participants and Participants who are Blind

\begin{tabular}{|c|c|c|}
\hline Demographical Data & $\begin{array}{c}\text { Visually able } \\
\text { participants } \\
\% \text { (\#) }\end{array}$ & $\begin{array}{c}\text { Participants } \\
\text { Who Are } \\
\text { Blind } \\
\%(\#)\end{array}$ \\
\hline \multicolumn{3}{|c|}{ Gender } \\
\hline Male & $86 \%(6)$ & $50 \%(4)$ \\
\hline Female & $14 \%(1)$ & $50 \%(4)$ \\
\hline \multicolumn{3}{|c|}{ Age Range } \\
\hline Age 19 to 29 & $58 \%(4)$ & $37.5 \%(3)$ \\
\hline Age 30 to 39 & $14 \%(1)$ & $25 \%(2)$ \\
\hline Age 40 to 49 & $14 \%(1)$ & $25 \%(2)$ \\
\hline Age 50 to 59 & $14 \%(1)$ & $12.5 \%(1)$ \\
\hline \multicolumn{3}{|c|}{ Computer Experience Level } \\
\hline Less than 1 year of computer experience & $0 \%(0)$ & $12.5(1)$ \\
\hline 6 to 10 years of computer experience & $29 \%(2)$ & $25 \%(2)$ \\
\hline 11 to 20 years of computer experience & $14 \%(1)$ & $12.5 \%(1)$ \\
\hline 21 to 30 years of computer experience & $57 \%(4)$ & $50 \%(4)$ \\
\hline \multicolumn{3}{|c|}{ Computer Use Frequency } \\
\hline Daily & $100 \%(7)$ & $100 \%(8)$ \\
\hline \multicolumn{3}{|l|}{ Primary Purpose } \\
\hline Computer for work & $57 \%(4)$ & $62.5 \%(5)$ \\
\hline Computer for school / college & $29 \%(2)$ & $37.5 \%(3)$ \\
\hline Computer for entertainment & $14 \%(1)$ & $0 \%(0)$ \\
\hline \multicolumn{3}{|c|}{ JAWS Experience Level } \\
\hline 1 to 5 years & NA & $37.5(3)$ \\
\hline 6 to 10 years & NA & $12.5 \%(1)$ \\
\hline 21 to 30 years & NA & $50 \%(4)$ \\
\hline
\end{tabular}




\begin{tabular}{|c|c|c|}
\hline Demographical Data & $\begin{array}{c}\text { Visually able } \\
\text { participants } \\
\%(\#)\end{array}$ & $\begin{array}{c}\text { Participants } \\
\text { Who Are } \\
\text { Blind } \\
\% \text { \% (\#) }\end{array}$ \\
\hline \multicolumn{3}{|c|}{ Microsoft Word Experience Level } \\
\hline Less than 1 year & $14.3 \%(1)$ & $12.5 \%(1)$ \\
\hline 1 to 5 years & $0 \%(0)$ & $37.5 \%(3)$ \\
\hline 6 to 10 years & $57.1 \%(4)$ & $0 \%(0)$ \\
\hline 11 to 20 years & $14.3 \%(1)$ & $50 \%(4)$ \\
\hline 21 to 30 years & $14.3 \%(1)$ & $0 \%(0)$ \\
\hline \multicolumn{3}{|c|}{ Microsoft Word Use Frequency } \\
\hline Daily & $14 \%(1)$ & $50 \%(4)$ \\
\hline Several times a week & $87 \%(6)$ & $50 \%(4)$ \\
\hline \multicolumn{3}{|c|}{ Google Docs Experience Level } \\
\hline No response & $0 \%(0)$ & $37.5 \%(3)$ \\
\hline 1 year or less & $57.1 \%(4)$ & $62.5 \%(5)^{*}$ \\
\hline 1 to 5 years & $42.9 \%(3)$ & $0 \%(0)$ \\
\hline \multicolumn{3}{|c|}{ Google Docs Use Frequency } \\
\hline No response & $0 \%(0)$ & $100 \%(8)$ \\
\hline Daily & $14 \%(1)$ & $0 \%(0)$ \\
\hline Several times a week & $87 \%(6)$ & $0 \%(0)$ \\
\hline
\end{tabular}

* Participants who are blind attempted to utilize Google Docs at most one time and due to accessibility issues discontinued use. Therefore, the Participants who are blind only examined Microsoft Word.

All $(100 \%)$ of the visually able participants and the participants who are blind met the experience and use criteria for the baseline usability study using Microsoft Word. The majority of visually able participants were male (84\%) participants. The participants who are blind were evenly distributed between male participants $(50 \%)$ and female participants $(50 \%)$. The visually able participants and the participants who are blind had similar distributions in regard to their age range, computer experience, computer use frequency, and primary use of the computer. Even though the participants who are blind 
reported Google Docs experience of "1 year or less," they actually attempted to utilize Google Docs at most one time and due to accessibility issues discontinued use. The participants who are blind were able to open Google Docs, and interact with the Web Browser Menu. However, they could not access the menu options or the revisions list within the Google Docs interface. The Google Docs interface is contained within the Web Browser interface. Therefore, Google Docs was not a focus for the participants who are blind. They examined Microsoft Word.

\subsubsection{Task Performance}

During the baseline usability study, the begin time and end time was recorded for each task in the task list. The time range was quantified as an average task completion time and a standard deviation. The central tendency and deviation can be utilized to determine if one group of participants was out-of-range with another group. For example, do participants who are blind complete each task within the similar range (average +- the standard deviation) as visually able participants? Table 24 presents the average rate of task completion for visually able participants, and Table 25 presents the task completion success rate, mean and standard deviation for the participants who are blind. The task completion time includes time for "talk-out-loud," which may influence the "real" completion time. The completion percentages present actual successfully completed tasks. 
Table 24. Baseline Usability Study - Task Performance for Visaually Able Participants

\begin{tabular}{|c|c|c|c|c|}
\hline \multirow[t]{2}{*}{ Task } & \multicolumn{2}{|c|}{ Microsoft Word } & \multicolumn{2}{|c|}{ Google Docs } \\
\hline & $\begin{array}{c}\text { Task } \\
\text { Completion } \\
\text { Success Rate }\end{array}$ & $\begin{array}{l}\text { Time Spent } \\
\text { Mean/Std. } \\
\text { Deviation } \\
\text { (Seconds) }\end{array}$ & $\begin{array}{c}\text { Task } \\
\text { Completion } \\
\text { Success Rate }\end{array}$ & $\begin{array}{l}\text { Time Spent } \\
\text { Mean/Std. } \\
\text { Deviation } \\
\text { (Seconds) }\end{array}$ \\
\hline $\begin{array}{l}\text { Login and Invite a } \\
\text { collaborator }\end{array}$ & NA & $\mathrm{NA}$ & $100 \%$ & $42.87 / 34.01$ \\
\hline $\begin{array}{l}\text { Open the } \\
\text { Document and } \\
\text { Start "Track } \\
\text { Changes" }\end{array}$ & $100 \%$ & $42.85 / 16.03$ & $100 \%$ & $55.71 / 43.91$ \\
\hline Add a change & $100 \%$ & $120 / 34.64$ & $100 \%$ & $64.28 / 26.99$ \\
\hline Delete text & $100 \%$ & $42.85 / 16.03$ & $100 \%$ & $38.57 / 14.63$ \\
\hline Change text & $100 \%$ & $38.57 / 14.63$ & $100 \%$ & $30 / 0$ \\
\hline Add a comment & $100 \%$ & $42.85 / 16.03$ & $100 \%$ & $30 / 0$ \\
\hline $\begin{array}{l}\text { Search for all } \\
\text { changes }\end{array}$ & $100 \%$ & $51.42 / 14.63$ & $100 \%$ & $60.85 / 63.91$ \\
\hline Accept a change & $100 \%$ & $42.85 / 16.03$ & NA & NA \\
\hline Reject a change & $100 \%$ & $60 / 30$ & NA & NA \\
\hline $\begin{array}{l}\text { Accept/reject } \\
\text { remaining changes }\end{array}$ & $100 \%$ & $47.14 / 16.03$ & NA & NA \\
\hline
\end{tabular}

All visually able participants completed all tasks evaluated for Microsoft Word and Google Docs (Table 24). The visually able participants took more time when adding a change, deleting a change, changing the text and adding a comment, while interacting with Microsoft Word versus Google Docs. Adding a change took the longest time to complete and took almost twice as long when utilizing Microsoft Word versus Google Docs. Reviewing the video, it appears that the participants used the Microsoft Word "Search" feature, when trying to find the insertion points for the text. The "search" feature reset the searches to the beginning of the document, causing a longer seek time 
before finding the text insertion point. However, when inserting text into a Google Docs document, the participants manually scrolled to the insertion points; thus, improving task completion time for adding a change.

Table 25. Baseline Usability Study - Task Performance for Participants Who Are Blind

\begin{tabular}{|c|c|c|}
\hline \multirow[t]{2}{*}{ Task } & \multicolumn{2}{|c|}{ Microsoft Word } \\
\hline & $\begin{array}{c}\text { Task Completion Success } \\
\text { Rate }\end{array}$ & $\begin{array}{l}\text { Time Spent Mean/Std. } \\
\text { Deviation (Seconds) }\end{array}$ \\
\hline $\begin{array}{l}\text { Login and Invite a } \\
\text { collaborator }\end{array}$ & NA & NA \\
\hline $\begin{array}{l}\text { Open the Document and } \\
\text { Start "Track Changes" }\end{array}$ & $100 \%$ & $30.63 / 13.74$ \\
\hline Add a change & $100 \%$ & $252 / 75.52$ \\
\hline Delete text & $100 \%$ & $78.12 / 26.04$ \\
\hline Change text & $100 \%$ & $70.62 / 29.69$ \\
\hline Add a comment & $100 \%$ & $202.5 / 73.77$ \\
\hline Search for all changes & $62.5 \%$ & $155 / 67.82$ \\
\hline Accept a change & $62.5 \%$ & $73 / 14.83$ \\
\hline Reject a change & $62.5 \%$ & $89 / 63.38$ \\
\hline $\begin{array}{l}\text { Accept/reject remaining } \\
\text { changes }\end{array}$ & $62.5 \%$ & $249 / 120.38$ \\
\hline
\end{tabular}

The comparison between visually able participants' and participants' who are blind results for the Microsoft Word tasks (Tables 24 and 25), showed unlike visually able participants, not all of the participants who are blind were able to complete all of the tasks. Less than two-thirds of the participants who are blind completed the searching and accepting/rejecting changes tasks (Table 25). Additionally, they took longer on average to complete all of the tasks, except opening the document and starting track changes. When compared to visually able participants, the participants who are blind took almost twice or more time to complete most tasks (Figure 18). 


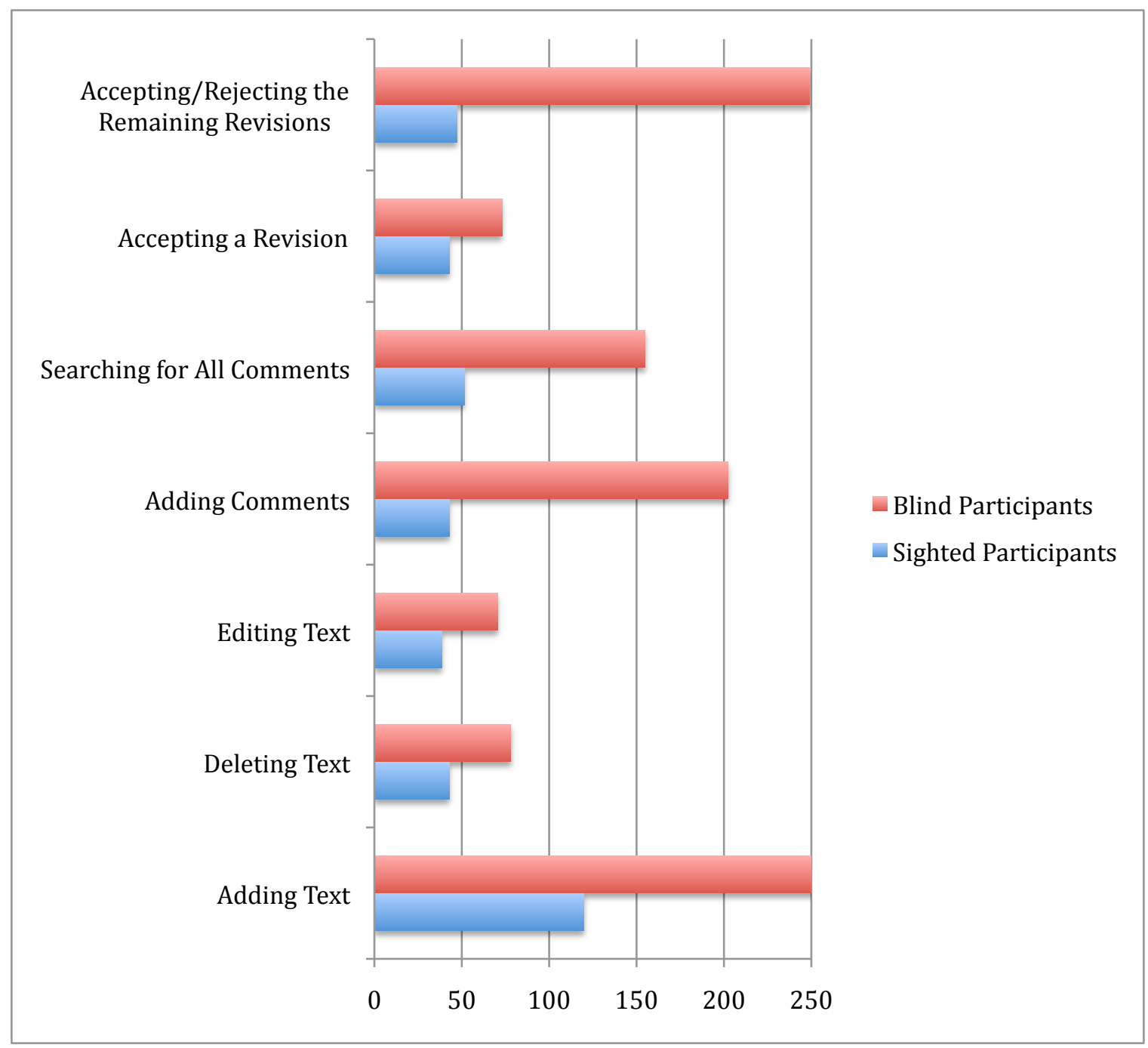

Figure 18 Task Completion Times in Seconds

For the participants who are blind, the lower performance can be attributed to the time required to find specific menu options, and to locate the correct positions for adding or changing text. Microsoft Word menu options for track change functions (previous/next change, accept/reject change, previous/next comment) are directly accessible with the keyboard, but require complex key-press combinations and require memorization to become efficient. Alternatively, to using the key-press combinations to access the track change functions, all participants who are blind searched the entire menu structure manually with the tab key press resulting in higher search/seek times. 
The JAWS Screen Reader speed setting ranges from 40 (slow) to 150 (fast). For this study the JAWS Screen Reader was set to "Eloquence" at the default speed setting of 57, which is a normal speech pace. The default speed setting was used for all participants of this study. Adjustments were not made to increase or decrease the voice speed. Search/seek times may improve by making adjustments to the JAWS Screen Reader speed.

\subsubsection{Analysis of the audio/video recordings}

For the analysis of the audio/video recordings, transcripts were developed and coded from the baseline usability study. Two independent coders examined and coded the transcripts. Forty-five cases that belong to five categories were identified. The coders agreed on $83.9 \%$ of the cases providing acceptable agreement and reliability.

Five categories were identified from the annotated list collected while reviewing the Microsoft Word audio/video transcripts:

1. Searching techniques;

2. Selecting techniques;

3. Editing techniques;

4. Reading techniques; and,

5. Accessibility and usability issues.

Table 26 presents the categories identified from transcripts of the visually able participants and the participants who are blind from the usability study audio/video recordings collected during the examination of Microsoft Word. 
Table 26. Category and Annotated List Element

\begin{tabular}{|c|c|c|}
\hline Category & $\begin{array}{l}\text { Visually able participants } \\
\text { Annotated List Element } \\
\text { (Microsoft Word) }\end{array}$ & $\begin{array}{l}\text { Participants Who Are Blind - } \\
\text { Annotated List Element } \\
\text { (Microsoft Word) }\end{array}$ \\
\hline $\begin{array}{l}\text { 1. Searching } \\
\text { techniques }\end{array}$ & $\begin{array}{l}\text { Scrolling up/down with } \\
\text { the documents scroll bar } \\
\text { - Find / replace menu } \\
\text { access key } \\
\text { - Direct search of menus } \\
\text { with the mouse }\end{array}$ & $\begin{array}{l}\text { - } \begin{array}{l}\text { Searching/reading word- } \\
\text { by-word }\end{array} \\
\text { - Scrolling up/down with } \\
\text { the up/down-arrow keys } \\
\text { - Tab key to parse menu } \\
\text { items } \\
\text { - Find / replace menu } \\
\text { access key } \\
\text { JAWS hot keys } \\
\text { (Insert/Shift/R) to search } \\
\text { MS Word's Revisions' } \\
\text { list }\end{array}$ \\
\hline $\begin{array}{l}\text { 2.Selecting } \\
\text { techniques }\end{array}$ & $\begin{array}{l}\text { Direct manipulation by } \\
\text { selecting and dragging } \\
\text { with the Mouse }\end{array}$ & 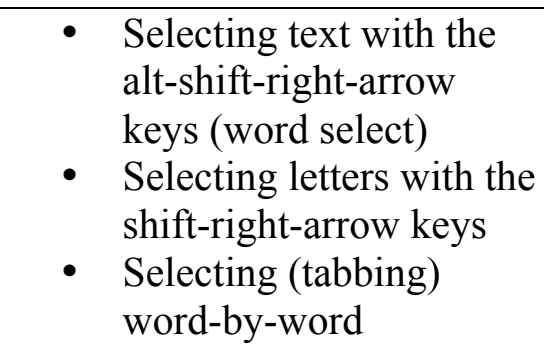 \\
\hline $\begin{array}{l}\text { 3. Editing } \\
\text { techniques } \\
\text { (Same for both } \\
\text { Groups) }\end{array}$ & $\begin{array}{ll}\text { - } & \text { Right-click } \\
\text { (accept/reject list) } \\
\text { - } \\
\text { Copy (control-c)/Paste } \\
\text { (control-v) keys } \\
\text { - } \\
\text { Typing text with a } \\
\text { standard keyboard }\end{array}$ & $\begin{array}{ll}\text { - } & \text { Right-click } \\
\text { (accept/reject list) } \\
\text { - } \\
\text { Copy (control-c)/Paste } \\
\text { (control-v) keys } \\
\text { - } \\
\text { Typing text with a } \\
\text { standard keyboard }\end{array}$ \\
\hline $\begin{array}{l}\text { 4. Reading } \\
\text { techniques }\end{array}$ & $\begin{array}{l}\text { Visually read text and } \\
\text { document structure }\end{array}$ & $\begin{array}{ll}\text { - } & \text { JAWS Screen reader to } \\
\text { read text } \\
\text { - } \\
\text { Braille keyboard to read } \\
\text { text (used in conjunction } \\
\text { with standard keyboard) }\end{array}$ \\
\hline
\end{tabular}




\begin{tabular}{|c|c|c|}
\hline Category & $\begin{array}{l}\text { Visually able participants } \\
\text { Annotated List Element } \\
\text { (Microsoft Word) }\end{array}$ & $\begin{array}{l}\text { Participants Who Are Blind - } \\
\text { Annotated List Element } \\
\text { (Microsoft Word) }\end{array}$ \\
\hline $\begin{array}{l}\text { 5. Accessibility } \\
\text { and usability } \\
\text { issues }\end{array}$ & - None observed & 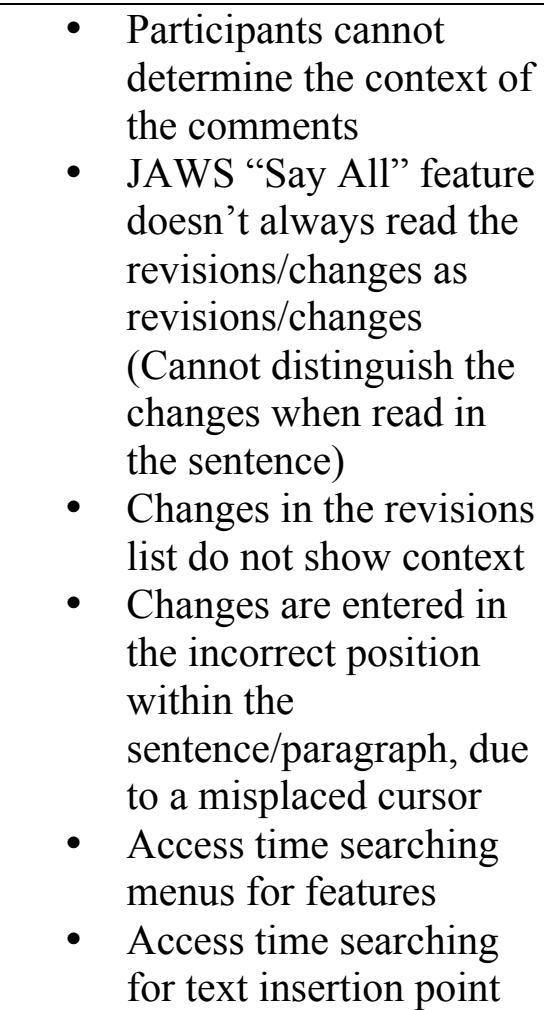 \\
\hline
\end{tabular}

\subsection{Visually able participants}

The visually able participants utilized the computer mouse to interact with the interface. Scroll bars were utilized to view the entire document and to search for specific content. Menu items were searched and accessed with the computer mouse. Specifically, the "find/replace" menu item was selected with the computer mouse to search for specific document content. The computer mouse was utilized for direct manipulation of text such as positioning the cursor to add/edit text, or dragging the cursor to select a word or sentence to edit. Special editing techniques (copy/paste, accept/reject) were accomplished by typing special key press combinations (e.g. Control-c, Control-p) with the computer 
keyboard. Visually able participants were able to visually read the document and its structure. For the visually able participants, no usability issues were identified while utilizing Microsoft Word.

\subsection{Participants Who Are Blind}

For the participants who are blind, the keyboard was the interface device utilized for searching menus and document content. The up/down arrow keys were utilized to move up and down throughout the document. The tab key was utilized to parse and search each word in the document. Additionally, specific key presses were utilized to select menus such as "control-f" for the "find/replace" menu item, and the JAWS hot keys "insert-shift-r" to search the list of revisions of a Microsoft Word document. Special key press combinations (e.g. shift-right-arrow) were utilized to select a specific word, or a complete sentence. The tab key press was utilized to select a word at a time for reading. Special editing techniques (copy/paste, accept/reject) were accomplished by typing special key press combinations (e.g. Control-c, Control-p) with the computer keyboard. Accessibility issues were not identified, but usability issues were identified while utilizing Microsoft Word.

A usability issue identified was related to the participants' inability to identify the context of the comments contained within the document. The participants commented that, "the context of the comments was important in order to understand the purpose of the comment." Another usability issue identified was related to the inability of the JAWS "Say All" feature to read the revisions/changes as revisions/changes, causing the participants who are blind to not be able to identify revisions/changes within a sentence or paragraph. Selecting out revisions/changes into the revisions' list is possible. However, 
the context of the revision is lost when presented in the list of revisions. Access time searching/parsing the Microsoft Ribbon Menu was another usability issue identified. Parsing the menu structure using the keyboard searches the collaborative writing features of Microsoft Word, increasing the access time to select these menu options.

\subsubsection{The Collaborative Writing Experience}

The data in tables 27,28 and 29 shows the perceptions/opinions of the participants with the percentages of agreement and disagreement to each statement in the post-study questionnaire. The data found in these tables can be utilized to determine, if the participants believed that their prior experience helped them complete the tasks and that experience may be important to utilizing collaborative writing features. Or, that the participants thought that the collaborative writing interface was clear to understand and flexible to operate. Or even, if the participants would utilize a particular collaborative writing tool, such as Microsoft Word or Google Docs, in the future. 
Table 27. Microsoft Word - Post-Test Questions/Statements by Visually able participants

\begin{tabular}{|c|c|c|c|c|c|}
\hline Question / Statement & $\mathbf{S A}$ & $\mathbf{A}$ & $\mathbf{N}$ & $\mathbf{D}$ & SD \\
\hline $\begin{array}{l}\text { My years of experience } \\
\text { using a desktop or laptop } \\
\text { computer helped me } \\
\text { complete the tasks. }\end{array}$ & $42.9 \%$ & $57.1 \%$ & $0 \%$ & $0 \%$ & $0 \%$ \\
\hline $\begin{array}{l}\text { My previous knowledge of } \\
\text { the JAWS Screen Reader } \\
\text { helped me complete the } \\
\text { tasks. }\end{array}$ & NA & NA & NA & NA & NA \\
\hline $\begin{array}{l}\text { My experience with } \\
\text { Microsoft Word helped me } \\
\text { complete the tasks. }\end{array}$ & $42.9 \%$ & $57.1 \%$ & $0 \%$ & $0 \%$ & $0 \%$ \\
\hline $\begin{array}{l}\text { The Microsoft Word } \\
\text { interface was clear and } \\
\text { understandable. }\end{array}$ & $28.1 \%$ & $71.9 \%$ & $0 \%$ & $0 \%$ & $0 \%$ \\
\hline $\begin{array}{l}\text { The interactions with } \\
\text { Microsoft Word were } \\
\text { flexible. }\end{array}$ & $42.9 \%$ & $57.1 \%$ & $0 \%$ & $0 \%$ & $0 \%$ \\
\hline $\begin{array}{l}\text { I feel the tasks were easy to } \\
\text { complete. }\end{array}$ & $42.9 \%$ & $42.9 \%$ & $0 \%$ & $0 \%$ & $14.2 \%$ \\
\hline $\begin{array}{l}\text { I am satisfied with the time I } \\
\text { took to complete the tasks. }\end{array}$ & $42.9 \%$ & $57.1 \%$ & $0 \%$ & $0 \%$ & $0 \%$ \\
\hline $\begin{array}{l}\text { I was able to complete the } \\
\text { tasks without any } \\
\text { problem(s). }\end{array}$ & $42.9 \%$ & $42.9 \%$ & $0 \%$ & $0 \%$ & $14.2 \%$ \\
\hline $\begin{array}{l}\text { The collaborative writing } \\
\text { feature of Microsoft Word } \\
\text { improves my performance. }\end{array}$ & $14.3 \%$ & $57.1 \%$ & $28.6 \%$ & $0 \%$ & $0 \%$ \\
\hline $\begin{array}{l}\text { Assuming I have access to } \\
\text { Microsoft Word, I would } \\
\text { use it on a regular basis in } \\
\text { the future when I am doing } \\
\text { similar tasks. }\end{array}$ & $42.9 \%$ & $28.5 \%$ & $14.3 \%$ & $14.3 \%$ & $0 \%$ \\
\hline
\end{tabular}

SA-Strongly Agree, A-Agree, N-Neutral, D-Disagree, SD-Strongly Disagree 
Table 28. Microsoft Word - Post-Test Questions/Statements by Participants Who Are Blind

\begin{tabular}{|c|c|c|c|c|c|}
\hline Question / Statement & $\mathbf{S A}$ & $\mathbf{A}$ & $\mathbf{N}$ & $\bar{D}$ & SD \\
\hline $\begin{array}{l}\text { My years of experience } \\
\text { using a desktop or laptop } \\
\text { computer helped me } \\
\text { complete the tasks. }\end{array}$ & $37.5 \%$ & $62.5 \%$ & $0 \%$ & $0 \%$ & $0 \%$ \\
\hline $\begin{array}{l}\text { My previous knowledge of } \\
\text { the JAWS Screen Reader } \\
\text { helped me complete the } \\
\text { tasks. }\end{array}$ & $25 \%$ & $75 \%$ & $0 \%$ & $0 \%$ & $0 \%$ \\
\hline $\begin{array}{l}\text { My experience with } \\
\text { Microsoft Word helped me } \\
\text { complete the tasks. }\end{array}$ & $37.5 \%$ & $62.5 \%$ & $0 \%$ & $0 \%$ & $0 \%$ \\
\hline $\begin{array}{l}\text { The Microsoft Word } \\
\text { interface was clear and } \\
\text { understandable. }\end{array}$ & $0 \%$ & $25 \%$ & $12.5 \%$ & $62.5 \%$ & $0 \%$ \\
\hline $\begin{array}{l}\text { The interactions with } \\
\text { Microsoft Word were } \\
\text { flexible. }\end{array}$ & $12.5 \%$ & $25 \%$ & $12.5 \%$ & $37.5 \%$ & $0 \%$ \\
\hline $\begin{array}{l}\text { I feel the tasks were easy to } \\
\text { complete. }\end{array}$ & $12.5 \%$ & $37.5 \%$ & $12.5 \%$ & $37.5 \%$ & $0 \%$ \\
\hline $\begin{array}{l}\text { I am satisfied with the time } \\
\text { I took to complete the } \\
\text { tasks. }\end{array}$ & $0 \%$ & $50 \%$ & $12.5 \%$ & $37.5 \%$ & $0 \%$ \\
\hline $\begin{array}{l}\text { I was able to complete the } \\
\text { tasks without any } \\
\text { problem(s). }\end{array}$ & $0 \%$ & $37.5 \%$ & $12.5 \%$ & $50 \%$ & $0 \%$ \\
\hline $\begin{array}{l}\text { The collaborative writing } \\
\text { feature of Microsoft Word } \\
\text { improves my performance. }\end{array}$ & $25 \%$ & $75 \%$ & $0 \%$ & $0 \%$ & $0 \%$ \\
\hline $\begin{array}{l}\text { Assuming I have access to } \\
\text { Microsoft Word, I would } \\
\text { use it on a regular basis in } \\
\text { the future when I am doing } \\
\text { similar tasks. }\end{array}$ & $60 \%$ & $40 \%$ & $0 \%$ & $0 \%$ & $0 \%$ \\
\hline
\end{tabular}

SA-Strongly Agree, A-Agree, N-Neutral, D-Disagree, SD-Strongly Disagree 
All of the participants agreed that their previous experience with computers and Microsoft Word was important in completing the baseline usability study tasks. Additionally, all of the participants who are blind agreed that their previous experience with the JAWS Screen Reader was important in completing the baseline usability study tasks. Therefore, prior experience was considered important to completing the baseline usability tasks.

All of the visually able participants agreed (71.9\%) or strongly agreed (28.1\%) that Microsoft Word's interface was clear and understandable. Whereas, the majority of participants who are blind disagreed (62.5\%) that Microsoft Word's interface was clear and understandable. The participants who are blind regard Microsoft Word's interface as being unclear and not understandable as opposed to the visually able participants' who all agreed that Microsoft Word's interface was clear and understandable.

In regard to the flexibility of Microsoft Word's interface interactions, all of the visually able participants agreed (57.1\%) or strongly agreed $(42.9 \%)$ that the interactions with Microsoft Word were flexible. However, more than one-third of the participants who are blind disagreed (37.5\%) that the interactions with Microsoft Word were flexible.

All visually able participants and $50 \%$ of the participants who are blind agreed that the tasks were easy to complete and that they were satisfied with the time it took to complete each task. Additionally, the majority of the visually able participants and all of the participants who are blind agreed that the collaborative writing feature of Microsoft Word improved their performance and that they would utilize Microsoft Word in the future to do similar collaborative writing tasks. 
However, fourteen point two percent $(14.2 \%)$ of the visually able participants strongly disagreed that the tasks were easy to complete and that the tasks were completed without any problems. Fourteen point three percent (14.3\%) of the visually able participants disagree that they would utilize Microsoft Word to do similar collaborative writing tasks in the future. The participants who are blind disagreed $(37.5 \%)$ that the tasks were easy to complete, disagreed (37.5\%) that they were satisfied with the time to complete each task, and disagreed (50\%) that they were able to complete the tasks without any problem.

Even with these negative survey results, the majority of participants reported that they would utilize Microsoft Word, the market leader, in the future to do similar tasks and believed that the Microsoft Word interface improved their performance. The participants who are blind identified Microsoft Word as being the most accessible and usable collaborative writing tool, and with some minor improvements for track changes could be the best collaborative writing tool on the market. Therefore, this research had to address the accessibility and usability of the track changes and comment features of Microsoft Word in order to provide a total collaborative writing solution. 
Table 29. Google Docs - Post-Test Questions/Statements by Visually able participants

\begin{tabular}{|l|l|l|l|l|l|}
\hline \multicolumn{1}{|c|}{ Question / Statement } & \multicolumn{1}{c|}{ SA } & A & N & D & SD \\
\hline $\begin{array}{l}\text { My years of experience using a } \\
\text { desktop or laptop computer } \\
\text { helped me complete the tasks. }\end{array}$ & $42.9 \%$ & $57.1 \%$ & $0 \%$ & $0 \%$ & $0 \%$ \\
\hline $\begin{array}{l}\text { My previous knowledge of the } \\
\text { JAWS Screen Reader helped } \\
\text { me complete the tasks. }\end{array}$ & NA & NA & NA & NA & NA \\
\hline $\begin{array}{l}\text { My experience with Google } \\
\text { Docs helped me complete the } \\
\text { tasks. }\end{array}$ & $0 \%$ & $71.4 \%$ & $14.3 \%$ & & $14.3 \%$ \\
\hline $\begin{array}{l}\text { The Google Docs interface was } \\
\text { clear and understandable. }\end{array}$ & $14.3 \%$ & $14.3 \%$ & $14.3 \%$ & $57.1 \%$ & $0 \%$ \\
\hline $\begin{array}{l}\text { The interactions with Google } \\
\text { Docs were flexible. }\end{array}$ & $14.3 \%$ & $14.3 \%$ & $0 \%$ & $71.4 \%$ & $0 \%$ \\
\hline $\begin{array}{l}\text { I feel the tasks were easy to } \\
\text { complete. }\end{array}$ & $14.3 \%$ & $28.6 \%$ & $0 \%$ & $42.9 \%$ & $14.3 \%$ \\
\hline $\begin{array}{l}\text { I am satisfied with the time I } \\
\text { took to complete the tasks. }\end{array}$ & $14.3 \%$ & $57.1 \%$ & $0 \%$ & $28.6 \%$ & $0 \%$ \\
\hline $\begin{array}{l}\text { I was able to complete the tasks } \\
\text { without any problem(s). }\end{array}$ & $14.3 \%$ & $28.6 \%$ & $0 \%$ & $42.9 \%$ & $14.3 \%$ \\
\hline $\begin{array}{l}\text { The collaborative writing } \\
\text { feature of Google Docs } \\
\text { improves my performance. }\end{array}$ & $14.3 \%$ & $57.1 \%$ & $0 \%$ & $14.3 \%$ & $14.3 \%$ \\
\hline $\begin{array}{l}\text { Assuming I have access to } \\
\text { Google Docs, I would use it on } \\
\text { a regular basis in the future } \\
\text { when I am doing similar tasks. }\end{array}$ & $14.3 \%$ & $42.9 \%$ & $0 \%$ & $28.6 \%$ & $14.3 \%$ \\
\hline SA-Strongy Ag & & & & & \\
\hline
\end{tabular}

SA-Strongly Agree, A-Agree, N-Neutral, D-Disagree, SD-Strongly Disagree

When visually able participants evaluated their experience utilizing Google Docs, there was a shift from positive feedback provided for Microsoft Word (Table 27) to negative feedback for Google Docs (Table 29). There was a shift from agreement/strong agreement to disagreement/strong disagreement. The majority of visually able participants' disagreed (57.1\%), when asked if the Google Docs' interface was clear and 
understandable, and disagreed (71.4\%), when asked if the Google Docs' interface was flexible. Participant disagreement (42.9\%) and strong disagreement (14.3\%) occurred when the visually able participants responded to the statements, "I feel the tasks were easy to complete," and "I was able to complete the tasks without any problems." This seems to imply that the visually able participants felt that they had difficulties and problems completing the usability study tasks when interacting with the Google Docs interface. The issues observed by the researcher while the visually able participants interacted with Google Docs was the time for the interface to display any updates and the lag time when searching and editing text. Another area that presented issues for the visually able participants was identifying individual changes, since the changes are time recorded as a group with Google Docs and not individually recorded as with Microsoft Word's track changes.

The negative responses in regard to Google Docs versus Microsoft Word can be attributed in part to the visual participant's lack of experience with Google Docs. For example, in Table $27,28.6 \%$ of the visually able participants were either neutral or strongly disagreed with the statement, "My experience with Google Docs helped me complete the tasks."

The visually able participants' disagreed (14.3\%) and strongly disagreed (14.3\%) that Google Docs improved their collaborative writing performance, and disagreed $(28.6 \%)$ and strongly disagreed $(14.3 \%)$ that Google Docs would be their choice when collaborative writing in the future. 
Examining the collective responses of visually able participants in regard to Microsoft Word and Google Docs, it appears that the visually able participants prefer Microsoft Word to Google Docs for collaborative writing.

\subsubsection{Perception of the Collaborative Writing Experiences}

The likes, dislikes, and suggestions on how to improve the collaborative writing experience data were collected from the answers to the open-ended questions in the poststudy questionnaire. Tables 30, 31 and 32 presents the feedback in regard to the Microsoft Word's interface and Google Docs' interface by visually able and participants who are blind respectively.

Table 30. Feedback from Visually able participants regarding Microsoft Word

\begin{tabular}{|l|l|}
\hline \multicolumn{1}{|c|}{ Category } & \multicolumn{1}{c|}{ Sample Comments } \\
\hline Positive & $\begin{array}{l}\text { Each change was presented visually. } \\
\text { - } \quad \begin{array}{l}\text { Each change could be accessed independently by searching for } \\
\text { the next or previous change. }\end{array} \\
\text { - I like how the collaborative writing isn't synchronous. This } \\
\text { allows me to easily distinguish the changes I made from my } \\
\text { partner's changes made at different times. } \\
\text { Changes are easily tracked between multiple people. Each } \\
\text { change is tracked separately - not at the 'last save' level. }\end{array}$ \\
\hline Negative & $\begin{array}{l}\text { Can be difficult to understand the change when multiple changes } \\
\text { occur to the same line of text. Have to accept the change to see the } \\
\text { result of the change. }\end{array}$ \\
\hline $\begin{array}{l}\text { Suggested } \\
\text { Improvement }\end{array}$ & Allow changes to be reviewed, as they would look if accepted. \\
\hline
\end{tabular}


Table 31. Feedback from Participants Who Are Blind regarding Microsoft Word

\begin{tabular}{|c|c|}
\hline Category & Sample Comments \\
\hline Positive & $\begin{array}{l}\text { It is the most accessible solution I've found for collaborative } \\
\text { projects, not perfect but better than others. } \\
\text { There are choices to accept or delete changes and they are } \\
\text { accessible with JAWS. }\end{array}$ \\
\hline Negative & $\begin{array}{l}\text { - I always struggle with accessing comments. } \\
\text { - Not familiar with the ribbon menus and finding the track } \\
\text { changes menu items takes time, which can take some getting } \\
\text { used to. } \\
\text { - It takes some time to understand how track changes works and } \\
\text { what parts are spoken. It takes time to figure out where and } \\
\text { what the comments are. } \\
\text { - JAWS could be more precise with some of the messages, such } \\
\text { as not saying, "inserted" in the middle of a section of inserted } \\
\text { text. }\end{array}$ \\
\hline $\begin{array}{l}\text { Suggested } \\
\text { Improvement }\end{array}$ & $\begin{array}{l}\text { - Screen readers need to be able to give better context when } \\
\text { identifying comments and revisions. } \\
\text { - Maybe some clues from the system about what action is taken, } \\
\text { whether something is actually inserted, accepted or deleted. } \\
\text { - Since, I had some problems with reading deleted text and } \\
\text { comments. The user needs to be able to review the text in two } \\
\text { ways, without and with the changes. }\end{array}$ \\
\hline
\end{tabular}

When examining the collaborative writing features of Microsoft Word, visually able participants (Table 30) positively commented on the visual nature of the document changes and that each change is independent and traceable by collaborator. The visually able participants commented that it was easier to collaborate asynchronously and in a serial manner, where each collaborator took their turn editing the same document, as opposed to purely synchronous. Clearly, the visually able participants prefer asynchronous collaboration to synchronous collaboration when composing or editing documents. Participant who are blind (Table 31) positively commented that Microsoft 
Word was the most accessible collaborative writing application available, and that changes to documents are accessible and can be accepted or rejected. Both visually able participants and participants who are blind viewed Microsoft Word as accessible and usable in regard to track changes and collaborative writing.

On the negative side, a visually able participant (Table 30) commented that, "it could be difficult to understand multiple changes, when made to a single sentence." Accepting the changes was the solution to understanding all of the changes in specific context (Sentence or paragraph). A participant who is blind (Table 31) commented, " $I$ am not familiar with the ribbon menus and finding the track changes menu items takes time and experience." Both visually able participants and participants who are blind, considered track changes difficult to distinguish and understand unless they were presented as accepted, and that the comments were also difficult to distinguish and understand unless the comment's context was known and the comments were distinguishable from the text content.

As far as improving the interface, visually able participants (Table 30 ) commented that allowing changes to be reviewed as if they had already been accepted would be a good feature. Participants who are blind (Table 31) suggested improving Microsoft Word's interface by being precise in distinguishing text from comments, and providing the context of inserted/deleted changes and comments by providing paragraph content with the changes and comments. Additionally, participants who are blind would like to be notified when text is added/deleted or accepted/rejected, and have the ability to review the changes in their accepted form. 
Table 32 presents the comments from visually able participants while completing the baseline usability study using Google Docs' interface. Participants who are blind did not interact with Google Docs, so there is no data to report for that study group.

Table 32. Feedback from Visually able participants regarding Google Docs

\begin{tabular}{|c|c|}
\hline Category & Sample Comments \\
\hline Positive & $\begin{array}{l}\text { The changes were included in the document as completed. } \\
\text { - You are able to revise work with others in real-time. Able } \\
\text { to understand changes and revisions to your work while } \\
\text { collaborating with someone in real time. } \\
\text { Documents live on the Internet - it acts like a central file } \\
\text { server. }\end{array}$ \\
\hline Negative & $\begin{array}{l}\text { Difficult to determine the changes. Had to access the } \\
\text { revisions, in order to see the changes. Changes were hard to } \\
\text { identify. Had to access another area to see the changes. } \\
\text { The application is horrible. I'll never use it again. } \\
\text { Operations were slow and worse, unpredictably slow. I } \\
\text { needed to paste/edit multiple times before I saw it take } \\
\text { effect on the screen. Would not work with IE8 - switched } \\
\text { to Firefox mid-exercise. I would rather send iterations } \\
\text { though the mail then use this again. } \\
\text { You could get confused between you and your partner if } \\
\text { you're editing the same block of text in real-time. } \\
\text { Interactions such as typing at the same time as the others } \\
\text { during a co-editing session can be confusing. }\end{array}$ \\
\hline $\begin{array}{l}\text { Suggested } \\
\text { Improvement }\end{array}$ & $\begin{array}{l}\text { Keep collaborative process simple. One person edits or } \\
\text { revises the document one by one, not all at once. It allows } \\
\text { for a smoother transition of ideas instead of multiple ideas } \\
\text { all at once. } \\
\text { Possibly display the changes when you are in a specific } \\
\text { paragraph. Toggle display changes/ do not display changes } \\
\text { by key press. } \\
\text { - Add a Microsoft Word-like panel to highlight the changed } \\
\text { portions of the text. }\end{array}$ \\
\hline
\end{tabular}


When examining the collaborative writing features of Google Docs, visually able participants positively commented that the changes are presented as accepted, so they were easy to review within the sentence or paragraph, collaborative writing is in realtime, and the edited documents are stored in an internet file system.

On the negative side, a visually able participant commented that, "it was difficult to identify the changes, since they appeared the same as unchanged text." The operation of the Google Docs' interface was slow and time delays between tasks made the interface unacceptable for use. A participant commented, "The application is horrible. I'll never use it again. Operations were slow and worse, unpredictably slow. I needed to pasteledit multiple times before I saw it take effect on the screen." In order to see the changes, you have to access the revisions list and scroll through several iterations of the document to review changes. A participant commented that, "Changes were hard to identify. I had to access another area to see the changes." Collaborating with other participants in realtime can be even more difficult, if the collaborators are editing the same block (sentence or paragraph) of text. A participant commented that, "Interactions such as typing at the same time as the others during a co-editing session can be confusing."

This kind of experience leads to the preference of visually able participants on using an asynchronous editing tool rather than trying to edit the same document section simultaneously by multiple group members. Therefore, when being asked how to improve Google Docs' interface, visually able participants (Table 32) commented that each collaborator should edit the document one by one and not concurrently, and the software interface should provide indications of change. 


\subsubsection{Implications}

The usability studies highlighted key areas where persons who are blind had difficulty completing certain tasks. The participants who are blind considered track changes difficult to distinguish and understand unless they accept all the changes before reviewing the document. The comments were also difficult to distinguish and understand because the context of the comments was not clearly presented. The participants commented that the tasks were not easy to complete, that they were dissatisfied with the time they took to complete the tasks and that they were able to complete the tasks without any problems. Based on the data analysis for the usability, it is clear that providing the context for the edits is a key factor for successfully utilizing track changes and comments features.

\subsubsection{Conclusion}

In regard to Microsoft Word, there were issues related to the list of revisions. The inability of participants who are blind to understand the context of revisions and comments, and their inability to understand the presence of these revisions and comments within the sentence and paragraph was a common concern voiced by these participants. In order to present the context of comments and revisions, additional interface tools should be added to present the sentence or paragraph where a change or comment exists.

In order to improve the awareness of the revisions and comments, two possible views should be presented: one view without the revisions included (prior/original view); and, another view with the revisions (resulting view).

While interacting with Microsoft Word, participants who are blind could not directly access menu items for accepting or rejecting changes, finding the next change, 
and finding the next comment. The participants who are blind had to parse the menu structure with the tab key and listen for the proper menu item. Direct key press access would improve the access time to these features and should be programmed directly into Microsoft Word.

In regard to Google Docs, there were issues related to the slow response and repainting of the interface. The slow response time and repainting the interface would be improved by improving the network bandwidth and the amount of memory of the computer. This issue is more a result of environmental factors, such as the network and computer memory and not necessarily an interface design issue. Revisions are saved in time increments, so the visually able participants could not accept or reject a specific revision, but had to accept or reject a group of revisions. To improve the Google Docs interface in regard to revisions, each revision should be saved independent from other revisions.

The usability studies concluded that the participants who are blind took almost twice as much time in several collaborative writing tasks when compared with the visually able participants. Given this result, additional participants who are blind participated in usability studies to validate the baseline quantitative measures. What was discovered is that as the experience level of utilizing Microsoft Word collaborative writing features drops, so does the ability to complete certain collaborative writing tasks related to revisions and comments. This was evident by the $62 \%$ task completion rate while searching, accepting and rejecting revisions in a Microsoft Word document.

Based on these results, it was decided that an improvement to the Microsoft Word interface was necessary to improve access to revisions and comments and to provide the 
context of the revisions and comments. The next section will present the details of the proposed solution for an accessible Microsoft Word interface. 


\subsection{The Prototype Design}

Based on the data analysis from the baseline usability study (Schoeberlein \& Wang, 2012), it is clear that providing the context for the revisions and comments is the key for persons who are blind to successfully utilize the track changes and comments features of Microsoft Word. Since Microsoft Word is still one of the most frequently used editing tools in the work place, the solution needs to be able to work with Microsoft Word seamlessly. The participants who are blind suggested the following improvements to make the application more accessible and usable:

1. Clearly differentiate the text from the comments;

2. Provide the context of proposed changes and comments by providing paragraph content with the changes and comments;

3. Notify the user where the change is proposed; and,

4. Give the ability to review changes in their accepted form.

\subsubsection{Microsoft Word Application Add-In}

Since Microsoft Word provided the majority of editing features, a proposed Microsoft Word Add-In was developed to showcase the importance of providing context of revisions and comments, and to provide the ability to directly access these features without parsing the Ribbon Menu. A Microsoft Word Application Add-In (Word AddIns) is a tool or application that is developed to integrate with Microsoft Word in order to expand or enhance the features of Microsoft Word. In this case, to improve the access and use of track changes and comments collaborative writing features included with Microsoft Word. This Add-In would be set at the application level, allowing the proposed features to be available for any Microsoft Word document. In order to access the 
revisions and comments, the Microsoft Word Paragraphs ("MSDN Paragraphs Collection," 2013), Revisions ("MSDN Revisions Collection," 2013) and Comments ("MSDN Comments Collection," 2013) Collections are accessed to present the revisions and comments within the context of their paragraphs.

\subsubsection{Microsoft Word Add-In Features Designed/Implemented}

The proposed Microsoft Word add-in is compatible with different versions of Microsoft Word and provides the following features:

1. Provide a comprehensive view of all revisions and comments.

2. Present the context (paragraph or sentence) of each comment and revision.

3. Identify each revision as an addition, a deletion, or a change.

4. Present each comment separately.

5. Select/highlight each revision/comment in a series.

6. Providing direct access to the features of the Add-In through a Microsoft Word Command Bar.

7. Provide the "Final" view, as if the revisions are applied, for each paragraph or sentence.

8. Provide the "Revision" view, with the in-line revisions, for each paragraph or sentence.

9. Compatible with JAWS 13.

10. Provide optional audio feature as an alternative to JAWS. 


\subsubsection{Comprehensive View}

To provide a comprehensive view of the revisions and comments, the Microsoft Word Add-In presents an overview of the total number of paragraphs or sentences, along with the total number of revisions and comments (Figure 19). This overview is necessary for any person to know immediately whether they have revisions or comments to review.

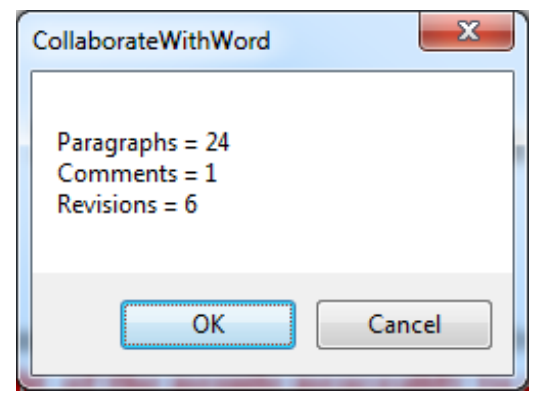

Figure 19 Overview

\subsubsection{Present the context (paragraph or sentence) of each comment and revision}

Based on the comments of the participants of the baseline usability study (Schoeberlein \& Wang, 2012), it was determined that the context of a revision or comment was necessary to the understanding of the revision or comment being reviewed. However, Microsoft Word does not properly present the context of revisions or comments, making review difficult for persons who are blind (Schoeberlein \& Wang, 2012). In order to provide the proper context for revisions and comments, the paragraph (Figure 20) or the sentence containing the revision of comment is presented. 


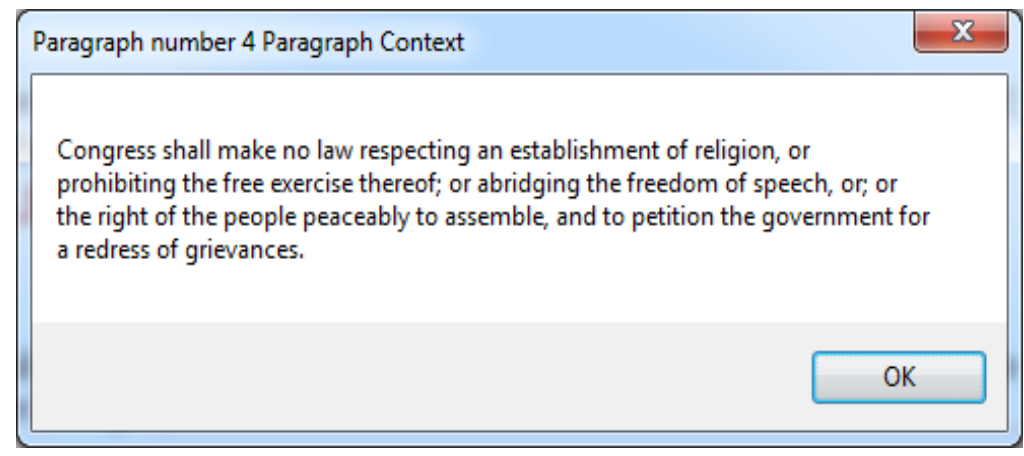

Figure 20 Paragraphs or Sentence Context

10.3.2.3 Identify each Revision as an Addition, a Deletion, or a Change

In addition to the context of a revision or comment, it is essential that the type of revision be identified. Figure 21 presents an inserted revision.

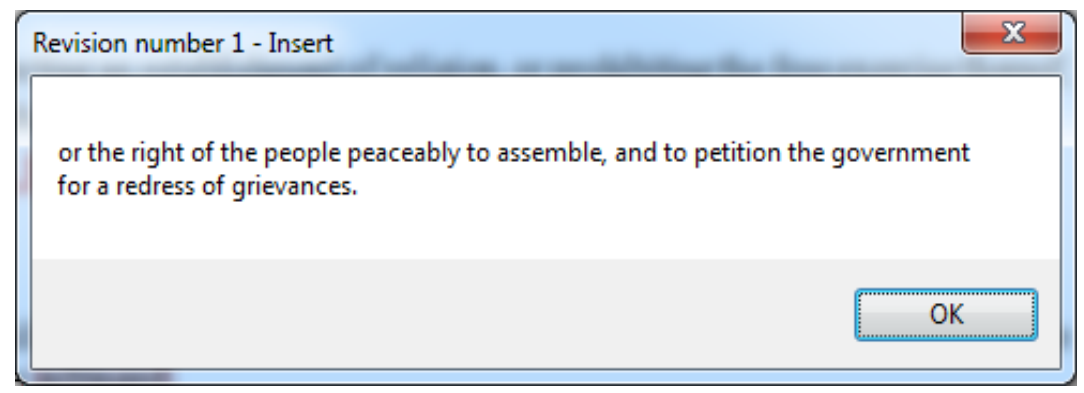

Figure 21 Insert Revision

\subsubsection{Present each comment separately}

The comments in the document will be presented one by one when the user requests it.

\subsubsection{Select/highlight each revision/comment in a series}

In order to act upon any revision or comment, the revision or comment must be selected by the Microsoft Word Add-In (Figure 22). Once selected (highlight in blue), the revision can be accepted or rejected. The selected comment can be deleted. While 
reviewing the revisions, the Add-In prototype will automatically highlight the corresponding sentences to allow the user to accept/reject the proposed change when they are ready to take the action.

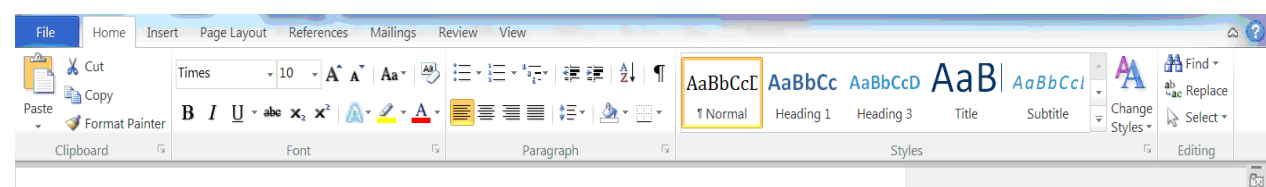

Amendments of the United States Constitution

\section{Amendment I}

Congress shall make no law respecting an establishment of religion, or prohibiting the free exercise thereof; or abridging the freedom of speech, or; or the right of the people peaceably to assemble, and to petition the government for a redress of grievances.

\section{Amendment II}

| A well regulatedwell-regulated militia, being necessary to the security of a free state, the right of the people to keep and bear arms, shall not be infringed.

Figure 22 Revision View with selected revision (highlight in blue)

\subsubsection{Provide Direct Access.}

Since accessing the Microsoft Word Ribbon Menu was a usability issue for the participants who are blind (Schoeberlein \& Wang, 2012), and in order to provide seamless interface integration of the Microsoft Word Application Add-In ("Word AddIns," 2013) with Microsoft Word, the Microsoft Word Command Bar ("MSDN Microsoft Command Bar," 2013) was utilized to add features necessary to provide improved access and use of the track changes and comments features of Microsoft Word. 


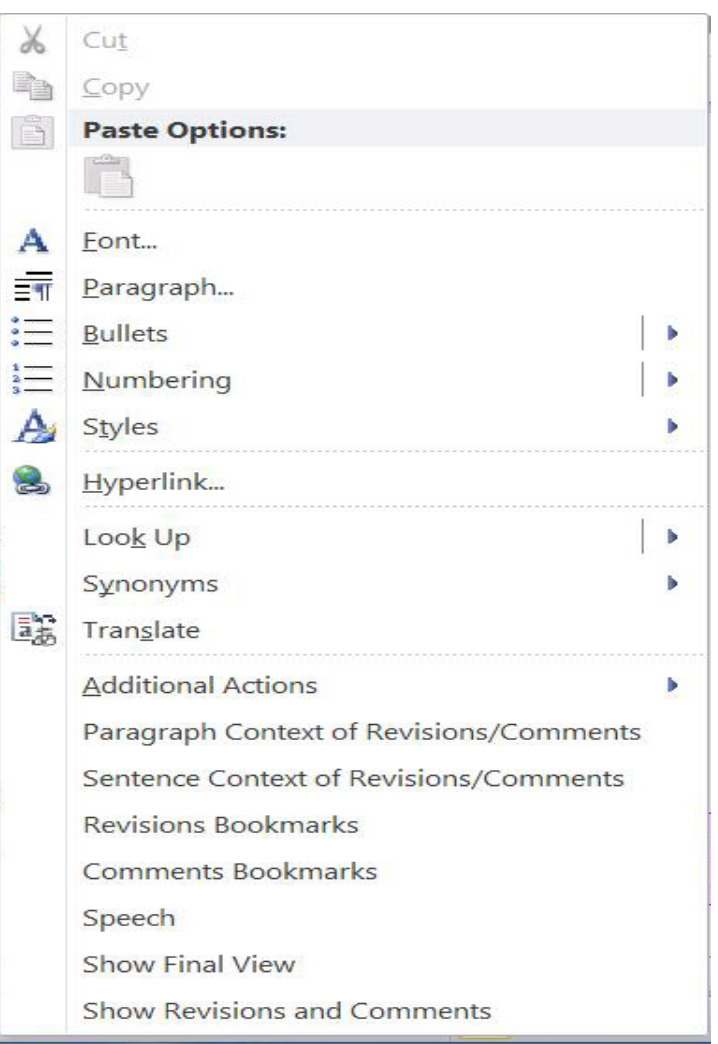

Figure 23 Microsoft Word Command Bar

The Microsoft Word Command Bar would allow the end-user the ability to avoid accessing the Microsoft Word Ribbon Menu by simply right-clicking anywhere on the document to access the Command Bar, to directly access the application's features.

\subsubsection{Revision View versus Final View.}

The baseline usability study participants prefer to have alternative views of the paragraphs and sentences with revisions and comments (Schoeberlein \& Wang, 2012). Users should be able to toggle between two views depending on their own preference: to review the content with the revisions (Figure 22), or to review the content after the revisions are applied (Figure 24). 


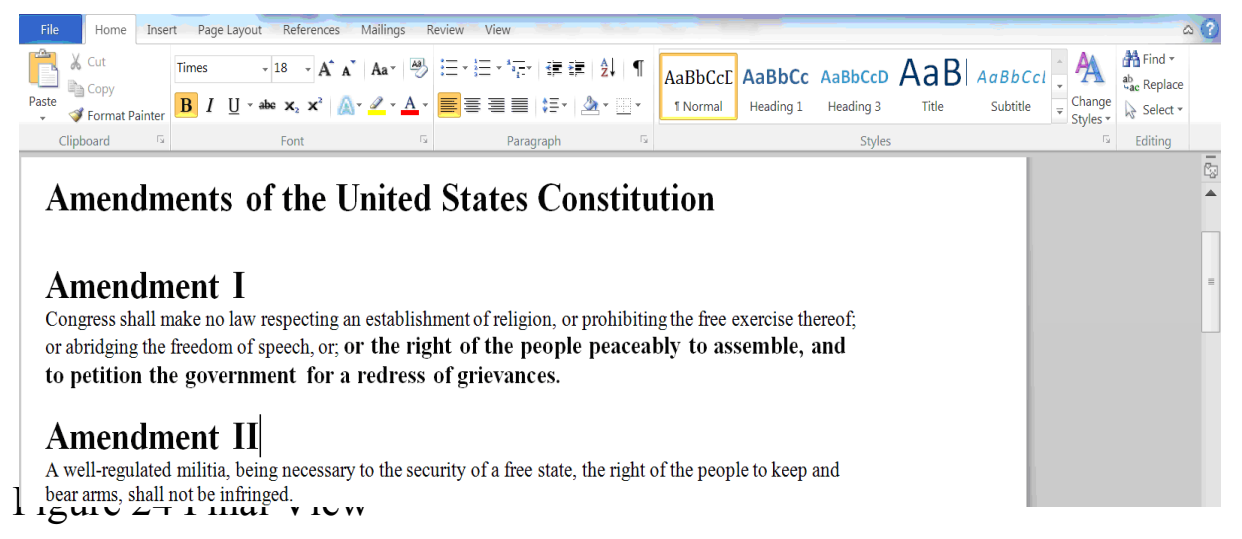

\subsubsection{JAWS and Built-In Audio}

The JAWS Screen Reader ("Freedom Scientific JAWS," 2008) is the most commonly used output mechanism by persons who are blind in order to interface with any computer application. Therefore, the developed Microsoft Word Add-In and Microsoft Word Command Bar have to be compatible with the JAWS Screen Reader. The Microsoft Word Add-In and the Microsoft Word Command Bar and the message boxes presented by the Add-In have been validated to work seamlessly with the JAWS Screen Reader.

Alternatively, a Microsoft Speech API was included in the development of the Microsoft Word Add-In, to provide audio output for persons who did not have access to the JAWS Screen Reader. No content is displayed on the interface, only the audio content representing the messages boxes that would otherwise display for the JAWS Screen Reader. 


\subsubsection{Microsoft Word Add-In Benefits}

The proposed Microsoft Word Add-In provides many benefits to the users. Since the Add-In is a component of Microsoft Word, it is seamlessly integrated into Microsoft Word. Users do not need any prior knowledge to utilize the Command Bar, since they can simply right-click anywhere on a document to have direct access to the commands. With this direct access feature, there is no need to memorize complex key stoke combinations when working on the document. Therefore, the cognitive load is reduced to prevent overload. Since the Add-In is applied at the application level, it works with all Microsoft Word documents. It provides descriptive information regarding any comments/revisions including their sequence as well as type and content of the revisions. In addition, users are presented with an overview of the document and context of the revision/comments so that users are aware of the context of any action. The Add-In can be upgraded to different versions of Microsoft Word and is compatible with the JAWS screen reader. This eliminates the need for additional investment for the application to be accessible. 


\subsection{Pilot Usability Study}

The pilot usability study was conducted to examine the Microsoft Word Add-In prototype in order to test the concept of the command bar interface, to validate its compatibility with the JAWS Screen Reader and Microsoft Word, and to improve and tweak the interface before the examination in the final usability study. The pilot usability study utilized a guided expert-based testing (Lazar et al., 2010). The participant selected, who is blind with no residual vision, was a quasi-expert with Microsoft Word, the JAWS Screen Reader, accessibility and usability.

The next sections present the details of the pilot usability, and include the selection criteria for the participant, the environment configuration, the usability study procedure and tasks, the data collection methods, the results, implications and conclusion.

\subsubsection{Participant}

One quasi-expert participant, who was experienced with Microsoft Word and the JAWS screen reader, was recruited from the membership of the National Federation of the Blind (NFB), in order to pilot test the Microsoft Word Add-In prototype, the usability study procedure and instruments. The participant was blind, with no residual vision, and was considered an experienced user of Microsoft Word (6 to 10 years) and the JAWS Screen Reader (11 to 20 years). The choice of utilizing one experienced participant was to validate the basic operations of the prototype and not intended as a full-blown usability study. The pilot usability study was intended to prove the soundness of the prototype design and the usability study procedure and measurements. 


\subsubsection{Environment Configurations}

The computer configuration consisted of a Dell Latitude E6400 Laptop Computer running Windows 7 Enterprise. The installed applications consisted of Microsoft Word 2010, JAWS version 13, and the developed Microsoft Word Ad-In. The external hardware consisted of a standard keyboard.

\subsubsection{Procedure}

The usability study session began with a brief introduction of the purpose of the study, followed by the signing of an informed consent form (Appendix G). The participant completed a pre-study questionnaire to collect background information about the participant (Appendix H). Following the completion of the pre-study questionnaire, the participant was introduced to the functionality of the Microsoft Word Add-In prototype. The usability study session was conducted based on the tasks to complete from the task lists (Appendix I - MS Word), utilizing the Microsoft Word Add-In prototype. The participant examined the document (Appendix J), “Amendments of the United States

\section{Constitution."}

During the usability study session, the researcher recorded task performance (complete or incomplete), task completion time (time to complete) directly next to each task on the task list. Audio, video and researcher notes were collected.

Following the usability study, a brief post-study questionnaire was collected on the usability study experience and the opinions in regard to the developed Microsoft Word Add-In (Appendix D). The usability study sessions ran for 90 minutes. 


\subsubsection{Tasks}

The collaborative writing tasks (Table 24) included tasks associated with searching, and accepting and rejecting changed text throughout the document.

\subsubsection{Data Collection}

The data sources from the usability study session are the same as the baseline usability study and included the pre-study and post-study questionnaires, the task list, the researcher's notes, and the audio and video recordings. The researcher and an assistant coded the qualitative data using the Grounded Theory (2009) and validated agreement of the qualitative data using Cohen's Kappa (2010). Agreement was considered acceptable at $70 \%$ agreement among the coders. Forty-seven cases were identified with the coders agreeing on $86.4 \%$ of these cases, providing evidence of acceptable agreement and reliability between the coders. 
10.4.6 Data Analysis and Results

\subsubsection{Demographics}

Demographical data was collected from the response collected from the pre-study questionnaire. The participant of the pilot usability study was a male in his 40's, who had 11 to 20 years computer experience, used the computer daily at work, which had 11 to 20 years' experience with the JAWS screen reader, and used Microsoft Word daily for the past 6 to 10 years. Based on his experience with Microsoft Word and the JAWS screen reader, he was considered a quasi-expert user.

\subsubsection{Task Completion Percent and Mean}

During the usability study session, the begin time and end time was recorded for each task in the task list. They are utilized to determine a time range that could be quantified as a task completion time. The participant completed all tasks of the pilot usability study as follows:

1. Search for all changes (30 seconds);

2. Accept a change (45 seconds);

3. Reject a change (50 seconds); and,

4. Accept/Reject all remaining changes one-by-one (75 seconds). 


\subsubsection{Analysis of the audio/video recordings}

Forty-seven cases were identified with the coders agreeing on $86.4 \%$ of these cases, providing evidence of acceptable agreement and reliability between the coders.

Table 33 presents the categories and examples identified from the participant transcript from the usability study audio/video recording collected during the examination of the Microsoft Word Add-In Prototype.

Table 33. Categories and Sample Data/Comments

\begin{tabular}{|l|l|}
\hline \multicolumn{1}{|c|}{ Category } & \multicolumn{1}{c|}{ Sample Data/Comments } \\
\hline 1. Improved & $\begin{array}{l}\text { Allowing simple interactions with the interface such as } \\
\text { right-clicking on the interface to display the Microsoft } \\
\text { Word Command Bar }\end{array}$ \\
- $\begin{array}{l}\text { Providing the entire paragraph context to be read by a } \\
\text { screen reader }\end{array}$ \\
- $\begin{array}{l}\text { Providing comprehensive messages, such as, "Number of } \\
\text { revision are X" }\end{array}$ \\
$\begin{array}{l}\text { Providing a cancel (esc key press) to exit the logic cycle } \\
\text { of the Microsoft Word Add-In } \\
\text { Providing a Ok (enter key press) to continue the logic } \\
\text { cycle of the Microsoft Word Add-In }\end{array}$ \\
- $\begin{array}{l}\text { Fully integrating the Microsoft Word Add-In within } \\
\text { Microsoft Word and not as a separate program } \\
\text { Providing context which should improve understanding of } \\
\text { the revision(s) } \\
\text { Providing the sentence with the revisions and without the } \\
\text { revisions }\end{array}$ \\
\hline - $\begin{array}{l}\text { It might make it easier to say, "There are 24 paragraphs, } \\
\text { there are X revisions and X comments." }\end{array}$ \\
needed
\end{tabular}




\begin{tabular}{|c|c|}
\hline Category & Sample Data/Comments \\
\hline $\begin{array}{l}\text { 3. Current } \\
\text { features }\end{array}$ & $\begin{array}{ll} & \text { Show Final View } \\
\text { - } & \text { Show Revisions View } \\
\text { - } & \text { cofaults to show Revisions / Comments with paragraph } \\
\text { - } & \text { Speech } \\
\text { - } & \text { Integrated with JAWS } \\
\text { - } & \text { Integrated with Microsoft Word }\end{array}$ \\
\hline $\begin{array}{l}\text { 4. Previous } \\
\text { issues }\end{array}$ & $\begin{array}{l}\text { - "JAWS - so hard to identify track changes." } \\
\text { "I use to have to ask people to not use track changes. Take } \\
\text { the paragraphs you want to change and post them into an } \\
\text { email message. Make the changes and explain your } \\
\text { changes." } \\
\text { - "Not fair to ask others for special edits to the document." } \\
\text { - "Collaboration is one area that manufacturers have not } \\
\text { done well." } \\
\text { - I do not know any blind person who is ever happy when } \\
\text { they have to deal with track changes." }\end{array}$ \\
\hline $\begin{array}{l}\text { 5. Benefits of the } \\
\text { Add-In prototype }\end{array}$ & $\begin{array}{l}\text { - "Have all of the options; just look at the revisions } \\
\text { directly." } \\
\text { - "Doorway to independence." } \\
\text { - "Using this add-in, I would not have to make special } \\
\text { requests (email changes) of people." }\end{array}$ \\
\hline
\end{tabular}

10.4.6.4 The Collaborative Writing Experience

The participant agreed that his prior experience with computers and Microsoft Word helped him to complete the pilot usability study tasks, but he was neutral as to the effect his prior experience with JAWS had on his completing the tasks. The JAWS screen reader simply read whatever was presented, and there was no need to utilize special key presses associated with JAWS, so this may explain his response to the affect JAWS played. The JAWS Screen Reader speed setting ranges from 40 (slow) to 150 (fast). For this study the JAWS Screen Reader was set to "Eloquence" at the default speed setting of 57 , which is a normal speech pace. The default speed setting was used for all participants of this study. Adjustments were not made to increase or decrease the voice speed. 
The participant of the pilot usability study examined the Microsoft Word Add-In prototype and responded to a post-survey in regard to his experience. When examining the collaborative writing features of the Microsoft Word Add-In, the participants "agreed" that the Microsoft Word Add-In interface was clear and understandable, and "strongly agreed" that the Microsoft Word Add-In interface was flexible and easy to use, improved performance, and enable tasks to be completed without any problems. Further, the participant "strongly agreed" that he was satisfied with the time he took to complete the study tasks, and that he would utilize the Microsoft Word Add-In interface in the future on a regular basis.

\subsubsection{Perceptions/Feedback Collected on Collaborative Writing Experiences}

The participant reported that the Microsoft Word Add-In interface "helps place the revision in context and identifies exactly what is being changed. It also provides easier access to the comments than just the screen reader." He suggested "some clarification of language on some menu options and message boxes" as improvements to the Microsoft Word Add-In interface. His suggestions included saying, "There are 24 paragraphs, there are $\mathrm{X}$ revisions and $\mathrm{X}$ comments." Instead of the original prompt, "Paragraphs $=24$, Revisions $=X$, Comments $=X . "$ The suggested language does improve the understanding of the content presented. 


\subsubsection{Implications}

Based on the results of the Pilot Usability Study, the Microsoft Word Add-In prototype enhances the track changes and comment features of Microsoft Word.

Additional research is necessary. 


\subsubsection{Conclusion}

The Pilot Usability Study was conducted with a participant who is blind who was considered a quasi-expert user of Microsoft Word and the JAWS Screen Reader. The participant successfully completed the four tasks and stated that he would utilize the Microsoft Word Add-In prototype when doing similar tasks. Additionally, the Microsoft Word Add-In prototype was considered acceptable and proved to be compatible with the JAWS Screen Reader and Microsoft Word. Therefore, it is concluded that the Microsoft Word Add-In prototype should be further examined with additional usability studies in order to fine-tune and improve the interface.

The next section will present the enhancements to the Microsoft Word Add-In prototype. 
10.5 Microsoft Word Add-In Prototype Pilot Enhancements

As a result of the pilot usability study participant's suggestions for improving the Microsoft Word Add-In Prototype, the following enhancements were included with the interface. The participant suggested that it might make it easier to say, "There are 24 paragraphs, there are X revisions and X comments." Figure 25 illustrates this feature change.

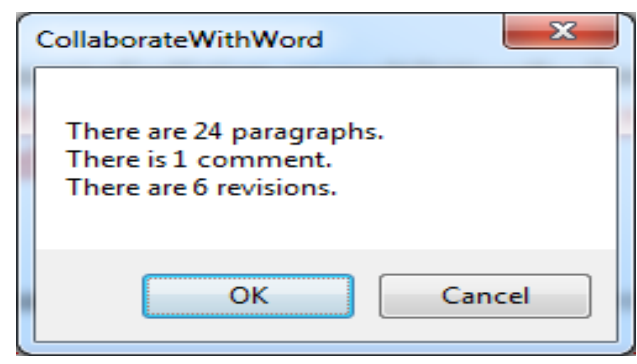

Figure 25 Overview of the document

The participant suggested changes to the text of the Command Bar Options to, "Set view to revisions" and "Set view to final" for two of the Microsoft Word Command Bar options (Figure 26), to clarify their meanings. Previously, the language for the Microsoft Word Command Bar options was, "Revision View" and "Final View." Having the verb "set" clarifies the intention of the options.

Additional Actions
Paragraph Context of Revisions/Comments
Sentence Context of Revisions/Comments
Revisions Bookmarks
Comments Bookmarks
Speech
Set View to Final
Set View to Revisions

Figure 26 "Set View to Final" and "Set View to Revisions" Command Bar Options 


\subsection{Final Usability Study}

Once the pilot review was completed and the Microsoft Word Add-In prototype was considered acceptable and proved to be compatible with the JAWS Screen Reader and Microsoft Word, the usability study was conducted.

The final usability study consisted of two rounds of usability study to examine the enhanced Microsoft Word Add-In prototype. Additional participants who are blind were recruited to participate in the first and second rounds of the final usability study. Once the first round of usability study was completed the Microsoft Word Add-In prototype was enhanced based on the participants' comments and suggested improvements, and a second round of usability study was completed.

The next sections present the details of the final usability study, including the selection criteria for the participants, the environment configuration, the usability study procedure and tasks, the data collection methods and the results. The final implications and conclusion are presented in Chapter 11.

\subsubsection{Participant(s)}

Eleven distinct participants were recruited for the usability study from the Members of the National Federation of the Blind and the Maryland State Library for the Blind and Physically Handicapped (MSLBPH). The participants were blind, with no residual vision, had at least 6 to 10 years computer experience, at least 1 to 5 years' experience with Microsoft Word, and at least 6 to 10 years' experience with the JAWS Screen Reader. Two rounds of usability study were conducted with 5 participants in the first round and 6 participants in the second round. None of the participants participated in 
both usability study sessions. The specific selection criteria for participants who are blind can be found in section "9.2.1.2 Participants and Study Location."

\subsubsection{Environment Configurations}

The usability study was conducted using a Dell Latitude E6400 Laptop Computer running Windows 7 Enterprise. The installed applications consisted of Microsoft Word 2010, JAWS version 13, and the developed Microsoft Word Add-In prototype. The external hardware consisted of a standard keyboard.

The JAWS Screen Reader speed setting ranges from 40 (slow) to 150 (fast). For this study the JAWS Screen Reader was set to "Eloquence" at the default speed setting of 57 , which is a normal speech pace. The default speed setting was used for all participants of this study. Adjustments were not made to increase or decrease the voice speed.

\subsubsection{Procedure}

The same procedure utilized during the pilot study was adopted for this round of the study. 
10.6.4 Data Analysis and Results

10.6.4.1 Demographics

Demographical data was collected from the responses collected from the prestudy questionnaire. Table 34 presents the demographical and experience data collected from the participants of the two rounds of usability study. 
Table 34. Demographical Data

\begin{tabular}{|c|c|c|}
\hline Demographical Data & $1^{\text {st }}$ Round & $2^{\mathrm{ND}}$ Round \\
\hline \multicolumn{3}{|c|}{ Gender } \\
\hline Male & $60 \%(3)$ & $16.7 \%(1)$ \\
\hline Female & $40 \%(2)$ & $83.3 \%(5)$ \\
\hline \multicolumn{3}{|c|}{ Age Range } \\
\hline Age 19 to 29 & $20 \%(1)$ & $16.7 \%(1)$ \\
\hline Age 30 to 39 & $0 \%(0)$ & $16.7 \%(1)$ \\
\hline Age 40 to 49 & $40 \%(2)$ & $16.6 \%(1)$ \\
\hline Age 50 to 59 & $20 \%(1)$ & $50 \%(3)$ \\
\hline Age 60 and Over & $20 \%(1)$ & $0 \%(0)$ \\
\hline \multicolumn{3}{|c|}{ Computer Experience } \\
\hline $\begin{array}{l}6 \text { to } 10 \text { years of computer } \\
\text { experience }\end{array}$ & $20 \%(1)$ & $0 \%(0)$ \\
\hline $\begin{array}{l}11 \text { to } 20 \text { years of } \\
\text { computer experience }\end{array}$ & $40 \%(2)$ & $16.7 \%(1)$ \\
\hline $\begin{array}{l}21 \text { to } 30 \text { years of } \\
\text { computer experience }\end{array}$ & $40 \%(2)$ & $66.6 \%(4)$ \\
\hline Over 30 years & $0 \%(0)$ & $16.7 \%(1)$ \\
\hline \multicolumn{3}{|c|}{ Primary Use of the Computer } \\
\hline Computer for work & $40 \%(2)$ & $83.3 \%(5)$ \\
\hline $\begin{array}{l}\text { Computer for school / } \\
\text { college }\end{array}$ & $0 \%(0)$ & $16.7 \%(1)$ \\
\hline $\begin{array}{l}\text { Computer for } \\
\text { entertainment }\end{array}$ & $20 \%(1)$ & $0 \%(0)$ \\
\hline Other & $40 \%(2)$ & $0 \%(0)$ \\
\hline \multicolumn{3}{|c|}{ JAWS Experience Level } \\
\hline 1 to 5 years & $0 \%(0)$ & $0 \%(0)$ \\
\hline 6 to 10 years & $20 \%(1)$ & $16.7 \%(1)$ \\
\hline 11 to 20 years & $80 \%(4)$ & $16.7 \%(1)$ \\
\hline 21 to 30 years & $0 \%(0)$ & $66.6 \%(4)$ \\
\hline
\end{tabular}




\begin{tabular}{|c|c|c|}
\hline Demographical Data & $1^{\text {st }}$ Round & $2^{\mathrm{ND}}$ Round \\
\hline \multicolumn{3}{|c|}{ Microsoft Word Experience Level } \\
\hline 1 to 5 years & $20 \%(1)$ & $0 \%(0)$ \\
\hline 6 to 10 years & $40 \%(2)$ & $20 \%(1)$ \\
\hline 11 to 20 years & $40 \%(2)$ & $80 \%(4)$ \\
\hline \multicolumn{3}{|c|}{ Microsoft Word Use Frequency } \\
\hline Daily & $60 \%(3)$ & $80 \%(4)$ \\
\hline Several times a week & $40 \%(2)$ & $20 \%(1)$ \\
\hline
\end{tabular}

Most participants were in the age range of 50 to 59 years old (4 participants) and the age range of 40 to 49 years old ( 3 participants). Two participants were 19 to 29 years old and one participant was in each of the age ranges of 30 to 39 years old and 60 years old and older. Most participants (6) had 21 to 30 years computer experience and three participants had 11 to 20 years computer experience. One participant had 6 to 10 years computer experience and one participant had over 30 years computer experience. All of the participants (11) utilized the computer daily, and the majority of the participants (7) utilized the computer at work. The majority of the participants (5) had 11 to 20 years' experience and four participants had 21 to 30 years' experience utilizing the JAWS Screen Reader. Most of the participants (10) had at least one years' experience utilizing Microsoft Word. One participant had less than one years' experience utilizing Microsoft Word. The majority of the participants (7) utilized Microsoft Word daily. The broad age ranges and experience levels of the participants of the usability study provide a good mix of age and computer and screen reader experience. 


\subsubsection{Prior Experiences with the Microsoft Word Interface}

The participants reported candidly about their prior experiences with the Microsoft Word interface in regard to track changes. They reported having issues with the ribbon menu, finding track changes and comments, and reading issues with the JAWS screen reader. Often, persons who are blind attempt to utilize track change and comments features of Microsoft Word, but encounter difficulties. One participant commented, "I have tried to use track changes from time-to-time. I had problems selecting and finding items." Another participant commented, "It is difficult to find comments and determine what changes others have made." Since, persons who are blind cannot find the revisions and comments; they cannot participate in collaborative writing independently. In addition to the inability of finding track changes and comments, the participants commented on their inability to properly interact with the Microsoft Word Ribbon Menu. The participants commented, "I never understood the track change and the ribbon is difficult but manageable," and "Some of the menu options do not read properly with JAWS." Given the difficulties with track changes and the access and use of the ribbon menu, the Microsoft Word Add-In prototype evolved to bypass the ribbon menu and to present track changes and comments so the JAWS Screen Reader could properly read the revisions and comments. 


\subsubsection{Performance}

The usability study participants completed all (100\%) tasks utilizing the Microsoft Word Add-In, besting the sixty-two percent (62\%) task completion rate of the participants of the baseline usability study utilizing the standard Microsoft Word interface. This finding provided some evidence that the Microsoft Word Add-In prototype improves participant performance while completing tasks associated with track change and comment features of Microsoft Word.

Figure 27 presents the task completion mean in terms of seconds for the two rounds of the usability study and the Baseline usability study.

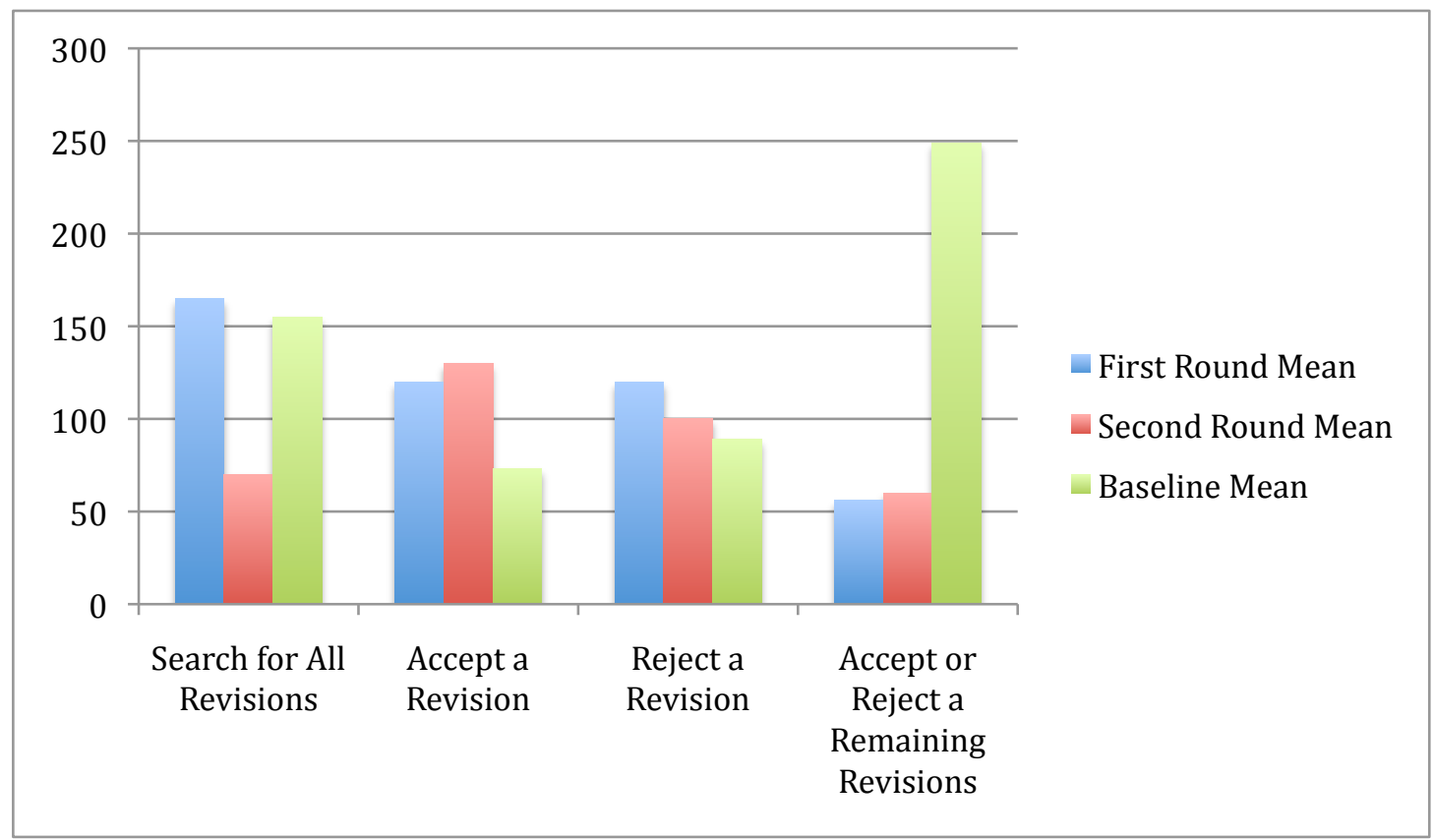

Figure 27 Microsoft Word Add-In Prototype - Usability Study - Task Completion Mean 
Comparing to the Baseline usability study results to the Microsoft Word Add-In prototype usability study results, improvements were identified for the second-round in the, "Search for all revisions and comments" and "Accept or reject a remaining revision or comment" tasks (Figure 27). Whereas, comparing to the Baseline usability study results to the Microsoft Word Add-In prototype usability study results, improvements were not identified in the, "Accept or reject a revision or comment" tasks (Figure 27). During the Baseline usability study (Schoeberlein \& Wang, 2012) only 38\% of the participants completed these tasks versus $100 \%$ completion rate for the Microsoft Word Add-In prototype usability study for the tasks being compared.

The participants of second round of the usability study largely improved on the mean time to complete the search for all revisions task (Figure 27). The first round participants took an average of 165 seconds to complete the search for all revisions task; whereas, the second round participants took an average of 70 seconds to complete the search for all revisions task. The participants from both rounds had similar mean completion times in regard to accepting a revision (120 seconds versus 130 seconds), rejecting a revision (120 seconds versus 100 seconds), and accept/reject all remaining revisions (56.25 seconds versus 60 seconds).

The participants of second round of the usability study improved on the standard deviation time to complete the search for all revisions task. The first round participants had a standard deviation of 57.44 seconds to complete the search for all revisions task; whereas, the second round participants had a standard deviation of 24.40 seconds to complete the search for all revisions task. The first round participants had a standard deviation of 0 seconds to accept a revision task; whereas, the second round participants 
had a standard deviation of 70.14 seconds to accept a revision task. The participants of both had similar standard deviation completion times in regard to rejecting a revision (48.98 seconds versus 48.98 seconds), and accept/reject all remaining revisions (7.5 seconds versus 0 seconds).

The improvements of completion times of the second-round participants over the first-round participants can be attributed to two factors. The first factor for the improved completion times was the improved prototype interface, which directly allowed for the acceptance and rejection of the revisions. The participants of the second-round no longer utilized the Microsoft Word Ribbon Menu to accept or reject revisions, providing the participants of the second-round a smoother process for searching, accepting and rejecting revisions. The second factor for the improved completion times was the increased Microsoft Word experience level of the participants of the second-round. Eighty percent of the second-round participants had 11 to 20 years' experience utilizing Microsoft Word; whereas, only twenty percent of the first-round participants had 11 to 20 years' experience utilizing Microsoft Word (Table 34).

\subsubsection{Analysis of the audio/video recordings}

For the analysis of the audio/video recording, a transcript was developed and coded from the usability studies. Two independent coders examined and coded the transcript in order to validate the categories identified from the annotated list collected while reviewing the audio/video transcripts. Twenty-four cases, belonging to three categories, were identified with a Cohen's Kappa of 100\%, providing evidence of acceptable agreement and reliability between the coders. The categories identified were usability, improvement request and issues (Table 35). 
Table 35. Category and Element Examples ( $1^{\text {st }}$ round $)$

\begin{tabular}{|c|c|}
\hline Category & Element Examples \\
\hline $\begin{array}{l}\text { 1. Usability } \\
\text { features }\end{array}$ & $\begin{array}{l}\text { Right mouse click to access the Microsoft Word Add-In } \\
\text { Command Bar } \\
\text { The paragraph is presented - enter to continue, escape to } \\
\text { exit } \\
\text { - Revision is presented - enter to continue, escape to select } \\
\text { the revision }\end{array}$ \\
\hline $\begin{array}{l}\text { 2. Improvement } \\
\text { request }\end{array}$ & "How can I repeat the revision?" \\
\hline 3. Issues & $\begin{array}{l}\text { There are too many steps to accept a revision - Enter "Alt- } \\
\text { r" to select the "Review" ribbon menu tab, enter "A" for } \\
\text { the "Accept" drop-down menu, enter down-arrow to } \\
\text { "Accept" the revision } \\
\text { - There are too many steps to reject a revision - Enter "Alt-r" } \\
\text { to select the "Review" ribbon menu tab, enter "J" for the } \\
\text { "Reject" drop-down menu, enter down-arrow to "reject" } \\
\text { the revision }\end{array}$ \\
\hline
\end{tabular}




\subsubsection{Microsoft Word Add-In Prototype $-1^{\text {st }}$ Round Enhancements}

The "original" logic flow of the prototype moved from the Microsoft Word AddIn Command Bar to the Microsoft Word Ribbon Menu in order to accept/reject a track change. This movement was cumbersome for the participants to use. The logic flow followed these steps:

1. Present the sentence or paragraph for the revision or comment;

2. Present the revision or comment;

3. Set the focus to the revision or comment;

4. The participant presses the "Alt-r" keys to select the "Review" ribbon menu tab;

5. The participant presses the "A" key for the "Accept" drop-down menu, or presses the "J" key for the "Reject" drop-down menu;

6. The participant presses the down-arrow to select the sub-menu item to "Accept" or "Reject" the revision.

Based on the suggested improvements (Table 35) from the participants of the $1^{\text {st }}$ round of usability study, enhancements were made to the Microsoft Word Add-In prototype interface. The enhancements included avoiding the Microsoft Word Ribbon Menu, reducing the number of steps necessary to accept or reject a track change, providing command buttons for "Yes/No" responses, and repeating the text of the paragraph or sentence when requested. 
The result of the first round improvements consisted of the following steps:

1. Right mouse click to access the Microsoft Word Add-In Command Bar;

2. Present the Sentence or paragraph (Figure 28);

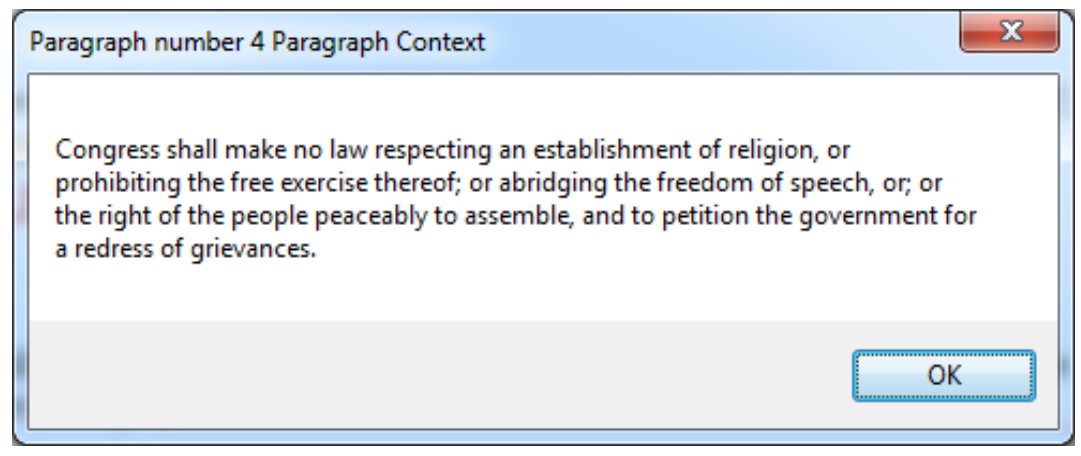

Figure 28 Paragraph Context

3. Present the revision and revision type (Figure 29);

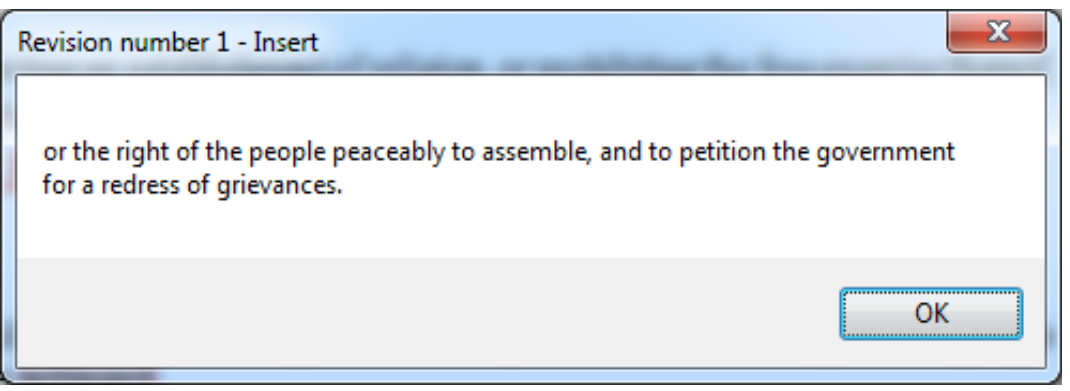

Figure 29 Insert Revisions

4. Ask, "Do you want to accept the revision? Yes/No" (Figure 30);

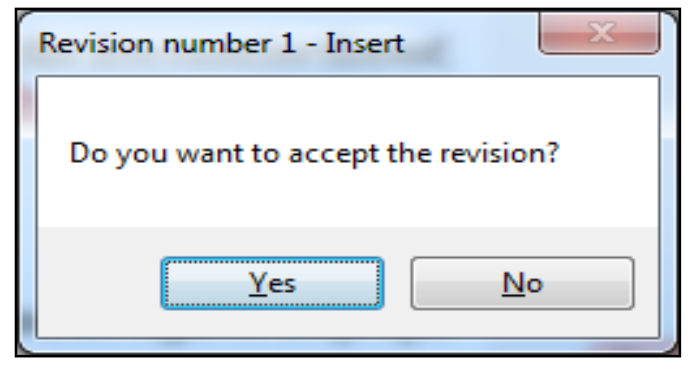

Figure 30 Accept a Revision 
After the user selected "No" to the question "Do you want to accept the revision?

5. Ask, "Do you want to reject the revision? Yes/No" (Figure 31); and,

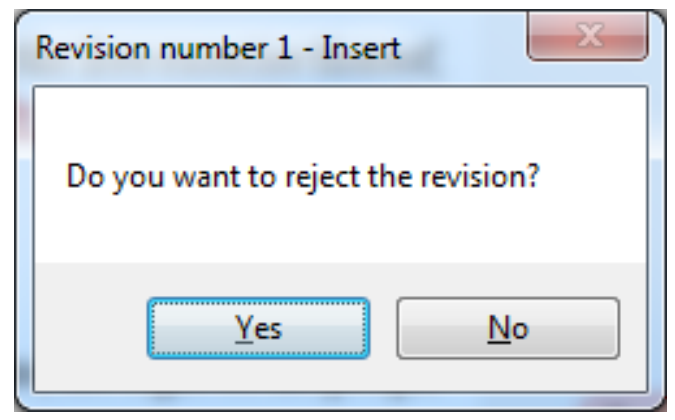

Figure 31 Reject a Revision

After, the user selected "No" to both previous questions.

6. Ask, "Do you want to repeat the revision? Yes/No" (Figure 32).

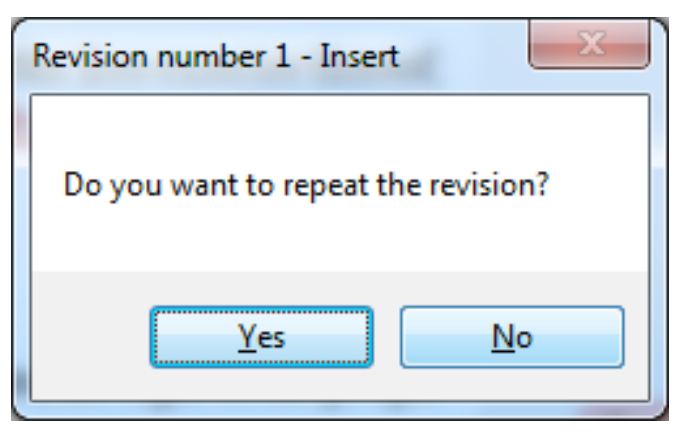

Figure 32 Repeat a Revision

Once a selection is made, the logic cycle is exited.

The "new" logic flow reduces the steps necessary to accept or reject a change and avoids the Microsoft Word Ribbon Menu entirely. Questions for accepting or rejecting the track change were added, making the acceptance and rejection process easier. Prior to this change, the participants had to manually access the Microsoft Word Ribbon Menu in order to accept or reject a revision. Now, the acceptance and rejection are programmed into the Microsoft Word Add-In prototype. The prototype's command buttons are labeled 
with "Yes" and "No" for responses to all questions. So, prior knowledge is unnecessary to navigate the prototype. To repeat a revision, the logic cycle begins again on the same revision. This feature is basically utilized to listen to the revision again, in order to double-check anything missed during the original reading of the revision. Overall, these improvements streamlined the logic flow of the interface and reduced the number of steps necessary to accept or reject a revision or comment. 


\subsubsection{Perception of Collaborative Writing Add-In Prototype}

The data in Figure 33 provides the percentage of agreement and the percentage of disagreement with each question or statement of the post-study questionnaire from the two rounds of the usability study. The questions present the opinions of the participants' in regard to their experiences of utilizing the Microsoft Word Add-In prototype.

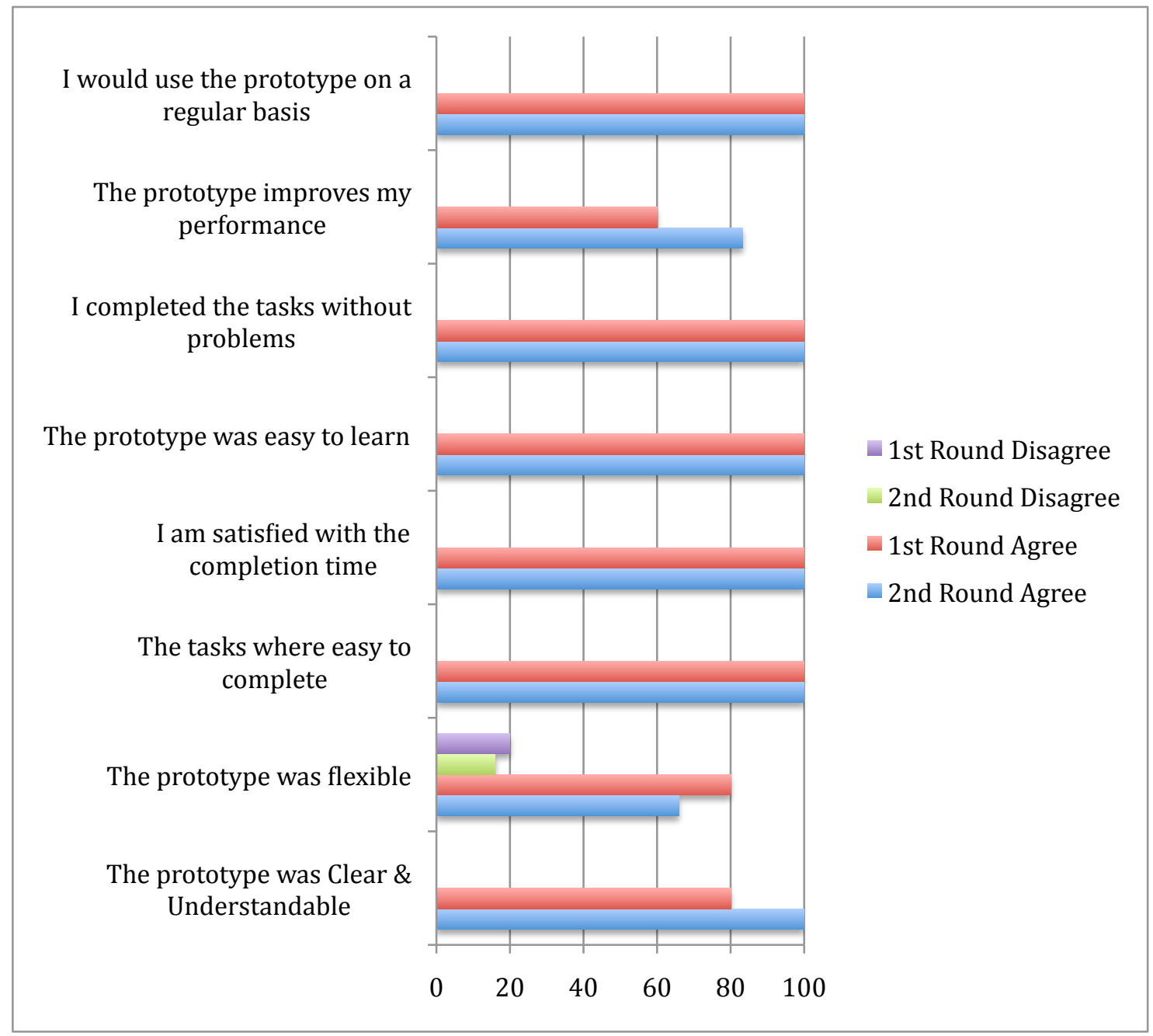

Figure 33 Analysis of Post-Study Questionnaire ( $1^{\text {st }}$ and $2^{\text {nd }}$ rounds) 
All of the participants of the first and second-round agreed that they would use the Microsoft Word Add-In prototype on a regular basis, that the prototype was easy to learn, that they were satisfied with their tasks' completion times, that they completed the tasks without any problems, and that the tasks were easy to complete using the prototype.

When the participants were asked if they felt the prototype improved their performance, $60 \%$ of the first-round participants agreed, while $83.3 \%$ of the secondround participants agreed. Eighty percent of the first-round participants and $100 \%$ of the second-round participants were in agreement that the prototype was clear and understandable. The enhancement to the Microsoft Word Add-In prototype between the two rounds, which removed the use of the Microsoft Word Ribbon Menu to search for revisions and comments, was the main reason for the improvement of favorable responses. The removal of the need to access the MS Word Ribbon Menu streamlined the process of searching and retrieving revisions and comments. Additionally, the Microsoft Word experience level between the second-round participants versus the first-round participants (Table 34) may have played a part in the improvement of favorable responses.

The only negative responses were in regard to the lack of flexibility of the prototype. When the participants were asked their opinions in regard to the flexibility of the prototype, $20 \%$ of the first-round participants and $16 \%$ of the second-round participants responded that the prototype was not flexible. These responses were not entirely surprising. The prototype was designed to present the paragraph or sentence containing a revision or comment, followed by the actual revision or comment, and finished with the asking the participant to accept, reject or repeat the revision or 
comment. This procedural control could be seen as not being very flexible. However, the “Revisions Bookmarks" and "Comments Bookmarks" options (Figure 36) provide direct access to the revisions or comments with less procedural control.

Overall the Microsoft Word Add-In prototype was accessible and usable for revisions and comments features of Microsoft Word, and the participants liked the prototype interface. 
10.6.4.7 More Feedback Collected on Collaborative Writing Experiences

Feedback on the collaborative writing experience, after using the Microsoft Word Add-In, were also collected from the responses to open-ended questions of the post-study questionnaire (Table 36 and 37). The focus of these responses was the Microsoft Word Add-In prototype interface.

Table 36. Perceptions of the Microsoft Word Add-In Prototype (1st round)

\begin{tabular}{|c|c|}
\hline Category & Sample Comments \\
\hline Positive & $\begin{array}{l}\text { - I like the fact that it allows you to choose to keep the track } \\
\text { changes or to reject them. } \\
\text { - The commands are easy and simple to understand. } \\
\text { - I discovered it was actually much easier than I ever } \\
\text { imagined. As we walked through, I actually discovered } \\
\text { how nicely the process flowed from one step to the next. } \\
\text { - I had no trouble understanding this at all and found it very } \\
\text { easy to perform the duties asked of me in the end. } \\
\text { The revision or comment text appears much easier to } \\
\text { distinguish from existing text than when using the word } \\
\text { features themselves. } \\
\text { Once the user has proper orientation, this is an easy and } \\
\text { helpful tool. }\end{array}$ \\
\hline $\begin{array}{l}\text { Suggested } \\
\text { Improvements }\end{array}$ & 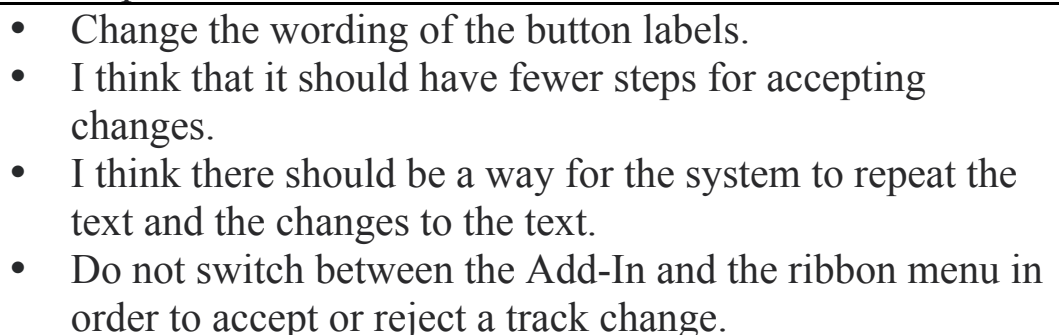 \\
\hline
\end{tabular}


Table 37. Perceptions of the Microsoft Word Add-In Prototype (2nd round)

\begin{tabular}{|l|ll|}
\hline \multicolumn{1}{|c|}{ Category } & \multicolumn{1}{c|}{ Sample Comments } \\
\hline Positive & $\begin{array}{l}\text { It does identify comments and track changes. } \\
\text { - }\end{array}$ & $\begin{array}{l}\text { The dialogs follow the Windows usual dialogs. } \\
\text { Much easier to locate track changes and make } \\
\text { corrections if need be. Also, it makes me feel even } \\
\text { more independent when it comes to doing my work } \\
\text { without calling on a sighted person to do the track } \\
\text { changes for me. }\end{array}$ \\
& $\begin{array}{l}\text { It was simple to use it once. I understood how it } \\
\text { worked, but the best part was that it only took a few } \\
\text { minutes to fully understand how to use this program. }\end{array}$ \\
\hline Suggested & $\begin{array}{l}\text { Some help either in the form of a help file or context } \\
\text { Improvements }\end{array}$ & $\begin{array}{l}\text { sensitive help. } \\
\text { Allow an escape to interrupt the process and have that } \\
\text { escape land your cursor in the text last read. } \\
\text { Create a hot key to bring up the paragraph and } \\
\text { sentence review choices. }\end{array}$ \\
\hline
\end{tabular}

10.6.4.8 Microsoft Word Add-In Prototype - $2^{\text {nd }}$ Round Enhancements.

Based on the suggested improvements from the participants of the $2^{\text {nd }}$ round of usability study (Table 37), adjustments were made to the Microsoft Word Add-In prototype interface. The adjustments included the following: Inclusion of a "Help" Menu (Figure 36); the ability to continue or exit (Figure 37); and, hot keys to access the command bar options (Figure 38). These features were developed, but not usability tested and could be tested in future usability studies. 


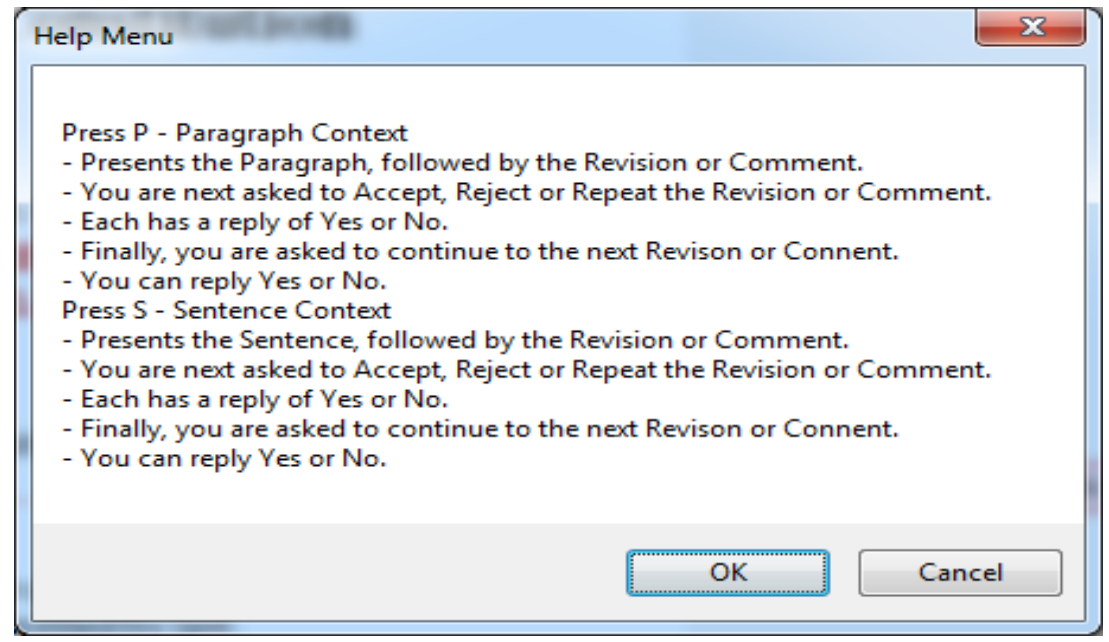

Figure 34 Help Menu

Pressing the letter ' $\mathrm{H}$ ' or ' $h$ ' on the Microsoft Word Command Bar accesses the help Menu (Figure 34). The Help Menu provides a description of the key press to access a menu option on the Microsoft Word Command Bar and a brief description of the option's functionality.

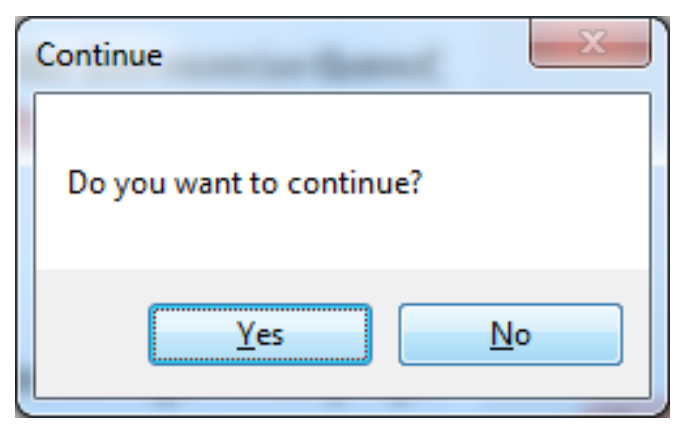

Figure 35 Continue or Exit

The "Continue" Message Box (Figure 35) was added to the logic cycle, to enable the end user the ability to continue or exit the examination of revisions and comments. If the end user chooses to continue, the next comment or revision will be presented. Otherwise, the user will be lead back to the document. 


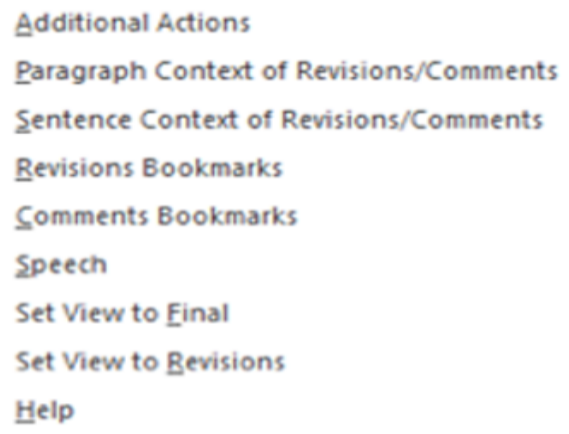

Figure 36 Microsoft Word Command Bar Hot Key Accesses

Hot key values are displayed on the Microsoft Word Command Bar (Figure 36), and identified by the underscored character. The JAWS screen reader emphasizes the underscore value when reading the menu option. Pressing the key that is underscored enables access to the menu item. 


\section{CONCLUSION}

\subsection{Introduction}

Collaborative writing research is necessary in order to understand the needs of persons who are blind and to provide collaborative interfaces that are easy to access and use. This research is significant in that it focuses on the importance of presenting the context of a revision or comment in order to improve understanding. This improved understanding enables persons who are blind make intelligent decisions when determining whether to accept or to reject a revision. Therefore, presenting the context of a revision or comment is critical!

The developed prototype provided evidence that presenting messages and questions for guiding collaboration enhanced the abilities of persons who are blind to independently participate in collaborative writing (Schoeberlein \& Wang, 2013). No longer do persons who are blind require the assistance of visually able persons to aid them in understanding and editing collaborative documents (Schoeberlein \& Wang, 2013). Now, with the availability of the context of either a sentence or a paragraph, and messages for presenting the revisions and the comments, persons who are blind are independently participating in collaborative writing (Schoeberlein \& Wang, 2013). The next sections present the limitations, results, implications, conclusion and future research directions. 


\subsection{The Research Plan}

The research plan consisted of several preliminary steps, which led to the identification of the research focus for this dissertation. Once the focus on collaborative writing was identified, the research questions to be investigated were developed. In order to answer the two research questions, a five-step research design was developed.

\subsubsection{Preliminary Research Steps}

The research plan consisted of several preliminary steps in order to understand how persons who are blind worked collaboratively, and the accessibility and usability issues encountered while they engaging in collaborative work. To establish an understanding how persons who are blind worked collaboratively and the accessibility and usability issues they encountered, a literature review (Schoeberlein \& Wang, 2009a) on accessibility, usability and collaborative technology was conducted. Accessibility evaluations of web-based and Windows-based collaborative applications (Schoeberlein \& Wang, 2009b) provided a list of accessibility issues of select collaborative applications. Focus groups (Schoeberlein \& Wang, 2011) were conducted to observe first-hand both accessibility and usability issues encountered while collaborating. At the conclusion of the literature review (Schoeberlein \& Wang, 2009a), accessibility evaluations (Schoeberlein \& Wang, 2009b) and the focus group studies (Schoeberlein \& Wang, 2011) a summary of accessibility and usability issues related to collaborative technologies were identified for collaborative writing, email, Chat and group scheduling applications. 


\subsubsection{Collaborative Writing and the Research Questions}

The result of the preliminary research steps included a set of research opportunities on the accessibility and usability issues as they related to collaborative technologies (Chapter 8). Collaborative writing was selected as the focus, since it was reported that track changes were difficult to use and understand (Schoeberlein \& Wang, 2011). In order to improve collaborative writing, two research questions were developed as a roadmap to help identify the procedures and methodologies of this research. The research questions investigated were:

- RQ1: What accessibility and usability issues do collaborative writing technologies, such as Microsoft Word and Google Docs, present for persons who are blind?

- RQ2: How should a collaborative writing interface is designed in order to be accessible and usable for persons who are blind?

In order to answer these two questions, a research design was developed to identify the steps necessary to answer the research questions and to identify when the research was complete.

\subsubsection{The Baseline, Pilot and Additional Usability Studies}

The research design consisted of five distinct steps (Chapter 9) which included a baseline study of Microsoft Word and Google Docs (Schoeberlein \& Wang, 2012), the development of an accessible collaborative writing prototype for Microsoft Word (Schoeberlein \& Wang, 2013), a pilot usability study (Schoeberlein \& Wang, 2013) to examine the prototype and validate the study procedure and measurements, additional 
enhancements to the prototype, the study procedure and measurements (Chapter 10), and a final set of usability studies to examine and improve the prototype for accessible collaborative writing (Chapter 10).

\subsection{Limitations}

All phases of this research had some limitations, whether it was the quality of the available automated accessibility evaluation tools or the number and diversity of the available participants, which are reported in this section.

\subsubsection{Preliminary Research Limitations}

The preliminary research steps consisted of the web-based and Windows-based automated evaluations (Schoeberlein \& Wang, 2009b) and the focus groups (Schoeberlein \& Wang, 2011). The automated tools, Fujitsu's Web Accessibility Inspector (2008) for evaluating WCAG 1.0 compliance and Adaptive Technology Resource Center ATRC Web Accessibility Checker (2009) for evaluating WCAG 2.0 compliance, detected an extensive amount of false-positives during the web-based automated accessibility evaluations (Schoeberlein \& Wang, 2009b). Therefore, it is important to understand this limitation of the automated tools of detecting false-positives and to consider manual evaluations as a follow-up step to provide a more complete and accurate accessibility evaluation.

For the focus groups (Schoeberlein \& Wang, 2011), the main limitation of these studies was recruiting participants who are blind to participate in these studies. The National Federation of the Blind was a good source of potential participants. However, these participants seemed to be more capable users of technology such as computers, Microsoft Word, and screen readers. Having homogeneous participants in terms of skill 
levels does not afford you the diversity necessary to uncover some issues related to lack of experience. Recruiting participants who are blind at the local Colleges and Universities was difficult, since the Institutions would not allow direct contact with the Students and would simply broadcast an email message soliciting participation. As a rule, if monetary resources are available, they are to be discussed in private sessions and not to be publically broadcasted. Therefore, the possible Student participants who would normally participate if monetarily rewarded could not be solicited via email and did not participate.

\subsubsection{The Baseline, Pilot and Additional Usability Studies Limitations}

The research questions focused on two specific collaborative writing applications and should other collaborative writing applications been investigated, different results could exist. Microsoft Word and Google Docs provided two perspectives of synchronous and asynchronous collaborations. Unfortunately, Google Docs proved to be entirely inaccessible to the participants who are blind (Schoeberlein \& Wang, 2012). So, this research was limited to asynchronous collaborations only, even though the results of this research are applicable to both asynchronous and synchronous. The context of a paragraph or sentence and the use of messages to perform the accept/reject processes for revisions are applicable for both asynchronous and synchronous collaborations.

The usability studies were a major component of this research used to identify accessibility and usability issues and to validate the accessible collaborative writing prototype. The number of participants of the usability studies and their skill levels prevents making any significant statistical claims. Finding and recruiting participants who are blind is extremely difficult and time consuming, so a more practical approach may be to develop an online web-based solution to reach a larger target audience. 
Considering the available time limitation and the lack of resources for application and Web servers, this alternative proved to be unapproachable at the time it was considered.

The author of this dissertation developed the collaborative writing prototype, with the advice provided by a Faculty Advisor and the participants of this research. The Author's limited experience developing collaborative writing applications, which includes a Microsoft Word Add-In with a Microsoft Word Command Bar ("MSDN Microsoft Word Command Bar," 2013) limited the features provided. Although the features were very comprehensive, had additional experienced developers been included, better alternatives could have been developed. For the features of the prototype, refer to "10.3.2 Microsoft Word Add-In Features Proposed." Basically, additional developers and the synergies they produce were not available for this research, limiting this development. Even with these limitations, the resulting research findings and the developed prototype are both of value for further research.

\subsection{Results}

To answer the research questions, a baseline usability study using Microsoft Word and Google Docs (Schoeberlein \& Wang, 2012) was conducted to identify the access and use issues associated with collaborative writing, and a prototype was designed, developed and evaluated during a pilot usability study and two additional usability studies. The proposed Microsoft Word Add-In prototype (Word Add-Ins, 2013) provided many benefits for collaborative writing. Since the Microsoft Word Add-In is a component of Microsoft Word, it was seamlessly integrated into Microsoft Word. Users do not need any prior knowledge to utilize the Microsoft Word Command Bar ("MSDN Microsoft 
Word Command Bar," 2013), since they can simply right-click anywhere on a document to have direct access to the commands. With this direct access feature, there is no need to memorize complex key stoke combinations when working on the document. Therefore, the cognitive load was reduced to prevent overload. Since the Add-In was applied at the application level, it works with all Microsoft Word documents. It provides descriptive information regarding any comments/revisions including their sequence as well as type and content of the revisions. In addition, users are presented with an overview of the document and context of the revision/comments, so that users are aware of the context of any action. The Add-In can be upgraded to different versions of Microsoft Word and is compatible with the JAWS screen reader. This eliminates the need for additional investment for the application to be accessible.

This prototype was presented during a pilot usability study (Schoeberlein \& Wang, 2013) and two additional rounds of usability studies (Chapter 10) to participants who are blind, to determine if the interface design was accessible, usable and improved performance.

The resulting prototype was considered easy to learn, easy to use, clear and understandable, improved their performance, and would be utilized on a regular basis to do similar tasks (Schoeberlein \& Wang, 2013). Even though this prototype was specific to Microsoft Word, this interface design of utilizing a context sensitive menu and hot keys, and the importance of presenting the revisions' and comments' context along with the actual revisions and comments are transferrable to all collaborative writing tools and applications. This prototype's features are universal for accessible and usable collaborative writing. 


\subsection{Implications}

Adaptive technologies, accessibility guidelines, automated evaluations, field studies, focus group studies, the pilot usability study and the additional usability studies all have implications that may not seem obvious, and so this section is dedicated to presenting these implications.

\subsubsection{Preliminary Research}

Adaptive technologies and accessibility guidelines are essential for providing accessibility and usability support for persons with disabilities (Schoeberlein \& Wang, 2009a). Hence, it is important to have an understanding of both adaptive technologies and accessibility guidelines in order to design and implement accessible and usable collaborative applications.

Automated evaluations may be a necessary first step in evaluating an application for accessibility (Schoeberlein \& Wang, 2009a). However, additional manual validations of the Web site and Web pages are necessary. All too often, automated evaluations identify false-positives that must be further evaluated (Schoeberlein \& Wang, 2009b). Once the Web Site and Web pages are manually evaluated, accessibility changes to a Web Site and their Web pages can be implemented. Even though an automated tool utilized to evaluate accessibility identifies accessibility issues, the Web Site may function properly.

During the pilot focus group study, the participants were considered experienced users of collaborative technologies and the JAWS Screen Reader (Schoeberlein \& Wang, 2011a). It is important to select a more diverse group of participants in order to not bias 
the results. The experience level of the participants may have biased the results from the pilot focus group study (Schoeberlein \& Wang, 2011a). So, to correct this possible bias, additional focus group studies were conducted at the Mid-Atlantic State Convention of the National Federation of the Blind in Annapolis, Maryland (Schoeberlein \& Wang, 2011b).

Even though all of the participants of the pilot focus group study (Schoeberlein \& Wang, 2011a) were capable users of the operating system, the selected collaborative applications, and screen reader software, they still expressed the apprehension of utilizing new collaborative applications. The root cause of this apprehension was related to the steep learning curve and the inability to solve the accessibility issues encountered (Schoeberlein \& Wang, 2011a). Therefore, it is important that accessibility and usability evaluations be conducted with collaborative applications prior to their distribution to the marketplace. Additionally, the participants of the pilot focus group study expressed the need for consistency when collaborative applications and screen reader software are upgraded, so they do not lose prior features they are accustom to utilizing (Schoeberlein \& Wang, 2011a). As different versions of the screen reader software are developed, older versions of collaborative applications may no longer be accessible (Schoeberlein \& Wang, 2011a). To resolve this inconsistency between upgrades of collaborative applications and screen reader software, it is critical that these technologies be tested and evaluated together.

All of the findings from the pilot focus group study, the additional focus group studies and the field studies point to the need to improve the interaction between the screen reader and collaborative and other applications (Schoeberlein \& Wang, 2011a). 
What messages or content are presented, how much content is presented, and how understandable is the content are key to accessibility and usability. Additionally, handling random events is crucial to successful interactions between any application and a screen reader. Once again, we can see the importance of integrated testing and evaluations between the collaborative applications and screen reader software.

These additional focus group studies (Schoeberlein \& Wang, 2011b) further confirmed the findings received from the pilot focus group study (Schoeberlein \& Wang, 2011a). The participants of the additional focus group studies had more diverse backgrounds and they worked in entirely separate groups; thus, new information in the form of accessibility and usability issues and collaborative applications were identified (Schoeberlein \& Wang, 2011b). Collaborative applications, not previously identified, included Microsoft Outlook Express, and Google Calendar, which are utilized for email and group calendars, Novell GroupWise and Microsoft SharePoint were solid examples of groupware, and additional word processing applications including Microsoft SharePoint and Word Perfect (Schoeberlein \& Wang, 2011b). The tasks supported are email, text messaging, task tracking, group calendar, and collaborative writing (Schoeberlein \& Wang, 2011b). Therefore, these collaborative tasks can be seen as essential to collaboration and some form of collaborative application is required to support these tasks.

\subsubsection{The Baseline, Pilot and Additional Usability Studies}

The usability studies highlighted key areas where persons who are blind had difficulty completing certain collaborative writing tasks (Schoeberlein \& Wang, 2012, 2013). The participants who are blind considered revisions and comments difficult to 
distinguish and understand unless they accepted all of the revisions before reviewing the document. Additionally, the participants identified the context of the comments and revisions as being important to their understanding of the revisions and comments. Without the context, the participants could not determine whether to accept or reject the revisions and comments. Therefore, it is critical that when presenting revisions and comments for acceptance or rejection, that the context for the revisions and comments be presented too!

The Microsoft Command Bar ("MSDN Microsoft Word Command Bar," 2013) enabled easy access to the collaborative writing features associated with track changes, and could be accessed by simply right-clicking anywhere on the open document (Schoeberlein \& Wang, 2013). Alternative interface approaches to the Microsoft Ribbon Menu should be considered for improved access and use.

\subsection{Future Research Directions}

For the existing collaborative writing prototype, the next research step would be a longitudinal study. During the longitudinal study, the collaborative writing prototype would be provided to select Members of the National Federation of the Blind or any other participants who are blind, so they could evaluate the prototype. This emersion of the collaborative writing prototype into the field would provide researchers with valuable feedback from day-to-day operations. The results of the day-to-day operations could include a list of access and use issues and suggestions for improvement. A list of additional features could be proposed and developed, or the collaborative writing prototype could be deemed satisfactory as is or abandoned as a solution. Either way, the 
longitudinal study would be the next logical step in evaluating the collaborative writing prototype prior to Marketplace distribution.

Since this research did not evaluate synchronous collaborations, a synchronous collaborative writing application should be evaluated. The synchronous collaborative writing application will provide additional challenges related to the concurrent access and use. Handling collision detection, when two or more participants select or edit the same sentence or paragraph concurrently will be an interesting challenge to review and if necessary to resolve. Additionally, recruiting participants for synchronous collaborative writing research may be easier to accomplish, since the participants can access and use the collaborative writing application at their leisure and at their own locations. One caution, in regard to synchronous collaborative writing research, could be the financial resources required for application servers and Web servers for the deployment of the application, and the additional manpower resources needed. Additional manpower resources would include developers and analysts for deployment and maintenance of the servers and the collaborative writing application. Researchers and analysts would be needed to collect and review the results from surveys and interviews of the participants.

\subsection{Conclusion}

This research consisted of a preliminary phase, a research topic and questions development phase and a prototype design development and evaluation phase.

All phases of this research project had identified limitations. The limitations consisted of too few diverse participants, false-positives when conduction automated accessibility evaluations on Web applications, accessibility issues of Google Docs preventing the detailed examination of a synchronous collaborative writing application, 
the Author's limited collaborative writing design and code development experience, the lack of additional experienced analysts and developers, and financial and manpower resources for implementing a Web-based solution.

Even with all these limitations, the collaborative writing prototype proved of value to the participants of this research (Schoeberlein \& Wang, 2013). Since the Microsoft Word Add-In ("Word Add-Ins," 2013) is a component of Microsoft Word; it was seamlessly integrated into Microsoft Word. Users do not need any prior knowledge to utilize the Microsoft Word Command Bar ("MSDN Microsoft Word Command Bar," 2013), since they can simply right-click anywhere on a document to have direct access to the commands (Schoeberlein \& Wang, 2013). Alternative interface approaches to the Microsoft Ribbon Menu, such as a Microsoft Word Command Bar (2013), should be considered for improved access and use.

The developed prototype provided evidence that presenting messages and questions for guiding collaboration enhanced the abilities of persons who are blind to independently participate in collaborative writing (Schoeberlein \& Wang, 2013). No longer do persons who are blind require the assistance of visual persons to aid them in understanding and editing collaborative documents (Schoeberlein \& Wang, 2013). Now, with the availability of the context of either a sentence or a paragraph, and messages for presenting the revisions and the comments, persons who are blind are independently participating in collaboratively writing (Schoeberlein \& Wang, 2013).

The resulting prototype was considered easy to learn, easy to use, clear and understandable, improved their performance, and would be utilized on a regular basis to do similar tasks (Schoeberlein \& Wang, 2013). Even though this prototype was specific 
to Microsoft Word, this interface design of utilizing a context sensitive menu and hot keys, and the importance of presenting the revisions' and comments' context along with the actual revisions and comments are transferrable to all collaborative writing tools and applications. This prototype's features are universal for accessible and usable collaborative writing. 


\section{APPENDIX A \\ Focus Group Study Questionnaire \\ Demographics}

1. Gender:

1 - Male

2 - Female

2. Age range:

$$
\begin{aligned}
& 1-18 \\
& 2-19-29 \\
& 3-30-39 \\
& 4-40-49 \\
& 5-50-5 \\
& 6-60 \text { and over }
\end{aligned}
$$

\section{Computer Experience}

1. Approximately how many years have you been using a desktop or laptop computer?

$$
\begin{aligned}
& 1 \text { - Less than } 1 \\
& 2-1 \text { to } 5 \\
& 3-6 \text { to } 10 \\
& 4-11 \text { to } 20 \\
& 5-21 \text { to } 30 \\
& 6-\text { Over } 30
\end{aligned}
$$

2. How frequently do you use a desktop or laptop computer?

1 - Daily

2 - Several times a week

3 - Weekly

4 - Several times a month

5 - Monthly 
3. Did you have any previous knowledge of any screen reader software?

$$
\begin{aligned}
& \text { 1-Yes } \\
& 2 \text { - No }
\end{aligned}
$$

If your answer to question number 3 is yes, which screen reader do you use?

If your answer to question number 3 is yes, approximately how many years of experience do you have using a screen reader?

$$
\begin{aligned}
& 1-\text { Less than } 1 \\
& 2-1 \text { to } 5 \\
& 3-6 \text { to } 10 \\
& 4-11 \text { to } 20 \\
& 5-21 \text { to } 30 \\
& 6-\text { Over } 30
\end{aligned}
$$

4. When you use the computer, what is the primary use?

$$
\begin{aligned}
& 1 \text { - Work } \\
& 2 \text { - Study } \\
& 3 \text { - Entertainment } \\
& 4 \text { - Other }
\end{aligned}
$$

\section{Identified Collaborative Application Experience}

1. Approximately how long have you worked with the identified collaborative application?

$$
\begin{aligned}
& 1 \text { - Less than } 1 \text { year } \\
& 2 \text { - } 1 \text { to } 5 \text { years } \\
& 3 \text { - } 6 \text { to } 10 \text { years } \\
& 4-11 \text { to } 20 \text { years } \\
& 5 \text { - } 21 \text { to } 30 \text { years } \\
& 6 \text { - Over } 30 \text { years }
\end{aligned}
$$

2. How frequently do you use the identified collaborative application?

$$
\begin{aligned}
& \text { - Daily } \\
& 2 \text { - Several times a week } \\
& \text { 3-Weekly } \\
& \text { - Several times a month } \\
& 5 \text { - Monthly }
\end{aligned}
$$


3. What do you use the identified collaborative application for?

4. Do you have any previous experience using the identified collaborative application and a screen reader like JAWS or Window's Eyes?

$$
\begin{aligned}
& 1 \text { - Yes } \\
& 2 \text { - No }
\end{aligned}
$$

5. If Yes to the previous question, approximately how many years' experience with the identified collaborative application and using a screen reader like JAWS or Window's Eyes?

$$
\begin{aligned}
& 1 \text { - Less than } 1 \\
& 2-1 \text { to } 5 \\
& 3-6 \text { to } 10 \\
& 4-11 \text { to } 20 \\
& 5-21 \text { to } 30 \\
& 6-\text { Over } 30
\end{aligned}
$$

6. Can you identify any difficulty you have encountered, if any, when interacting with the identified collaborative application

Please take notes during the study when you feel frustrated. 


\section{APPENDIX B}

List of Tasks / Identify Collaborative Applications / List of Known Accessibility Issues

1. List the tasks necessary to complete a group project. What steps does your Team take to complete a group project (such as brainstorming task)?

2. Prioritize the list of the tasks necessary to complete a group project. What is the priority order of the tasks for your Team to complete a group project?

3. Frequency of the list of tasks necessary to complete a group project. What is the frequency of each task in the list of tasks necessary for your Team take to complete a group project?

4. Do you use any software application for this kind of task? If you do, what kind of applications do you normally use? Which features of the application do you use normally?

5. Have you used any other software when working in groups? What are they? What features do you use the most frequently?

6. Have you ever experienced any difficulty when trying to access the software you discussed earlier? What kind of difficulty/problems have you ever experienced?

7. Can you rank order the list of issues identified earlier in terms of severity of the problem?

8. Are there any problems that you have experienced that stopped you from using the software?

9. How important is it to solve the problems identified earlier? Can you rate each problem in the scale of the following:

a. Urgent - the problem has to be solved. Otherwise, I cannot access the software.

b. Important - the problem should be solved, but I can still work around the issue.

c. Unimportant - the problem does not affect my day-to-day interaction, so I see no need to correct the problem.

10. How does your Team integrate the identified collaborative application into the group project? 
11. List the accessibility issues identified when using the identified collaborative application when completing a group project. What accessibility issues has your Team found when interacting with the identified collaborative application?

12. What types of support does your Team need to improve group collaboration?

13. What design improvements would you suggest for the interface of the identified collaborative application? 


\author{
APPENDIX C \\ Posttest Survey - Microsoft Word or Google Docs \\ (Usability study) \\ General observations
}

1. Answer all questions based on the collaborative writing tool you utilized during the usability study. Which collaborative writing tool did you utilized during the usability study?

1 - MS Word

2 - Google Docs

2. I feel this task was easy to complete?

1 - Strongly Agree

2 - Agree

3 - Neutral

4 - Disagree

5 - Strongly Disagree

6 - Does not apply

\title{
Computer Experience
}

3. My years of experience using a desktop or laptop computer helped me complete the task.

1 - Strongly Agree

2 - Agree

3 - Neutral

4 - Disagree

5 - Strongly Disagree

6 - Does not apply 


\section{Microsoft Word or Google Docs Experience}

1. My experience in working with Microsoft Word or Google Docs helped me complete the task.

$$
\begin{aligned}
& 1 \text { - Strongly Agree } \\
& 2 \text { - Agree } \\
& 3 \text { - Neutral } \\
& 4 \text { - Disagree } \\
& 5 \text { - Strongly Disagree } \\
& 6 \text { - Does not apply }
\end{aligned}
$$




\section{JAWS Screen Reader Experience}

1. My previous knowledge of the JAWS screen reader helped me complete the task.

$$
\begin{aligned}
& 1 \text { - Strongly Agree } \\
& 2 \text { - Agree } \\
& 3 \text { - Neutral } \\
& 4 \text { - Disagree } \\
& 5 \text { - Strongly Disagree } \\
& 6 \text { - Does not apply }
\end{aligned}
$$

\section{Opinions}

1. I am satisfied with the time I took to complete the task using Microsoft Word or Google Docs.

$$
\begin{aligned}
& 1 \text { - Strongly Agree } \\
& 2 \text { - Agree } \\
& 3 \text { - Neutral } \\
& 4 \text { - Disagree } \\
& 5 \text { - Strongly Disagree } \\
& 6 \text { - Does not apply }
\end{aligned}
$$

2. I am satisfied with the result of my work.

1 - Strongly Agree

2 - Agree

3 - Neutral

4 - Disagree

5 - Strongly Disagree

6 - Does not apply

3. I was able to finish my task using Microsoft Word or Google Docs without any problem.

1 - Strongly Agree

2 - Agree

3 - Neutral

4 - Disagree

5 - Strongly Disagree

6 - Does not apply 
4. Assuming I have access to Microsoft Word or Google Docs, I would use it on a regular basis in the future when $I$ am doing similar tasks.

1 - Strongly Agree

2 - Agree

3 - Neutral

4 - Disagree

5 - Strongly Disagree

6 - Does not apply

5. Using Microsoft Word or Google Docs for this task improves my performance.

1 - Strongly Agree

2 - Agree

3 - Neutral

4 - Disagree

5 - Strongly Disagree

6 - Does not apply

6. Using Microsoft Word or Google Docs enables me to finish the task more quickly.

1 - Strongly Agree

2 - Agree

3 - Neutral

4 - Disagree

5 - Strongly Disagree

6 - Does not apply

7. The interface of the Microsoft Word or Google Docs was clear and understandable.

1 - Strongly Agree

2 - Agree

3 - Neutral

4 - Disagree

5 - Strongly Disagree

6 - Does not apply 
8. The interaction with Microsoft Word or Google Docs was flexible.

1 - Strongly Agree

2 - Agree

3 - Neutral

4 - Disagree

5 - Strongly Disagree

6 - Does not apply

9. Learning to use Microsoft Word or Google Docs was easy to me.

1 - Strongly Agree

2 - Agree

3 - Neutral

4 - Disagree

5 - Strongly Disagree

6 - Does not apply

10. What implementation of collaborative writing do you prefer?

1 - Track changes implemented by MS Word

2 - Revisions implemented by Google Docs

3 - Neither

11. Why do you prefer track changes or revisions implementations of collaborative writing?

12. Do you prefer asynchronous (same time) collaborative writing or synchronous (different times)) collaborative writing?

1 - Asynchronous

2 - Synchronous

3 - Neither

13. Why do you prefer asynchronous (different times) collaborative writing or synchronous (same time) collaborative writing? 


\section{Suggestions}

1. What do you like the best about Microsoft Word when using track changes or Google Docs when using revisions history?

2. What do you like the least about Microsoft Word when using track changes or Google Docs when using revision history?

3. How would you suggest we improve the interface of Microsoft Word when using track changes or Google Docs when using revisions history? 


\section{APPENDIX D}

\section{Usability Study Posttest Survey}

(After completion of the interface prototype - usability study)

\section{General observations}

1. I feel this task was easy to complete?

1 - Strongly Agree

2 - Agree

3 - Neutral

4 - Disagree

5 - Strongly Disagree

6 - Does not apply

\section{Computer Experience}

1. My years of experience using a desktop or laptop computer helped me complete the task.

1 - Strongly Agree

2 - Agree

3 - Neutral

4 - Disagree

5 - Strongly Disagree

6 - Does not apply

\section{Opinions}

1. I am satisfied with the time I took to complete the tasks using the interface prototype.

1 - Strongly Agree

2 - Agree

3 - Neutral

4 - Disagree

5 - Strongly Agree 
2. I am satisfied with the result of my work.

1 - Strongly Agree

2 - Agree

3 - Neutral

4 - Disagree

5 - Strongly Disagree

6 - Does not apply

3. I was able to finish my task using the interface prototype without any problem.

1 - Strongly Agree

2 - Agree

3 - Neutral

4 - Disagree

5 - Strongly Disagree

6 - Does not apply

4. Assuming I have access to this interface prototype, I would use it on a regular basis in the future when I am doing similar tasks.

1 - Strongly Agree

2 - Agree

3 - Neutral

4 - Disagree

5 - Strongly Disagree

6 - Does not apply

5. Using the interface prototype for this task improves my performance.

1 - Strongly Agree

2 - Agree

3 - Neutral

4 - Disagree

5 - Strongly Disagree

6 - Does not apply 
6. Using the interface prototype enables me to finish the task more quickly.

1 - Strongly Agree

2 - Agree

3 - Neutral

4 - Disagree

5 - Strongly Disagree

6 - Does not apply

7. The interface of the interface prototype was clear and understandable.

1 - Strongly Agree

2 - Agree

3 - Neutral

4 - Disagree

5 - Strongly Disagree

6 - Does not apply

8. The interaction with the interface prototype was flexible.

1 - Strongly Agree

2 - Agree

3 - Neutral

4 - Disagree

5 - Strongly Disagree

6 - Does not apply

9. Learning to use the interface prototype was easy to me.

1 - Strongly Agree

2 - Agree

3 - Neutral

4 - Disagree

5 - Strongly Disagree

6 - Does not apply 


\section{Suggestions}

1. What do you like the best about the interface prototype?

2. What do you like the least about the interface prototype?

3. How would you suggest we improve the interface of the interface prototype?

\section{Preference}

1. For collaborative writing, which application do you prefer?

1 - Interface prototype

2 - Microsoft Word

3 - Google Docs

4 - None

2. If you have a preference, why do you prefer your choice selected in the prior question? 


\section{APPENDIX E}

Table 38. Research Focus and Contributions

\begin{tabular}{|c|c|c|c|}
\hline Research & Area of Focus & Research Questions & $\begin{array}{l}\text { Contribution(s) } \\
\text { of the Research }\end{array}$ \\
\hline $\begin{array}{l}\text { Hampel (Hampel et } \\
\text { al., 1999) BIRC - } \\
\text { Integration of } \\
\text { Visually Impaired } \\
\text { in Virtual } \\
\text { Communities }\end{array}$ & $\begin{array}{l}\text { Accessible } \\
\text { interface prototype }\end{array}$ & $\begin{array}{l}\text { Considering the } \\
\text { following issues: } \\
\text { spatial arrangements } \\
\text { of the GUI; } \\
\text { overlapping } \\
\text { windows; exploring } \\
\text { in 2-D space; too } \\
\text { much unstructured } \\
\text { information; and, } \\
\text { graphical messages } \\
\text { display only as icons, } \\
\text { how can the interface } \\
\text { be designed to } \\
\text { accommodate } \\
\text { accessibility? } \\
\text { Additionally, how } \\
\text { can the issues of } \\
\text { Braille displays } \\
\text { scrolling too fast and } \\
\text { the inability to save } \\
\text { content are solved? }\end{array}$ & $\begin{array}{l}\text { The use of an } \\
\text { Accessible } \\
\text { Interface prototype } \\
\text { was presented as a } \\
\text { solution to } \\
\text { generating text } \\
\text { content as opposed } \\
\text { to graphical } \\
\text { content and to } \\
\text { include key-press } \\
\text { control over the } \\
\text { scrolling actions of } \\
\text { a Braille display. } \\
\text { Additionally, key- } \\
\text { press combinations } \\
\text { were used to save } \\
\text { information. }\end{array}$ \\
\hline $\begin{array}{l}\text { Luk (Luk, et al., } \\
\text { 2000) Digital } \\
\text { Library access }\end{array}$ & Organizing content & $\begin{array}{l}\text { How can the interface } \\
\text { be organized to } \\
\text { enable Blind persons } \\
\text { to navigate the } \\
\text { interface? } \\
\text { How can Chinese } \\
\text { Blind person's access } \\
\text { digital libraries with } \\
\text { Braille input? }\end{array}$ & $\begin{array}{l}\text { The use of spatial } \\
\text { clusters of objects } \\
\text { near each other to } \\
\text { gain a richer } \\
\text { experience of the } \\
\text { interface and to } \\
\text { assist with } \\
\text { navigation. }\end{array}$ \\
\hline
\end{tabular}




\begin{tabular}{|c|c|c|c|}
\hline Research & Area of Focus & Research Questions & $\begin{array}{l}\text { Contribution(s) } \\
\text { of the Research }\end{array}$ \\
\hline $\begin{array}{l}\text { Takagi (Takagi, } \\
\text { 2000) Notes Reader }\end{array}$ & $\begin{array}{l}\text { Accessible } \\
\text { Interface prototype }\end{array}$ & $\begin{array}{l}\text { Can a tool be } \\
\text { developed to enable } \\
\text { blind users' access to } \\
\text { their email and } \\
\text { documents, since it is } \\
\text { very hard for blind } \\
\text { users to access } \\
\text { applications by using } \\
\text { screen readers? }\end{array}$ & $\begin{array}{l}\text { Accessible } \\
\text { Interface prototype } \\
\text { provided insight to } \\
\text { designing } \\
\text { collaborative } \\
\text { applications and } \\
\text { screen reader } \\
\text { applications. } \\
\text { Simplify the } \\
\text { interface (ease of } \\
\text { use), provide } \\
\text { keyboard-only (no } \\
\text { need for a mouse) } \\
\text { access, and text-to- } \\
\text { speech output } \\
\text { where a few design } \\
\text { considerations. }\end{array}$ \\
\hline $\begin{array}{l}\text { Thiessen (Thiessen } \\
\text { \& Chen, 2007) Reef } \\
\text { Chat Ajax Live } \\
\text { Regions }\end{array}$ & $\begin{array}{l}\text { Implementing } \\
\text { WAI-ARIA }\end{array}$ & $\begin{array}{l}\text { How can WAI-ARIA } \\
\text { be implemented to } \\
\text { make AJAX "Live" } \\
\text { Regions accessible } \\
\text { for blind persons? }\end{array}$ & $\begin{array}{l}\text { Accessible } \\
\text { Interface } \\
\text { prototypes can } \\
\text { include parameter- } \\
\text { driven markup to } \\
\text { control AJAX } \\
\text { "Live" Regions, so } \\
\text { the content is not } \\
\text { arbitrarily } \\
\text { displayed. }\end{array}$ \\
\hline $\begin{array}{l}\text { Tse (Tse et al., } \\
\text { 2007) Multi-modal } \\
\text { digital table }\end{array}$ & $\begin{array}{l}\text { Multiple } \\
\text { modalities }\end{array}$ & $\begin{array}{l}\text { Can hand gestures } \\
\text { and voice commands } \\
\text { help persons with } \\
\text { disabilities } \\
\text { manipulate content? }\end{array}$ & $\begin{array}{l}\text { Non-visual } \\
\text { modalities, hand } \\
\text { gestures and voice } \\
\text { commands, can } \\
\text { provide } \\
\text { accessibility to } \\
\text { content for persons } \\
\text { with disabilities. }\end{array}$ \\
\hline
\end{tabular}




\begin{tabular}{|c|c|c|c|}
\hline Research & Area of Focus & Research Questions & $\begin{array}{l}\text { Contribution(s) } \\
\text { of the Research }\end{array}$ \\
\hline $\begin{array}{l}\text { Watanabe } \\
\text { (Watanabe et al., } \\
\text { 2007) VoiceBlog }\end{array}$ & $\begin{array}{l}\text { Implementing } \\
\text { WCAG }\end{array}$ & $\begin{array}{l}\text { How can a } \\
\text { universally designed } \\
\text { voice browser be } \\
\text { designed, to work } \\
\text { with structured } \\
\text { information like } \\
\text { blogs and assist blind } \\
\text { users in interacting in } \\
\text { blog communities? }\end{array}$ & $\begin{array}{l}\text { Watanabe } \\
\text { illustrated the need } \\
\text { to simplify the user } \\
\text { interface, to reduce } \\
\text { the memory load } \\
\text { for blind users, and } \\
\text { to present content } \\
\text { aurally for blind } \\
\text { users. }\end{array}$ \\
\hline $\begin{array}{l}\text { Watters (Watters et } \\
\text { al., 2005) Web } \\
\text { Discussion Boards }\end{array}$ & $\begin{array}{l}\text { Implementing } \\
\text { WCAG }\end{array}$ & $\begin{array}{l}\text { How can Web } \\
\text { Discussion Boards be } \\
\text { modified to be } \\
\text { WCAG compliant? }\end{array}$ & $\begin{array}{l}\text { Essentially, IBM's } \\
\text { WAI Framework } \\
\text { can provide text- } \\
\text { to-speech } \\
\text { capabilities for } \\
\text { Web pages without } \\
\text { the need for screen } \\
\text { readers. }\end{array}$ \\
\hline $\begin{array}{l}\text { Winberg (Winberg, } \\
\text { 2004) Cooperative } \\
\text { Interfaces for the } \\
\text { Visually Impaired }\end{array}$ & $\begin{array}{l}\text { Multiple } \\
\text { modalities }\end{array}$ & $\begin{array}{l}\text { How can interface } \\
\text { design provided } \\
\text { access to all of the } \\
\text { functionality } \\
\text { available to the } \\
\text { sighted users using } \\
\text { alternative non-visual } \\
\text { methods, like } \\
\text { auditory and tactile } \\
\text { modalities? }\end{array}$ & $\begin{array}{l}\text { Multiple and } \\
\text { alternative modes } \\
\text { for input and } \\
\text { output are } \\
\text { necessary for } \\
\text { persons with } \\
\text { disabilities to gain } \\
\text { a rich experience } \\
\text { when interacting } \\
\text { with computer } \\
\text { interfaces. }\end{array}$ \\
\hline $\begin{array}{l}\text { Woodfine } \\
\text { (Woodfine et al., } \\
\text { 2008) Text-based e- } \\
\text { learning and } \\
\text { dyslexia }\end{array}$ & $\begin{array}{l}\text { Synchronous e- } \\
\text { learning } \\
\text { environment. }\end{array}$ & $\begin{array}{l}\text { How can persons } \\
\text { with Dyslexia keep } \\
\text { pace in a } \\
\text { synchronous e- } \\
\text { learning } \\
\text { environment? }\end{array}$ & $\begin{array}{l}\text { Synchronous } \\
\text { communications } \\
\text { are barriers for } \\
\text { persons with } \\
\text { disabilities who } \\
\text { may not be able to } \\
\text { keep pace in such } \\
\text { an environment. } \\
\text { Further research in } \\
\text { this area is needed. }\end{array}$ \\
\hline
\end{tabular}




\section{APPENDIX F}

Table 39. Collaborative Accessibility Research Summary

\begin{tabular}{|c|c|c|c|c|c|c|c|}
\hline Research & $\begin{array}{l}\text { Time / } \\
\text { Space }\end{array}$ & Type & $\begin{array}{l}\text { Disabilities } \\
\text { considered }\end{array}$ & Accessibility & $\begin{array}{c}\text { Input } \\
\text { support }\end{array}$ & $\begin{array}{c}\text { Output } \\
\text { forms }\end{array}$ & Solutions \\
\hline $\begin{array}{l}\text { Hampel (Hampel } \\
\text { et al., 1999) } \\
\text { BIRC - } \\
\text { Integration of } \\
\text { Visually } \\
\text { Impaired in } \\
\text { Virtual } \\
\text { Communities }\end{array}$ & $\begin{array}{l}\text { Different } \\
\text { place / } \\
\text { same time }\end{array}$ & Chat & $\begin{array}{l}\text { Persons who } \\
\text { are blind }\end{array}$ & $\begin{array}{l}\text { Spatial } \\
\text { arrangements of } \\
\text { the GUI - } \\
\text { difficult } \\
\text { conversion to } \\
\text { Braille, } \\
\text { overlapping } \\
\text { windows, } \\
\text { exploring 2-D } \\
\text { information } \\
\text { space, too much } \\
\text { unstructured } \\
\text { information, } \\
\text { messages are } \\
\text { graphical }\end{array}$ & Keyboard & $\begin{array}{l}\text { Braille } \\
\text { display, } \\
\text { auditory - } \\
\text { speech }\end{array}$ & $\begin{array}{l}\text { Assistive } \\
\text { tool, } \\
\text { structured } \\
\text { content, } \\
\text { Braille and } \\
\text { speech }\end{array}$ \\
\hline $\begin{array}{l}\text { Luk (Luk, et al., } \\
\text { 2000) Digital } \\
\text { Library access }\end{array}$ & $\begin{array}{l}\text { Different } \\
\text { place / } \\
\text { different } \\
\text { time }\end{array}$ & $\begin{array}{l}\text { Digital } \\
\text { library }\end{array}$ & $\begin{array}{l}\text { Persons who } \\
\text { are blind }\end{array}$ & $\begin{array}{l}\text { Orientation, } \\
\text { spatial } \\
\text { relationships are } \\
\text { not explicit, too } \\
\text { many details, } \\
\text { sequential } \\
\text { presentation of } \\
\text { information, } \\
\text { precise retrieval } \\
\text { of information }\end{array}$ & $\begin{array}{l}\text { Keyboard, } \\
\text { special } \\
\text { characters } \\
\text { to represent } \\
\text { Braille } \\
\text { code }\end{array}$ & $\begin{array}{l}\text { Braille } \\
\text { display, } \\
\text { auditory - } \\
\text { speech }\end{array}$ & 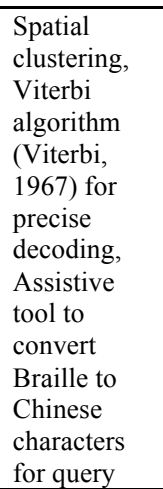 \\
\hline $\begin{array}{l}\text { Takagi (Takagi, } \\
\text { 2000) Notes } \\
\text { Reader }\end{array}$ & $\begin{array}{l}\text { Different } \\
\text { place / } \\
\text { different } \\
\text { time }\end{array}$ & E-mail & $\begin{array}{l}\text { Persons who } \\
\text { are blind }\end{array}$ & $\begin{array}{l}\text { Highly visual } \\
\text { graphical } \\
\text { interfaces, GUIs } \\
\text { are not } \\
\text { conducive to } \\
\text { screen readers }\end{array}$ & Keyboard & $\begin{array}{l}\text { Auditory - } \\
\text { speech, } \\
\text { Structured } \\
\text { list of e- } \\
\text { mail, } \\
\text { organized } \\
\text { inbox and } \\
\text { outbox }\end{array}$ & $\begin{array}{l}\text { Assistive } \\
\text { tool, } \\
\text { structured } \\
\text { content, text- } \\
\text { to-speech } \\
\text { using Speech } \\
\text { API }\end{array}$ \\
\hline $\begin{array}{l}\text { Thiessen } \\
\text { (Thiessen \& } \\
\text { Chen, 2007) } \\
\text { Reef Chat Ajax } \\
\text { Live Regions }\end{array}$ & $\begin{array}{l}\text { Different } \\
\text { place / } \\
\text { same time }\end{array}$ & Chat & $\begin{array}{l}\text { Persons who } \\
\text { are blind }\end{array}$ & $\begin{array}{l}\text { New content } \\
\text { appearing } \\
\text { arbitrarily, } \\
\text { instead of in a } \\
\text { linear manner }\end{array}$ & Keyboard & $\begin{array}{l}\text { Auditory - } \\
\text { speech }\end{array}$ & $\begin{array}{l}\text { Assistive } \\
\text { tool, } \\
\text { structured } \\
\text { content, text- } \\
\text { to-speech, } \\
\text { WAI-ARIA, } \\
\text { parameter- } \\
\text { driven } \\
\text { message } \\
\text { queuing }\end{array}$ \\
\hline
\end{tabular}




\begin{tabular}{|c|c|c|c|c|c|c|c|}
\hline Research & $\begin{array}{l}\text { Time / } \\
\text { Space }\end{array}$ & Type & $\begin{array}{l}\text { Disabilities } \\
\text { considered }\end{array}$ & Accessibility & $\begin{array}{c}\text { Input } \\
\text { support }\end{array}$ & $\begin{array}{l}\text { Output } \\
\text { forms }\end{array}$ & Solutions \\
\hline $\begin{array}{l}\text { Tse (Tse et al., } \\
\text { 2007) Multi- } \\
\text { modal digital } \\
\text { table }\end{array}$ & $\begin{array}{l}\text { Same place } \\
\text { / same time }\end{array}$ & $\begin{array}{l}\text { Digital } \\
\text { Table }\end{array}$ & None & Not considered & $\begin{array}{l}\text { Hand } \\
\text { Gestures } \\
\text { and voice } \\
\text { commands }\end{array}$ & $\begin{array}{l}\text { Digital } \\
\text { table } \\
\text { display }\end{array}$ & $\begin{array}{l}\text { Not } \\
\text { considered }\end{array}$ \\
\hline $\begin{array}{l}\text { Watanabe } \\
\text { (Watanabe et al., } \\
\text { 2007) VoiceBlog }\end{array}$ & $\begin{array}{l}\text { Different } \\
\text { place / } \\
\text { different } \\
\text { time }\end{array}$ & $\mathrm{B} \log$ & $\begin{array}{l}\text { Persons who } \\
\text { are blind }\end{array}$ & $\begin{array}{l}\text { Memorizing } \\
\text { words as they are } \\
\text { spoken, causes } \\
\text { listening and } \\
\text { pace issues, } \\
\text { cognitive } \\
\text { overload }\end{array}$ & $\begin{array}{l}\text { Keyboard } \\
\text { shortcuts }\end{array}$ & $\begin{array}{l}\text { Auditory - } \\
\text { speech, } \\
\text { eliminated } \\
\text { redundant } \\
\text { links }\end{array}$ & $\begin{array}{l}\text { Assistive } \\
\text { tool, } \\
\text { structured } \\
\text { content, } \\
\text { W3C } \\
\text { WCAG } \\
\text { Universal } \\
\text { design, Web- } \\
\text { based, built- } \\
\text { in, text-to- } \\
\text { speech } \\
\end{array}$ \\
\hline $\begin{array}{l}\text { Watters (Watters } \\
\text { et al., 2005) Web } \\
\text { Discussion } \\
\text { Boards }\end{array}$ & $\begin{array}{l}\text { Different } \\
\text { place / } \\
\text { same time }\end{array}$ & WebCT & $\begin{array}{l}\text { Persons who } \\
\text { are blind }\end{array}$ & $\begin{array}{l}\text { Graphical } \\
\text { content, tables } \\
\text { and frames } \\
\text { without } \\
\text { descriptions, } \\
\text { support keyboard } \\
\text { control, support } \\
\text { text-to-speech }\end{array}$ & $\begin{array}{l}\text { Keyboard } \\
\text { shortcuts }\end{array}$ & $\begin{array}{l}\text { Auditory } \\
\text { via screen } \\
\text { reader, list } \\
\text { organizatio } \\
\mathrm{n} \text { of the } \\
\text { text, text- } \\
\text { based. }\end{array}$ & $\begin{array}{l}\text { Assistive } \\
\text { tool or } \\
\text { proxy, apply } \\
\text { W3C } \\
\text { WCAG by } \\
\text { rewriting } \\
\text { web pages, } \\
\text { remove } \\
\text { JavaScript - } \\
\text { pop-ups }\end{array}$ \\
\hline $\begin{array}{l}\text { Winberg } \\
\text { (Winberg, 2004) } \\
\text { Cooperative } \\
\text { Interfaces for the } \\
\text { Visually } \\
\text { Impaired }\end{array}$ & $\begin{array}{l}\text { Same place } \\
\text { / same time }\end{array}$ & $\begin{array}{l}\text { Collabor } \\
\text { ative } \\
\text { manipul } \\
\text { ation }\end{array}$ & $\begin{array}{l}\text { Persons who } \\
\text { are blind }\end{array}$ & $\begin{array}{l}\text { Provide access to } \\
\text { functionality the } \\
\text { same as visually } \\
\text { able, enable } \\
\text { manipulation and } \\
\text { exploration }\end{array}$ & $\begin{array}{l}\text { Mouse } \\
\text { (with } \\
\text { auditory } \\
\text { tones) }\end{array}$ & $\begin{array}{l}\text { Auditory } \\
\text { tones }\end{array}$ & $\begin{array}{l}\text { Assistive } \\
\text { tool based on } \\
\text { auditory } \\
\text { signals }\end{array}$ \\
\hline $\begin{array}{l}\text { Woodfine } \\
\text { (Woodfine et al., } \\
\text { 2008) Text-based } \\
\text { e-learning and } \\
\text { dyslexia }\end{array}$ & $\begin{array}{l}\text { Different } \\
\text { place / } \\
\text { same time }\end{array}$ & $\begin{array}{l}\text { E- } \\
\text { learning }\end{array}$ & Dyslexia & $\begin{array}{l}\text { Fast-paced } \\
\text { synchronous } \\
\text { communication }\end{array}$ & $\begin{array}{l}\text { Not } \\
\text { considered }\end{array}$ & $\begin{array}{l}\text { Not } \\
\text { considered }\end{array}$ & $\begin{array}{l}\text { Assistance } \\
\text { from a tutor } \\
\text { or Mediator, } \\
\text { suggests } \\
\text { some sort of } \\
\text { assistance } \\
\text { "Reasonable } \\
\text { Adjustments } \\
\text { " }\end{array}$ \\
\hline
\end{tabular}




\section{APPENDIX G}

\section{USABILITY STUDY INFORMED CONSENT FORM}

\section{Dear Participant,}

My name is John G. Schoeberlein and I am a doctoral student in the Department of Computer and Information Sciences at Towson University. As part of the research for my doctoral thesis, I will be conducting usability studies to examine the access and use of collaborative writing applications.

Participation in this study is voluntary and you may discontinue your participation in the project at any time. If you choose to participate in this study, you will be asked to complete a short survey before the study and after the study. The surveys are available on an accessible web site (surveygizmo.com). It is not necessary to answer every question.

During the usability study, you will be asked to complete some basic editing tasks (add, change and delete content) and searching tasks of a sample document. Audio and video recordings of your interactions with the computer interface will be recorded. The video device will be directed at the computer screen to record the interface interactions and to maintain your anonymity. 
Your decision whether or not to participate in the project or to withdraw from the project at any time will not affect you in any way.

If you do choose to participate in the study, your participation will be completely confidential.

If you have any questions about the project, you may contact me at (410) 9311443, my faculty advisor, Dr. Yuanqiong (Kathy) Wang at (410) 704-2104, or Towson University's Institutional Review Board for the Protection of Human Participants, at (410) 704-2236.

A copy of the results of the survey, reported in aggregate form, will be available to you upon completion of my project, if you would like to see it.

Thank you for your time. 


\section{PRINCIPAL INVESTIGATOR: John Schoeberlein (Doctoral Student)}

PHONE: (410) 931-1443

Title of the Project: Collaborative Writing for the Blind

Purpose of the Study:

The purpose of this study is to identify accessibility and usability issues encountered when persons who are blind collaborate to co-author documents and utilizes track changes to identify revisions. The project directors hope to use the information obtained from this study will influence other developers and researchers to consider alternative approaches to collaborative writing.

Procedures:

Participants will attend a usability study session to examine the collaborative features of Microsoft Word and Google Docs.

Risks/Discomfort:

There are no known risks associated with participation in the study. Confidentiality of the data will be fully protected. Should the participation become distressing to you, it will be terminated immediately. 
Age Restriction:

Participants must be 18 years of age or older. By consenting, you are acknowledging that you are 18 years of age or older.

Benefits:

The following benefits are expected from my participation: opportunity to contribute to the understanding of the issues related to the accessibility and use of collaborative writing application and, to influence researchers to consider alternative approaches to developing interfaces for collaborative writing.

Alternatives to Participation:

Participation in this study is voluntary. You are free to withdraw or discontinue participation at any time. A decision to withdraw from the study will not affect you in any way. 
Confidentiality:

All information collected during the study period will be kept strictly confidential. You will be identified through identification numbers. No publications or reports from this project will include identifying information on any participant. If you agree to join this study, please sign your name below.

I have read and understood the information on this form.

I have had the information on this form explained to me.

Print Name

Subject's Signature Date

Witness to Consent Procedures Date

Principal Investigator Date

If you have any questions regarding this study please contact Dr. Yuanqiong Wang at (410) 704-2104 or John Schoeberlein at (410) 931-1443 or the Institutional Review Board, Office of University Research Services, 8000 York Road, Towson University, Towson, Maryland 21252; phone (410) 704-2236. 


\section{APPENDIX H}

\section{Usability Study Pretest Questionnaire \\ Demographics}

1. Gender:

1 - Male

2 - Female

2. Age range:

$$
\begin{aligned}
& 1-18 \text { and over } \\
& 2-19-29 \\
& 3-30-39 \\
& 4-40-49 \\
& 5-50-5 \\
& 6-60 \text { and over }
\end{aligned}
$$

\section{Computer Experience}

3. Approximately how many years have you been using a desktop or laptop computer?

$$
\begin{aligned}
& 1-\text { Less than } 1 \\
& 2-1 \text { to } 5 \\
& 3-6 \text { to } 10 \\
& 4-11 \text { to } 20 \\
& 5-21 \text { to } 30 \\
& 6-\text { Over } 30
\end{aligned}
$$

4. How frequently do you use a desktop or laptop computer?

1 - Daily

2 - Several times a week (two or more times)

3 - Weekly

4 - Several times a month

5 - Monthly 
5. When you use the computer, what is the primary use?

1 - Work

2 - Study

3 - Entertainment

4 - Other

6. Did you have any previous knowledge of any screen reader software?

1 - Yes

2 - No

If your answer to question number 6 is yes, which screen reader do you use?

If your answer to question number 6 is yes, approximately how many years of experience do you have using a screen reader?

$$
\begin{aligned}
& 1 \text { - Less than } 1 \\
& 2-1 \text { to } 5 \\
& 3-6 \text { to } 10 \\
& 4-11 \text { to } 20 \\
& 5-21 \text { to } 30 \\
& 6-\text { Over } 30
\end{aligned}
$$

If your answer to question number 6 is yes, how frequently do you use the screen reader?

1 - Daily

2 - Several times a week (two or more times)

3 - Weekly

4 -Several times a month

5 - Monthly 


\section{Collaborative Writing Experience}

7. Approximately how long have you worked with Microsoft Word?

$$
\begin{aligned}
& 1 \text { - Less than } 1 \text { year } \\
& 2 \text { - } 1 \text { to } 5 \text { years } \\
& 3-6 \text { to } 10 \text { years } \\
& 4-11 \text { to } 20 \text { years } \\
& 5 \text { - } 21 \text { to } 30 \text { years } \\
& 6 \text { - Over } 30 \text { years } \\
& \text { 7- Does not apply }
\end{aligned}
$$

If question 7 does not apply, skip questions 8, 9 and 10.

8. How frequently do you use Microsoft Word?

$$
\begin{aligned}
& 1 \text { - Daily } \\
& 2 \text { - Several times a week (two or more times) } \\
& \text { 3- Weekly } \\
& 4 \text { - Several times a month } \\
& 5 \text { - Monthly }
\end{aligned}
$$

9. Do you use the collaborative writing features (track changes) of Microsoft Word?

10. Can you identify any difficulty you have encountered, if any, when interacting with the collaborative features of Microsoft Word?

11. Approximately how long have you worked with Google Docs?

$$
\begin{aligned}
& 1 \text { - Less than } 1 \text { year } \\
& 2 \text { - } 1 \text { to } 5 \text { years } \\
& 3 \text { - } 6 \text { to } 10 \text { years } \\
& 4-11 \text { to } 20 \text { years } \\
& 5 \text { - } 21 \text { to } 30 \text { years } \\
& 6 \text { - Over } 30 \text { years } \\
& \text { 7- Does not apply }
\end{aligned}
$$

If question 11 does not apply, skip questions 12, 13 and 14. 
12. How frequently do you use the Google Docs?

$$
\begin{aligned}
& 1 \text { - Daily } \\
& 2 \text { - Several times a week (two or more times) } \\
& \text { 3-Weekly } \\
& 4 \text { - Several times a month } \\
& 5 \text { - Monthly }
\end{aligned}
$$

13. Do you use the collaborative writing features (revisions history) of Google Docs?

14. Can you identify any difficulty you have encountered, if any, when interacting with the collaborative features of Google Docs?

Please take notes or talk aloud during the study, if you feel frustrated or have questions. 


\section{APPENDIX I}

\section{Usability Study Microsoft Word Track Changes Script}

Start time

Task number 1: Open the document and start track changes

- Open the document, Amendments of the United States Constitution.docx.

- Press the following keys, control shift E (Windows) or command shift E (Mac), to turn on track changes.

End time 
Start time

Task number 2: Add track changes

- Search for, "Amendment I"

- Add the following text to the end of this amendment, "or the right of the people peaceably to assemble, and to petition the government for a redress of grievances."

- Save the document

- Search for, "Amendment VII"

- Add the following text to the end of this amendment, "than according to the rules of the common law."

- Save the document

- Search for, "Amendment VIII"

- Add the following text to the end of this amendment, "nor cruel and unusual punishments inflicted."

- Save the document

End time 
Start time

Task number 3: Delete text

- Search for, "Amendment VII"

- Delete the following text, "In suits at common law"

- Save the document

End time

Start time

Task number 4: Change text

- Search for, "well regulated"

- Change the text to, "well-regulated"

- Save the document

End time

Start time

Task number 5: Add a comment

Add a comment of your choice to Amendment II.

Save the document

End time 
Start time

Task number 6: Search for track changes and comments

- Search the entire document for all track changes.

End time

Start time

Task number 7: Accept a track change

- Search for the changed text, "nor cruel and unusual punishments inflicted."

- Accept the change

- Save the document

End time

Start time

Task number 8: Reject a track change

- Search for the deleted text, "In suits at common law," in Amendment VII

- Reject the change

- Save the document

End time 
Start time

Task number 9: Accept or Reject remaining changes

- Search for the track changes and accept or reject each change found

- Save the document

End time 


\section{APPENDIX J}

\section{Usability Study Document}

\section{Amendments of the United States Constitution}

\section{Amendment I}

Congress shall make no law respecting an establishment of religion, or prohibiting the free exercise thereof; or abridging the freedom of speech, or of the press;

\section{Amendment II}

A well-regulated militia, being necessary to the security of a free state, the right of the people to keep and bear arms, shall not be infringed.

\section{Amendment III}

Amendment III insert new text No soldier shall, in time of peace be quartered in any house, without the consent of the owner, nor in time of war, but in a manner to be prescribed by law.

\section{Amendment IV}

The right of the people to be secure in their persons, houses, papers, and effects, against unreasonable searches and seizures, shall not be violated, and no warrants shall issue, but upon probable cause, supported by oath or affirmation, and particularly describing the place to be searched, and the persons or things to be seized.

\section{Amendment $\mathbf{V}$}

No person shall be held to answer for a capital, or otherwise infamous crime, unless on a presentment or indictment of a grand jury, except in cases arising in the land or naval forces, or in the militia, when in actual service in time of war or public danger; nor shall 
any person be subject for the same offense to be twice put in jeopardy of life or limb; nor shall be compelled in any criminal case to be a witness against himself, nor be deprived of life, liberty, or property, without due process of law; nor shall private property be taken for public use, without just compensation.

\section{Amendment VI}

In all criminal prosecutions, the accused shall enjoy the right to a speedy and public trial, by an impartial jury of the state and district wherein the crime shall have been committed, which district shall have been previously ascertained by law, and to be informed of the nature and cause of the accusation; to be confronted with the witnesses against him; to have compulsory process for obtaining witnesses in his favor, and to have the assistance of counsel for his defense.

\section{Amendment VII}

In suits at common law, where the value in controversy shall exceed twenty dollars, the right of trial by jury shall be preserved, and no fact tried by a jury, shall be otherwise reexamined in any court of the United States,

\section{Amendment VIII}

Excessive bail shall not be required, nor excessive fines imposed,

\section{Amendment IX}

The enumeration in the Constitution, of certain rights, shall not be construed to deny or disparage others retained by the people.

\section{Amendment X}

The powers not delegated to the United States by the Constitution, nor prohibited by it to the states, are reserved to the states respectively, or to the people. 


\section{APPENDIX K}

\section{Setup by Researcher}

\section{Login as ryanschoeberlein@gmail.com}

Upload document, Amendments of the United States Constitution.docx.

Invite. johngschoeberlein@gmail.com 


\section{Usability Study Google Docs Track Changes Script}

Start time

Task number 1: Login as

- johngschoeberlein agmail.com

End time

Start time

Task number 2: Open the document

- Open the document, Amendments of the United States Constitution.docx.

End time

Start time 
Task number 3: Add track changes

- Search for, "Amendment I"

- Add the following text to the end of this amendment, "or the right of the people peaceably to assemble, and to petition the government for a redress of grievances."

- Search for, "Amendment VII"

- Add the following text to the end of this amendment, "than according to the rules of the common law."

- Search for, "Amendment VIII"

- Add the following text to the end of this amendment, "nor cruel and unusual punishments inflicted."

End time

Start time

Task number 4: Delete text

- Search for, "Amendment VII"

- Delete the following text, "In suits at common law"

End time 
Start time

Task number 5: Change text

- Search for, "well regulated"

- Change the text to, "well-regulated"

End time

Start time

Task number 6: Add a comment

Add a comment of your choice to Amendment II.

End time

Start time

Task number 7: Search for changes and comments in the revisions list

- Search the entire document for all track changes and accept or reject.

End time 


\section{APPENDIX L}

\section{Usability Study Prototype Script}

\section{Start time}

Task number 1: Open the document

- Open the document, Amendments of the United States Constitution.docx.

End time 
Start time

Task number 2: Add track changes

- Search for, "Amendment I"

- Add the following text to the end of this amendment, "or the right of the people peaceably to assemble, and to petition the government for a redress of grievances."

- Save the document

- Search for, "Amendment VII"

- Add the following text to the end of this amendment, "than according to the rules of the common law."

- Save the document

- Search for, "Amendment VIII"

- Add the following text to the end of this amendment, "nor cruel and unusual punishments inflicted."

- Save the document

End time 
Start time

Task number 3: Delete text

- Search for, "Amendment VII"

- Delete the following text, "In suits at common law"

- Save the document

End time

Start time

Task number 4: Change text

- Search for, "well regulated"

- Change the text to, "well-regulated"

- Save the document

End time

Start time

Task number 5: Add a comment

Add a comment of your choice to Amendment II.

Save the document

End time 
Start time

Task number 6: Search for changes and comments

- Search the entire document for all track changes.

End time

Start time

Task number 7: Accept a track change

- Search for the changed text, "nor cruel and unusual punishments inflicted."

- Accept the change

- Save the document

End time

Start time

Task number 8: Reject a track change

- Search for the deleted text, "In suits at common law," in Amendment VII

- Reject the change

- Save the document

End time 
Start time

Task number 9: Accept or Reject remaining changes

- Search for the track changes and accept or reject each change found

- Save the document

End time 


\section{APPENDIX M}

Table 40. Collaborative Research Timeline and Published Works

\begin{tabular}{|c|c|c|}
\hline Task & Timeline & Published Work(s) \\
\hline HCII 2009 & Summer 2009 (Complete) & $\begin{array}{l}\text { 1. Schoeberlein, J., Wang, } \\
\text { Y.: (2009) Evaluating } \\
\text { Groupware Accessibility, } \\
\text { LNCS. 5616, 414-423. } \\
\text { 2. Schoeberlein, J., Wang, } \\
\text { Y.: (2009) Groupware } \\
\text { Accessibility for Persons } \\
\text { with Disabilities, LNCS. } \\
\text { 5616, 404-413. }\end{array}$ \\
\hline $\begin{array}{l}\text { Towson University } \\
\text { Institutional Review } \\
\text { Board Application }\end{array}$ & Fall 2009 (Complete) & \\
\hline Focus Group Study (Pilot) & $\begin{array}{l}\text { Winter 2009, January } \\
2010 \text { (Complete) }\end{array}$ & \\
\hline $\begin{array}{l}\text { Data analysis and write } \\
\text { up of pilot focus group } \\
\text { study }\end{array}$ & Spring 2010 (Complete) & \\
\hline $\begin{array}{l}\text { Towson University } \\
\text { Institutional Review } \\
\text { Board Application }\end{array}$ & Summer 2010 (Complete) & \\
\hline Focus group study & $\begin{array}{l}\text { Summer - Fall } 2010 \\
\text { (Complete) }\end{array}$ & \\
\hline Group task field study & $\begin{array}{l}\text { Summer - Fall } 2010 \\
\text { (Complete) }\end{array}$ & \\
\hline $\begin{array}{l}\text { Data analysis and write } \\
\text { up of focus group study } \\
\text { and group task field } \\
\text { studies }\end{array}$ & Fall 2010 (Complete) & \\
\hline Proposal write up & $\begin{array}{l}\text { Spring - Fall } 2011 \\
\text { (Complete) }\end{array}$ & \\
\hline
\end{tabular}




\begin{tabular}{|c|c|c|}
\hline Task & Timeline & Published Work(s) \\
\hline HCII 2011 & Summer 2011 (Complete) & $\begin{array}{l}\text { 3. Schoeberlein, J., Wang, } \\
\text { and Y.: Examining the } \\
\text { Current State of Group } \\
\text { Support Accessibility: A } \\
\text { Focus Group Study, } \\
\text { Universal Access In } \\
\text { Human-Computer } \\
\text { Interaction. 6768, 272-281. } \\
\text { (2011). } \\
\text { 4. Schoeberlein, J., Wang, } \\
\text { and Y.: Examining the } \\
\text { Current State of Group } \\
\text { Support Accessibility: An } \\
\text { Expanded Study, } \\
\text { Communications in } \\
\text { Computer and Information } \\
\text { Science. 173, 389 - 393. } \\
\text { (2011). }\end{array}$ \\
\hline Dissertation Proposal & Spring 2012 (complete) & Proposal approved. \\
\hline $\begin{array}{l}\text { Towson University } \\
\text { Institutional Review } \\
\text { Board Application }\end{array}$ & Spring 2012 (Complete) & \\
\hline $\begin{array}{l}\text { Phase } 1 \text { - Baseline } \\
\text { usability study of MS } \\
\text { Word and Google Docs }\end{array}$ & Spring 2012 (Complete) & \\
\hline $\begin{array}{l}\text { Data analysis and write } \\
\text { up of the baseline } \\
\text { usability study }\end{array}$ & Spring 2012 (Complete) & \\
\hline ASSETS' 2012 & Fall 2012 (Complete) & $\begin{array}{l}\text { 5. Accessible Collaborative } \\
\text { Writing for the Blind: A } \\
\text { Usability Study. In: } \\
\text { Proceedings of ASSETS } \\
\text { 2012, 267-268, Boulder, } \\
\text { CO. (2012). }\end{array}$ \\
\hline $\begin{array}{l}\text { Phase } 2 \text { - Proposed } \\
\text { interface solution } \\
\text { development }\end{array}$ & $\begin{array}{l}\text { Summer - Fall } 2012 \\
\text { (Complete) }\end{array}$ & $\begin{array}{l}\text { CollaborateWithWord, } \\
\text { CollaborateWithWord2007. }\end{array}$ \\
\hline
\end{tabular}




\begin{tabular}{|l|l|l|}
\hline \multicolumn{1}{|c|}{ Task } & \multicolumn{1}{|c|}{ Timeline } & \multicolumn{1}{c|}{ Published Work(s) } \\
\hline $\begin{array}{l}\text { Phase 3 - Pilot usability } \\
\text { study of the proposed } \\
\text { interface }\end{array}$ & $\begin{array}{l}\text { Fall 2012 - Winter 2013 } \\
\text { (Complete) }\end{array}$ & \\
\hline $\begin{array}{l}\text { Data analysis and write } \\
\text { up of the pilot usability } \\
\text { study of the proposed } \\
\text { interface }\end{array}$ & $\begin{array}{l}\text { Fall 2012 - Winter 2013 } \\
\text { (Complete) }\end{array}$ & \\
\hline HCII 2013 & $\begin{array}{l}\text { Spring 2013 (Complete) } \\
\text { Presentation in July 2013 }\end{array}$ & $\begin{array}{l}\text { 6. Schoeberlein, J., Wang, } \\
\text { Y.: Providing an Accessible } \\
\text { Track Change Feature for } \\
\text { Persons Who Are Blind, } \\
\text { Universal Access In } \\
\text { Human-Computer } \\
\text { Interaction. (2013). }\end{array}$ \\
\hline $\begin{array}{l}\text { Phase 4 - Proposed } \\
\text { interface solution } \\
\text { enhancement }\end{array}$ & Spring 2013 (Complete) & $\begin{array}{l}\text { CollaborateWithWord, } \\
\text { CollaborateWithWord2007. }\end{array}$ \\
\hline $\begin{array}{l}\text { Phase 5 - Final usability } \\
\text { studies of the proposed } \\
\text { interface }\end{array}$ & Spring 2013 (Complete) & \\
\hline $\begin{array}{l}\text { Data analysis and write } \\
\text { up of the final usability } \\
\text { study of the proposed } \\
\text { interface }\end{array}$ & Spring 2013 (Complete) & $\begin{array}{l}\text { 7. Schoeberlein, J., Wang, } \\
\text { Y.: (2013) Improving } \\
\text { Collaborative Writing for } \\
\text { the Blind, ACM } \\
\text { SIGACCESS Article. } \\
\text { 8. Schoeberlein, J., Wang, } \\
\text { Y.: (2013) Usability } \\
\text { Evaluation of an Accessible } \\
\text { Collaborative Writing } \\
\text { Prototype for Blind Users, } \\
\text { Journal of Usability Studies. }\end{array}$ \\
\hline
\end{tabular}


NFB Support Letter

\title{
APPENDIX N
}

\author{
Marc Maurer, President
200 East Wells Street
at Jemigan Place
Baltimore, MD 21230
Phone 4106599314 Fax 410685 5653
www.nfb.org

National Federation

of the Blind

Thursday, June 17, 2010

Office of University Research Services

Towson University

8000 York Road

Towson, Maryland 21252

Office of University Research Services,

The National Federation of the Blind (NFB) Jernigan Institute is delighted to support by John Schoeberlein (Doctoral Student) and Dr. Yuanqiong (Kathy) Wang (Faculty Advisor) in their research into accessibility and usability issues of groupware tools. The NFB Jernigan Institute's Access Technology team will be happy to assist you in conducting a field study on this topic. Additionally, designated NFB staff will provide advice and assistance as needed.

The National Federation of the Blind Jernigan Institute exercises a high degree of selectivity when selecting organizational partners and granting our organizational endorsement. We believe that the work being done by Mr. Schoeberlein and Dr. Wang will benefit blind users and we look forward to working with them to make their research program a success.

I can be reached by phone at (410)659-9314, extension 2413.

Sincerely,

Anne Taylor

Director of Access Technology

Jernigan Institute

National Federation of the Blind

AT/cvg 


\section{Focus Group IRB}

alfixe of University Re-searrh Services

Tawsan Univensily Bo00 York Road Tiswson, MD 21252-0001

t. $410704-2236$ f. $410704-4494$

\section{APPENDIX 0}

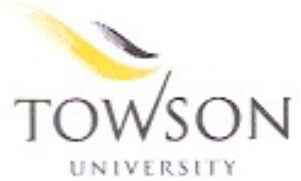

APPROVAL NUMBER: $\quad$ 10-A032

$\begin{array}{lll}\text { To: John } & \text { Schocberlein } \\ \text { 9 Chatterly Court } & \\ \text { Perry Hall } & \text { MD } & 21128\end{array}$

From: Institutional Review Board for the Proctection of Human Subjects Marcic Weinstein, Member

Date: $\quad$ Monday, January 11, 2010

JAN 112010

RE: $\quad$ Application for Approval of Research Involving the Use of Human Participants

Thank you for submitting an Application for Approval of Research Involving the Use of Human Participants to the Institutional Review Board for the Protection of Human Participants (IRB) at Towson University. The IRB hereby approves your proposal titled:

Evaluating Giroupware Accessibility for the Blind

If you should encounter any new risks, reactions, or injuries while conducting your research, please notify the IRB. Should your research cxtend beyond one year in duration, or should there be substantive changes in your rescarch protocol, you will need to submit another application for approval at that time.

We wish you every success in your rescarch project. If you have any questions, please call me at (410) 704-2236.

CC: Kathy Wang

File 


\section{Field Study IRB}

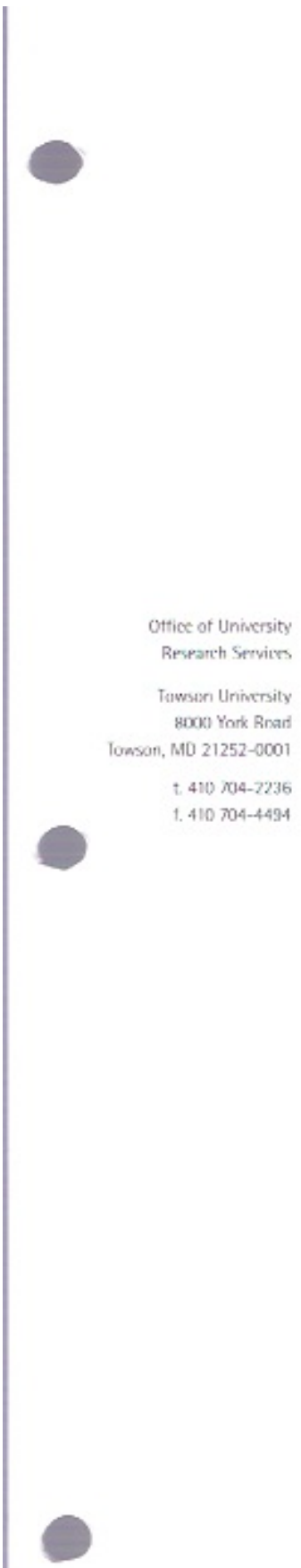

\section{APPENDIX P}

\section{TOW̌̀n \\ UNIVERITY}

\section{APPROVAL NUMBER: 11-A004}

To:

$$
\begin{aligned}
& \text { John Schocberlein } \\
& \begin{array}{ll}
9 \text { Chatterly Court } & \\
\text { Perry Hall } & \text { MD } 21128
\end{array}
\end{aligned}
$$

From: Institutional Review Board for the Proctection of Human Subjects Patricia Alt, Member

Date: Thursday, July 01, 2010

RE:

Application for Approval of Research Involving the Use of Human Participants

Thank you for submitting an $\Lambda$ pplication for Approval of Research Involving the Use of Human Participants to the Institutional Review Board for the Protection of Human Participants (IRB) at Towson University. The IRB hereby approves your proposal titled:

Livaluating Groupware Accessibility for the Blind (-a field study)

If you should encounter any new risks, reactions, or injuries while conducting your research, please notify the IRB. Should your research extend beyond one year in duration, or should there be substantive changes in your research protocol, you will need to submit another application for approval at that time.

We wish you every success in your research project. If you have any questions, please call me at (410) 704-2236.

$\mathrm{CC}$ : Yuanqiong Wang:

File 


\section{Usability Study IRB}

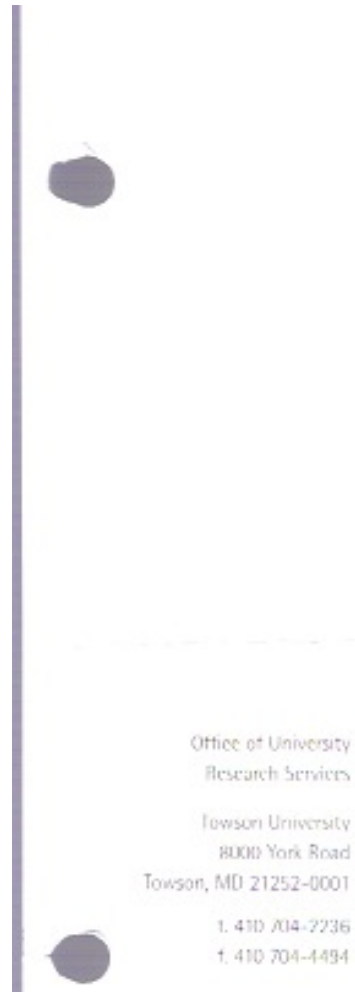

\section{APPENDIX Q}

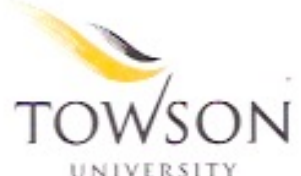

UNIVERSITY

\section{RENEWED APPROVAL NUMBER: 12-A060R1}

$\begin{array}{ll}\text { To: } & \text { John Schoeberlein } \\ \text { From: } & \text { Institutional Review Board for the Protection of Human } \\ & \text { Subjects, Justin Buckingham, Member } \\ \text { Date: } & \text { Monday, March 05.2012 } \\ \text { RE: } & \text { Application for Approval of Rescarch Involving the Use of } \\ & \text { Human Participants }\end{array}$

Thank you for completing the Annual Revicw Notice for Projects Involving IIuman Participants for the project titled:

Accessible Collaborative Writing for the Blind

Since you have indicated that your research project is still active, we are granting you a renewal of your approval. If you should encounter any new risks, reactions, or injuries while conducting your research, please notify the IRB. Should there be substantive changes in your research protocol, you will need to submit another application for approval at that time. This protocol will be reviewed again one year from this date of approval

We wish you every success in your research project. If you have any questions, please call me at (410) 704-2236.

CC: Kathy Wang

File 


\section{LIST OF REFERENCES}

1.Accessibility (2013). http://en.wikipedia.org/wiki/Accessibility

2.Adaptive Technology (2008) http://en.wikipedia.org/wiki/Adaptive technology> Accessed April 26, 2008.

3.AIM. (2008) http://dashboard.aim.com/aim Accessed July 9, 2008.

4.AIM Object Library. (2008) http://dev.aol.com/aim Accessed July 9, 2008.

5.AOL Company Overview (2009) http://corp.aol.com/about-aol/company-overview Accessed February 7, 2009.

6.Appelt, W. (1999). WWW Based Collaboration with BSCW System. Proceedings of SOFSEM'99, Springer Lecture Notes in Computer Science 1725, Milovy, Czech Republic, pages 66-78.

7.Arditi, A., \& Rosenthal, B. (1998). "Developing an objective definition of visual impairment." In Vision '96: Proceedings of the International Low Vision Conference (pp. 331-334). Madrid, Spain: ONCE.

8.Assistive Technologies for Persons with Dyslexia. (2013).

http://evengrounds.com/blog/assistive-technologies-for-persons-with-dyslexia.

9.ATRC Web Accessibility Checker (2009) http://checker.atrc.utoronto.ca/index.html Accessed February 22, 2009.

10.Augmentative and Alternative Communication (AAC) (2013).

http://en.wikipedia.org/wiki/Augmentative_and_alternative_communication 11.Authoring Tool Accessibility Guidelines (ATAG) 2.0. (2009).

http://www.w3.org/WAI/intro/atag.php Accessed July 10, 2009. 
12.Authoring Tool Accessibility Guidelines 2.0 (ATAG 2.0). (2013).

http://www.w3.org/TR/ATAG20/\#sc b244

13.Barrett, R., \& Maglio, P. (1999) Intermediaries: An approach to manipulating

information streams. IBM Systems Journal, 1999, 38, pages 629-641.

14. Bell Craft DeskBot (2008) www.bellcraft.com/deskbot Accessed September 14, 2008.

15.Bigham, Jeffrey P., Prince, Craig M., Ladner, Rechard D. (2008) Web Anywhere: A

Screen Reader On-the-go. In Proceedings W4A2008 - Technical, Beijing, China. April $22-23,2008$.

16.Bishup, J., Rama, J. (2007). A Survey and Comparison of CSCW Group support Applications. Proceedings of SAICSIT, pages 198-205.

17.Bishup, J., Danzfuss, T., Klazar, R., Rama, J., Tseoali, T. (2005). Nomad -

Collaborative group support for casually-connected communities. Technical

Report, Polelo Research Group, University of Pretoria.

18.Burgess, Robert G., (1995). In the Field: An Introduction to Field Research.

Routledge, London, 1995.

19.Buzzi, C., Buzzi, M., Leporini, B., Senette, C. (2008). Making Wikipedia Editing

Easier for the Blind. In Proceedings NordiCHI 2008, pages 423-426, October 20-22, 2008.

20.Buzzi, M.C., Buzzi, Mori, Giulio, M., Leporini, B., Penichet, V.: Accessing Google

Docs via Screen Reader, LNCS, 6179, 92-99. (2010).

21.Buzzi, M.C., Buzzi, Mori, Giulio, M., Leporini, B., Penichet, V.: Making “Google

Docs" User Interface More Accessible for Blind People, Proceedings of ADNTIIC'10, 20-29. (2010). 
22.Buzzi, M.C., Buzzi, M., Leporini, B., Mori, Giulio, Penichet, V.: Collaborative Editing for All: The Google Docs Example, LNCS, 6768, 165-174. (2011).

23.Chapuis, O., \& Roussel, N. (2007). Eye Point: Practical Pointing and Selection Using Gaze and Keyboard. In Proceedings of CHI '07, April 28 - May 3, 2007, ACM Press, pages 421-430.

24.Chong, C. Making your web site accessible to the visually impaired. http://www.nfb.org/nfb/Web_accessibility.asp?SnID=2. Accessed April 11, 2008. 25.Cisco WebEx, http://www.webex.com/ 26.Closed Caption (2008) http://en.wikipedia.org/wiki/Closed_caption Accessed April 26, 2008.

27.Cohen's Kappa (2010). http://www.experiment-resources.com/cohens-kappa.html. Accessed February 28, 2010.

28.Collaborative Software, http://en.wikipedia.org/wiki/Collaborative_software Accessed November 24, 2013.

29.Computer Accessibility (2008) http://en.wikipedia.org/wiki/Category:Computer_accessibility Accessed May 1, 2008. 30.Courage, C., Baxter, K.: Understanding Your Users. A Practical guide to user requirements. Methods, Tools, and Techniques. Morgan Kaufmann Publishers, San Francisco (2005).

31.Data Analysis, http://www.challenge.nm.org/kickoff/classes/what-is-data-analysis.pdf Accessed November 26, 2013.

32.Dorigo, Martin, Harriehausen, B.: Survey: Improving Document Accessibility from the Blind and Visually Impaired User's Point of View, LNCS, 6768, 129-135. (2011). 
33.Dojo (2006) Dojo the JavaScript Toolkit. January 15, 2006, http://www.dojotoolkit.org,

34.Ellis, C.A., Gibbs, S.J., Reim, G.L. (1991) Group support: Some issues and experiences, Communications of the ACM, 34, 1, January 1, 1991, PAGES 680-689.

35.Ext JS Web Toolkit. (2009). http://extjs.com/ Accessed September 23. 2009.

36.Field Research. (2011). http://en.wikipedia.org/wiki/Field_research. Accessed April 1, 2011.

37.FireFox Web Browser. (2009). http://www.mozilla.com/firefox/ Accessed September 23, 2009.

38.Freedom Scientific Pac Mate (2008)

http://www.freedomscientific.com/fs products/PACmate2.asp Accessed October 27, 2008.

39.Freedom Scientific JAWS (2008).

http://www.freedomscientific.com/fs_products/software_jaws.asp, Accessed April 11, 2008.

40.Fujitsu Web Accessibility Inspector (2008).

http://www.fujitsu.com/global/accessibility/assistance/wi/, Accessed April 11, 2008.

41.Goebert, B., \& Rosenthal, G. (2002). Beyond Listening: Learning the Secret Language of Focus Groups. New York: John Wiley.

42.Google Blog. (2008) http://googleblog.blogspot.com Accessed July 5, 2008.

43.Google Blogger Data API. (2008) http://code.google.com/apis/blogger/index.html Accessed July 5, 2008. 
44.Google Docs (2009)

https://www.google.com/accounts/ServiceLogin?service=writely\&passive $=$ true $\&$ nui $=1$ \&continue $=$ http $\% 3 \mathrm{~A} \% 2 \mathrm{~F} \% 2 \mathrm{Fdocs}$. google.com $\% 2 \mathrm{~F} \&$ followup $=\mathrm{http} \% 3 \mathrm{~A} \% 2 \mathrm{~F} \% 2 \mathrm{Fdocs}$. google.com\%2F\&ltmpl=homepage\&rm=false Accessed February 23, 2009.

45.Google Documents List Data API (2009)

http://code.google.com/apis/documents/overview.html. Accessed February 23, 2009.

46.Greenbaum, T. L.: The handbook for focus group research. $2^{\text {nd }}$ Edition. Sage

Publication. Thousand Oaks (1998).

47.Grounded Theory (2009). http://www.analytictech.com/mb870/introtoGT.htm Accessed September 12, 2009.

48.Group Systems, Inc. (2008) http://www.groupsystems.com/technology Accessed September 14, 2008.

49.Group Systems Think Tank (2008) http://www.groupsystems.com/thinktank Accessed September 14, 2008.

50.Group Systems Think Tank templates (2008) http://www.groupsystems.com/usingthinktank-emplates Accessed September 15, 2008.

51.GW Micro Braille Sense (2008) http://www.gwmicro.com/Braille_Sense/ Accessed October 27, 2008\.

52.Hampel, T., Keil-Slawik, R., Claassen B.G., Plohmann, F., Reimann, C. (1999). Pragmatic Solutions for Better Integration of the Visually Impaired in Virtual Communities. Proc. GROUP '99, Phoenix, AR, USA 1999, pages 258-266. 
53.Harrison, S.M. (2005) Opening the Eyes of Those Who Can See to the World of Those Who Can't: A Case Study. Proc. SIGCSE’05, St. Louis, Missouri, USA, February 2327, 2005, pages 22-26.

54.Hoffe, G.H., Lugt, H.T. (1997). CoCoDoc: A Framework for Collaborating compound document editing based on OpenDoc and CORBA. Proceedings IFIP/IEEE, Conference on Open Distributed Processing and Distributed Platforms, pages 15-33. 55.ICE faces Web Toolkit. (2009). http://www.icefaces.org/ Accessed September 23, 2009.

56.International Council of Ophthalmology. "International Standards: Visual Standards Aspects and Ranges of Vision Loss with Emphasis on Population Surveys." In the $29^{\text {th }}$ International Congress of Ophthalmology. Sidney, Australia, April 2002.

57.Jarvi, J., Martin, B., Pecci, I., Pietrzak, T., Raisamo, R., Rami, S. (2007) the MICOLE Architecture: Multimodal Support for Inclusion of Visually Impaired Children. In Proceedings of ICMI'07, pp. 193-200. Nagoya, Aichi, Japan. 58.Kiger, J.L., (1984). The Depth/Breadth Trade-off I the Design of Menu-Driven User Interfaces. The International Journal of Man-Machine Studies, 20, pages 201-213. 59. Kurasaki, K.S. (2000). Intercoder Reliability for Validating Conclusions Drawn from Open-Ended Interview Data. Field Methods, volume 12, No. 3, August 2000, pp 179184.

60.Lazar, Jonathan, Feng, Jinjuan Heidi, Hocheiser, H.: (2010) Research Methods in Human-Computer Interaction. West Sussex, United Kingdom. Wiley \& Sons. 61.Lazar, J. (2007) Universal Usability: Designing Computer Interfaces for Diverse Users. John Wiley \& Sons, Inc., Hoboken, NJ 07030, USA. 
62.Luk, R., Yeung, D., Lu, O., Leung, E., Li, S.Y., Leung, F. (2000) Digital Library Access for Chinese Visually Impaired. ACM 2000, pages 244-245.

63. McGrath, J.E. (1984) Groups: Interaction and Performance. Prentice-Hall, Inc., Englewood Cliffs, N.J., 1984.

64.Microsoft Corporation: Properties on Windows Forms Controls That Supports Accessibility Guidelines. http://msdn.microsoft.com/enus/library/880wbs24\%28v=vs.90\%29.aspx. Accessed March 6, 2011.

65. Microsoft Internet Explorer. (2009) http://www.microsoft.com/windows/internetexplorer/default.aspx Accessed September 23, 2009.

66.Microsoft Outlook. (2008) http://office.microsoft.com/en-us/outlook/default.aspx Accessed July 9, 2008.

67. Microsoft Outlook Object Library. (2008) http://msdn.microsoft.com/enus/library/aa189757(office.10).aspx Accessed July 9, 2008.

68.Microsoft SharePoint. (2007) April 3, 2008. http://www.microsoft.com/sharepoint/overview.mspx. 69.Microsoft SharePoint (2009) http://www.microsoft.com/Sharepoint/default.mspx. Accessed April 17, 2009.

70. Microsoft SharePoint Services 3.0: Software Development Kit (SDK) (2009) http://www.microsoft.com/downloads/details.aspx?familyid=05e0dd12-8394-402b8936-a07fe8afaffd\&displaylang=en. Accessed April 17, 2009.

71.Microsoft Visual Basic 2010 (2013). http://msdn.microsoft.com/enus/library/vstudio/2x7h1hfk(v=vs.100).aspx Accessed November 26, 2013. 
72.Microsoft Visual Studio 2010 (2013).

http://www.microsoft.com/visualstudio/eng/2013-preview

73.Microsoft Word Market share, (2013).

http://www.bgsu.edu/departments/english/cconline/lewis/worddom.html

74. Mikovec, Z., Vystrcil, J, Slavik, P. (2009) Web Toolkits Accessibility Study.

SIGACCESS Newsletter, Issue 94, pages 3-8.

75.MSDN Comments Collection. http://msdn.microsoft.com/enus/library/office/aa220940(v=office.11).aspx Accessed November 26, 2013.

76.MSDN Microsoft Visual Studio (2009). http://msdn.microsoft.com/enus/vstudio/default.aspx Accessed September 12, 2009.

77. MSDN Microsoft Word Command Bar. http://msdn.microsoft.com/enus/library/office/aa165324(v=office.10).aspx Accessed November 26, 2013.

78.MSDN Paragraphs Collection. http://msdn.microsoft.com/enus/library/office/aa679608(v=office.10).aspx Accessed November 26, 2013.

79. MSDN Revisions Collection. http://msdn.microsoft.com/enus/library/office/aa223078(v=office.11).aspx Accessed November 26, 2013. 80.National Federation of the Blind (2008) http://www.nfb.org . Accessed October 25, 2008.

81.National Federation of the Blind Lawsuit. (2013).

http://www.ecommercetimes.com/story/Google-Apps-Unfair-to-Blind-StudentsCharges-NFB-72078.html?wlc $=1300315852$

82.Nielsen Norman Group Seminar (2011).

http://www.nngroup.com/services/fieldstudies.html. Accessed April 1, 2011. 
83.Non-Visual Desktop Access (NVDA) (2009) http://www.nvda-project.org/ Accessed June 11, 2009.

84.Novell iFolder. (2007) April 3, 2008. http://www.novell.com/products/ifolder.

85. The Paciella Group Blog (2009). http://www.paciellogroup.com/blog/?p=89 Accessed September 22, 2009.

86.PHANToM Omin (2009). http://www.sensable.com/haptic-phantom-omni.htm.

Accessed July 7, 2009.

87.Qualitative Data Analysis (2009). ftp://ftp.qualisresearch.com/pub/qda.pdf. Accessed July 28, 2009.

88.Rehabilitation Act, Section 508 (1998)

http://www.section508.gov/index.cfm?FuseAction=content\&ID=12 Accessed May 1, 2009.

89.Schoeberlein, J., Wang, Y.: (2009) Groupware Accessibility for Persons with

Disabilities, LNCS. 5616, 404-413.

90.Schoeberlein, J., Wang, Y.: (2009) Evaluating Groupware Accessibility, Universal Access in Human-Computer Interaction. HCI International Conference, LNCS. 5616, 414-423, San Diego, CA. USA.

91.Schoeberlein, J., Wang, Y.: Examining the Current State of Group Support Accessibility: A Focus Group Study, Universal Access in Human-Computer Interaction. 6768, 272-281. (2011).

92.Schoeberlein, J., Wang, Y.: Examining the Current State of Group Support Accessibility: An Expanded Study, Communications in Computer and Information Science. 173, 389 - 393. (2011). 
93.Schoeberlein, J., Wang, J.: Accessible Collaborative Writing for the Blind: A Usability Study. In: Proceedings of ASSETS’2012, 267-268, Boulder, CO. (2012) 94.Schoeberlein, J., Wang, Y.: (2013) Providing an Accessible Track Change Feature for Persons Who Are Blind, In the Proceedings of HCII $201315^{\text {th }}$ International Conference on Human-Computer Interaction, UAHCI/HCII 2013, Part III, LNCS 8011, pp. 389398, July 21-26, 2013, Las Vegas, Nevada, USA.

95.Schultz, U., Vandenbosch, and B. (1998) Information Overload in a Group support Environment: Now You See It, Now You Don't. Journal of Organizational Computing and Electronic Commerce. Volume 8, Issue 2, June 1998, pages 127-148. 96.Section 508, 1194.21 Software Applications and Operating Systems. (2013). http://www.uspto.gov/about/offices/cio/section508/02software.jsp 97.Shneiderman, B., Plaisant, C. (2005) Designing the User Interface: Strategies for an Effective Human-Computer Interaction. $4^{\text {th }}$ edition, pp. 411-450. Addison Wesley, Boston.

98.Shneiderman, B., Plaisant, C. (2005) Designing the User Interface: Strategies for an Effective Human-Computer Interaction. $4^{\text {th }}$ edition, pp. 346-385. Addison Wesley, Boston.

99.Sorensonvrs. (2013). http://www.sorensonvrs.com/svrs/using_svrs 100.Speech Recognition (2008) http://en.wikipedia.org/wiki/Speech_recognition Accessed April 26, 2008. 101.Speech Synthesis (2008) http://en.wikipedia.org/wiki/Speech_synthesis Accessed April 26, 2008. 
102.Special Education Needs and Disabilities Act. (2001). Special Education Needs and Disabilities Act, The Queen's Printer of Acts of Parliament, The Stationary Office Limited, London, UK.

HTTP://WWW.HMSO.GOV.UK/ACTS/ACTS2001/20010010.HTM Accessed March 8, 2008.

103.SSPS, Inc. (2010). http://www.spss.com/ Accessed February 28, 2010.

104.SubEthaEdit. (2007) April 3, 2008. http://www.codingmonkeys.de/subethaedit. 105.Survey Gizmo, http://www.surveygizmo.com/

106.Takagi, H., Asakawa, C., Itoh, T. (2000). Non-Visual Group support Client: Notes Reader. Proc. Center on Disability Technology and Persons with Disabilities Conference, California State University.

107.Takagi, H., Saito, S., Fukuda, K., Asakawa, C. (2007) Analysis of Navigability of Web Applications for Improving Blind Usability. ACM Transactions on ComputerHuman Interactions. Volume 13, No. 3, Article 13.

108. Telecommunications Devices (2008)

http://en.wikipedia.org/wiki/Telecommunications devices for the deaf Accessed April $26,2008$.

109.Text on 9 Keys. (2009). http://www.t9.com/us/learn/ Accessed July 28, 2009. 110.TIKI WIKI, http://info.tiki.org/tiki-index.php Accessed November 26, 2013. 111.Tse, E., Shen, C., Greenberg, S. and Forlines, C. (2007). How Pairs Interact Over a Multimodal Digital Table. Proc. CHI 2007, ACM Press, 216-218. 
112.Thiessen, P., Chen, C. (2007) Ajax Live Regions: Chat as a Case Example.

Proceedings of the 2007 International World Wide Web Conference (W4A). May 0708, 2007.

113.Twitter (2009) http://twitter.com Accessed February 7, 2009.

114. Twitter API (2009) http://apiwiki.twitter.com Accessed February 7, 2009.

115.Umar, Amjad, (1997) Object-Oriented Client/Server Internet Environments. pp. 373380. Prentice Hall, Upper Saddle River, New Jersey.

116. Umar, Amjad, (1997) Object-Oriented Client/Server Internet Environments. pp. 52-

58. Prentice Hall, Upper Saddle River, New Jersey.

117.. U.S. Census Bureau. SEX BY EMPLOYMENT DISABILITY BY EMPLOYMENT

STATUS FOR THE CIVILIAN NONINSTITUTIONALIZED POPULATION 16 TO

64 , http://factfinder.census.gov/servlet/DTTable? $b \mathrm{bm}=\mathrm{y} \&$-state=dt\&-

ds_name $=$ ACS 2006 EST_G00_\&-_geoSkip $=0$ \&-

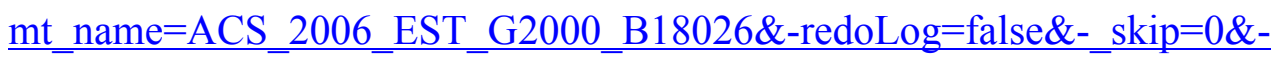

geo_id $=01000 U S \&$-_showChild $=Y \&$-format $=\&$-_lang $=$ en\&-

_toggle=ACS_2006_EST_G2000_B18026 Accessed November 23, 2011.

118.U.S. Census Bureau. United States and States: Percent of People 21 to 64 Years Old

With a Disability: 2007, http://factfinder.census.gov/servlet/GRTTableSS? bm=y\&-

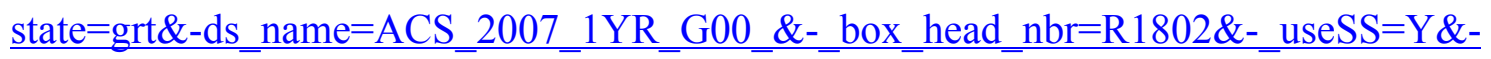
CONTEXT $=$ grt\&-mt_name=ACS_2006_EST_G00_R1802_US30\&-redoLog=false\&geo_id=01000US\&-format=US-30 Accessed November 23, 2011

119.Usability (2013). http://en.wikipedia.org/wiki/Usability 
120.Viterbi, A.J. (1967) Error Bounds for convolutional codes and an optimal asymptotically decoding algorithm. IEEE Translations on Information Theory, IT-13, pages 260-269.

121.VTPlayer (2009). http://vtplayer.sourceforge.net/. Accessed July 7, 2009.

122.W3C Comparison of WCAG 1.0 Checkpoints to WCAG 2.0 (2009)

http://www.w3.org/TR/2006/WD-WCAG20-20060427/appendixD.html Accessed February 7, 2009.

123.W3C Complete List of Web Accessibility Evaluation Tools (2009) http://www.w3.org/WAI/ER/tools/complete Accessed February 7, 2009.

124.W3C (2000) HTML techniques for Web Content Accessibility Guidelines 1.0 http://www.w3.org/TR/WCAG10-HTML-TECHS/, Accessed March 21, 2008. 125.W3C (2006) Roadmap for Accessible Rich Internet Applications. WAI-ARIA Roadmap, December 20， 2006. http://www.w3.org/TR/aria-roadmap, Accessed March 26, 2008.

126.W3C HTML techniques for Web Content Accessibility Guidelines 2.0. http://www.w3.org/TR/WCAG20/ Accessed September 22, 2009.

127.WAI-ARIA Roles (2009). http://www.w3.org/TR/2007/WD-aria-role-20071019/ Accessed September 22, 2009.

128.WAI-ARIA User Agents. (2013). http://www.w3.org/TR/wai-aria-implementation/ 129.Watanabe, M., Okano, A., Asano, Y., Ogawa, K. (2007). VoiceBlog: Universally Designed Voice Browser. International Journal of Human-Computer Interaction, 23(12), pages 95-113. 
130.Watters, P., Araujo, A., Hezart, A., Naik, S. (2005) Accessibility Solutions for Visually Impaired Users of Web Discussion Boards. Proceedings: The Third International Conference on Information Technology and Applications.

131.WCAG2.0@ the Pickards (2009).

http://www.thepickards.co.uk/Articles/WCAG20_Introduction.cfm\#successcrit Accessed August 22, 2009.

132.Web Accessibility Researcher's Blog (2009).

http://webinsight.cs.washington.edu/blog/ Accessed September 22, 2009.

133.Websites of the Internet Relay Chat Community (2007) http://www.irc.com.

134.Winberg, F., Bowers, J. (2004). Assembling the Senses: Towards the Design of Cooperative Interfaces for Visually impaired Users. Proc. CSCW '04, Chicago, IL, USA, Nov. 6-10, 2004, pages 332-341.

135.Woodfine, B. P., Nunes, M. B., and Wright, D. J. (2008). Text-based synchronous elearning and dyslexia: Not necessarily the perfect match! Compute. Educ.50, 3(Apr.2008), 703-717. http://dx.doi.org/10.1016/j.compedu.2006.08.010

136. Word Add-Ins. http://msdn.microsoft.com/enus/library/office/aa189710(v=office.10).aspx. Accessed November 23, 2013. 137. World Health Organization (WHO), http://www.who.int/mediacentre/factsheets/fs282/en/ Accessed November 23, 2013. 138.Xpeers. (2004) April 3, 2008. http://www.xpeers.net 139.Zirzi, R.: (1992) Refining the test phase of usability studies: An empirical evaluation. Proceedings of the ACM Conference on Human Factors in Computer Science, 631-639. 


\section{CURRICULUM VITA}

NAME:

John Gerard Schoeberlein

PERMANENT ADDRESS:

9 Chatterly Court

Perry Hall, Maryland 21128

PROGRAM OF STUDY:

Information Technology

DEGREE AND DATE CONFERRED:

Doctor of Science, 2013 


\section{EDUCATION:}

Towson University - Doctor of Science Degree - Information Technology.

December, 2013, GPA: 4.0.

Upsilon Pi Epsilon - Computer Science National Honor Society.

Omicron Delta Kappa - Leadership National Honor Society.

Dissertation: “Accessible Collaborative Writing for Persons who are blind."

University of Baltimore - Master of Science Degree - Management Information Systems.

December 1993, GPA: 3.893.

Beta Gamma Sigma - Business National Honor Society.

Towson University - Bachelor of Science Degree - Business Administration.

June 1980, GPA: 3.1. 


\section{PROFESSIONAL PUBLICATIONS:}

Schoeberlein, J., Wang, Y.: (2009) Evaluating Groupware Accessibility, In the Proceedings of HCII $200913^{\text {th }}$ International Conference on Human-Computer Interaction, LNCS 5616, pp. 414-423, July 19-24, 2009, San Diego, CA. USA.

Schoeberlein, J., Wang, Y.: (2009) Groupware Accessibility for Persons with Disabilities, In the Proceedings of HCII $200913^{\text {th }}$ International Conference on Human-Computer Interaction, LNCS 5616, pp. 404-413, July 19-24, 2009, San Diego, CA. USA.

Schoeberlein, J., Wang, Y.: (2011) Examining the Current State of Group Support Accessibility: A Focus Group Study, Universal Access In Human-Computer Interaction, In the Proceedings of HCII $201114^{\text {th }}$ International Conference on HumanComputer Interaction, LNCS 6768, pp. 272-281, July 9-14, 2011, Orlando, FL, USA.

Schoeberlein, J., Wang, Y.: (2011) Examining the Current State of Group Support Accessibility: An Expanded Study, In the Proceedings of HCII 2011 14 ${ }^{\text {th }}$ International Conference on Human-Computer Interaction, Communications in Computer and Information Science, LNCS 173, pp. 389 - 393, July 9-14, 2011, Orlando, FL, USA. Schoeberlein, J., Wang, J.: (2012) Accessible Collaborative Writing for the Blind: A Usability Study. In the Proceedings of ASSETS' 2012, 267-268, Boulder, CO, USA. Schoeberlein, J., Wang, Y.: (2013) Providing an Accessible Track Change Feature for Persons Who Are Blind, In the Proceedings of HCII $201315^{\text {th }}$ International Conference on Human-Computer Interaction, UAHCI/HCII 2013, Part III, LNCS 8011, pp. 389-398, July 21-26, 2013, Las Vegas, Nevada, USA. 
Schoeberlein, J., Wang, Y.: (2013) Improving Collaborative Writing for the Blind, Accessibility and Computing, 107, pp. 11-16, September 2013. 


\section{PROFESSIONAL PUBLICATIONS PENDING:}

Schoeberlein, J., Wang, Y.: (2013) Usability Evaluation of an Accessible Collaborative Writing Prototype for Blind Users, Journal of Usability Studies.

\section{COPYRIGHT MATERIAL:}

Schoeberlein, J., Wang, Y.: (2013) CollaborateWithWord and

CollaborateWithWord2007. 
